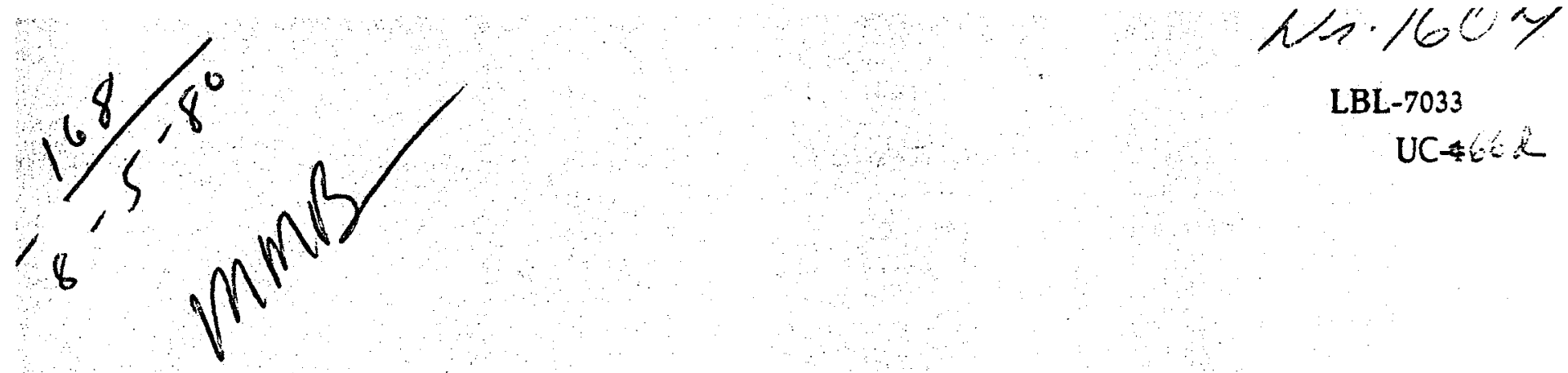

\title{
KINETICS OF SILICA POLYMERIZATION
}

Oleh Weres, Andrew Yee, Leon Tsao

May 1980

Prepared for the U.S. Department of Energy

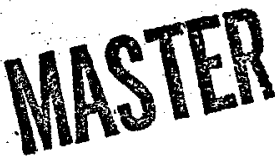
under Contract W-7405-ENG-48

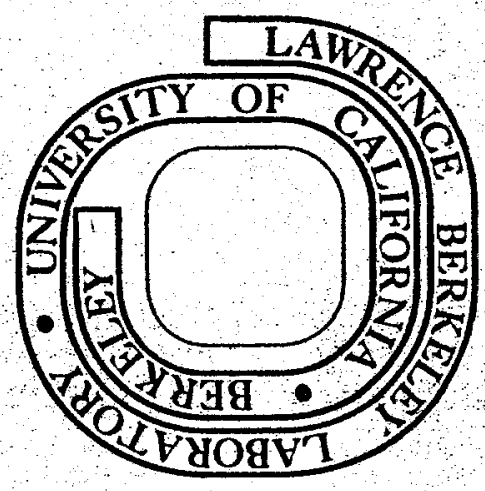




\section{DISCLAIMER}

This report was prepared as an account of work sponsored by an agency of the United States Government. Neither the United States Government nor any agency Thereof, nor any of their employees, makes any warranty, express or implied, or assumes any legal liability or responsibility for the accuracy, completeness, or usefulness of any information, apparatus, product, or process disclosed, or represents that its use would not infringe privately owned rights. Reference herein to any specific commercial product, process, or service by trade name, trademark, manufacturer, or otherwise does not necessarily constitute or imply its endorsement, recommendation, or favoring by the United States Government or any agency thereof. The views and opinions of authors expressed herein do not necessarily state or reflect those of the United States Government or any agency thereof. 


\section{DISCLAIMER}

Portions of this document may be illegible in electronic image products. Images are produced from the best available original document. 


\section{RINETICS OF SILICA POLMERIZATION}

by

Oleh Weres

Andrew Yee

Leon Tsao

Lay 1980

\section{Earth Sciences Division}

Lawrence Berkeley Laboratory

University of California

Berkeley, Callfornla 94720

Prepared for the U.S. Department of Energy under

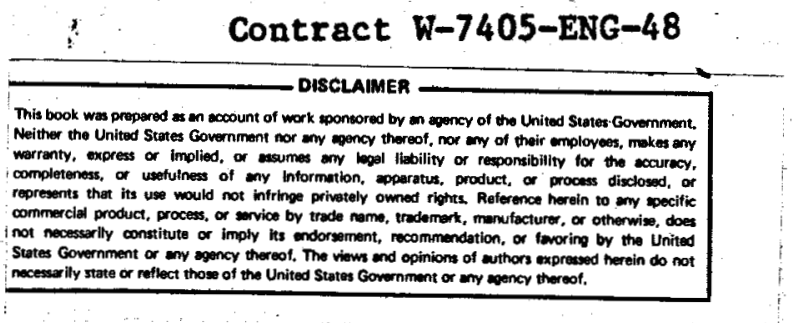


11

When shooting, aim.

- Ukrainian Proverb 
ABSTRACT

The polymerization of silfcic acid in geothermal brine-like aqueous solutions to produce amorphous sllica in colloldal form has been studied experimentally and theoretically. A large amount of high quality experimental data has been generated over the temperature range 23 to $100^{\circ} \mathrm{C}$. Wide ranges of dissolved silica concentration, $\mathrm{pH}$, and sodium chloride concentration were covered. The catalytic effects of fluoride and the reaction inhibiting effects of aluminum and boron were studied also. Two basic processes have been separately studied: the formation of new colloidal particles by the homogeneous nucleation process and the deposition of dissolved silica on pre-existing colloldal particles.

A rigorous theory of the formation of colloldal particles of amorphous silica by homogeneous nucleation was developed. Th1s theory employs the Lothe-Pound formalism, and is embodied in the computer code sILNuc which quantitatively models the homogeneous nucleation and growth of colloidal silica particles in more than enough detall for practical application. The theory and code were extensively used in planning the experimental work and analyzing the data produced. The code is now complete and running in its final form. It is capable of reproducing most of the experimental results to within experimental error. It is also capable of extrapolation to experimentally inaccessible conditions, 1.e., high temperatures, rapldly varylng temperature and $\mathrm{pH}$, etc. Aside from 1ts practical utility, the theoretical work reported here is probably the most extensive and detalled application of homogeneous nucleation theory to a real phystcal system to date.

The literature on aspects of the chemistry of amorphous silica that were not directly studied by us has been extensively reviewed. This review covers both fundamental chemlcal propertles and the practical experience gained working with geothermal brines throughout the world. 

Abstract

Table of Contents

v

List of Tables

vi1

List of Figures

Chapter 1 - Introduction

S1.1 The Scope of this Report

S1.2 Acknowledgements

Chapter 2 - Topics in the Aqueous Chemistry of Amorphous Silica: A Review and Synthesis of the Literature

S2.1 Introduction

S2.2 Solubility of Silica: The Ef fect of Temperature, Salinity and $\mathrm{pH}$ - nornmon

S2.3 Complexing of Dissolved Silica by Ions 12

S2.4 Amorphous Silica and Quartz 15

S2.5 Deposition of S1lica Upon Other Surfaces 17

S2.6. The Surface Structure of Amorphous S111ca 18

S2.7 The Partially Hydrophobic Nature of the Amorphous

S2.8 The Electronic Structure of silicon Dioxide $\quad 21$

S2.9 The Acid-Base Properties of Surface Silanols 23

S2.10 The First Steps of Silica Polymerization: Dimer to
Pentamer

S2.11 Silica Polymerization: Hexamer and Beyond 28

S2.12 The Molecular Mechanism of S1lica Polymerization 29

S2.13 The Base-Catalyzed Condensation Reaction $\quad 32$

S2.14 Catalysis by Hydrogen Fluoride and Hydrogen Ion $\quad 34$

S2.15 Other Catalysts 39

S2.16 Toward Predicting the Rates of Molecular Deposition 39

S2.17 The Nucleation of Colloldal Amorphous Silica 43

S2.18 The "Induction TIme" for Nucleation and the value of the Surface Tension 46

S2.19 The Practical Significance of Nucleation Phenomena and the Study of Homogeneous Nucleation $\quad 49$

S2.20 Other Nucleation Phenomena 51

S2.21 Colloidal Stability and Coagulation of Colloldal Amorphous Silica

S2.22 The Mechanism of Destabilization 57

S2.23 Ion Exchange on the Surface of Amorphous S111ca

S2.24 The Gelation of Collo1dal S111ca 61

Chapter 3 - The Kinetics of Sillca Polymerization In Aqueous Solution

S3.1 Introduct1on $\quad 63$

S3.2 Experimental Methods $\quad 64$

- Timing of the experiments $\quad 65$

- Temperature 
- Silica stock solution

- Buffers

- Colloidal silica

- Other materials and water

- Preparation of the experimental solutions

- Concentration units employed

- Reaction vessels

- $\mathrm{pH}$ Measurement

S3.3 The Rate of Molecular Deposition as a Function of $\mathrm{pH} \quad 72$

- Fitting the data

- Introducing a standard state

- The separate effects of $\mathrm{pH}$ and $\mathrm{pH}_{\mathrm{nom}} \quad 77$

- Sources of error

- Polymerization rates at high $\mathrm{pH} \quad \ldots \quad 80$

S3.4 The Rate of Molecular Deposition $\quad 82$

- Experimental data

- Data reduction

- Trial rate functions

85

- Final results

S3.10 Homogeneous Nucleation: Data Reduction and the Surface Tension

S3.11 Solubility of Silica in Salt Solutions 114

S3.12 The Effect of Added Sodium Chloride 116

S3.13 The Effects of Other Salts 120

S3.14 Methods of Practical Prediction 125

- A Sample Calculation 131

S3.15 Inhibition by Aluminum and Boron 134

A3.1 Empirically Fitted Formulas and Tables for the pH Functions 137

A3.2 Further Experimental Details 141

- The continuous flow kinetic system 141

- Adsorbed silica determination $\quad$. 144

- Spectrophotometric determination of silica 145

- Materials for molybdate analysis 146

- Procedure: molybdate yellow method 146

- Procedure: molybdate blue method 147

- Preparation of a primary silicon standard solution $\quad 147$

- Characterization of colloidal silica sols $\quad 148$

- Surface area determination by the sears titration $\quad 149$ method

A3.3 Sources of Supplementary Data and Further Details of 149

Data Reduction

Chapter 4 - Silica and the Reinjection of Geothermal Brines

S4.1 Introduction

S4.2 Types of Silica Deposits 
S4.3 Possible Mechanism for Post-Reinjection Plugging by Silica

S4.4 Brine Treatment for silica Control $\quad 172$

- Avoldance or minimization of silica supersaturation

- Kinetic Inhibition of molecular deposition and nucleation

- "Aging" the brine to convert dissolved sillca to colloldal sillca

- Removal of colloidal s1lica by coagulation and settling

54.5 Field Experience with Geothermal Relnjection

S4.6 Outstanding Research Needs

Chapter 5 - The Status of Geothermal Brine Treatment Technology

$$
\text { Overview }
$$

S5.1 The Nature of the Problem

S5.2 Brine Treatment Technology

S5.3 Outstanding Research Needs

Chapter 6 - Documentation for Computer Code SILNUC

S6.1

Introduction

195

56.2

The Basic Algorithms in SILNUC

S6.3 Program Structure

56.4

Control of Printed Output

S6.5

Control of Timesteps and the Start of Calculation

204

56.6

Input and Default Values

208

56.7

Limitations and Precautions

212

56.8

A Sample Calculation

214

A6.1

Full Listing of Program SILNUC

220

List of References

231

\section{L1st of Tables}

2.1 Solubility of Amorphous Silica and Quartz In Pure Water 7

2.2 Solubility of Amorphous Silica and Quartz in NaCl Solutions 10

2.3 Complexing Constants of $\mathrm{Mg}^{+2}$ and $\mathrm{Ca}^{+2}$ with Monosilicate Ions at $25^{\circ} \mathrm{C}$ in $1 \mathrm{M} \mathrm{NaClO} 4$

2.4 Catalytic Coefficlents for Varlous Nucleophilic Catalysts of the Silica Depolymerization Reaction

3.1 Fitting of Homogeneous Nucleation Data

3.2 "Reference Values" to be Used with Figures 3.24 to 28

A3.1 Values of $\mathrm{f}^{\prime}(\mathrm{pH})$ vs. $\mathrm{pH}$

A3.3 Formulas and Values for Calculating Activity Coefficients and $\alpha_{s 11}$

A3.4 Molecular Deposition Data for $50^{\circ} \mathrm{C}$ 
A3.5 Molecular Deposition Data for $75^{\circ} \mathrm{C} \quad 157$

A3.6 Molecular Deposition Data for $100^{\circ} \mathrm{C} \quad 158$

A3.7 Fluoride Catalysis Data: Homogeneous Nucleation at $70^{\circ} \mathrm{C} \quad 159$

A3.8 Homogeneous Nucleation Data for $23^{\circ} \mathrm{C} \quad 160$

A3.9 Homogeneous Nucleation Data for $30^{\circ} \mathrm{C} \quad 161$

A3.10 Homogeneous Nucleation Data for $50^{\circ} \mathrm{C} \quad 162$

A3.11 Homogeneous Nucleation Data for $75^{\circ} \mathrm{C} \quad 163$

A3.12 Homogeneous Nucleation Data for $100^{\circ} \mathrm{C} \quad 164$

A3.13 Effect of Sodium Chloride on Homogeneous Nucleation: Selected Data for 30 and $100^{\circ} \mathrm{C}$

A3.14 Effect of Sodium Chloride on Homogeneous Nucleation: Selected Data for $50^{\circ} \mathrm{C}$

A3.15 Effect of Sodium Chloride on Homogeneous Nucleation: Selected Data for $75^{\circ} \mathrm{C}$

4.1 Silica Concentrations, Saturation Ratios, and Deposition Rates in Flashed Geothermal Brines

6.1 Listing of Input Deck for SILNUC Sample Problem 220

6.2 Output from SILNUC Sample Problem

List of Figures

2.1 Known and Suspected Silicic Acid 01igomer Structures. 31

3.1 Continuous Flow Kinetic System for Experiments at $100^{\circ} \mathrm{C}$

3.2 Effect of Varying $\mathrm{pH}$

3.3 Graph of d $\log \mathrm{f}(\mathrm{pH}) / \mathrm{d}$ pH vs $\cdot \mathrm{pH} \quad 75$

3.4 Variation of Deposition Rate with $\mathrm{pH} \quad 78$

3.5 Molecular Deposition at High pH $\quad 81$

3.6 Homogeneous Nucleation at High pH $\quad 81$

3.7 Continuous Flow System Kinetic Data $\quad 83$

3.8 Molecular Deposition Data $\quad 83$

3.9 Residual Scatter of Rate Constant Values $\quad 89$

3.10 Rates of Molecular Deposition at $\mathrm{pH}_{\text {nom }}=\mathrm{pH}=7.0 \quad 90$

3.11 Adsorbed Silica vs. Dissolved Silica Concentration at $50^{\circ} \mathrm{C} \quad 92$

3.12 Fluoride Catalysis Data at $5^{\circ} \mathrm{C} \quad \cdots 5$

3.13 Rate of Fluoride Catalyzed Pathway Alone vs • pH 96

3.14 Homogeneous Nucleation Data for $100^{\circ} \mathrm{C} \quad 105$

3.15 Homogeneous Nucleation Data for $75^{\circ} \mathrm{C} \quad 106$

3.16 Homogeneous Nucleation Data for $50^{\circ} \mathrm{C} \quad 106$

3.17 Homogeneous Nucleation Data for 23 and $30^{\circ} \mathrm{C} \quad 107$

3.18 Comparison of Experimental and Calculated Homogeneous
Nucleation Curves

3.19 Effect of $\mathrm{NaCl}$ on Homogeneous Nucleation $\quad 116$

3.20 Comparison of Experimental and Calculated Homogeneous

3.21 Effect of Varying the Cation $\quad 121$

3.22 Effect of Varying the Anion $\quad 122$

3.23 Effect of Sodiun Sulfate $\quad 123$

3.24 Theoretically Calculated Homogeneous Nucleation Curves
for $50^{\circ} \mathrm{C}$ 
3.25 Theoretically Calculated Homogeneous Nucleation Curves for $75^{\circ} \mathrm{C}$

3.26 Theoret1cally Calculated Homogeneous Nucleation Curves for $100^{\circ} \mathrm{C}$

3.27 Theoretically Calculated Homogeneous Nucleation Curves for $125^{\circ} \mathrm{C}$

3.28 Theoretically Calculated Homogeneous Nucleation Curves for $150^{\circ} \mathrm{C}$

3.29 Effects of Aluminum

135

3.30 Effect of Boron

136

5.1 Generalized Schematic of Avallable Brine Treatment Processes 
CHAPTER ONE - INTRODUCTION

\section{S1.1 The Scope of this Report}

The precipitation of colloldal amorphous silica from brines and the deposition of amorphous silica scale cause serious practical problems in a number of important geothermal resource areas. Unfortunately, it is in precisely those geothermal resource areas whose brines are hot ter and, thereby, most sultable for power generation that the greatest problems with silica scaling and precipitation are encountered. These resource areas Include Niland and the Baca site In the United States; Cerro Prieto and Los Azufres In Mexico, and Walrakel and Broadlands in New Zealand.

Complex chemical processes are involved in the precipitation of amorphous silica from geothermal brines. These Include the deposition of dissolved silica on solid surfaces molecule by molecule ("molecular deposition"), the formation of colloldal amorphous silica particles by homogeneous nucleation, heterogeneous nucleation, the growth of colloldal silica particles by further molecular. deposition of dissolved silica upon them, the flocculation or coagulation of colloidal silica by electrolytes, its adheston to solid surfaces, and the cementation of these deposits to form solld silica scale. That this list Includes unsolved generic problems in physical chemistry suggests the magnitude of the task of properly describing and predicting the precipitation of amorphous sillca from geothermal brines.

Fortunately, in most of the high temperature resource areas the preclpitation of nearly pure colloldal amorphous sillca formed by homogeneous nucleation is the dominant process. Amorphous and crystalline silicate phases are also commonly observed, but are usually present in much smaller amounts. Therefore, the maj or practical questions can be addressed by studying the precipitation of pure colloldal amorphous silica alone.

The goal of the work reported herein was to lay a firm scientific basis for describing and predicting the precipltation of amorphous silica from geothermal brines. The work began with an extensive review of the 11terature on the fundamental chemical processes involved. This review is presented in Chapter 2 of this report. 
The most important processes are the formation of colloldal silica particles by homogeneous nucleation and their further growth by the molecular deposition of dissolved silica upon them. These two process were extensively studied both experimentally and theoretically. These studies are reported in Chapter 3. They were successful, and the results obtained allow meaningful quantitative predictions to be made throughout most of the range of physical conditions of practical interest. This predictive capability is embodied in the computer code SILNUC which is documented in Chapter 6 of this report.

Practical experience gained in working with silica rich geothermal brines in various geothermal fields throughout the world is reviewed in Chapters 4 and 5. Chapter 4 focuses on the Important problem of removal of amorphous silica from geothermal brines prior to relnjection. Chapter 5 discusses the problem of treatment of geothermal brine from a general perspective.

The mechanism of formation of solid silica scale is discussed in Chapter 4. The basic principles and phenomenology of the flocculation and coagulation of colloldal silica are discussed in Chapter 2, and the practical aspects of this subject as they apply to preinjection brines treatment are discussed in Chapter 4. Our original work in these areas is presented in a separate report :(Weres et al., 1980).

This report is an anthology rather than a unitary work. Its various Chapters were written separately and may be read separately. Chapter 4 is excerpted from a longer paper that was presented by 0 . Weres and J.A. Apps at Recent Trends in Hydrogeology - A Symposlum Honoring Paul A. Witherspoon on his Sixtieth Birthday, which was held at the Lawrence Berkeley Laboratory, February 8 and 9, 1979. The proceedings of this Symposium w111 be published In book form in the near future. Chapter 5 was originally presented as a paper at the Electric Power Research Institute's Third Geothermal Conference and Workshop which was held in Monterey, June 26-29, 1979. This conference paper has its own report number, LBL-9249, and w111 soon appear as part of the published proceedings of that conference. Although Chapters 2, 3 and 6 have not been presented or published elsewhere, they differ in form and content, and each may be read almost independently of the others as well. A small amount of redundant material has been allowed to remain in order to preserve the separate readability of the various Chapters. 
Throughout this report, large and small numbers are written in the kind of scientific notation that is used in computer work; e.g., $1.2 \mathrm{E} 6=1.2 \times 10^{6}$.

\section{S1.2 Acknowledgements}

Th1s report was prepared for the United States Department of Energy under Contract W-7405-ENG-48.

The preparation of Chapter 2 , the experimental and theoret1cal work reported In Chapter 3 , and the development of the computer code SILNUC were supported by the Geochemistry and Materlals Research Program of the Division of Geothermal Energy of the US-DOE. The preparation of Chapters, 4 and 5 , part of the analysis of the data reported in Chapter 3 and part of the preparation of Chapter 3 were supported by the Geothermal Reservior EngineerIng Program of DGE through the Cerro Prleto Research Project at LBL.

The following were among those who contributed adv ice, encouragement, and Information to this work: A. J Adduc1, J.A. Apps, L.V. Benson, E. G .Bohlmann, R.H. Busey, C.L. Carnahan, F.W. Dickson, R.C. Feber, R.O. Fournier, R.K. Iler, S.M. Klainer, M.J. Lippmann, A. Manon M., E. Matijevic, R.E. Mesmer, G.A. Parks, M. Reed, H.P. Rothbaum, and A.H. Truesdell.

S. Buchanan helped with some of the computer work reported In Chapter 3 , and prepared some of the Figures.

P. Butler and $T$. Kho typed most of the manuscript.

The authors wish to gratefully acknowledge all of these contributions. 
CHAPTER TWO - TOPICS IN THE AQUEOUS CHEMISTRY OF AMORPHOUS SILICA: A REVIEW AND SYNTHESIS OF THE LITERATURE

\section{S2.1 Introduction}

This Chapter is a review of the iiterature on those basic chemical properties of amorphous sllica which may be relevant to its chemical behavior in geothermal brines and brine-like solutions. This review was executed prior to the start of experimental work and contributed to its planning. Portions of it were later revised in light of the new data. 0ther portions that were rendered obsolete by our own work have been completely dropped. The reader is reassured that the major gaps in this review have been filled by our own work.

It appears that under most conditions silica precipitates as relatively pure amorphous silica. The scope of this review, as well as that of our own work, was restricted accordingly.

The process of amorphous silica precipitation from supersaturated bulk aqueous phase consists of the following steps:

1) Formation of silica polymers of less than critical nucleus size.

2) Nucleation of an amorphous silica phase (from here on simply AS) in the form of colloidal particles.

3) Growth of the supercritical AS particles by further chemical deposition of silicic acid on their surfaces.

4) Coagulation or flocculation of colloidal particles to give either a precipitate or a semisolid material.

5) Cementation of the particles in the deposit by chemical bonding and further deposition of silica between them.

6) Rarely, growth of a secondary phase in the interstices between the AS particles.

When a solid surface is present, a layer of amorphous silica forms on it, and further deposition may proceed as step 3) alone. If an AS colloid is present in the medium, the particles may adhere to the surface in analogy to steps 4) and 5), and step 6) may follow. 
Our original work consisted of an exhaustive quantitative experimental and theoretical study of steps 2) and 3 ). There 1s very 11ttle information of this sort avallable in the 11terature. Therefore, the discussion of these processes in this review is, perforce, of a general background nature and emphasizes the qualitative aspects of these matters.

On the other hand, the extensive and often usable (if only in a semiquantitative sense) information about steps 1) and 4) that is avallable in the 11terature has been reviewed and discussed in detail.

Important subsidiary topics such as the nature of silica surface are also discussed here. Considerable space has been devoted to reviewing important German and Russian language materlals to which the reader might not have access otherwise.

In a few places, our own recent results are also quoted in order to fill in what might otherwise be major gaps in the narrative. In all such cases, the new results referred to are to be found in other Chapters of this report.

Detalled reviews of the practical aspects of the chemistry of silica in geothermal brines and of brine treatment methods are presented in Chapters IV and $V$ of this report.

This review is strong (even definitive) in some areas, but by no means exhaustive. The serious reader is referred to R.K. Iler's (1979) massive recent book for a much more extensive review of the general field of silica chemistry. There is much material in Iler's book that should, by rights, also have been in this review. However, because this review was in an advanced draft stage when the book appeared, 11 ttle attempt was made to assimilate material from the book.

\section{S2.2 Solubility of Silica: The Effect of Temperature, Salinity and $\mathrm{pH}$}

Silica in geothermal brines may come from a variety of sources. Its concentration is usually controlled by the solubility of quartz. The precipitation of AS is controlled by AS solubility.

The solubilities of $A S$ and quartz in pure water at various temperatures are presented in Table 2.1. Up to $250^{\circ} \mathrm{C}$ and at saturation pressure, the equilibrium solubility of $\mathrm{AS} 1 \mathrm{n} \mathrm{g} / \mathrm{kg}$ of water $1 \mathrm{~s}$ given by the equation: 
$\log c_{0}=-731 / \mathrm{T}+1.52$

(Fournier and Rowe, 1977)

Up to about $220^{\circ} \mathrm{C}$, the solubility of quartz is given by the equation

$$
\log c_{0}=-1160 / T+1.93
$$

(An empirical fit to data collected in an unpublished literature search by S. Cosner and J.A. Apps of this Laboratory.)

At higher temperatures the solubility of both forms drops below these curves because of the decreasing density and solvent power of water. The solubility reaches a maximum at about $340^{\circ} \mathrm{C}$ and then rapidly decreases as the critical point of water is approached.

The fact that quartz solubility controls initial silica concentration in brines while AS precipitates greatly reduces the incidence and rate of precipitation. For example, a low salinity brine saturated with quartz at $220^{\circ} \mathrm{C}$ does not become supersaturated with AS until the temperature drops to below about $100^{\circ} \mathrm{C}$. Between $100^{\circ} \mathrm{C}$ and $220^{\circ} \mathrm{C}$ the only silica precipitation to be expected is a slow and very likely kinetically insignificant growth of quartz on some surfaces. Even below $100^{\circ} \mathrm{C}$ the precipitation of silica will be limited to slow and, again, probably insignificant molecular deposition of AS on solid surfaces.

However, if the temperature is low enough and, thereby, the degree of supersaturation great enough, homogeneous nucleation will occur. Available evidence (Harvey et al., 1976; Midkiff and Foyt, 1976, 1977) suggests that a saturation ratio of about two is required for homogeneous nucleation to occur. In our example, this value will be reached when the brine is cooled to about $50^{\circ} \mathrm{C}$. However, at temperatures this low the rate of precipitation is slow and this will delay precipitation although certainly not prevent it. In general, we anticipate that silica precipitation will be a significant problem only with brines of initial temperature above about $200^{\circ} \mathrm{C}$. This seems to be borne out by available field experience. 
Table 2.1

Solubility of Amorphous Sillca and Quartz In Pure Water.

$\left(\mathrm{mg} \mathrm{SIO}_{2} / \mathrm{kg} \mathrm{H} \mathrm{H}_{2} \mathrm{O}\right)$

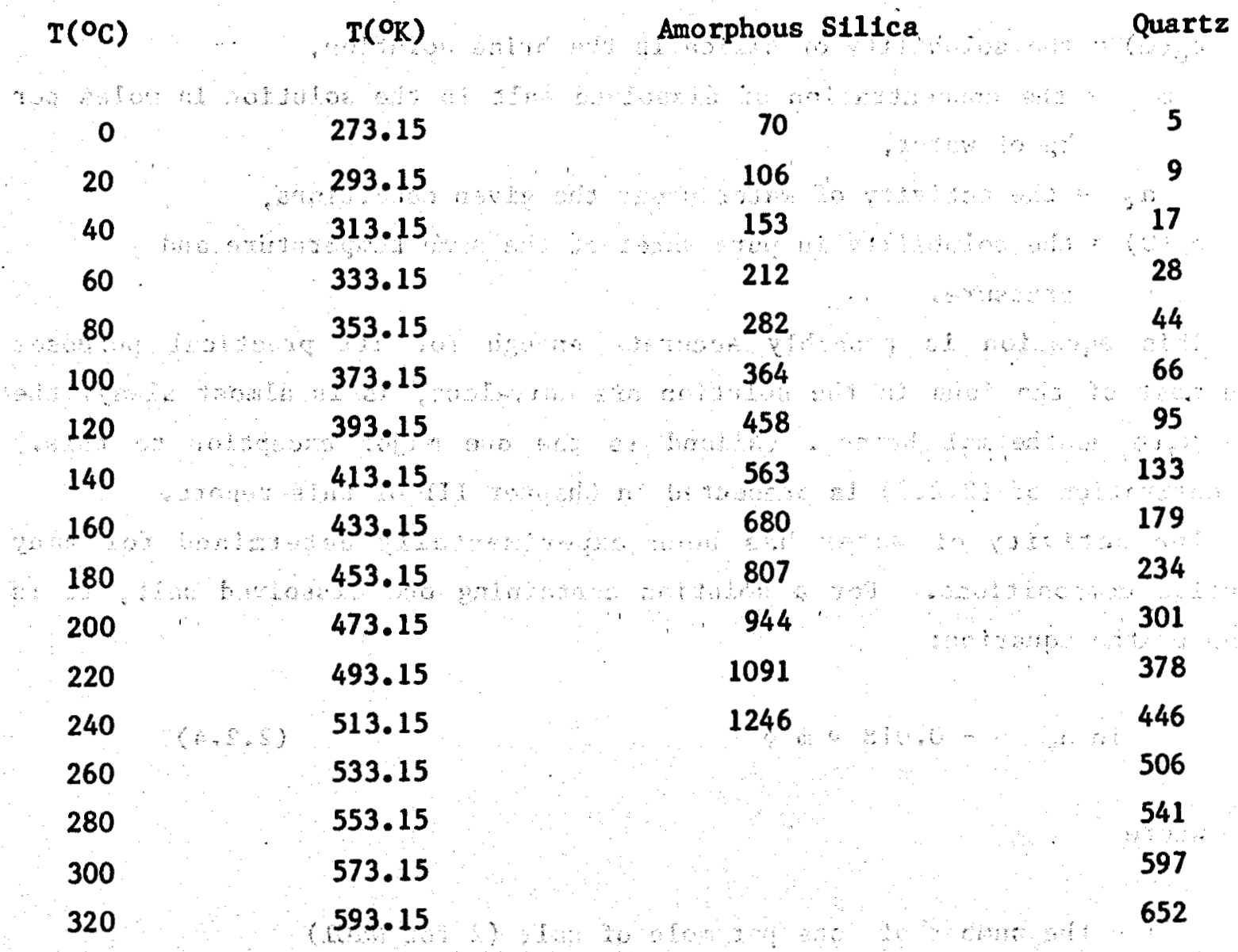

Amorphous silica values from Fournier and Rowe (1977)

Quartz values are a "best fit" to the values of Van Lier, DeBruyn and overbeek (1960), S1ever (1962) and Crerar and Anderson (1971). This f1t was generated by S. Cosner and J.A. Apps (unpublished). 
The salts in the brine also effect the solubility of silica. Using theoretical methods, we have demonstrated that this effect is approximately described by the equation

$$
c_{0}(m)=c_{0}(0) a_{w}
$$

where

$c_{o}(m)=$ the solubility of silica in the brine solution,

m = the concentration of dissolved salt in the solution in moles per $\mathrm{kg}$ of water,

$a_{\mathrm{w}}=$ the activity of water under the given conditions,

$c_{0}(0)=$ the solubility in pure water at the same temperature and pressure.

This equation is probably accurate enough for all practical purposes when most of the ions in the solution are univalent, as is almost always the case with geothermal brines. (Niland is the one major exception to this.) The derivation of $(2.2 .3)$ is presented in Chapter III of this report.

The activity of water has been experimentally determined for many solution compositions. For a solution containing one dissolved salt, it is given by the equation:

$$
\ln a_{w}=-0.018 . \nu \mathrm{m} \phi
$$

where

$$
\begin{aligned}
& v=\text { the number of lons per mole of salt ( } 2 \text { for } \mathrm{NaCl} \text { ) } \\
& \phi=\text { the practical osmotic coefficient }
\end{aligned}
$$

In the case of sodium and potassium chloride solutions, the osmotic coefficient is between 0.9 and 1.0 over a large range of temperature and concentration. 
The solubilities of quartz at $300^{\circ} \mathrm{C}$ and of quartz and As at $100^{\circ} \mathrm{C}$ in $\mathrm{NaCl}$ solutions of various concentrations are presented in Table 2.2 . The effect of the sait is seen to be minor at concentrations up to 1 molal, but is clearly important at 6 molal. The effect is smaller at the higher temperature than at the lower. This means that the saturation ratio will increase faster with falling temperature in brine than in pure water, and this increases the driving force for precipitation in the cooled brine.

$\mathrm{pH}$ has a major effect on silica solubility in the alkaline range. This effect is due to the formation of orthosilicate lon, which constitutes a second species that silica may form when going into solution. The dissociation reaction is:

$$
\mathrm{Si}(\mathrm{OH})_{4}+\mathrm{OH}^{-} \longrightarrow \mathrm{S} 1 \mathrm{O}(\mathrm{OH})_{3}^{-}+\mathrm{H}_{2} \mathrm{O}
$$

Busey and Mesmer (1977) give the following expression for the corresponding concentration product in $\mathrm{NaCl}$ solutions of ionic strength up to 5 :

$$
\begin{aligned}
& \log Q_{11}=2346.69+2.579791 \mathrm{nT}-18.4014 \\
& +0.0964146 \mathrm{I}-3.02800 \mathrm{E}-7 \mathrm{IT}^{2}+0.529703 \mathrm{~F}(\mathrm{I}) \mathrm{I} \\
& +0.0157 \phi \mathrm{I}
\end{aligned}
$$

where

$$
\begin{aligned}
& I=1 \text { onic stength (same as molality for NaCl) } \\
& F(I)=\left[1-\left(1+2 I^{1 / 2}-2 I\right) \exp \left(-2 I^{1 / 2}\right)\right] /(4 I)
\end{aligned}
$$


Solubility of Amorphous \$1lica and Quartz in NaCl Solutions

$$
T=100^{\circ} \mathrm{C}
$$

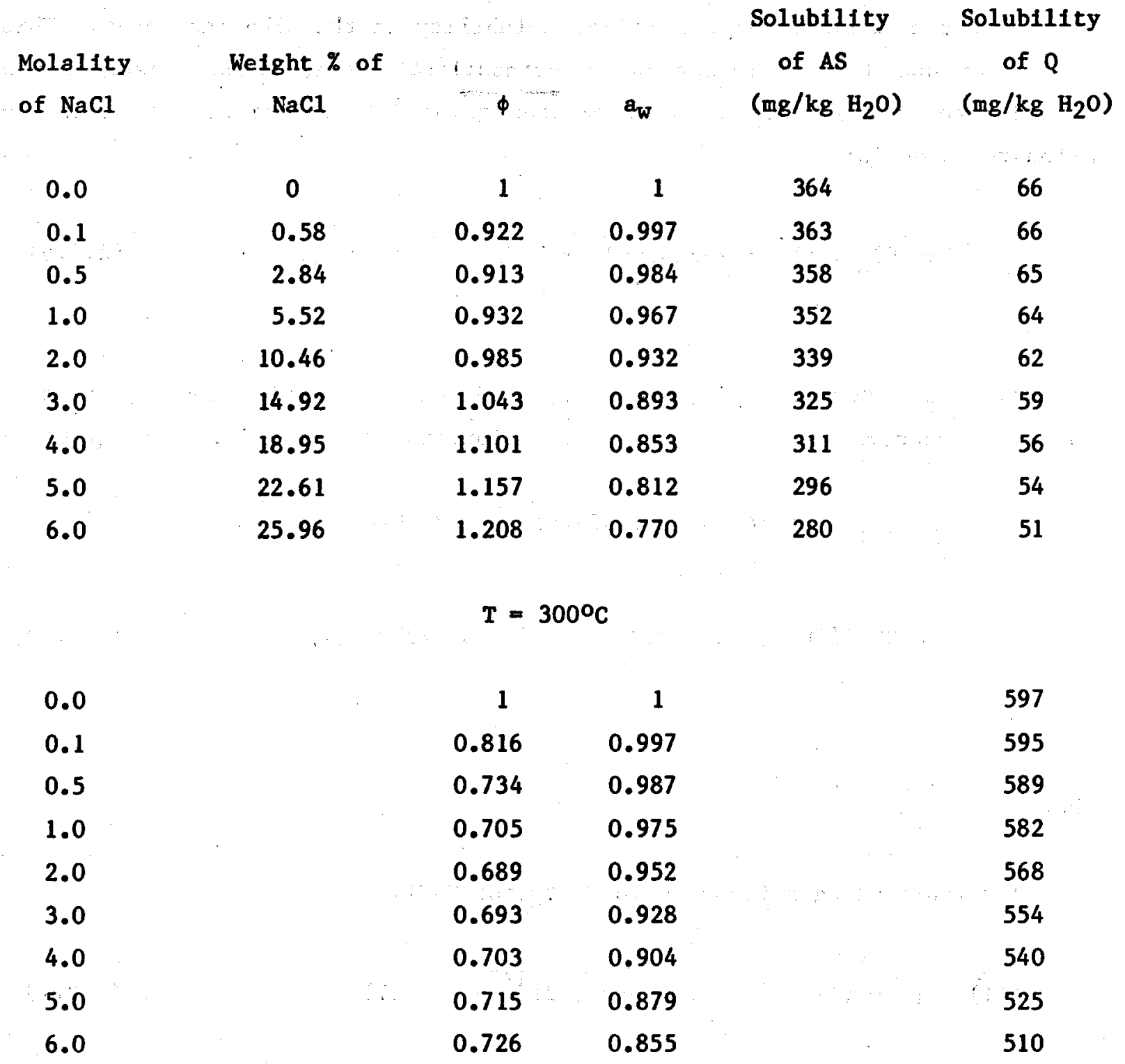

Values of $\phi$ from Sylvester and Pitzer (1976). 
The function $F$ (I) arises from Pitzer's (1973) theory of electrolyte solutions. In (2.2.6), the value of the logarithm of $Q_{11}$ varies strongly with temperature, but only moderately with lonic strength. This equilibrium constant may be converted to that for the equivalent reaction

$$
\mathrm{Si}(\mathrm{OH})_{4} \longrightarrow \mathrm{SiO}(\mathrm{OH})_{3}^{-}+\mathrm{H}^{+}
$$

by combining it with the value of the water ion concentration product under the same conditions. Th1s may be calculated for NaCl solutions of up to 3 molal concentration over the temperature range 0 to $300^{\circ} \mathrm{C}$ using similar formulas given by Busey and Mesmer (1976) in another paper. They also give formulas for $\mathrm{KCl}$ solutions. Here we quote only their formula for the dissoclation equilibrium constant of water in NaCl solutions:

$$
\begin{aligned}
\log \mathrm{K}_{\mathrm{W}}= & 31286 / \mathrm{T}+94.9734 \ln \mathrm{T}-0.097611 \mathrm{~T}-2170870 \mathrm{~T}^{-2} \\
& -606.522
\end{aligned}
$$

For example, at $25^{\circ} \mathrm{C}$ and $\mathrm{I}=0.0$, the $\mathrm{pK}_{\mathrm{a}}$ of MSA $1 \mathrm{~s} 9.82$. Thus, at $\mathrm{pH}=8.50$ the solubility of MSA under these conditions would be about $5 \%$ greater than at acidic $\mathrm{pH}^{\prime} \mathrm{s}$. We conclude that this effect is of small quantitative significance under typical brine conditions as long as the $\mathrm{pH}$ is not above about 8 , but can become extremely Important at higher pH values. (Note also that the $\mathrm{pK}_{\mathrm{a}}$ of MSA decreases with Increasing temperature.)

MSA may release more than one proton to give anions of charge greater than one. The second Ionlzation step is:

$$
\mathrm{SiO}(\mathrm{OH})_{3}^{-} \rightarrow \mathrm{H}^{+}+\mathrm{SHO}_{2}(\mathrm{OH})_{2}^{2-}
$$

Busey and Mesmer (1977) report the $\mathrm{pK}_{\mathrm{a}}$ for this dissociation in 1 molal $\mathrm{NaCl}$ to be 12.32 at $60^{\circ} \mathrm{C}$ and 10.2 at $300^{\circ} \mathrm{C}$. In comparison, the values of $\mathrm{pk}_{\mathrm{a}}$ for the first dissociation step under the same conditions are about 8.9 
and 7.8 , respectively. It is clear that we may ignore the contribution which the second dissociation step makes to the solubility of silica in geothermal brines.

\section{S2.3 Complexing of Dissolved Silica by Ions}

Dissolved silica is known to react with fluoride solutions to form the silicon hexafluoride ion:

$$
\mathrm{Si}(\mathrm{OH})_{4}+6 \mathrm{~F}^{-}+4 \mathrm{H}^{+} \rightarrow \mathrm{SiF}_{6}^{2-}+4 \mathrm{H}_{2} \mathrm{O}
$$

The equilibrium constant for this reaction is on the order of $1 E 30$ at room temperature and moderate salinity ( $R$. Busey, private communication). Typical concentrations of total F in geothermal brines are 5E-5 to $1 \mathrm{E}-3$ molal. It is easy to determine that this complexing reaction will have only negligible effects upon free $F^{-}$and silica concentrations under the conditions of interest.

Complexes with divalent cations may also be important. Santschi and Schindler (1974) studied the formation of magnesium and calcium complexes:

$$
\begin{array}{lc}
\mathrm{M}^{2+}+\mathrm{H}_{3} \mathrm{SiO}_{4}^{-} \rightarrow \mathrm{M}\left(\mathrm{H}_{3} \mathrm{SiO}_{4}\right)^{+} & \mathrm{K}_{1} \\
\mathrm{M}^{2+}+2 \mathrm{H}_{3} \mathrm{SiO}_{4}^{-} \longrightarrow \mathrm{M}\left(\mathrm{H}_{3} \mathrm{SiO}_{4}\right)_{2} & \mathrm{~K}_{2} \\
\mathrm{M}^{2+}+\mathrm{H}_{2} \mathrm{SiO}_{4}^{2-} \rightarrow \mathrm{M}\left(\mathrm{H}_{2} \mathrm{SiO}_{4}\right) & \mathrm{K}_{2}
\end{array}
$$

where

$$
\mathrm{M}^{2+}=\text { either } \mathrm{Mg}^{2+} \text { or } \mathrm{Ca}^{2+}
$$


Table 2.3 contains the results they obtained. It is easy to determine that these reactions will be important only at the very highest calcium and magnesium concentrations encountered in geothermal brines: At INIland, calclum has been reported at concentrations up to 1 molal, but is much lower elsewhere (typlcally 1E-3 or 1E-2 molal). Magnesium 1s nowhere higher than about 0.1 molal, and is usually much lower. We conclude that complexing by magnesium is insignificant compared to complexing by calcium.

With 1 molal $\mathrm{Ca}^{2+}$, the effect of the first complexing reaction will be to approximately triple the total ampunt of orthosilicate ion in solution. Unmodified Niland brines - both flashed and unflashed - typically have a $\mathrm{pH}$ between 5 and 7 at room temperature." Thus, the concentration of both free and calcium complexed silicate antons in them is negligibly small. Flashed brine in other areas - notably East Mesa and Cerro Prieto - may have a pH as high as 8.5 , but these brines contain far too little calcium and magnesium for quantitatively significant complexing to occur.

Niland brines also contain up to $6 \mathrm{E}-2$ molal divalent 1 ron and manganese, and up to 2E-2 molal zinc. No cillicate complexing data seem to be avallable for these Ions. However, Schindler et al. (1975) found that there is a good linear correlation between the logarithms of the lon exchange coeficients of several divalent lons on the AS surface and the logarithms of their hydroxide complexing coefficients. As a first guess, we may assume that such a Iinear relationship holds in this case also. Combining the data in Table 2.3 with the necessary $\mathrm{OH}^{-}$complexing constants from the compilation of sillen and Martell (1964) allows us to estimate the needed values. For Iron we estimate $\log k_{1}=1.4$ and for manganese, $\log k_{1}=1.1$.

The value for zinc is less certain but is probably roughly the same. If these lons were present in free form in Niland brine, they would have about the same small effect as calcium on sillca solubility. However, they are probably strongly complexed by sulfide and chloride and largely unavallable for complexing with silica. We conclude that complexing by divalent transition metal fons has no effect on sillca solubility even at Niland. 
Table 2.3

Complexing Constants of $\mathrm{Mg}^{2+}$ and $\mathrm{Ca}^{2+}$ with Monosilicate Ions at $25^{\circ} \mathrm{C}$ in $1 \mathrm{M} \mathrm{NaClO} 4$

(From Santschi and Schindler, 1974)

$$
\begin{aligned}
& \mathrm{Mg}^{2+} \quad \mathrm{Ca}^{2+} \\
& \log K_{1} \quad 0.64 \pm 0.06 \quad 0.39 \pm 0.03 \\
& \log K_{2} \cdot 3.82 \pm 0.08 \quad 2.89 \pm 0.07 \\
& \begin{array}{lll}
\log \mathrm{K}_{3} \quad 4.17 \quad 3.09
\end{array} \\
& \mathrm{~K}_{1}=\left[\mathrm{MH}_{3} \mathrm{SHO}_{4}^{+}\right] /\left[\mathrm{M}^{2+}\right]\left[\mathrm{H}_{3} \mathrm{SiO}_{4}^{-}\right]
\end{aligned}
$$

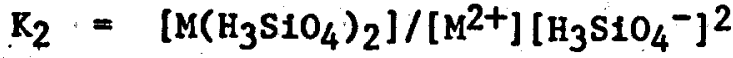

$$
\begin{aligned}
& \mathrm{K}_{3}=\left[\mathrm{M} \mathrm{H}_{2} \mathrm{SiO}_{4}\right] /\left[\mathrm{M}^{2+}\right]\left[\mathrm{H}_{2} \mathrm{SiO}_{4}{ }^{2-}\right]
\end{aligned}
$$


Reardon (1979) has studied the complexation of silica by ferric iron at low $\mathrm{pH}^{\prime} \mathrm{s}$ and has shown $1 \mathrm{t}$ to be Important below about $\mathrm{pH} 2.5$. However, at higher $\mathrm{pH}^{\prime} \mathrm{s}$ the maximum concentration of ferric 1on in solution is markedly. reduced due to the decreasing solubility of Its hydroxide, and Its effect on the solubility of silica becomes unimportant. We conclude that the complexa-; tion of silica by ferric 1ron is unlikely to be practically important in geothermal brines.

Complexing with monovalent lons is probably very weak, and any quantitative effect that it may have is accounted for as part of the effect of salinity on the dissociation constant. The concentration of free trivalent Ions is almost always negligibly small because of the formation of hydroxide and other complexes.

We conclude that complexing with other brine constituents has essentially no effect upon silica solubility. In the case of low and moderate salinity brines this is certain. The paucity of data applicable to Niland brines and their high salinity precludes certainty in this case, but the probability remains high.

For all practical purposes, we can say that the solubility of silica, in geothermal brines 16 controlled mostly by temperature and osmotic pressure, while $\mathrm{pH}$ has a secondary effect.

\section{S2.4 Amorphous Silica and Quartz}

Quartz (as well as tridymite and cristobalite) is thermodynamically more stable than AS. Nonetheless, growth of quartz from aqueous solution has been accomplished at measurable rates only under conditions of extreme temperature and pressure and/or alkalinity. These are precisely the conditions for extremely rapid condensation and dissolution of silica. Thus, the molecules condensing on the surface of a quartz crystal have ample opportunity to rearrange themselves into the thermodynamically optimal quartz structure before they are buried under further layers of molecules.

Under the much milder conditions typlcal of geothermal brines and most laboratory work, the rearrangement of surface molecules is much more sluggish. 
Consider a (partial) layer of monosilicic acid (MSA) molecules condensed onto the surface of a quartz crystal. If this is the first layer deposited on the bulk quartz, its structure will strongly resemble that of quartz, but will also contain some "mistakes". If the time scale for the deposition of additional molecules from solution is comparable to that for rearrangement to near perfect quartz structure or shorter, these errors will be "frozen in" by being covered over by additional layers of freshly condensed silica. The first layer's errors w11l be propagated, and new ones added as more layers are deposited. If the precipitating medium is supersaturated with AS, the ultimate result will be an AS surface which advances by the deposition of more AS upon it from solution.

Such a transition from bulk quartz to amorphous silica has been demonstrated In the laboratory by Baumann (1970). He found that about ten monolayers are required to complete the transition. If the medium is supersaturated with quartz but not with AS, the deposition of silica stops at fewer than ten layers. The final layer deposited under such conditions has a solubility equal to the concentration of MSA in the medium. Its structure is probably intermediate between those of AS and quartz.

Quartz does, of course, form in nature, and the formation of quartz under ambient laboratory conditions has also been reported (Mackenzie and Gees, 1971). Baumann (1970) has suggested that the deposition of quartz from aqueous solution Involves an imperfect surface layer. The rate determining step is probably the conversion of the surface layer structure to the quartz structure, and this rate is probably controlled by the rate of diffusion of structural defects out of the surface layer.

There are a few reports of quartz deposition from geothermal brines and brine-like solutions. Rothbaum and Rohde (1978) studied the polymerization of sllica in very low salinity media at temperatures up to $180^{\circ} \mathrm{C}$. They found that at temperatures up to $100^{\circ} \mathrm{C}$ the dissolved silica concentration would fall to slightly above the equilibrium solubility of amorphous silica and stay there. At higher temperatures, it would first fall to that level and then, and after much longer aging, would fall to slightly above the equilibrium solubility of quartz. However, quartz was not detected by X-ray diffraction in the precipitate that was formed. No indication of quartz formation was observed at lower temperatures. 
Howard et al. (1978) have reported the formation of minor amounts of quartz rich scale at $120^{\circ} \mathrm{C}$ in an experimental desalination unit fed with geothermal brine at East Mesa. Trace amounts of quartz were also reported at temperatures down to $100^{\circ} \mathrm{C}$, but these occurences may well have been due to the incorporation of brine and alrborne rock dust into the scale.

Rimstidt (1979) has reported some data that suggest the deposition of quartz or some other crystalline silica form from nominally salt-free solutions at temperatures as low as 65 and $105^{\circ} \mathrm{C}$.

The practical implication of all this is that a quartz surface will quickly be converted to an AS surface if it is exposed to a solution which is supersaturated with AS. The deposition of sllica from a medium which is undersaturated with AS onto quartz will stop or slow down greatly after only a minute amount has been deposited, even though the medium may be supersaturated with quartz. Therefore, AS is almost always the dominant precipitate under practical conditions, and the degree of supersaturation whth AS plays the chemically dominant role.

\section{S2.5 Deposition of Silica upon other Surfaces}

Very 1ikely, the deposition of silica upon the surfaces of the other silica minerals is analogous to the case with quartz.

The deposition of silica upon other kinds of mineral surfaces has not been studied to date. However, it is unlikely that pure silica precipitates upon them as anything but AS, and even then only from solutions which are supersaturated with AS.

It is belleved that the surfaces of non-noble metals are covered by hydroxyl groups as are the surfaces of the various forms of silica. It is likely that silicic acid can eastly bond to such surfaces. If the medium is supersaturated with AS, an AS surface layer will form and grow. It is unlikely that such growth requires heterogenous nucleation for initiation. If it does, the free energy barrier for nucleation is probably small.

All In all, we conclude that the deposition of silica upon most or all surfaces proceeds as though they were AS surfaces and produces AS. This leads 
to a substantial simplification of our task, because it means that only the kinetics of deposition on an AS surface need be considered.

\section{S2.6 The Surface Structure of Amorphous S111ca}

The bulk structure of AS little resembles that of the varlous crystalline forms, and available evidence suggests that the surface of AS does not resemble that of any of the crystalline forms.

The definitive work in this regard appears to be that of M. M. Egorow and his coworkers at Moscow State University. (For a comprehensive German language revlew, see Egorow et al., 1966.)

The most direct information about the surface structure is that ylelded by Kvlividze's (1964) NMR study of the surface silanolic protons of silica gel dried in vacuum at $200^{\circ} \mathrm{C}$. He was able to fit the proton signal with three Gaussian distributions, each of which he assigned to a different type of 61lanol group.

The narrowest peak was assigned to Isolated silanols whose nearest nelghbour protons lie about 5.2 to 5.4 away. Clearly, such an 1solated silanol group must be the only one attached to its silicon atom, 1.e., a "tertiary silano1". About $25 \%$ of the silanols fall in this group.

A second peak was assigned to pairs of silanols whose protons lie 2.52 to 2.6 $\AA$ apart. It was not possible to determine whether these silanols belong to a single "secondary" silica group, or to two adjacent "tertiary" silica groups. About $30 \%$ of the silanols which were found to fall in this group.

The third and broadest peak was assigned to silanols which have two to four neighbours at a distance of 2.52 to $2.6 \AA$. About $45 \%$ of the silanols were found to belong to this group.

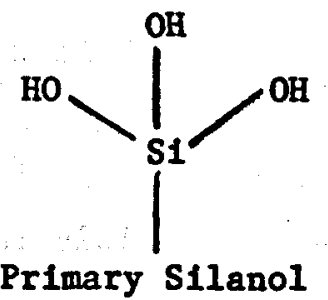

Groups

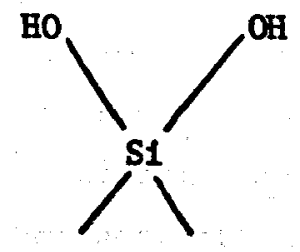

Secondary Stlanol Groups

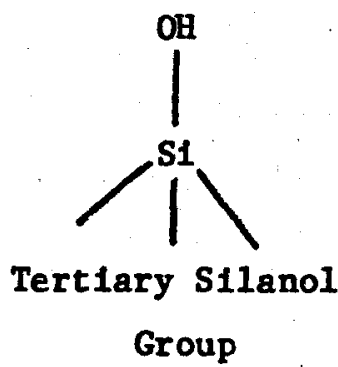

$(2.6 .1)$ 
Most intriguing is the presence of the isolated silanols. The presence of such isolated silanols demands the presence of fairly substantial silanolfree areas around them. A similar argumenent applies to the 1solated pairs of silanols also.

These considerations suggest that the sllica surface is markedly heterogeneous. Apparently, there are surficial patches that contain several closely spaced silanols interspersed with areas in which there are only isolated silanols and pairs of stlanols. Egorow et al. (1966) refer to these two types of domains as stlanolic reglons and slloxanic reglons, respectively. These authors report the water content of thefr dehydrated silica specimens to correspond to 4 to $5 \mu \mathrm{M} \mathrm{H} \mathrm{H}^{-2}$ of surface area (1.e.5 $4.8 \mathrm{E} 14$ to $6.0 \mathrm{E} 14$ oH $\mathrm{cm}^{-2}$ ). These values 11e toward the $10 \mathrm{w}$ end of the range of silanol densities calculated for the varlous crystal faces of tridymite and cristobalite, which again supports the concept of a partlally dehydrated surface.

Egorow and his coworkers used silica dried at $100^{\circ} \mathrm{C}$. It $1 \mathrm{~s}$ possible that this treatment has created a surface less hydrated than that of AS in contact with liquid water. However, this seems unlikely. First, it was found that the drying at $200^{\circ} \mathrm{C}$ produced a surface whose properties were only slightly different from that produced by drying at $50^{\circ} \mathrm{C}$. In particular, only a few percent more water was removed by dehydration at the higher temperatures (Ibid.).

Working with colloldal silica prepared by thermal decomposition of $\mathrm{SICl}_{4}$, Young (1958) found that chemically bound water was removed only by drying above about $170^{\circ} \mathrm{C}$. The extent of surface dehydration at $200^{\circ} \mathrm{C}$ was apparently only a few percent. Thls does not seem to be enough to cause a qualitative change in surface properties.

There appears to be no good reason to reject out of hand the idea that silanol-free areas may exist on an AS surface formed under water and in contact with water. It is generally recognized that there are only very few silanol groups within the bulk structure of AS. The reason is that the silica groups on the surface arrange and rearrange themselves so as to form slloxane bridges (S1-0-S1) with as many nelghbors as possible. This is possible despite the irregularity of the AS structure because the presence 
of the oxygen atom between the two silicons provides great flexibility to the slloxane bridges. Because the bonding through the oxygen is noncolinear, the oxygen serves as a swivel point; also, Si-0-Si bending appears to be easy. Mozzi and Warren (1969) report that the S1-0-S1 bond angle In bulk AS varies between 120 and $180^{\circ}$, with a peak in the distribution at $144^{\circ}$. The surface stlanol groups w11l probably also react and interlink as much as steric factors allow. There is no reason to doubt that this condensation may go so far as to produce a locally silanol-free surface in contact with liquid water. It 1s true that the replacement of wettable silanolic surface with non-wettable slloxanic surface will decrease the stabilization of the surface due to hydration, but this is probably a relatively small effect that is overwhelmed by the chemical driving force for condensation.

The low ion exchange capacity of $3.9 \mathrm{E} 14 \mathrm{~cm}^{-2}$ which may be inferred from the results of Allen, et al. (1977) is also consistent with a more or less maximally condensed and dehydrated AS surface structure.

Finally, this general concept of the appearance of the AS surface is supported by model bullding experiments. Two different "kinetic laws" have been employed to construct models of particles consisting of over fifty monomer units, (A realistic kit with 1410 S1-0-S1 angles was employed.) In both cases irregular particles with highly inhomogeneous surface structures resulted. Distinct and obviously different silanol and siloxane dominated regions of several $\AA$ extent were evident on the surfaces of both models.

\section{S2.7 The Partially Hydrophobic Nature of the Amorphous Silica Surface}

Young (1958) studied water absorption on AS specimens dried at various temperatures. He found that higher drying temperatures caused greater 1rreversible water loss and a greater loss of ability to physically adsorb water vapor. He correlated this decrease in water sorption ability with the loss of surface silanol groups through dehydration, and concluded that water molecules are sorbed only by silanol groups by hydrogen bonding. The siloxanic areas apparently did not sorb water at all, and as their extent increased with a greater degree of Irreversible dehydration, the water sorption capacity of the surface decreased. 
Egorov et al. (1959) studied the heat of water wetting of partially thermally dehydrated silica gels. They found that it slowly increased up to $300^{\circ} \mathrm{C}$ drying temperature, and then rapidly dropped off with higher drying temperature. This maximum correlated well with a pronounced break in the curve of water content versus drying temperature. The rate of increase of dehydration with increasing temperature was observed to sharply increase at about $300^{\circ} \mathrm{C}$. They compared the heats of wetting of different specimens of silica gel dried at $300^{\circ} \mathrm{C}$. The amount of water remaining in each specimen after drying to $300^{\circ} \mathrm{C}$ was somewhat different. An excellent linear relationship was found to hold between the amount of water remaining after drying at $300^{\circ} \mathrm{C}$ and the heat of wetting. Extrapolating the line to zero water content gave an estimate of 50 ergs $\mathrm{cm}^{-2}$ for the heat of wetting of a purely siloxanic surface. Extrapolation to a hypothetical "fully hydrated" surface with $7.0 \mathrm{E} 14 \mathrm{OH} \mathrm{cm}^{-2}$ gave a value of 250 ergs $\mathrm{cm}^{-2}$ for the heat of wetting of such a surface. The lower figure is roughly consistent with a "wetting" Interaction through relatively feeble dispersion and Induced dipole forces. The second value is in perfect agreement with a "wetting model" which involves the formation of one hydrogen bond between each silanol on the surface and an adjacent water molecule.

\section{S2.8 The Electronic Structure of Silicon Dioxide}

Silicon lies directly below carbon in the periodic table. The chemical properties and structural proclivities of the two elements are somewhat similar. Silicon is tetrahedrally coordinated in nearly all instances, and nucleophilic substitution with steric Inversion is a common reaction mechanism.

There are also important differences. One 18 that silicon never engages In $\pi$ bonding which involves its $p$ orbitals. This means that double bonds of the sort carbon, nitrogen and oxygen commonly engage in are impossible for silicon. (Silicon dioxide would probably be a gas if this were not sol)

Another difference is that sllicon has five vacant $3 \mathrm{~d}$ orbitals avallable for dative bonding interactlons which are somewhat similar to ordinary bonding. This has an important Influence upon the properties of $\mathrm{SiO}_{2}$. The underlying phenomenon is the formation of weak partial bonds by the partial entry of free electron pairs of oxygen into the silicon $3 \mathrm{~d}$ orbitals. The effect of this is a transfer of negative charge from oxygen to silicon. 
The canonical structures involved are:

$$
\begin{aligned}
& \geqslant s i-\ddot{0}-s i \leq
\end{aligned}
$$

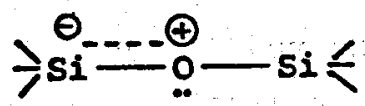

$$
\begin{aligned}
& >s i-\oplus_{0}^{\oplus}-\ldots \stackrel{\Theta}{s i} \leq
\end{aligned}
$$$$
(2.8 .1)
$$

The first "singly bonded" structure is the major one. The other two Involve dative bonding. Each silicon atom can accept electrons from any of its neighboring oxygen atoms in this way.

In general, highly electronegative oxygen pulls some negative charge toward 1t when bonded to a less electronegative element like silicon. This "normal" Inductive effect involves the introduction of some excess electron density into the higher vacant orbitals of the oxygen atom. However, the juxtaposition of free electron pairs on the oxygen atom with the vacant $3 \mathrm{~d}$ orbitals of the silicon is optimal for induction to occur in the opposite direction as described above. The two phenomena apparently just cancel each other out, and the result is a very nearly non-polar S1-0 bond. This lack of polarity and the diminished free electron pair charge density are the cause of the hydrophoblc nature of the slloxanic reglons of the AS surface. The hydrogen bonding inability of slloxanic oxygen in small organosiloxanes has been demonstrated by NMR methods by Huggins (1961) who interpreted his results in approximately the above terms.

Dative bonding is also reflected in the S1-0-Si bond angles of about about $141^{\circ}$ typical of AS and crystalline silicates. This is substantially larger than the $\mathrm{H}-\mathrm{O}-\mathrm{H}$ angle of water (1050), the $\mathrm{C}-0-\mathrm{H}$ angle of alcohols, and the C-0-C angle of ethers (typlcally about 1100).

This large angle suggests a substantial component of sp hybridization in the electronic structure of the oxygen. (Pure sp hybidization would give a bond angle of 1800.) This component of sp hybridation makes dative bond- 
Ing with silicon easier, because it forces the free electron pairs into p-like orbitals, whose shape is optimal for forming dative bonds with silicon. This deduction leads to a useful corollary: If the Si-0-Si bond angle is reduced by externally originated bending, the extent of dative bonding and of a11 its consequences is reduced.

\section{S2.9 The Acid-Base Properties of Sillca Silanols}

The room temperature $\mathrm{pK}_{a}$ of $\mathrm{MSA}$ 1s about 9.8. Since MSA has four equivalent protons, the intrinsic $\mathrm{pK}_{a}$ of any one of these protons is actually $9.8+\log 4=10.4$. This means that MSA is a much more powerful acid than water or the alkyl alcohols. The reason is that dative bonding allows a portion of the negative charge of an lonized sllanolic oxygen to be transferred to the adjacent silicon:

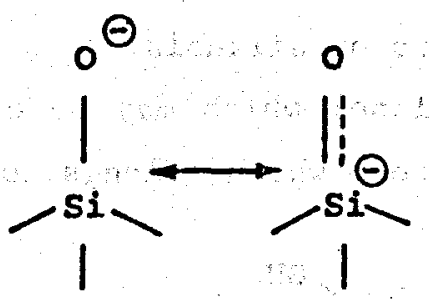

The $\mathrm{pK}_{\mathrm{a}}$ of the surface silanol groups of AS has been spectroscopically estimated to be $7.1 \pm 0.5$ (HaIr and Hert1, 1970). This is in good agreement with numerous estimates based on titration curves which range from about 6.5 to 7.7. (Schindler and Kamber, 1968; Strazhesko et al., 1974; and others.) Clearly, these surface sllanols are even more acldic than MSA. Once again, dative bonding is responsible. An lonized silanol on an AS surface may partake in the following sort of canonical structure which involves two dative bonds:

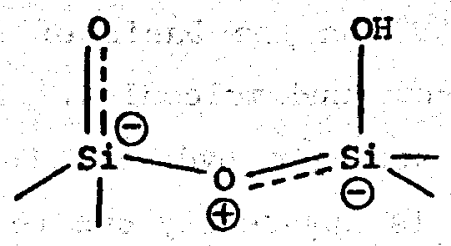


In physical terms, it may be said that the absence (or near absence) of a negative charge on the siloxanic oxygen makes it easier for the silicon to accommodate part of the negative charge of the lonized silanol. Clearly, this cannot happen In MSA which contains only one silicon atom per molecule.

It is likely that the acidity of a silanolic proton varies with the number of siloxanic bonds that its silicon participates in. Thus, the single silanol on a tertiary silicon should be the most acidic, the silanols on a secondary silicon somewhat less acidic, and the silanols on a primary silanol even less so, but st111 more actdic than the sllanols of MSA. It seems very likely that the "Inţrinsic acidity" $\mathrm{pK}_{\mathrm{a}}$ of about 7 determined by titration methods actually reflects the $\mathrm{pK}_{a}$ of the most acidic, 1.e., tertiary silanols. This hypothesis is supported by the observation made by Allen et al. (1971) that the titration curve of an colloidal AS in NaCl media 1s better fitted by a model with two classes of silanolic protons of different acidity than by a model with only one class of silanols.

A fourth kind of surface silanol which may be expected to be chemically distinct is that of a secondary group which belongs to a three-ring:

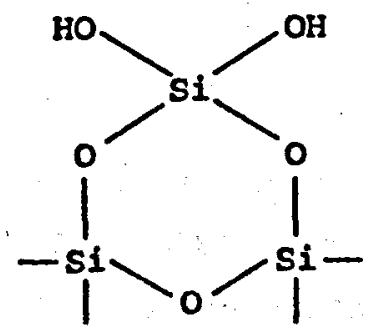

Assuming an $0-S 1-0$ bond angle of 109.50 leads to an S1-0-S1 bond angle of about 130.50. A bond angle this small must certainly reduce dative bonding and, thereby, the acidity of the silanols in this structure.

It is noteworthy that dative bonding does not appear to decrease the basicity of the oxygen in an undissociated silanol group. This conclusion was reached by West and Baney (1959) on the basis of spectroscopic studies of hydrogen bonding among silanol compound molecules. They found that silanol: groups actually seem to form stronger hydrogen bonds among themselves than do alcoholic hydroxyls. This is apparently due to the fact that dative bonding with one silicon can only affect one free electron pair of the oxygen atom but not both. 


\section{S2.10 The First Steps of Silica Polymerization: Dimer to Pentamer}

Engelhardt et al. (1975) have succeeded in interpreting the ${ }^{29} \mathrm{SI}$ MMR spectra of alkaline silicate solutions in terms of the various chemlcal spectes of silicon present. They have since applied NMR spectroscopy to the study of the very first steps of silicic acid polymerization in acid media (Engelhard et al., 1977). A typical experiment was one in which the inftial conditions were 0.5 mole $\mathrm{L}^{-1} \mathrm{MSA}, \mathrm{pH}=2, \mathrm{t}=-2^{\circ} \mathrm{C}$. $\mathrm{pH}$ 's and temperatures In this range were employed In order to make the reaction proceed slowly enough to be able to study it with the limited time resolution of ${ }^{29} \mathrm{~S} 1 \mathrm{MMR}$.

Briefly, they found the first steps of the reaction to proceed via the following NMR-distinguishable species:

- monosilicic acid

- cyclotrisilicic acid

- cyclotetrasiliclc acid

- cyclopentasilicic acid and other secondary s1licon species in rings of five or more members

- more highly condensed species up to and Including bulk AS

They made a point of emphasizing that at no point did they see any sign of disilicic acid or any other form of primary silicon.

We do not see any way to get from MSA to cyclotrisilicic acid other than by way of distliclc acid. The hypothesis that disilicic acid Is the product of the first step of polymerization 18 supported by these authors observation that the initial rate of disappearance of MSA nicely obeys a second order kinetic law.

The reason that disilicic acid was not detected in these experiments is prokably that under these conditions any disilicic actd that is formed quickly rescts with MSA to give cyclotrisiliclc acld. At high MSA concentrations the driving force for this reaction 16 high, because it involves the formation of two new siloxane bonds; In other words, the free energy change for this reaction is probably approximately that for going from MSA to bulk AS. 
A similar argument explains why the cyclic tri- and tetrasilicic acids were observed but not the "1inear" ones: the negative free energy change associated with making an additional slloxane bond by linking together the two ends of the linear form overwhelms the positive free energy term associated with the loss of configurational freedom.

Arguments based on trading off broken bonds for internal rotations are not sufficient to explain the apparently greater stability of secondary silanols relative to primary silanols in cases where the interconversion does not involve a change in the total number of bonds. Consider, for example, the two tetrasilicic acids

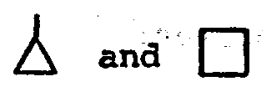

$(2 \cdot 10 \cdot 1)$

Both contain the same number of siloxane bonds, yet the second is the only one that was detected. It must be that the loosening of the ring structure associated with going from the three-ring to the four-ring causes a negative free energy change great enough to compensate for the positive free energy change associated with the loss of the rotational degree of freedom of the primary group.

An analogous argument may be constructed to explain the absence of primary groups attached to more highly condensed structures. In all cases, a primary group would be attached to a tertiary or quaternary group. The latter would be a member of one or more "rings" within the condensed structure. An isolated ring oligomer of four or more units within the aqueous phase might not have any significant ring strain which would cause it to tend to expand at the expense of the attached primary groups. (Although there might well be an "entropic force" favoring ring expansion.) However, the same ring built into a multiply-condensed AS structure very likely would be strained simply because of having the rest of the structure attached to 1t. Therefore, ring expansion at the expense of the primary group would be favored because it would produce a looser, less strained and (probably) higher entropy structure.

This argument explains Engelhardt et al.'s observation that there was apparently no significant number of primary groups attached to even the more highly condensed structures which eventually formed in the course of their experiments. Clearly, the same argument should apply to the AS surface structure as well. 
Unfortunately, Engelhardt et al. were not able to assign specific NMR signals to species beyond the cyclic pentamer.

Somewhat disturbing is the report by Hoebbel and Wieker (1973) that they resolved DSA by paper chromatography in 0.4 molar silicic acid solution after. 5 minutes polymerization at $25^{\circ} \mathrm{C}$ and $\mathrm{pH}=2$. However, they did not unambigously Identify a spot corresponding to cyclo-TSA rather, they assigned a smear between the tetramer and hexamer species to the trimer. It is possible that they mistook the trimer spot for the dimer, and that Engelhardt et al., (1977), were correct in reporting the absence of detectable quantities of dimer. The latter authors themselves suggest this possibility.

Rothbaum and Rohde (197.9) have presented preliminary data on the relative concentrations of mono-, di-, tri-, and tetrasilicic acids in polymerizing solutions of moderate total silica content at temperatures up to $1800^{\circ} \mathrm{C}$ They directly determined the concentrations of these four species by the trimethylsilylation method. Unfortunately; the total molybdate active silica (1.e., the sum of the four species) was not stated, but could be inferred to be roughly equal to the equilibrium solubility of AS at any given temperature plus $0.4 \mathrm{~g} \mathrm{~L}^{-1}$. Making the fairly safe assumption that these small species were roughly in equilibrium among themselves, we reanalyzed this data to obtain approximate values of the molal concentrations of the di-and trisilicic acids that would exist in equilibium with solid AS at any given temperature. The values calculated for the temperatures 90,120 , and $1800^{\circ} \mathrm{C}$ appeared to be consistent and reasonable, and we fitted them with the following equations (concentrations in molal units):

$$
\begin{aligned}
& \log c_{\text {dimer }}=-2.10-775 / \mathrm{T} \\
& \log c_{\text {trimer }}=-3.22-919 / \mathrm{T}
\end{aligned}
$$

To calculate the concentration of the dimer corresponding to a monomer saturation ratio other unity, mult1ply, the value obtained from $(2.10 .2)$ by the square of the saturation ratio; to calculate the concentration of the trimer, multiply the value obtained from $(2.10 .3)$ by the cube of the saturation ratio,

Only an Insigniffcant amount of the tetramer was detected and that only at one temperature. Again, we caution that these are only approximate preliminary values, and should only be used semlquantitatively. However, they are adequate to support our arguments concerning the relative stabilities of the di- and trisilicic acids. Using these formulas, we estimate that the molar 
concentration of the trimer exceeds that of the dimer by about a factor of ten under the conditions employed by Engelhardt et al.

\section{S2.11 Silica Polymerization: Hexamer and Beyond}

A number of oligomeric silicate lons in this range have been identified in various synthetic silicate compounds. These are:

Double-tri-ring hexamer ("triangular prism") (Smolin, 1969)

A heptamer of unknown structure (Hoebbel and Wieker, 1974).

Double four-ring octamer ("cube") (Hoebbel and Wieker, 1971;

Hoebbel and Wieker, 1972; Smol1n et a1., 1972; Smolin et al., 1975, Hoebbel et al., 1976)

Double five-ring decamer ("pentagonal" prism") (Hoebbel et al., 1975).

A number of complex silicate ions have also been isolated in the form of completely reacted trimethylsilyl esters (Hoebbel et al.,1976). Those whose structures have been unambiguously assigned are:

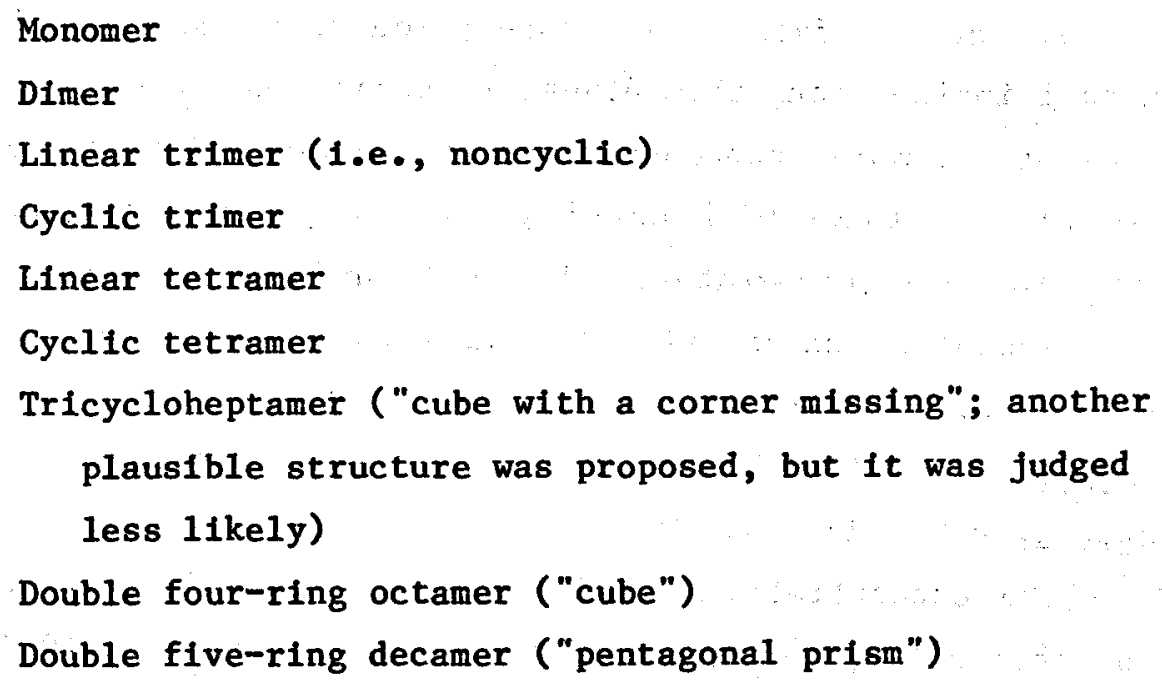

A total of six hexameric species were isolated, and structures were assigned to three of them. A mixture of these six species was prepared by esterifying a synthetic silicate with the composition $\mathrm{N}\left(\mathrm{C}_{2} \mathrm{H}_{5}\right)_{4} \mathrm{OH} \cdot \mathrm{S}_{10} 0_{2} \cdot 10 \mathrm{H}_{2} \mathrm{O}$. The structures of three of these species were determined. These are:

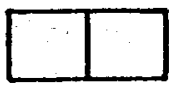

A

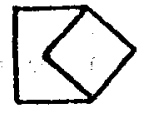

B

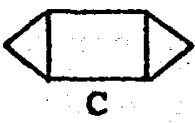

$(2.11 .1)$ 
where each vertex is "silicate" silicon, and oxygen atoms and TMS groups have been ignored.

A specific and completely unsuccessful attempt was made to synthetize the cyclohexasilicic acid ester using Dioptas, a cyclohexasilicate mineral of the formula $\mathrm{Cu}_{6}\left[\mathrm{Si}_{6} \mathrm{O}_{18}\right] \cdot 6 \mathrm{H}_{2} \mathrm{O}$. This observation leads to the inescapable conclusion that cyclohexasilicic acld is unstable relative to the hexamers with structures $A, B$ and $C$. This is to be expected from a simple count of siloxane bonds. It seems safe to generalize this observation slightly, and state that: a silicic acid six-ring will spontaneously be bridged if this is sterically allowed. The results obtained with Dloptas suggest that the formation of one bridging bond (as in structures $A$ and $B$ ) is more 1ikely than the formation of two (as in $\mathrm{C}$ ).

It would be unjustified to claim that all these species play a role in the early condensation of MSA, or even that they ever exist in solution in significant concentrations. For one thing, the stability of silicate lons need not follow the same patterns as that of silicic acid oligomers. To give an obvious example, the disilicate ion $\left(\mathrm{Si}_{2} \mathrm{O}_{7}^{-6}\right)$ is a well-known and stable species, while 1 ts corresponding acid appears to exist only as an ephemeral intermediate. Likewise, the cyclohexasilicate lon is known to exist, while its corresponding acid is unstable. The reason that these lons exist is, of course, that they possess no untonized silanols and, thereby, cannot polymerize or condense any further. Also, a silicate species of middilng stability may exist as part of a crystaline compound simply because of the extra stabilization that the formation of a crystal involves.

St111, there is nothing to suggest that any of the structures identified in the synthetic silicates are Intrinsically unstable. Therefore, we accept that the corresponding polysilicic acids probably all take part in the polymerization of strongly supersaturated MSA solutions, even though some of them may play only secondary roles.

\section{S2.12 The Molecular Mechanism of Silica Polymerization}

Figure 2.1 is a compllation of most of the various known silicic acid oligomer structures discussed and how they were identified. Up to and including the tetramers, this tabulation probably reflects the actual course of 
the early condensation of MSA. Two as yet unobserved but probable intermediate structures ( $4 \mathrm{a}$ and $5 \mathrm{a}$ ) have been included to make this scheme complete. Two other inferred unstable intermediate structures which are known in ester form ( 2 and $3 a$ ) have likewise been included.

However, at $n=5$ the tabulation ceases to be complete; two bridged pentamer structures which do not appear to be unduly sterically strained are possible. These may also exist and play a role in the polymerization process. Several additional hexamers may be constructed, and three of the four tabulated hexamer structures can be assembled in two different isomeric forms. Many more oligomers of $\mathrm{n}=7$ and higher may be constructed.

We conclude that at $n=5$ or 6 the reaction sequence ceases to be unique and many different pathways involving many different structures become possible. It is possible that the maximal extent of condensation attained in the structures $6 \mathrm{~d}, 8 \mathrm{a}$ and $10 \mathrm{a}$ channels most of the early polymerization through them, but truly random structures must become dominant not far beyond. Model building experiments confirm that by $n=15$ or so the structures which occur begin to look like small pieces of amorphous solid rather than large molecules.

These structures and the preceding discussion also suggest the sequence of events involved in MSA deposition on the AS surface.

First, an MSA molecule in solution reacts with a surface silanol group to give a primary sillca group. Attachment to a tertiary silanol is probably kinetically favored (see S2.13).

Second, a rearrangement to a more stable structure occurs. If a silanol is available within reach of the newly attached silica group, a ring of three or more members may be formed. However, model building reveals that this is usually not possible. A more probable fate for the primary group is conversion to a secondary group by a rearrangement of the sort that connects oligomerlc structures $4 a$ and $b$, and $5 a$ and $b$. Such a rearrangement creates or enlarges a "loop" of secondary groups on the surface.

Third, when a "surface 1oop" becomes long enough, it will crosslink internally. The conversion of cyclohexasilicate ion to structures $6 \mathrm{a}$ and $6 \mathrm{~b}$ when protonated is an example of this. Alternatively, a "surface loop" may become long and loose enough to be able to come within bonding range of a silanol attached to some other part of the silica surface. Topologically, such a link is equivalent to bridging a ring. Model building reveals that, 
Figure 2.J

Known and Suspected S1licic Ac1d 01igomer Structures

- 1 known; starting material

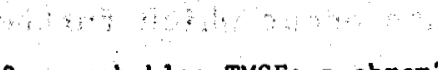

2 probable; TMSE; p-chrom?

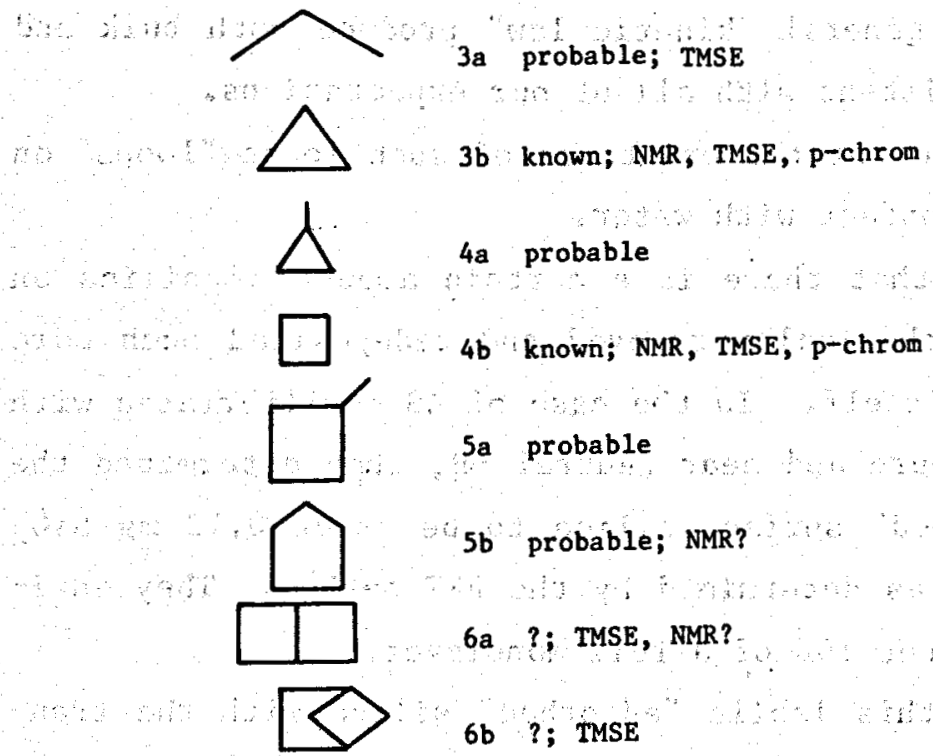


typically, two or three silica groups must be added to a given part of the silica surface to make the formation of a new crosslink sterically possible although one is sometimes sufficient.

Fourth, additional rearrangements occur which further increase crosslinking and reduce steric straln.

Models built following this general "kinetic law" produce both bulk and surface structures which are consistent with all of our expectations.

There is also direct evidence for the existence of such loose "loops" on the surface of AS when it is in contact with water.

Holt and King (1955) found that there is a certain amount of silica on the surface of AS which may be chemically removed and redeposited much more rapidly than can be the bulk AS itself. In the case of AS equilibriated with aqueous solution at room temperature and near neutral $\mathrm{pH}$, they determined the amount of this labile or "adsorbed" surface silica to be about $0.13 \mathrm{mg} \mathrm{\textrm {SiO } _ { 2 }}$ per square meter of surface area as determined by the BET method. They estimated that this corresponds to about $16 \%$ of a full monolayer.

It is tempting to identify this labile "adsorbed" silica with the transient population of primary groups and the "loose" secondary groups that the former are approximately in equilibrium with. These molecules are kinetically distinct in that their creation from MSA molecules in solution involves the formation of only one additional siloxane bond. They may well be expected to be relatively labile.

Clearly, the overall mechanism and rate law for molecular deposition and dissolution at the AS surface must involve "adsorbed" silica as an intermediate species. This was first proposed and discussed in detail by stöbèr (1967).

\section{S2.13 The Base Catalyzed Condensation Reaction}

The polymerization and depolymerization of silicic acid are catalyzed by hydroxide ion (Hurd et al., 1934). This is usually the dominant reaction above about $\mathrm{pH} 3$ (Iler, 1952). Between about $\mathrm{pH} 3$ and 7 the reaction is first order in hydroxide concentration. At about $\mathrm{pH} 8$ or 9 the rate of polymerization goes through a maximum, and decreases at higher pH's (Marsh et al., 
1976). The rate of depolymerization reaches a maximum limiting value at about $\mathrm{pH} 11$ but does not decrease at higher $\mathrm{pH}$ (Greenberg, 1957).

It is believed that the actual condensation step is a reaction between an Lonized silanol and a silica group none of whose silanols are lonized. The microscopic mechanism is very likely to be $S_{N} 2$ with steric inversion:

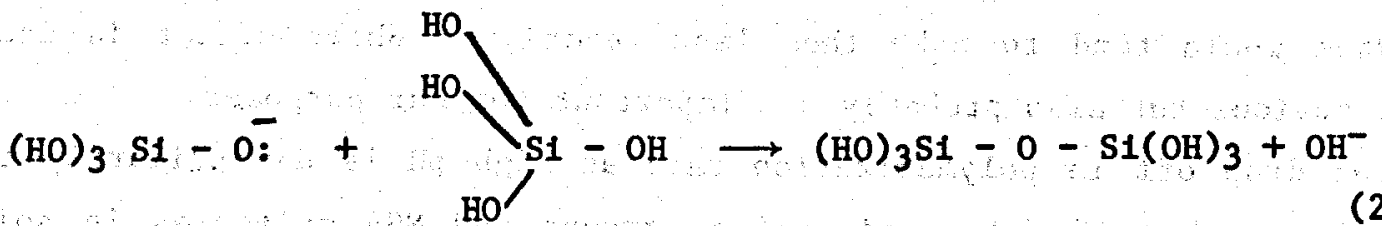

This sort of "backside attack" is the rule with carbon compounds and is also known to occur with various organosilicon compounds in both polar and nonpolar media (Sommer, 1965, pp. 66-72). In nonpolar media frontside $\mathrm{S}_{\mathrm{N}}{ }^{2}$ attack with retention of configuration is also known to occur (op.cit, pp. 51-56). Frontside attack may also occur when steric constraints prevent the Inversion associated with backside attack (op cit., pp. 154-158). This situation may arise with tertiary silicons on the As surface, but backside attack seems likely to be the more important by far.

The more acidic a silanol is, the more likely it is to be dissociated and avallable to serve as the nucleophile. Very probably, the surface silanols attack free MSA when the latter first attaches itself to the surface rather than the other way around. Also, tertlary surface sllanols are likely to be favored as attachment points because of their greater acidity.

It is possible that the more basic of the lonized silanols are better nucleophiles. In the case of a series of $\mathrm{S}_{\mathrm{N}}{ }^{2}$ reactions at a carbon center, each of which involves a single substrate and a series of related nucleophiles, there is often observed a linear relationship between the logarithm of the rate constant and the $\mathrm{pK}_{\mathrm{a}}$ 's of the nucleophiles' conjugate acids. The slope is always between zero and one (Weston and Schwarz, pp. 191-195). As a general rule, the value of such a regression coefficient depends on the extent to which the transition state resembles elther reactants or products. If the transition state resembles the products, the effect of the detailed properties of the reactants upon rate is usually small, and vice versa. Because hydroxide ion is a far more powerful base than an lonized silanol, the transition state 
must closely resemble the products in this case. We conclude that the nucleophilic reactivities of different lonized surface silanols may be assumed to be equal for practical purposes.

Such a correlation may also be expected in the case of substrate reactivity. The greater electrophilicity of more highly condensed silicon atoms should tend to make them more reactive. On the other hand, greater steric hindrance would tend to make them less reactive. Which effect is dominant is not obvious but also probably not important for our purposes.

The drop off in polymerization rate at high $\mathrm{pH}$ is due primarily to the decreasing number of unionized surface groups and MSA molecules in solution available to serve as substrates. It is the kinetic expression of the increase In solubility at high $\mathrm{pH}$ (see $\mathrm{S2.2}$ ). The attainment of a limiting depolymerization rate above $\mathrm{pH} 11$ may be due to a transition to diffusion control. Another effect which tends to slow both reactions above $\mathrm{pH} 7$ is that of Increasing surface charge. This increase causes the concentration of lonized surface silanols and of hydroxide at the surface to lag behind the hydroxide concentration in the bulk solution as the $\mathrm{pH} 15$ increased.

An ionized silanol group on the silica surface may be "bare" (as depicted above), or it may have a cation bound to it. Our own work has demonstrated that both bare and cation-paired lonized silanols (at least in the case of sodium and other univalent cations) contribute to the reaction rate, and contribute equally in as far as we can determine from our kinetic data.

Adding dissolved salts to the medium increases the surface density of cation-paired ionized silanol groups at any given $\mathrm{pH}$ value. This is why adding salts accelerates the deposition of MSA on preexisting surfaces. (Added salts accelerate homogeneous nucleation by an even greater factor because they also decrease the solubility of silica and, thereby, increase the supersaturation rat1o. They also lower the surface tension of the AS-water interface, and this accelerates nucleation even further.)

\section{S2.14 Catalysis by Hydrogen Fluoride and Hydrogen Ion}

At about $\mathrm{pH} 2$ the rate of silica polymerization passes through a minimum, and again increases at still lower $\mathrm{pH}$. Iler (1952) demonstrated that in this $\mathrm{pH}$ range even very small concentrations of fluoride salts ( $3.0 \mathrm{E}-4$ molar) 
greatly accelerate the rate of polymerization. He concluded that the apparent "co-catalysis" by hydrogen lon and fluoride ion 1s, in reality, simply catalysis by hydrofluoric acid (HF). He further suggested that what appears to be catalysis by hydrogen ion alone is really catalysis by trace amounts of HF. Working with inftially 1 molar MSA solutions at $25^{\circ} \mathrm{C}$ and $\mathrm{pH}^{\prime} \mathrm{s}$ near 2 , Iler found that $2 \mathrm{E}-3 \mathrm{M}$ HF decreased the gel time by about a factor of 100 . However, an Increase of $\mathrm{HF}$ to $5 \mathrm{E}-3 \mathrm{M}$ further decreased the gel time by only about a factor of 2. With $1 \mathrm{E}-4$ and $3 \mathrm{E}-4 \mathrm{M} \mathrm{HF}$, there was a maximum in gel time at about $\mathrm{pH} 1.6$. With $1,1 \mathrm{E}-3$ and $2.1 \mathrm{E}-3 \mathrm{M} \mathrm{HF}$ no $\mathrm{pH}$ dependence was observed. Adding aluminum in millimolar amounts counteracted the catalytic effect of HF. Iler attributed this to the removal of HF by complex formation.

Tal and chen (1965) studied the effect of added KF upon the gel time. In their experiments the total fluoride concentration ranged from $0.0045 \mathrm{M}$ to $0.8 \mathrm{M}$. Their results varied somewhat depending on the silica concentrat1on. Using waterglass with Na $20 / \mathrm{SiO}_{2}=3.36$ and total $\mathrm{S}_{2}=0.870 \mathrm{M}$, or sodium metasilicate with total $\mathrm{SiO}_{2}=0.723 \mathrm{M}$, they observed a dramatic effect on the gel time at $\mathrm{pH}$ values below 3 , and a smaller but still observable effect up to about $\mathrm{pH}$ 5. At KF concentrations up to about $0.05 \mathrm{M}$, the gel time decreased with increasing concentration of $\mathrm{KF}$. Between 0.05 and $0.10 \mathrm{M} \mathrm{KF}$, the gel time was unaffected by the variation in $\mathrm{KF}$. Above $0.10 \mathrm{M}$ $\mathrm{KF}$, the gel time again Increased with increasing KF. All of these effects decreased rapidly with increasing $\mathrm{pH}$ above about $\mathrm{pH} 3$. These, results suggest catalysis by $\mathrm{HF}$ at low $\mathrm{pH}$ and catalysis by $\mathrm{F}^{-}$at higher $\mathrm{pH}$. The effect of the latter mechanism is less striking because it takes place in a pH range in which there is strong competition from the common "OH catalyzed" mechanism. At high concentrations of $\mathrm{KF}$, the solubility of silica is sufficlently increased by the formation of fluoride complexes to outweigh and reverse the accelerating catalytic effect.

Using sodium metasilicate solutions with $\mathrm{SiO}_{2}=0.1533 \mathrm{M}$, TaI and Chen observed an essentially $\mathrm{pH}$ independent accelerating effect which persisted at least up to $\mathrm{pH} 9$, and Increased steadily with increasing $\mathrm{kF}$. In this case, it appears that the accelerating effect was due mostly to increased surface silanol Ionization caused by the addition of the potassium ion rather than to specific catalysis by fluoride. This effect becomes important only at lower 
$\mathrm{SiO}_{2}$ concentrations at which the added potassium from the $\mathrm{KF}$ represents a greater proportional increase in total cation concentration and lonic strength. Tai and Chen also determined the fraction of the added fluoride that remained chemically bound to the gel that was produced. They found that the fraction of bound fluoride varied from unity at $\mathrm{pH}-0.15$ to zero at $\mathrm{pH} 5.71$. Working with $1 \mathrm{~g} \mathrm{~L}^{-1} \mathrm{SiO}_{2}$ solutions ( $\left.0.017 \mathrm{M} \mathrm{MSA}\right)$ at $\mathrm{pH} 7$ and $30^{\circ} \mathrm{C}$ Baumann (1959) found that NaF did not effect the rate of polymerization at concentrations below about $0.01 \mathrm{M}$. At higher concentrations of NaF, the rate decreased. $\mathrm{NaF}$ had no effect on the depolymerization reaction below $10^{-3} \mathrm{M}$, but accelerated it at higher concentrations. Baumann interpreted these effects as being due to the increase in silica solubility associated with silicon hexafluoride complex formation.

In their depolymerization experiments, Stade and wieker (1971) found that at $25^{\circ} \mathrm{C}$ and $\mathrm{pH} 5.5 \mathrm{~F}^{-}$in concentrations as small as $5 \mathrm{E}-7 \mathrm{M}$ had a noticeable accelerating effect. The increase in rate appeared to be inear in $\mathrm{F}^{-}$concentration up to about $3 \mathrm{E}-6 \mathrm{M}$, but ceased to increase with further addition of fluoride. The total increase observed was about 45\%. This "saturation effect" remains to be explained if it is real. It may or may not be related to the superficially similar phenomenon observed by $\mathrm{Tat}$ and Chen at much higher fluoride concentrations. It is not clear whether this catalytic effect was due to $\mathrm{F}^{-}$or to the small amount of HF still present at this $\mathrm{pH}$. Stade and Wieker found that $2 \mathrm{E}-5$ molar $\mathrm{NH}_{4} \mathrm{~F}$ had a large effect upon the activation energy of the reaction up to about $\mathrm{pH}$ 4. Because the $\mathrm{pK}_{\mathrm{a}}$ of $\mathrm{HF}$ is 3.45 (at $25^{\circ} \mathrm{C}$ ), they interpreted this to mean that $\mathrm{HF}$ is the major catalytic species rather than $F^{-}$. They dispute Iler's contention that acid catalysis of silica reactions is generally due to catalysis by traces of $\mathrm{HF}$, arguing that if this were the case, adding further small amounts of HF would not effect the activation energy.

Taken as a whole, these results make an excellent case for the catalytic effect of HF. Catalysis by $\mathrm{F}^{-}$is not as certain, but it seems 1ikely. Baumann's results and those of Tal and Chen indicate that both mechanisms can probably be ignored above about $\mathrm{pH} 6$.

The only mechanism that seems consistent with catalysis by HF rather than $F^{-}$is one in which an uncharged complex of MSA and HF plays the role of an unstable intermediate (Strelko, 1970). A likely first step is: 


$$
\mathrm{HF}+\mathrm{SI}(\mathrm{OH})_{4} \rightarrow \mathrm{SI}(\mathrm{OH})_{3} \mathrm{~F}+\mathrm{H}_{2} \mathrm{O}
$$

(Iler suggests that such an Intermediate may be six coordinated. Assuming a structure in which the silicon is also coordinated by two water molecules would not effect our argument in any way The intermediate could also be five coordinated with the formula $\left.\mathrm{Si}(\mathrm{OH})_{4} \mathrm{FH}.\right)$

Because $H F$ is a far stronger acid than water, $F^{-}$may be expected to be a far better leaving group. Written for the particular case of the dimerization of MSA, the actual condensation step would then be:

$$
\mathrm{MSA}+\mathrm{SI}(\mathrm{OH})_{3} \mathrm{~F} \longrightarrow \mathrm{DSA}+\mathrm{HF}
$$

The overali rate law for polymerization via this mechanism would be first order in HF concentration.

There are two plausible mechanisms that would give a overall rate law that is first order in the concentration of $F^{-}$. The first is the formation of an uncharged monofluoride complex (Eq. 2.14.1) followed by nucleophilic attack by an lonized silanol group:

$$
\mathrm{Si}(\mathrm{OH})_{3} \mathrm{O}^{-}+\mathrm{S} 1(\mathrm{OH})_{3} \mathrm{~F} \longrightarrow \mathrm{DSA}+\mathrm{F}^{-}
$$

The second is the formation of an anionic monofluoride complex, $\mathrm{SI}(\mathrm{OH})_{4} \mathrm{~F}^{-}$, followed by nucleophilic attack by an unionized silanol:

$$
\mathrm{Si}(\mathrm{OH})_{3} \mathrm{OH}+\mathrm{Si}(\mathrm{OH})_{4} \mathrm{~F}^{-} \rightarrow \mathrm{DSA}+\mathrm{F}^{-}+\mathrm{H}_{2} \mathrm{O}
$$

In all cases, the concentration of both HF and $F^{-}$would be controlled by the dissoctation equilibrium of $\mathrm{HF}$.

Iler's own results appear to be inconsistent with his hypothesis that the apparent hydrogen Ion catalyzed pathway is actually catalyzed by trace amounts of HF. Without added $F$ he observed a maximum in the gel time (i.e., a minimum in the rate of polymerization) between $\mathrm{pH} 1$ and 2 . With $1.1 \mathrm{E}-3$ molar added $\mathrm{F}$ and above, the gel time was much smaller and independent of $\mathrm{pH}$ (in this range). 
The latter observation is consistent with HF catalysis, because the concentration of $\mathrm{HF}$ is independent of $\mathrm{pH}$ in this range. The former observation is consistent with the hypothesis of $\mathrm{H}^{+}$catalysis below the gel time maximum and $\mathrm{OH}^{-}$catalysis above it. When $\mathrm{F}$ is added, both of these are swamped by the HF catalyzed mechanism.

The most likely microscopic mechanism for $\mathrm{H}^{+}$catalysis is

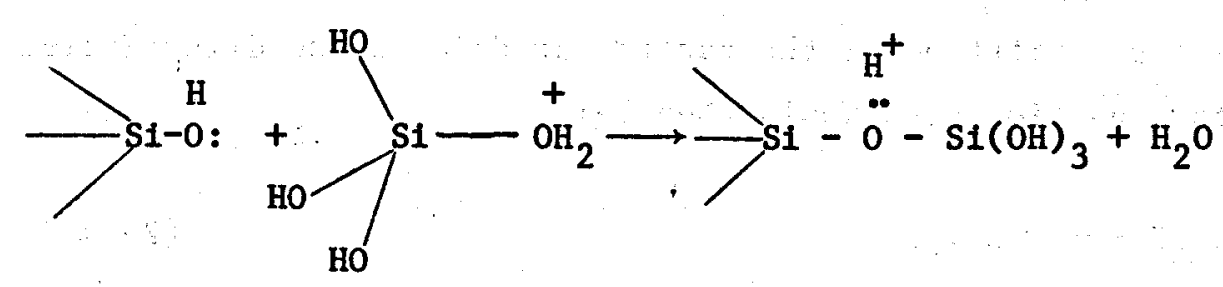

followed by rapid deprotonation.

Although we believe that such a mechanism actually does exist, we doubt that it is at all significant compared to the HF and $\mathrm{OH}^{-}$catalyzed mechanisms under practical conditions.

Both the $\mathrm{HF}$ and $\mathrm{H}^{+}$mechanisms involve the transitory existence of a protonated siloxanic oxygen immediately following the nucleophilic attack. The $\mathrm{H}^{+}$mechanism also involves a protonated silanol (1.e., water) which serves as the leaving group. Clearly, reactions which involve and produce structures with more basic oxygens will be favored. It is known that higher degrees of condensation favor dative bonding, and that dative bonding reduces basicity. Thus, more highly condensed structures may be expected to be less reactive under efther of these mechanisms (opposite to the case, with $\mathrm{OH}^{-}$ catalysis). This means that the formation of small ollgomers and very loose, secondary group rich surface structures will be favored by both HF and $\mathrm{H}^{+}$catalysis. This is precisely what has been observed (Engelhardt, et al., 1977; Iler, 1973, pp. 9-11; Acker, 1969). 


\section{S2.15 Other Catalysts}

Stade and Wieker (1971) have studied the effects of a whole serles of nucleophilic substances upon the rate of depolymerization of silicic actd sols. They analyzed their results using the expression

$$
\text { rate }=k_{x}+k_{n}(N)
$$

where $K_{x}$ represents the rate of the "uncatalyzed" (i.e., $\mathrm{OH}^{-}$catalyzed reaction), (N) is the concentration of the nucleophile, and $k_{n}$ is the "catalytic coefficient". Their values for $K_{x}$ and the various $k_{n}$ are given in Table 2.4 . Where necessary, the concentration of the actual species of interest was calculated from the concentration added and the corresponding dissociation equilibrium.

Stade and wieker reported that catalysis by $\mathrm{HF}$ and hydroxylamine showed a "saturation effect", $1 . e$. , the rate of reaction ceased to increase with concentration of catalyst beyond a certain point. They reported the threshold concentration for HF to be 3E-6 molar, but did not report a value for hydroxylamine and $\mathrm{F}^{-}$. The reaction mechanisms involving catalysis by the other substances in Table 2.4 are probably similar to one or another of the mechanIsms proposed for the fluoride catalyzed mechanisms. General nucleophilic catalysis analogous to (2.14.3) seems iikely in most cases. Aside from HF, none of these substances is likely to be present in geothermal brines in concentrations large enough to cause a noticeable effect.

The sizeable catalytic coefficients of the two amines suggest that ammonia may also be an effective catalyst. Bisulfide Ion (HS-) and bicarbonate are two other nucleophiles known to exist in geothermal brines in significant concentrations. All three at least deserve to be screened for catalytic activity.

\section{S2.16 Toward Predicting the Rates of Molecular Deposition}

Literally the only published datum relating to the actual, absolute rate of deposition per unit surface area is the statement by Iler (1973, p.15) that, at $100^{\circ} \mathrm{C}$, the maximum rate at which MSA may be deposited on the surface of 
Table 2.4

Catalytic Coefficients for Various Nucleophilic Catalysts of the Silica Depolymerization Reaction

Data of Stade and Wieker (1973)

$$
\begin{aligned}
& \mathrm{T}=25^{\circ} \mathrm{C} \\
& \mathrm{pH}=5.5 \\
& \mathrm{~K}_{\mathrm{x}}=0.014 \mathrm{~min}^{-1}
\end{aligned}
$$

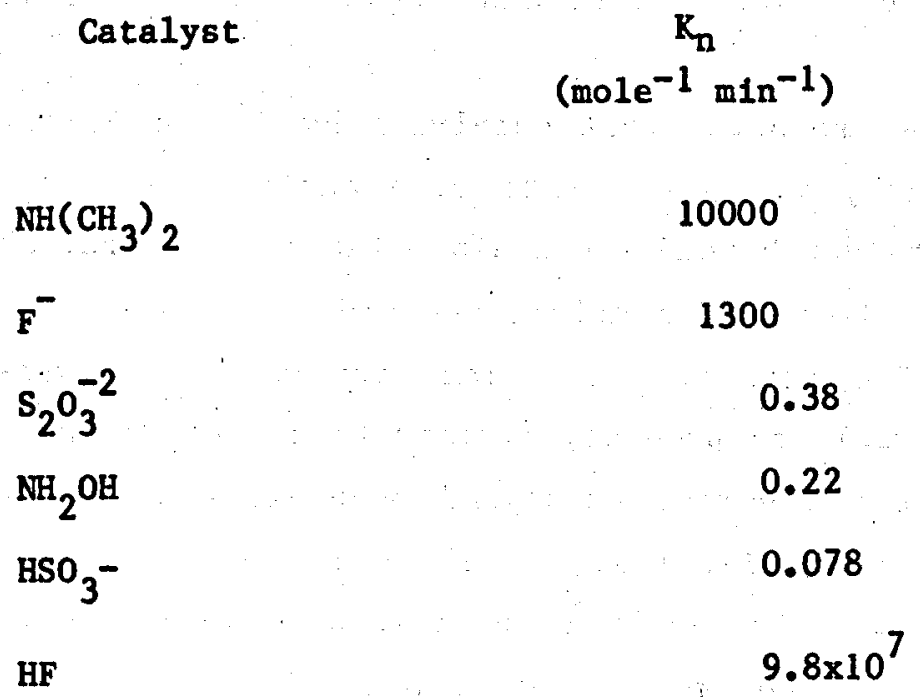


preexisting colloidal particles without nucleating new ones is about $0.005 \mathrm{~g}$ $\mathrm{m}^{-2} \mathrm{hr}^{-1}$. This is equivalent to a rate of surface advance of $2.3 \mathrm{~nm} \mathrm{hr}^{-1}$. Iler has elsewhere stated (Iler, 1975) that this datum refers to $\mathrm{pH}$ 9. We estimate that the MSA concentration under the conditions referred to was about $0.7 \mathrm{~g} \mathrm{SiO}_{2} \mathrm{~kg}^{-1}$.

In his thesis Rimstidt (1979) has reported the results of numerous dissolution experiments using $A S$, quartz and cristobalite over a wide range of temperature. A few precipitation experiments were reported as we11. The experiments were performed using nominally salt free media; 1.e., water with nothing but MSA dissolved in 1t. The data was fitted using a first order rate law. The values found for the rate constant for the dissolution reaction over the temperature range 0 to $300^{\circ} \mathrm{C}$ were approximately fitted by the formula

$$
\log k_{-}\left(m^{-2} s^{-1}\right)=-0.707-2598 / T \quad(2.16 .1)
$$

This corresponds to an activation energy of $11.9 \mathrm{kcal}$ mole $\mathrm{e}^{-1}$. There - was no significant difference between the rates of dissolution of the different s111ca phases.

Unfortunately, Rimstidt apparently did not at tempt to control or even to record the $\mathrm{pH}$ values in his experimental media. This makes his results very hard to relate to other conditions, and is probabiy in large degree responsible for the large scatter in his rate constant values (slightly over an order of magnitude at any given temperature). All this conspires to make his results useful for purposes of prediction only in a semiquantitative sense.

Rimstidt also tried to calculate molecular deposition rate constants from his dissolution data using the Law of Microscoplc Reversibility. The values thus obtained are probably even less reliable and useful because there is no reason to assume that the Law of Microscoplc Reversibility in its simplest form applies to reactions at solid surfaces. (Indeed, our own kinetic results clearly show that it does not:) For this reason these derived "precipitation rate constants" are not quoted here.

Aside from the above, all that is avallable in the literature are a few scattered values of apparent rate constants, activation energies, etc.

Gato (1956) suggested that the rate of decrease of dissolved stilca concentration under conditions of homogeneous nucleation could be described by an apparent third order rate law of the form 


$$
d c / d t=k\left(c-c_{0}\right)^{3}
$$

but did not justify the assumption. Alexander (1953) found an apparent third order rate law at $\mathrm{pH}=1.7$ and 2.1 , and a second order 1 aw at $\mathrm{pH}=4.36$. Bauman (1959) reported apparent rate orders of between 2 and 5 , depending on the $\mathrm{pH}$ and initial concentration. Hurd and Sheffer (1941) reported an apparent order of either 2 or 3. (The choice depends on whether one assumes that gelation occurs after a certain amount of colloldal AS has formed, or after a certain fraction of the initial MSA has condensed.) Unfortunately, none of these investigators made any attempt to distinguish between actual chemical kinetics and the kinetics of nucleation. It is very likely that their results are influenced as much by the latter as by the former and are, therefore, of questionable use for our purposes.

Jorgensen (1968) avoided this problem by studying the growth and dissolution of preformed colloidal particles of AS. He was able to achieve a reasonable fit by using Gato's "cubic" formula above. Friedberg (1955) also used a preformed collold, but did not attempt to fit his results analytically.

On the basis of gel time determinations, Penner (1946) reported the following values for the activation energy for the acid and base catalyzed polymerization mechanisms:

$$
\begin{aligned}
& \mathrm{E}_{\mathrm{H}^{+}}=9.7 \mathrm{kcal} \mathrm{mole}^{-1} \\
& \mathrm{E}_{\mathrm{OH}^{-}}=16.1 \mathrm{kcal} \mathrm{mole}-1
\end{aligned}
$$

Other investigators have reported similar values (Hurd and Barclay, 1940; Hurd and Letteron, 1932; Hurd and Schuyler-Miller, 1932).

Stade and Wieker (1971) reported an activation energy of 8.6 or $8.7 \mathrm{kcal}$ mole $e^{-1}$ for the HF catalyzed dissolution reaction.

Some work has also been done on the dissolution of AS in alkaline media (Greenberg, 1957; $0^{\prime}$ Connor and Greenberg, 1958). The latter authors found that the rate of dissolution was proportional to the surface area and had an activation energy of $18 \pm 0.2 \mathrm{kcal} / \mathrm{mole}$. This value $1 \mathrm{~s} 1.9 \mathrm{kcal}$ mole $\mathrm{k}^{-1} \mathrm{greater}$ than the activation energy reported by Penner (1946) for the polymerization reaction. Fournier and Rowe (1977) have found the differential heat of solution of AS to be $3.71 \pm 0.05 \mathrm{kcal} \mathrm{mole}^{-1}$. The fact that the difference 
between the two reported activation energies is one-half of this value is consistent with our assumption that the rate determining step of either reaction is the making or breaking of a single siloxane bond.

Wirth and Gieskes (1978) have reported extenstve work on the rate of dissolution of vitreous silica in alkaline media containing $\mathrm{NaCl}$ and $\mathrm{MgCl}_{2}$ at room temperature. They found a good correlation between dissolution rate and surface charge density. Surprisingly, the dissolution rate varied as the square of the charge density.

All in all, we found the state of the literature data In this area to be such that we had no alternative to initiating our own experimental program.

\section{S2.17 The Nucleation of Colloidal Amorphous Sillca}

The preceding discussion has been largely limfted to the case of MSA deposition on a pre-existing AS surface. It, 1 s applicable to molecular deposition on most other types of surfaces as well, as most are readily converted to AS surfaces on contact with qupersaturated MSA solution. Very roughly speaking, such deposition proceeds at a rate on the order of nanometers per hour. It is easy to determine that this process is far too slow to account for the formation of scale at an observable rate. The obvious inference is that most of the excess MSA In solution is actually deposited on minute colloidal particles suspended in the brine. These then adhere to macroscopic surfaces and cause either silica scale or a compact silica gel to form. (The foregoing based mostly on Iler, 1975).

The initial formation of the colloidal AS particles is a process of nucleation similar to those In other systems. At the high initial supersaturation ratios required for rapid polymerization, the so-called homogeneous nucleation process is dominant. This was first convincingly demonstrated and clearly stated by A. Makrides, W. W. Harvey and their coworkers (Harvey et a1., 1976, Makrides et al., 1978). These authors worked at between 75 and $105^{\circ} \mathrm{C}$. Experiments very similar to theirs were earlier performed at $30^{\circ} \mathrm{C}$ by Baumann (1959). He obtained very similar results, but, unfortunately, falled to recognize the homogeneous nucleation phenomenon in them. Rothbaum and Rohde (1979) have recently obtained similar data at temperatures up to $180^{\circ} \mathrm{C}$, but also did not recognize the true significance of the characteristically shaped curves they obtained. 
No preexisting nucleus is involved in the homogeneous nucleation process. Rather, a few small oligomers grow by what is essentially a random fluctuation process until they reach so-called critical nucleus size, beyond which they grow rapidly as would any externally supplied colloidal particle.

The rate of homogeneous nucleation is largely determined by the free energy of the critical nucleus and by the rate of molecular deposition on its surface. We will speak in terms of the so-called Lothe-Pound Theory of Homogeneous Nucleation (see the extensive discussion in Abraham, 1974). The Lothe-Pound theory is a straightforward and logical extension of the so-called "Classical" Theory of Homogeneous Nucleation, but avoids the well known major deficiency of the latter. In the particular case of AS colloid nucleation, a fortultous physical circumstance makes the Lothe-Pound theory as simple and easy to work with as is the classical theory. Indeed, the simple and transparent form of the classical theory is preserved almost unchanged.

The "classical" expression for the free energy of formation of the critical nucleus from dissolved silica is:

$$
\Delta F^{*}=16 \pi / 3 \quad \gamma^{3} /\left(\rho_{n} k_{n} T_{B} \ln s\right)^{-2}
$$

where

$$
\begin{aligned}
\rho_{n} & =\text { the density of solid AS in } \mathrm{SiO}_{2} \text { units } \mathrm{cm}^{-3} \\
& =2.21 \mathrm{E} 22 \mathrm{~cm}^{-3} \\
S & =c / c_{0} \\
\gamma & =\text { the surface tension of the AS-water interface }
\end{aligned}
$$

The radius and area of the critical nucleus are:

$$
\begin{aligned}
& r^{*}=2 Y /\left(\rho_{n} k_{B} T \ln S\right) \\
& A^{*}=4 \pi r^{* 2}=3 \Delta F^{*} / \gamma
\end{aligned}
$$

The expression for the steady nucleation rate derived from the LothePound Theory is

$$
I_{N}=Z A^{*} R_{m d} \rho_{n} Q_{L P} \exp \left(-\Delta F^{*} / k_{B} T\right)
$$

where $I_{N}$ is the nucleation rate expressed in units of $\left(\mathrm{kg} \mathrm{H}_{2} \mathrm{O} \mathrm{min}\right)^{-1}$ and 


$$
\begin{aligned}
& R_{m d}=\text { the rate of molecular deposition in } \mathrm{g} \mathrm{S1O} \mathrm{min}^{-1} \mathrm{~cm}^{-2} \\
& \mathrm{Z} \text { = the "Zeldovich factor", a dimensionless number which is } \\
& \text { typically between } 0.01 \text { and } 0.1 \\
& \text { QLP = the "Lothe-Pound factor" }
\end{aligned}
$$

$\Delta F^{*}$ is the free energy change associated with creating a stationary colloidal particle of critical nucleus size. (This $1 \mathrm{~s}$ all that is constdered in the Classical Theory of Nucleation.) The insertion of the Lothe-Pound factor corrects the value of the calculated nucleation rate for the fact that the critical nucleus is actually in motion: it diffuses through the water, and it executes rapld "jiggling motions" of both translational and rotational nature on a smaller scale in time and space. These degrees of freedom greatly decrease the free energy of formation of the critical nucleus. Our own work has demonstrated that $Q_{L P}$ is approximately constant (in this particular case) and equal to $3.34 \mathrm{E} 25\left(\mathrm{~kg} \mathrm{H}_{2}\right)^{-1}$. The physical significance of this number is that it is equal to the number of water molecules in a kilogram of water, and, thereby, approximately equal to the number of positions available for the critical nucleus to occupy in a volume of space that contains a kilogram of 11quid water. The fact that the value of $Q_{L P}$ is known and is approximately constant is the great simplifying consideration alluded to above. The derivation of this result is presented in 53.7 .

$A^{*} R_{m d} \rho_{n}$ is the rate of monomer deposition on the critical nucleus. $z$ is introduced to account for the facts that the actual concentration of critical nuclei is smaller than the "equilibrium" concentration, and that critical nuclei may decrease in size as well as Increase.

Equation (2.17.4) shows that the value of $I_{N}$ Is determined by the value of $\Delta F^{*}$. Equation $(2,17.1)$ shows that $\Delta F^{*}$ is, in turn, determined by $\gamma$ and $S$. Because of the exponential form of $(2.17 .4)$, the value of $I_{N}$ is profoundly affected by the values of these two physical parameters. Under typical homogeneous nucleation conditions ( $S$ between 2 and 5 ) a change in the value of $S$ by one usually changes the rate of nucleation by several orders of magnitude (op.cit., pp. 253-254). The variation of $I_{N}$ with $S$ is so great that there is an apparent "threshold value" of $S$, below which no nucleation is observed and above which nucleation occurs rapidly. In the case of supersaturated vapors, the critical value of $S$ is generally between about 2 and 5 . 
There is evidence for such a "threshold value" of $S$ in the case of AS nucleation as well. For example, working at $95^{\circ} \mathrm{C}$, Makrides, et al. (1978) did not study the rate of nucleation below about $S=2.1$. Although they do not say so, the apparent reason for this is that at lower values of $S$ the rate of nucleation becomes too low to be conveniently studied. In his similar experiments performed at $30^{\circ} \mathrm{C}$, Baumann (1959) did not work below about $S=3.3$, again apparently because of the rapid fall-off in the "rate of polymerization".

Iler (1973, p.15, 1975) reports that there is a certain maximum rate at which MSA may be deposited on colloidal AS. If an attempt is made to add MSA more rapidiy to the solution in order to exceed this rate, the result is nucleation of new particles in addition to the growth of preexisting ones. This "critical growth rate" phenomenon is easily interpreted as being a consequence of the "threshold supersaturation" effect.

The field evidence for "threshold supersaturation" is mixed. Rothbaum and Anderton (1975) have reported the MSA concentration in. "mixed Wairake1 brine" at $90^{\circ} \mathrm{C}$ to drop from an initial value which corresponds to $S=1.75$. (This could be due to heterogeneous nucleation.) In their studies of silica rich cooling tower water at 32 to $38^{\circ} \mathrm{C}$, Midkiff and Foyt (1976 and 1977) found that the sequestration of calcium prevented visible silica precipitation below about $S=1.5$, but not above about $S=2.5$. However, their results also seem to be consistent with the hypothesis that the silica stays in suspension as a stable colloid in the absence of calclum.

It is probable that, in practice, homogeneous nucleation with all of its characteristics dominates if the initial supersaturation ratio is high enough for homogeneous nucleation to be rapid, while heterogeneous nucleation is dominant with lower initial supersaturation ratios.

\section{S2.18 The "Induction Time" for Nucleation and the Value of the Surface Tension}

An "Induction" or "lag" time is often associated with the nucleation process. Characteristically, there is some perlod of time during which the concentration of monomer remains constant and nothing seems to happen. Finally, the concentration of monomer begins to decrease, and this is indicative of the nucleation process. In most physical systems, the induction time is 
largely a theoretical concept because it is too short to observe. In the case of the nucleation of colloidal AS, the induction time is long enough to be easily observed and is usually obvious in the kinetic data. (Indeed, the convenient time scale and relatively good experimental reproduclbility of the nucleation process in this case makes AS the Ideal physical system with which to study homogeneous nucleation in deta11.)

There are two physical interpretations of the induction time phenomenon. It is likely that one reflects physical reality in some systems, while the other reflects it in others.

The first interpretation is that the Induction time reflects the amount of time required for the rate of nucleation to build up to a "steady state" value. (Actually, the rate builds up, peaks, and then falls off again as the value of $S$ drops as a consequence of nucleation and particle growth). This induction time is roughly the time required for subcritical clusters to grow to critical nucleus size and slightly beyond it. It is longer at lower values of $\mathrm{S}$, since the critical nucleus size is greater at lower $\mathrm{S}$ (see Eqs. 2.17.2 and 2.17.3). Induction times of this sort are known to be very small in the case of vapor condensation (Abraham, 1974, pp.91-101), but can be significant in solid state precipitation reactions (Russe11, 1968 and 1969). This interpretation applies to homogeneous nucleation only.

The second Interpretation is that the induction time is simply the length of time required for enough particles to nucleate and grow to the point that the monomer concentration is noticeably affected. It is implicit in this Interpretation that the "steady state" nucleation rate ls attained so rapidly that the fact of an initially slower nucleation rate may be ignored for practical purposes. This interpretation applies to induction times observed in the case of homogeneous and heterogeneous nucleation both. Interpreted in this way, the induction time is simply related to the monomer deposition rate $R$ and the steady state nucleation rate $I_{N}$ it varies in approximate proportion to

$$
\left(\mathrm{R}_{\operatorname{md}} \rho_{\mathrm{n}}\right)^{-1}\left[\mathrm{I}_{\mathrm{N}} /\left(\mathrm{R}_{\mathrm{md}} \rho_{\mathrm{n}}\right)\right]^{-1 / 4}
$$

Th1s result was first obtained by Johnson and O'Rourke (1953), and then rederived and applied to the case of AS nucleation by Makrides et al. (1978). 
The same two sources also give an analogous expression for the variation of the induction time in the case of heterogeneous (1.e., "seeded" nucleation): it varies in approximate proportion to

$$
N_{1}^{-1 / 3}\left(R_{m d} \rho_{n}\right)^{-1}
$$

where $N_{1}$ is the number of solid particles initially present.

The data of Makrides et al. (1978), Baumann (1959) and Rothbaum and Rohde (1979) clearly exhibit the induction time phenomenon. For some time after a supersaturated MSA solution is prepared, no decrease in MSA is detectable and then, finally, nucleation becomes evident and the MSA concentration drops rapidly. At lower values of $S$ the induction time is longer, consistent with our expectations. Makrides et al. found that at $95^{\circ} \mathrm{C}$ and constant salinity and $\mathrm{pH}$ the induction time varies approximately as

$$
(\ln s)^{-12}
$$

Comparing this expression with (2.18.1), these authors estimated the value of the surface tension to be about $45 \mathrm{ergs} \mathrm{cm}^{-2}$.

The surface tension of the AS-water interface may also be determined from the empirically determined relationship between particle radius and solubility: Using this method, Alexander (1956) found the surface tension to be about 46 ergs $\mathrm{cm}^{-2}$. Iler (1979, p.54) reported values of 54 and 46 ergs $\mathrm{cm}^{-2}$ for colloidal silica sols polymerized at high and low temperatures, respectively. The excellent agreement between the surface tension values determined in these two totally different ways lends strong support to the fundamental correctness of the homogeneous nucleation theory of silica polymerization.

Makrides et al., also found that an increase of $\mathrm{pH}$ by one unit decreased the induction time by about a factor of ten. This is consistent with the concept of hydroxide catalysis and with the form of (2.18.1).

Data presented by Rothbaum and Anderton (1975) show an induction time for the decrease of MSA concentration in Wairakel brines. 
S2.19 The Practical Significance of Nucleation Phenomena and the Study of Homogeneous Nucleation

In the absence of liquid phase nucleation, silica precipitation is limited to molecular deposition on pre-existing solid surfaces. This is a relatively simple and easily described process, and it is slow enough to be of secondary practical importance in most cases, but not all.

Nucleation of colloidal AS in the liquid phase provides the large surface area that makes rapid precipitation possible. The conditions under which nucleation takes place determine just how much surface area it provides. Homogeneous nucleation at large values of $S$ will produce many small particles with a large specific surface area and vice versa.

The threshold S value phenomenon is also of considerable practical importance, because nucleation cannot be prevented by any means above this threshold. (It may, however, be delayed in a kinetic sense by lowering $\mathrm{pH}$ and/or complexIng fluoride by adding aluminum.) To stay below the threshold $\mathrm{s}$ value (under the given conditions) becomes a mafor practical design goal, because, below the threshold, the overall precipitation process will be dominated by the (usually) slower hetexogeneous nucleation mechanism.

The possibility of Induction times on the order of minutes, hours, or even days suggests that flulds with $S$ above the threshold may not precipitate silica within the steam separator units or power plants, but may still precipitate it at some point further downstream instead. This actually seems to be the case at Wairake1 (Rothbaum and Anderton, 1975) and otake (Yanagase et al., 1970). In the case of a really long Induction time (several days) the apparent non-precipitation of silica could conceivably lead to the decision to reinject untreated brine with catastrophic results.

It is clearly of practical interest to be able to predict the kinetics of the homogeneous nucleation process and the number and surface area of the particles produced. The attainment of such a capability requires the formulation of a reasonably rigorous theory of the homogeneous nucleation process in this system, and its reconcillation with extensive high quality experimental data.

In Section 2.17 we discussed the Lothe-Pound Theory of Homogeneous Nucleation and our reasons for choosing to use it. Here we will discuss the substan- 
tial problems that arise in regard to defining and evaluating the contribution of the surface tension to the free energy of the critical nucleus as well.

First, there is no good reason to assume that silica particles of critical nucleus size are nearly spherical in shape. This is a significant point because it affects the relationship between the number of monomer units in the critical nucleus and its area.

Second, the surface tension may vary with surface curvature (1.e., particle size) as well as with temperature and the degree of surface ionization. Because of the extremely small size of the critical nuclei, it may not be possible to rellably extrapolate from the larger particle sizes that are suitable for use in solubility experiments.

Third, a direct determination of the value of surface tension as a function of temperature and degree of surface lonization by the solubility method would probably be impractical. The needed experiments would be fairly tricky. Also, the cructal step in the reduction of particle solubility data to determine surface tension is essentially a numerical differentiation of the experimental data, and is, at best, risky.

We have made a serious attempt to theoretically estimate the value of the surface tension. Our results seem good as such calculations go, but they are not good enough for practical use, given the extreme dependence of the calculated nucleation rate on the value of the surface tension used.

To be of any practical use, the theory needs to be fitted to experimental data. These experiments will be essentially similar to those of Baumann (1959) and Makrides et al. (1978). They will generate a large of amount of homogeneous nucleation data at various temperatures, $\mathrm{pH}$ values, salinities and initial silica concentrations between room temperature and $100^{\circ} \mathrm{C}$. This data will then be fitted using a computer code which models the homogeous nucleation process. The fitting process will basically consist of varying a function which represents the value of the surface tension over the full range of conditions until, an optimal overall fit has been achieved.

A painstaking fit of a rigorous theory to a large amount of high quality experimental data offers the only hope for being able to quantitatively model the homogeneous nucleation process throughout the range of practical interest.

Fitting the surface tension values to the experimental data also has the advantage that it w1ll tend to automatically compensate for possible errors 
and approximations in other parts of the overall model. For example, the possibility of non-spherical critical nuclel need not be dealt with separately, as the correction for this effect will be automatically taken care of In the course of fitting the "surface tension function" to the data.

\section{\$2.20 Other Nucleation Processes}

Two nucleation processes other than homogeneous nucleation of pure AS particles may be expected to occur in some cases. These are:

1) "Heterogeneous nucleation" with particles already present in the brine serving as the heteronuclel. This is not really nucleation, but rather MSA deposition on the preexisting colloldal particles. Successful prediction of the course of this process would require knowledge of MSA deposition kinetics and an adequate characterization of the preexisting solid particles in the brine.

One may also include in this category the case of a brine which comes out of the well already carrying particles of AS or amorphous silicates, as in the case of Niland. Whether or not one wishes to include this case under the rubric of "heterogeneous nucleation" depends mostly on where in the (physical) system one wishes to begin the kinetic modelling exercise.

2) Initial nucleation of an amorphous silicate phase rather than AS 1tself. The presence of other components in the nucleating particles will effect both the bulk free energy and the surface tension.

In practice, the amorphous silicate of fron(II) is likely to be the most important, followed by those of aluminum, magnesium and calcium.

It $1 \mathrm{~s}$ likely that the thermodynamic properties of important amorphous silicate phases will be adequately described sooner or later. In the short term, we expect that the avallable solubility data will provide the most important values. Avallable data on the properties of sillcate melts should also be of use, as it is unlikely that the structure of the precipitated amorphous silicates is very different from the structures of the corresponding melts.

We doubt that surface-tension values for amorphous silicates will ever be properly determined. It would certainly be possible to estimate these values but such estimates would be inadequate for quantitative prediction. 
The extreme conditions under which these phases typically nucleate seem to effectively preclude exhaustive experimental work of the kind proposed above for the case of pure AS.

We suspect that the only case in which a proper treatment of amorphous silicate nucleation will be forthcoming will be that in which the non-silica component is absent from the surface of the critical nucleus, thereby allowing pure silica surface tension values to be used. This, in turn, requires that no more than a few atoms of the other component be present in the nucleus; 1.e., that the particle consists mostly of silica. A well known case of this sort is that of a single ion or a single dissociable molecule serving as a nucleation center for a water droplet. In the AS case, a polymeric aluminum or Iron hydroxide Ion seems most likely to have this effect. If one such entity nucleates a silica particle, its quantitative expression should take the form of a constant negative term in the expression for the free energy of the critical nucleus. The problem with this sort of analysis is that such polymeric species are usually 111-defined and their concentrations unknown.

Above all else, we must know how Important the above processes really are. The cumulative evidence suggesting that homogeneous nucleation is dominant under laboratory conditions seems convincing. The case in the fleld is less clear. To conclustvely answer this question for any specific case w11l require comparing actual fleld experience with the behavior of simulated brines under laboratory conditions. Adequate data appears to be available to do this for the case of Walrakel. A more general answer will require testing possible nucleation-enhancing substances $\left(\mathrm{Al}^{+3}, \mathrm{Fe}^{+3}, \mathrm{Mg}^{+2}, \mathrm{Ca}^{+2}\right.$, clay, etc.) in the laboratory. The execution and qualitative interpretation of such experiments is easy, but their quantitative interpretation probably will not be. Ultimately, one would like to be able to answer such questions by means of theoretical interpretation of brine composition and thermal history data alone.

Fortunately, three factors conspire to make the possibility of heterogeneous nucleation less of an impediment to making meaningful quantitative predictions than it might otherwise be.

F1rst, when conditions favor homogeneous nucleation, it w11l create a truly enormous number of particles (up to about $1 \mathrm{E} 19 \mathrm{dm}^{-3}$ ) and w1ll completely overwhelm the competing heterogeneous nucleation process. 
Second, the kinetics and overall course of the heterogeneous nucleation process are not particularly sensitive to the number of heteronuclei, As we have noted in 52.18 , the induction time for heterogeneous nucleation varies only as the minus one third power of this number.

Third, aluminum and magnesium, which are probably the most effective elements in regard to inducing heteronucleation by amorphous silicates, are rarely present in geothermal brines in detectable amounts precisely because of the extreme stability of their compounds with sllica. (Significant amounts of Iron are, however, often present in high salinity brines because of the solubilizing effect of iron-chloride complex formation.)

52.21

Colloidal Stability and Coagulation of Colloidal Amorphous Silica

The classical (Landau-Derjagin-Verwey-Overbeek) theory of colloidal stability holds that a suspension becomes unstable when attractive dispersive (Van der Waals) forces exceed repulsive electrostatic forces. In practice, the balance between the two forces is determined by the strength of the electrostatic force, which is strongly effected by the chemical environment and solvent properties while the dispersive force is not. The most basic determinant of the electrostatic force is the magnitude of the surface charge on the colloidal particles. This is determined by ion adsorption and the dissociation of surface groups. The intensity of the electric field near each particle is determined by the degree of shielding by oppositely charged lons in the surrounding medium. In general, increasing electrolyte concentration In the medium decreases colloldal stability, and a greater electrolyte concentration 16 needed to coagulate a collo1d with a greater surface charge. (Al1 this is set forth in the classic book by Verwey and Overbeek, 1947). Unfortunately, this "classic" theory does not work in the case of col101dal AS (Allen and Mat1jević, 1969, 1970, 1971; Iler, 1975a). Allén and Matijevic found that in most cases a smaller electrolyte concentration was needed to coagulate colloldal AS at higher $\mathrm{pH}$, despite the fact that higher pH corresponds to a greater surface charge and, thereby, a greater electrostatic repulsive force. Furthermore, they found that the concentration of any given electrolyte needed to coagulate the silica within an hour was determined 
by the cation's ability to exchange for protons on the AS surface. For any electrolyte at a given $\mathrm{pH}$, the "critical coagulation concentration" (c.c.c.)* was just that at which the number of protons released from the AS surface per unit area equaled a certain value which was a function of $\mathrm{pH}$ alone. This "critical exchange curve" (c.e.c.) was found to be common to all monovalent cations studied throughout the $\mathrm{pH}$ range studied. The "critical exchange values" for calcium and trivalent lanthanum also fall on the curve up to about $\mathrm{pH}$ 8. At higher pH's the "critical exchange" values for calcium fell below the curve for monovalent cations.

Later, Ailen and Matijević (1971) directly determined the amount of calcium ion which was actually adsorbed on the AS surface at the c.c.c., as contrasted to the number of protons released by calcium sorption. (This distinction is necessary in the case of di- and higher valent cations, because these may, on the average, exchange for fewer protons than are necessary to balance their charge). They found that a plot of equivalents of calcium adsorbed at the c.c.c. versus $\mathrm{pH}$ fell on the "critical exchange curve" up to about $\mathrm{pH}$ 9. The calcium points again fell below the c.e.c.

* In the usage of these authors, the c.c.c. Is approximately that concentration at which coagulation-related light scattering becomes just detectable one hour after mixing. One hour was chosen as the time of measurement because it was found to be the "critical time" for silica coagulation (Allen and Matijević, 1969)

The concept of the "critical time" was introduced by Težak et al. (1951). Basically, it is the length of time within which the most important mechanism of electrolyte-induced flocculation will become apparent if the electrolyte concentration is high enough for it to be effective. The c.c.c. Is the corresponding threshold concentration as determined at the critical time. Other, slower processes which cause an increase in light scattering may manifest themselves at concentations below the c.c.c. after some period of time longer than the "critical". Težak et al. suggest that these processes usually involve "recrystall1zzation" of the colloidal material into larger particles (i.e., colloid aging). 
above pH 9, but became obviously erratic as a consequence of experimental difficulties at still higher pH. These results suggest that the c.e.c. may actually be common for all cations, regardless of charge, if it is interpreted as being the curve of "critical cation adsorption".

The graph of the critical exchange curve presented by Matijevic (1973) is fitted reasonably well by the empirical formula:

$$
\log \mathrm{E}=-2.34+0.210 \mathrm{pH}
$$

where $E$ is the density of sorbed cationic charges expressed as a fraction of the total number of lonizable silanol groups on the As surface. (This is about $3.9 \mathrm{E} 14 \mathrm{~cm}^{-2}$, according to Allen and Matijevid, 1970). This formula applies to a suspension containing $1 \mathrm{~g} \mathrm{~L}^{-1}$ of Ludox HS colloidal silica with a specific area of $200 \mathrm{~m}^{2} \mathrm{~g}^{-1}$. (This and all further data quoted from these authors are from experiments performed at room temperature.)

The logarithm of the c.c.c. for NaCl and $0.2, \mathrm{~L}^{-1}$ of Ludox HS is fitted well by the formula:

$$
10 \mathrm{~g} \text { c.c.c. }=2.201-0.2668 \mathrm{pH}
$$

The analogous formula for $\mathrm{KCl}$ is:

$$
\log c . c . c .=1.883-0.2266 \mathrm{pH}
$$

Both of the above formulas are our own fits to graphical data presented by Allen and Matijevic (1970).

Other data (Allen and Matijevic, 1969) suggest that the c.c.c. for $\mathrm{Na}^{+}$ Is Independent of the Identity of the counterion (anion) and of the concentration of colloldal silica over the range 0.2 to $2 \mathrm{~g} \mathrm{~L}^{-1}$. The threshold coagulating concentrations for $\mathrm{NaCl}$ as determined 24 hours after mixing were uniformly lower by about a factor of two than were the one hour c.c.c. values. It is unclear whether or not this slower coagulation at lower concentration was caused by the same mechanism as the more rapid coagulation detected after one hour.

Iler (1975a) studied the relationship between $\mathrm{pH}$, particle size, and the c.c.c. for calcium. His experiments were performed at $\mathrm{pH} 8.1,8.75$ and 9.5 
using particles of specific area ranging from 21 to $540 \mathrm{~m}^{2} \mathrm{~g}^{-1}$. His results for the c.c.c. (In moles $\mathrm{L}^{-1}$ and determined at one hour) are well fitted by the relationship

$$
c . c . c .=0.03885-0.004 \mathrm{pH}+7.2 \mathrm{E} 10 \exp (-3.326 \mathrm{pH}) \mathrm{A}
$$

where $A$ is the specific surface area of the colloidal AS in $\mathrm{m}^{2} \mathrm{~g}^{-1}$. Allen and Matijevil's (1969) data for the c.c.c. of calcium is also well described by this relationship in the $\mathrm{pH}$ range 8.2 to 9.5 . However, the above formula gives values somewhat below those found by Allen and Matijevic for colloidal AS with $A=200$ in the $\mathrm{pH}$ range 7.2 and 8.0 . The discrepancy is about a factor of two at $\mathrm{pH} 7.2$.

Iler also separately determined the number of protons released and the $n$. ber of calcium lons adsorbed at the c.c.c. He found that, to within experimental error, these quantities were independent of particle size. Both quantities increased moderately with increasing pH. The ratio of protons released to calcium Ions adsorbed was found to be 1.6 at $\mathrm{pH} 8.1,1.25$ at $\mathrm{pH} 8.75$ and 1.05 at $\mathrm{pH}$ 9.5. These results demonstrate that the "critical sorption curve" is actually independent of particle size; the variation of c.c.c. with particle size is actually caused by the fact that smaller particles adsorb calcium ions less strongly than do larger ones.

Unfortunately, no one seems to have studied the relationship between c.c.c. for sodium and particle size. However, Heston et al. (1959) and Iler (1975a) found that the titration curves of colloidal AS in NaCl solutions were independent of particle size. Combining this observation with the apparently universal nature of the "critical sorption curve" leads to the prediction that the c.c.c. for sodium should also be independent of particle size. The difference between sodium and calcium is obviously due to the charge difference. More specifically, we believe that it is due to the fact that, on the average, a calcium ion displaces less than two protons on the AS surface when it is adsorbed.

It is apparent from Eq. $(2.21 .4)$ that, at $\mathrm{pH} 8.1$, the dependence of the c.c.c. of calcium on particle size is powerful indeed. The value of the calctum c.c.c. changes by more than an order of magnitude between $A=0$ 
(extrapolated) and $A=540$. This has the practical implication that AS particles in a calcium-rich geothermal brine will tend to coagulate out after they have grown to a certain size. Likewise, aggregates of several particles formed by whatever means will tend to coagulate out more rapidly than single particles. Finally, monodisperse particles will stick to flat AS surfaces much more readily than to each other. Indeed, it seems possible that there are conditions under which the rate of particle removal by deposition on flat surfaces will exceed the rate of removal by formation and growth of suspended aggregates in the liquid phase.

Matijevic (1973) has reported the destabilizing effects of sodium and potassium lons to be additive. This is to be expected from the universal nature of the critical adsorption curve and from the likelihood that at the relatively low sorption densities of greatest interest each of the cations is adsorbed from mixed electrolyte solutions more or less independently of the others. Thts observation much simplifies the prediction of the destabilizing effects of mixtures of sodium, potassium and calcium. Given the concentration of each and the $\mathrm{pH}$, one merely needs to compute the fraction of the corresponding c.c.c. that each concentration represents. If these add up to unity or greater, the collotd is unstable.

\section{S2.22 The Mechanism of Destabilization}

Both Allen and Matijevil and Iler came to the obvious and well substantiated conclusion that surface fon exchange destabllizes the colloid. Allen and Matijevil suggest that this may be due to the loss of hydrogen bonding ability of the unionized silanols. We doubt that this is a significant factor, because the highly polar lonized silanol-cation pairs at the ion exchanged sites should be at least as strongly hydrated as the original silanols. Iler suggests that the destabilization is electrostatic in origin: ion exchange creates a mosalc of negative and positive charges on the particle surface. When two such surfaces are properly juxtaposed, the opposite charges attact each other. The constancy of the "critical sorption curve" is easily understood in terms of this model: at any given $\mathrm{pH}$ and exchange value, the net charge of the particle and the total positive charge within the mosalc are the same, regardless of the identity of the cation exchanged. 
An important fact which Allen and Matijević failed to comment on is that the constancy of the critical exchange curve also holds over large variations In lonic strength. This must be so because a given exchange value usually corresponds to considerably different concentrations and lonic strengths as one goes from electrolyte to electrolyte at a given $\mathrm{pH}$. This means that both the attractive and the repulsive forces between the particles must be affected the same way by electrostatic shielding. This is inconsistent with the "dehydration" theory of destabilization, as well as with coagulation by Van der Waals type attractive forces. (It is precisely the fact that van der Waals forces are unaffected by electrostatic shielding while electrostatic repulsive forces are that causes "classical" collolds to be destabilized by electrolytes).

In an electrolyte solution, the interaction of charged particles is described by the shielded Coulomb potential. The repulsive force between particles of like charge is simply due to the fact that the overall charges of both particles are nonvanishing and of the same sign. Therefore, the repulsive force may be said to arise from the interaction of the monopole terms in the multipole expansions of the electric potentials of the two particles. The attractive forces between two AS particles with adsorbed cations on their surfaces are due to monopole-dipole and higher order interaction terms.

In the case of a spherical particle at the limit of low lonic strength, the monopole term in the one particle potential is

$$
\psi=Q(R \varepsilon)^{-1} \exp ((r-R) / d) /(1+r / d)
$$

where

$$
\begin{aligned}
& r=\text { the particle radius } \\
& R=\text { the distance from the center of the particle } \\
& d=\text { the thickness of the diffuse double layer } \\
& \varepsilon=\text { the dielectric constant of the solvent. }
\end{aligned}
$$

The major difference between (2.22.1) and the corresponding expression for the unshielded Coulomb potential is the presence of the exponential term which makes the value of the potential decay more rapidly with increasing $R$. The electrostatic shielding factor enters into the corresponding expressions for the dipole and higher terms of the potential in the same way. Therefore, 
varying the fonic strength changes the values of all of the various interaction terms by approximately the same factor, and the balance between the attractive and repulsive forces is approximately unaffected by variations in the ionic strength as long as the charge distribution on the particle surfaces stays about the same.

The only avallable study of the kinetics of flocculation of colloidal AS seems to be that of Hahn and Stumm (1968). They followed the early stages of coagulation by aluminum salts of $205 \mathrm{~m}^{2} \mathrm{~g}^{-1}$ colloidal As using the light scattering technique. The initial rate of decrease of the total number of particles and aggretates was found to be proportional to the square of the total number

$$
\mathrm{dN} / \mathrm{dt}=-\mathrm{k} \mathrm{N}^{2}
$$

where $k$ is an empirically determined rate constant. This is consistent with a binary collision model of the early part of the coagulation process. In the case of particles large enough for the collision rate to be determined by turbulent motion, $k$ was found to have the expected Iinear dependence on stirring rate. It was also estimated that only on the order of 1 in 100 Interparticle collisions resulted in adhesion. Little more can be said on the basis of the avaflable data.

All work on the coagulation of colloldal As that we found reference to was at room temperature. Therefore, the effect of temperature is unknown, and badly needs to be studied.

\section{S2.23 Ion Exchange on the Surface of Amorphous Sillca}

As discussed In 52.13 , the rate of molecular deposition of dissolved silica on the surface of solid AS Is proportional to the surface density of dissociated silanol groups. Our own work has demonstrated that the surface tension of the AS-water Interface and, thereby, the rate of homogeneous nucleation of colloidal AS particles ts strongly influenced by the surface density of lonized silanol groups under the given conditions as well. As discussed in $\mathrm{S} 2.21$ and $\mathrm{S} 2.22$, the $\mathrm{pH}$ and surface density of adsorbed cations determine the stability of colloldal AS. All in all, we see that the surface 
Ion exchange properties of AS determine many of its chemical properties. The ability to predict the electrostatic state of the AS surface under any given conditions would contribute mightily to predicting many of the chemical and colloldal properties of AS.

The exchange of sodium on the surface of AS at room temperature has been extensively studied. The most extensive and useful studies are those of Bolt (1957), Heston, Iler and Sears (1960), and Allen, Matijević and Meites (1970). The latter three authors fitted their data using a more-or-less rigorous physical model which is based on the assumption that all dissociated silanols have cations bound to them. (This is approximately true at higher salt concentrations, but is a rather poor approximation at low salinities.) We used a slightly more general version of this model which accounts for the presence of "unpaired" ionized silanols in an approximate way in our own work.

There is also a smattering of information about the adsorption of other cations on the surface of AS available in the literature. See, for example, various papers in the Bibliography of this report (in particular, those papers cited in the preceeding two Sections), the review of this general subject in Iler's book (1979, pp. 659-76 and elsewhere), and the review by wiese et al. (1976). L.H. Allen's (1970) thesis also contains titration data for a variety of salt media that are not fully presented elsewhere.

Unfortunately, the data available in the 11terature is almost completely limited to room temperature conditions. (The paper by Dugger et al., 1964, is the only important exception to this.) A study of ion exchange at higher temperatures is the most pressing experimental need in this area. A study of the exchange properties of calclum as extensive as those of sodium cited above would also be highly desirable.

Fortunately, what appears to be an adequate theoretical formalism for describing the exchange of cations for protons on the surface of AS (as well as those of other amphoteric and acidic oxides) is available.. This is the site binding model of Yates, Levine, and Healy (1973) in the form used by Yates, James and Leckie (1979). (For a detalled review of the early development of this model and related topics see wiese et al., 1976.) This model is fundamentally similar to that used by Allen, Matijevif and Meites (1971), but is an improvement on the latter in that it correctly accounts for the fact that not all dissociated surface silanols have cations bound to them. 
The outstanding theoretical need in this area is to extend the site binding model to make it applicable to small spherical particles as well as to flat surfaces. This should not be very hard to do.

\section{S2.24 The Gelation of Collotdal S111ca}

Concentrated slifica sols (several percent $\mathrm{S}_{2}$ or more), form a gel phase under certain conditions. Gel formation does not ordinarily involve the separation of silica from water. Rather, a homogeneous sol turns into a homogeneous gel. The dilute sols of geothermal interest (less than $1 \mathrm{~g} \mathrm{~L}^{-1}$ $\mathrm{SiO}_{2}$ ) do not form gels because they do not contain enough silica particles to "fill", the whole volume of the sol with a more-or-less continuous network. The gelation process is still of interest, however, because the processes involved in gelation at high sol concentrations should cause an analogous type of flocculation or coagulation at lower sol concentrations under the same conditions.

The only available quantitative data on the gelation of well-defined silica sols seems to be that provided by the duPont Company in their commerclal sllica sol product literature (duPont, no date). Iler (1973) also discusses gelation in much greater detail but less quantitatively.

The main facts regarding the gelation of dense $\left(10\right.$ to $\left.30 \% \quad \mathrm{S1O}_{2}\right)$ sols are:

1) The gel time may be anywhere from a few minutes to lmmeasurably long, depending on concentration, particle size and other factors.

2) All else constant, there is a certain $\mathrm{pH}$ value at which gelation is most rapid. This $\mathrm{pH}$ value is generally near 5 , but may be anywhere from about 4 to about 7 depending on other factors.

3) Increasing electrolyte concentration decreases gel time and shifts the $\mathrm{pH}$ corresponding to minimum gel time to higher values.

4) Increasing temperature reduces gel time greatly. In the case of $10 \mathrm{w} \%$ sols of Ludox HS at $\mathrm{pH} 5$, the apparent activation energy is about $18.3 \mathrm{kcal}$ (from dupont product data).

5) Gel time decreases with Increasing sol concentration. For Ludox HS at $\mathrm{pH} 5$ and $98.9^{\circ} \mathrm{C}$, the gel time is inversely proportional to the square of the concentration between 10 and $30 \mathrm{w}$. At lower temperatures the concentration dependence is even greater (Ibid.). 
6) At a given concentration, gel time decreases rapidly with decreasing particle size. Iler (1973, p.43) puts forth this approximate kinetic law:

"The rate of gelling appears to be proportional to the total area of silica surface present in a given volume of sol". (Note that this contradicts the conclusion under 5) above. However, it does give a feeling for the relative effects of varying concentration and particle size.)

The apparent activation energy for gelation appears to be approximately the same as that for the formation of a siloxane bond. It is likely that the rate-determining step in the gelation process is the formation of siloxane bonds between two particles which are in contact. The increase of gel time with decreasing $\mathrm{pH}$ below a certain value is a consequence of the well-known $\mathrm{pH}$ dependence of the rate of siloxane bond formation.

The increase in gel time with increasing $\mathrm{pH}$ at higher $\mathrm{pH}^{\text {'s }}$ is clearly due to increasing negative charge of the particles. This behavior is consistent with the classical theory of colloid stability. It is in qualitative disagreement with the cation sorption destabilization model discussed in S2.22 This suggests that the interparticle attraction caused by cation sorption is not a significant factor in the gelation process. It seems 11kely that some combination of van der Waals forces, hydrogen bonding, and hydrophobic attraction holds the colliding particles together long enough for the formation of interparticle siloxane bonds.

We conclude that there are also relatively weak "classical" attractive forces which mediate gelation under conditions of negligible cation sorption. Overall, it appears that these forces are too weak and the gelation process too slow to be of much practical importance under most geothermal brine conditions. It is clear that under conditions of rapid coagulation that is induced by cation adsorption one need not concern oneself about the parallel "classical gelation" mechanism because of its very low rate at the low sol concentrations of greatest interest to geothermal chemistry. 
CHAPTER THREE - THE KINETICS OF SILICA POLYMERIZATION IN AQUEOUS SOLUTION

\section{S3.1 Introduction}

Silica usually precipitates from geothermal brines as amorphous silica or amorphous silicates. This conclusion is supported by geothermal field experience, laboratory research and theoretical considerations. The scope of this study was restricted accordingly.

The process of amorphous silica precipitation from supersaturated bulk aqueous phase consists of the following steps:

1) Formation of silica polymers of less than critical nucleus size.

2) Nucleation of an amorphous silica phase (from here on simply AS) in the form of colloidal particles.

3) Growth of the supercritical AS particles by further chemical deposition of silicic acid on their surfaces.

4) Coagulation or flocculation of colloidal particles to give a gel.

5) Cementation of the particles in the gel by chemical bonding and further deposition of silica between the particles.

6) Rarely, growth of a secondary phase in the interstices between the AS particles.

Step 6) is known to occur in the high temperature brine lines and separators at the Niland test facility. The secondary phase observed there is largely Iron sulfide, Fes (Austin et al., 1976, pp. 58-62). What appears to be secondary growth of calcite in silica scale has been reported in an evaporative cooling system supplied with silica and calclum-rich water (Midkiff and Foyt, 1976 and 1977).

When a solid surface is present, a layer of amorphous silica forms on 1t, and further deposition may proceed as step 3) alone. If an AS collold is present in the medium, the particles may adhere to the surface in analogy to steps 4 ) and 5 ), and step 6 ) may follow.

Throughout this report we employ the term molecular deposition to signify the deposition of dissolved silica on surfaces by means of step 3 ) alone. This special term is introduced to avold possible confusion with deposition of silica in colloidal form which would be analogous to step 4).

The important role of the homogeneous nucleation step in silica pre- 
cipitation from solution was recently recognized by A.C. Makrides and his coworkers at the EIC Corporation of Newton, Mass. (Makrides et al., 1978).

Past ignorance of this crucial fact has caused much confusion and misinterpretation in the silica literature. The problem is that steps 1), 2) and 3) are qualitatively different, and follow different rate laws. This makes it nearly worthless to try to fit the kinetics of the whole process to one kinetic equation as has usually been the case in the past. This pitfall has been avoided in the work reported here.

The kinetics and phenomenology of steps 2) and 3) have been studled by us in great detail, both separately and together. Step 1) happens too rapidly to have any effect on the overall kinetics. Steps 4) and 5) have been studied in somewhat less detall in work reported elsewhere (Weres et al., 1980).

\section{S3.2 Experimental Methods}

The basic experimental design and methods are outlined here. Further details of experimental technique are presented in Appendix 3.2. All in all, our experimental techniques most closely resemble those of Baumann (1959) and Makrides et al. (1978).

Approximately 300 experimental runs were performed, and about two-thirds of these produced useable data.

The basic experimental technique employed was to prepare a buffered solution of given $\mathrm{pH}$, molybdate active silica concentration (henceforth abbreviated as MAS), salinity, etc., and keep it at a constant temperature for some length of time while periodically withdrawing small samples to analyze for remaining MAS. The molybdate yellow method was used to determine the MAS concentration as the reaction progressed.

The kinetic experiments fell into two broad categories:

1) experiments of the molecular deposition type, in which colloidal silica was added to the solution at the beginning of the experiment and served as a solid substrate for molecular deposition, and

2) experiments of the homogeneous nucleation type, in which no colloidal sllica was added, but, instead, it formed by means of homogeneous nucleation as the reaction progressed. 
Timing of the Experiments. The great bulk of our experiments were planned to have a duration of about 60 to 90 minutes, and most of these were done three at a time with manual sampling at intervals of 5 or 10 minutes. Aside from greatly increasing productivity, the three-at-a-time approach best allowed the effects of single variables ( $\mathrm{pH}$, salt content, etc.) to be 1solated with least Interference by random errors, etc. Because pH has a large and more-or-1ess easlly predictable effect upon reaction rate, we were able to devise most of our experiments to fit into this convenient time frame.

Those experiments which could not be fitted into 90 minutes because of desired low $\mathrm{pH}$ or low initial MAS concentration were run as long as required. Most of the experiments dealing with fluoride catalysis required 4 to 6 hours, and some, up to 12 hours. (Here we were able to exercise some degree of control by varying the initial silica concentration:) Nucleation experiments with Initial silica concentrations below about $0.6 \mathrm{~g} \mathrm{SiO}_{2} \mathrm{~L}^{-1}$ required up to 100 hours to complete. In this case samples were taken morning and afternoon from Monday morning to Friday afternoon.

Temperature. Experiments were performed at $50,75,30,100$ and $23^{\circ} \mathrm{C}$, with the number of experiments performed at each temperature decreasing in about that order. The temperature in our laboratory is steady at $23^{\circ} \mathrm{C}$, and so, when working at that temperature, we simply let the reaction vessels stand on the benchtop.

Experiments at $100^{\circ} \mathrm{C}$ were run in a heater-mantled, three-necked flask equipped with a reflux column. At $100^{\circ} \mathrm{C}$, the decline in MAs concentration was monttored in a continuous flow mode employing a peristaltic pump and mixing manifolds (Fig. 3.1). This system is discussed in detall in Appendix 3.2.

At temperatures below $100^{\circ} \mathrm{C}$, the usual method was to take discrete 1-ml samples with a Becton-Dickinson ground glass syringe at intervals of five or ten minutes. This resulted in more point scatter than the continuous flow technique, but eliminated chronic difficulties with pump servicing and stability, and allowed several (typically three) experimental runs to be made at the same time. 
CONTINUOUS FLOW KINETIC SYSTEM FOR EXPERIMENTS AT $100^{\circ} \mathrm{C}$

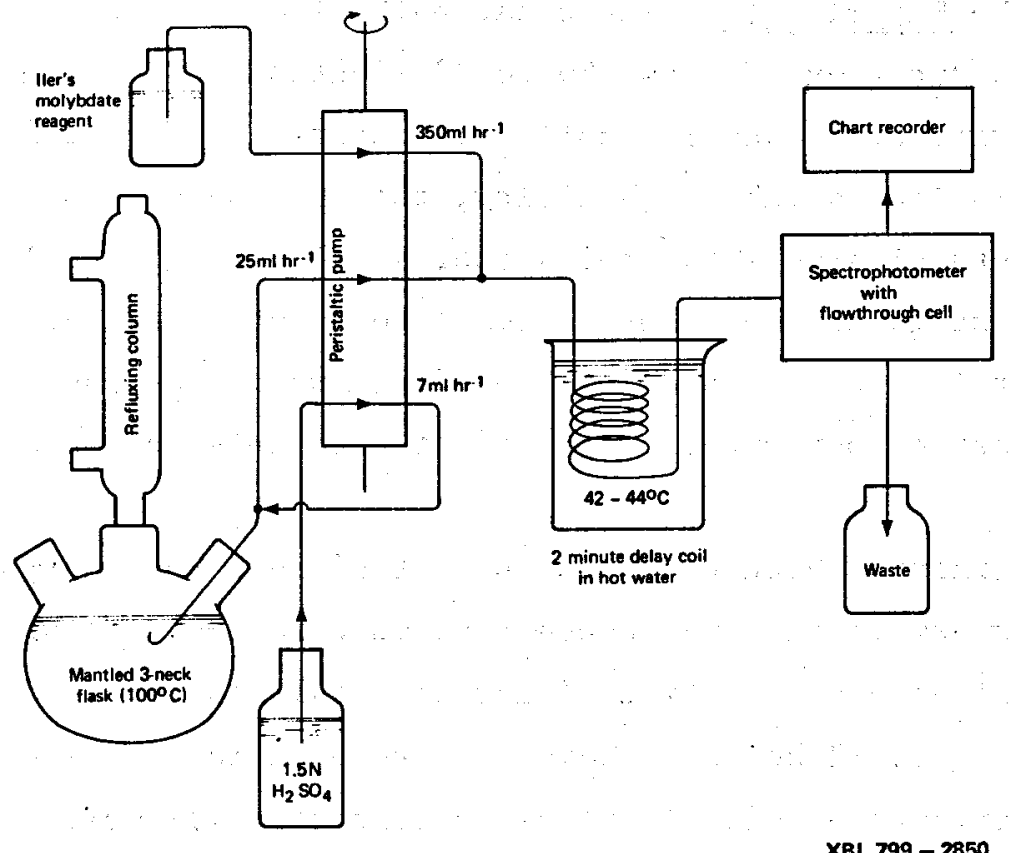

Fig. 3.1. This system is discussed in detail in Appendix 3.2.

At temperatures other than 23 and $100^{\circ} \mathrm{C}$, the solutions were kept in a thermostatted water bath fitted with a plexiglass cover. Spot checks indicated a typical range of temperature fluctuation of $\pm 1{ }^{\circ} \mathrm{C}$ at temperatures below $100^{\circ} \mathrm{C}$, and essentially constant temperature at $100^{\circ}$.

Silica stock solution. A solution containing about $2.0 \mathrm{~g} \mathrm{~L}^{-1} \mathrm{SiO}_{2}$ as sodium metasilicate was used as the dissolved silica stock solution. The absence of a significant concentration of oligomeric species in this stock solution was indicated by the fact that a few experiments performed using stock solutions with a $\mathrm{Na}: \mathrm{SiO}_{2}$ ratio twice as high gave identical results.

This stock solution was prepared as follows: dissolve $9.45 \mathrm{~g}$ reagent grade sodium metasilicate, $\mathrm{Na}_{2} \mathrm{~S}_{3} \mathrm{O}_{3} \cdot 9 \mathrm{H}_{2} \mathrm{O}$ in one liter of doubly delonized water. Standardize before use.

Buffers. Different buffers were used in different $\mathrm{pH}$ ranges. Below $\mathrm{pH}$ 2.5, a maleate-citrate mixture was used. Between $\mathrm{pH} 2.5$ and 6 , citrate alone was used. Between about $\mathrm{pH} 6$ and 8.5 (most of our work), a mixture of meleate and barbital (5,5 L diethylbarbituric acid; also known as Veronal) was used. Above $\mathrm{pH} 8.5$, a mixture of barbital and glycine was used. 
In the case of the barbital-maleate and barbital-glycine systems, the buffer stock solutions were prepared from the sodium salts of the buffering compounds. Each compound present in these stock solutions was at a concentration of $0.1 \mathrm{M}$. The c1trate-maleate and c1trate buffer stock solutions were prepared in acid form, with each acid present in $0.1 \mathrm{M}$ concentration. In all cases, the buffer stock solution was diluted five-fold in preparing the actual experimental solutions. Therefore, the buffering compounds were each present in $0.02 \mathrm{M}$ concentration in the experimental solutions.

The barbital used was Mallinckrodt "Barbital: Purffled Powder" Intended for pharmaceutical use.

The barbital-maleate buffer stock solution was prepared as follows: dissolve 18.4 grams barbital and 11.6 grams malelc ac1d (J. J. Bates) in 1 liter $0.1 \mathrm{~N} \mathrm{NaOH}$.

Colloldal silica. The colloldal silica employed in the molecular deposition experiments was duPont Ludox. Ludox is a technical grade product, but is rather pure. It was supplied to us free of charge by the duPont Company.

We used Ludox varieties TM, HS, and SM, and determined their specific surface areas by means of the Sears:(1956) titration method. The spectfic surface areas determined for the our samples of TM, HS, and SM were 157, 242 and $359 \mathrm{~m}^{2} \mathrm{~g}^{-1} \mathrm{SIO}_{2}$, respectively. These values correspond to initial particle radil of about $8.7,5.6$ and $3: 8$ nanometers.

The colloidal silica stock solutions were prepared as follows: filter concentrated Ludox TM, SM, \& HS sols obtalned from manufacturer through fluted filter paper in large funnel, determine concentration of $\mathrm{SiO}_{2}$, and dilute with delonized water to $100 \mathrm{~g} \mathrm{SiO}_{2} \mathrm{~L}^{-1}$.

other materlals and water. All chemicals used other than Ludox and barbital were of reagent grade.

All solutions were prepared using twice doubly delonized water. The water was delonized using mixed anion and cation exchange resins. The water coming out of our laboratory's defonlzed water tap was already once doubly delonized in this way. It was doubly delonized a second time using our own column before using 1t. Blank determinations using this water showed not enough silica (or phosphate) to give a detectable color with the molybdate yellow method. 
Our data showed that the only potential contaminants which may influence the chemistry of silica in trace concentrations are fluoride and aluminum. (Fluoride is a significant catalyst below about $\mathrm{pH} 5$, and aluminum counteracts its effect. Aluminum is itself a potent reaction inhibitor above pH 7.5. See S3.6 and S3.15) However, none of our data showed what would have been the obvious effects of contamination by elther. Also, none of the experiments in the low $\mathrm{pH}$ range in which fluoride contamination might have been significant employed either Ludox or barbital which would have been the likeliest sources of contamination. Colorimetric analysis of the barbital gave an upper limit value of $0.1 \mathrm{ppm}$ for possible contamination of the experimental solutions with aluminum from this source. We believe that any effect caused by this little aluminum would be smaller than that of experimental error.

Preparation of the Experimental Solutions. The reactions were initiated by rapidly mixing two preheated solutions, one acid and one alkaline. In all cases, the alkaline solution was simply the appropriate amount of the sodium metasilicate stock solution.

When the barbital-maleate or barbital-glycine buffer system was used, the acid solution contained the buffering compounds, the added salts (if any), and the amount of $0.3 \mathrm{~N} \mathrm{H}_{2} \mathrm{SO}_{4}$ needed to neutralize the silica stock solution and adjust the buffer to the desired $\mathrm{pH}$. The the first steps in compounding the acid solution were to $\mathrm{mix}$ the appropriate amount of buffer stock solution and the added salts (in solid form, if any) with some water, and then to adjust the $\mathrm{pH}$ of this mixture to the desired final value by titrating it with $0.3 \mathrm{~N} \mathrm{H}_{2} \mathrm{SO}_{4}$. When no salts were added, all this was done at room temperature. If salts were added, the mixture was heated to about 50 or $60^{\circ} \mathrm{C}$ in order to keep the barbital in solution. (The temperature at which this titration is performed is not critical, because the $\mathrm{pH}$ of barbital-maleate buffer varies little with temperature.) After this titration procedure, the amount of $0.3 \mathrm{~N} \mathrm{H}_{2} \mathrm{SO}_{4}$ needed to neutralize the silica stock solution was added, as well as the amount of water needed to bring the final solution volume up to that desired.

Typically, the total concentration of sodium ion introduced into the experimental solution by way of the silica and buffer stock solutions was about $0.09 \mathrm{M}$. 
the higher concentration $Y_{\text {emp }}$ values. In other words, the low $c_{1}$ curves drop too quiclily relative to the higher $c_{1}$ curves to be fitted by the same formula for $\gamma$. This deviation is well accounted for by the hypothesis that heterogeneous nucleation is the dominant nucleation mechanism at the lowest $c_{i}$ value at each temperature.

Because our model considers only homogeneous nucleation, the data that was effected by heterogeneous nucleation had to be dropped from the fitting process. It was found that deleting the four hardest to fit curves markedly improved the overall fit of the remaining fourteen, while dropping additional ones caused little further improvement. These four were: $c_{i}=0.4$ and 0.5 $\mathrm{g} \mathrm{L}^{-1}$ at $50^{\circ} \mathrm{C}, c_{i}=0.5 \mathrm{~g} \mathrm{~L}-1$ at $75^{\circ} \mathrm{C}$, and $c_{1}=0.75 \mathrm{~g} \mathrm{~L}-1$ at $100^{\circ} \mathrm{C}$. The remaining fourteen curves were best fitted with these values:

$$
\begin{aligned}
& \mathrm{H}_{Y}=63.68 \mathrm{ergs} \mathrm{cm}-2 \\
& S_{Y_{Y}}=0.049 \mathrm{ergs} \mathrm{cm} \mathrm{K}^{-1} \\
& \mathrm{n}_{0}=6.84 \mathrm{E} 14 \mathrm{~cm}^{-2} .
\end{aligned}
$$

With these values, the RMS deviation between the values of. $Y_{\text {emp }}$ and $\gamma_{f i t}$ (for the fourteen values that were fltted) was $0.7 \mathrm{ergs} \mathrm{cm}^{-2}$, and the residual RMs "error in $\log \tau$ " was $0.110 \mathrm{l}$ units.

A comparison of experimental and calculated curves (using $\gamma_{f i t}$ for $\gamma$ in all cases) for $50^{\circ} \mathrm{C}$ is presented in figure 3.18. The disagreement between theory and experiment at $c_{1}=0.5 \mathrm{~g} \mathrm{t}^{-1}$ is due to the effect of heterogeneous nucleation. The excellent ag reement in the other four cases is a gratifying confirmation of the fundamental validity of the theoret lcal model that was used. (Note, however, that the agreement of theory with experiment is not quite as good at the other two temperatures. See Table 3.1.)

For $c_{1}=0.6$, two curves are presented in FIg. 3.18. One of them was calculated using the "multistate kinetlcs" formalism, and the other one, using the approximate expression $(3.8 .4)$. The former seems to have a slightly "better shape", but this modest improvement hardly seemed to justify further bother with the cumbersome "multistate kinetics" algorithm, and it was declded to delete it from the code.

Table 3.1 also contains some theoretically calculated results. Particularly noteworthy is how small some of the values of $n^{*}$ are. Model building "experiments" reveal that AS particles in this size range are not even close to being spherical in shape. Nonetheles,, the theory used here, which assumes 
When the citrate or citrate-maleate buffer system was used, the preparation of the acid solution involved doing a test titration first. The test solution was prepared by mixing the desired amounts of silica stock solution, buffer stock solution (in acid form), and added salts, if any (in solid form). This mixture was titrated with either $0.3 \mathrm{~N} \mathrm{H}_{2} \mathrm{SO}_{4}$ or $1.0 \mathrm{~N} \mathrm{NaOH}$ to the desired $\mathrm{pH}$ value, and the volume of acid or base solution required was recorded. The acid starting solution actually used was then formulated by mixing the appropriate amounts of buffer stock solution and added salts, the amount of acid or base needed to obtain the desired final $\mathrm{pH}$ as determined above, and the amount of water needed to give the desired final volume. This procedure typically introduced about $0.05 \mathrm{M}$ of sodium derived from the silica stock solution into the experimental solution, and a smaller amount from the $\mathrm{NaOH}$ used to adjust the $\mathrm{pH}$ (if any).

When Ludox was employed, it was added last, after the acid and alkaline solutions had been mixed and a $1 \mathrm{ml}$ allquot withdrawn for the purpose of establishing an inftial silica concentration.

Concentration units employed. Al1 liquid measuring was done at or near room temperature, and all concentrations were calculated on a per liter at room temperature basis. For example, we formulated a solution to contain, say $1 \mathrm{~g}$ of $\mathrm{SiO}_{2}$ and 1 mole of $\mathrm{NaCl}$ per liter at room temperature, regardless of what temperature we actualiy intended to use in the experiment. Thus, the nominal per liter concentrations were about equal to the actual ones in the case of work at 23 and $30^{\circ} \mathrm{C}$, but not at the higher temperatures. When no salts were added, the nominal per liter concentrations were approximately equal to concentrations per kilogram of water (i.e., molal concentrations) at all temperatures. When salts were added, the nominal per liter concentrations, were not equal to molal concentrations at any temperature, but they were independent of the actual temperature of the experiment. Therefore, during data analysis, we could always convert our concentration values to molal values by using a correction factor which depended only on the concentration of the added salt and the tabulated values of its partial molal volume as determined at room temperature.

Throughout this report, concentrations expressed in terms of grams or moles per 1iter are "nominal" concentrations referred to room temperature as discussed above; 1.e., l M NaCl means that that solution have would been 
$1 \mathrm{M}$ In NaCl if analyzed at room temperature, but actually was not at the temperature of the experiment.

Al1 silica analyses were made using the molybdate yellow method and, therefore, all of the concentrations determined were concentrations of molybdate-active silica. This is not quite the same as "monomeric silica": or as monosilicic acid (henceforth abbreviated as: MSA), because silicic acid dimers and trimers are molybdate-active as we11. In practice, there was no way we could distinguish between MAS and monomeric silica (which includes MSA and the ion $\mathrm{H}_{3} \mathrm{SiO}_{4}^{-}$). Therefore, knowing that under the conditions used in our work most of the MAS Is actually monomeric, we analyzed the data as though all of it were. Strictly speaking, this is wrong, but, as will be discussed in S3.7, this convenient approximation does not hurt final results and is easy to compensate for in retrospect.

Reaction vessels. At $100^{\circ} \mathrm{C}$ the reaction vessel was a $1-\mathrm{L}$ three-necked flask, and $400 \mathrm{ml}$ of the solution (room temperature measure) was used. The acld solution was preheated in the flask, and the alkaline solution was preheated in a stainless steel vessel.

At temperatures below $100^{\circ} \mathrm{C}, 8-0 z$. polyethelene bottles (weighted with lead foll or solder on the outside when used in a water bath) were used for both preheating and the actual experiment.

All alkaline solutions were stored in plastic ware. However, measuring, mixing, etc. were routinely done using Pyrex glassware. Blank experiments demonstrated that our procedures were adequate to prevent changes in silica concentration due to reactions with glassware.

pH Measurement. We found that it is extremely difficult to prepare a solution to have exactly the $\mathrm{pH}$ value desired. Typlcally, we found resulting $\mathrm{pH}^{\prime} s$ after mixing to differ by $\pm 0.1 \mathrm{pH}$ unit from what we had intended them to be. When working with a new buffer system or new added salt, this discrepancy was sometimes as large as 0.3 units. Because of this, the solution $\mathrm{pH}$ was always measured at the end of the experiment and that value used in subsequent data analysis. A digital pH meter calibrated at the given temperature was employed. Preliminary tests revealed that the $\mathrm{pH}$ was stable to within about \pm 0.01 unit over the duration of a run. Some of the $100^{\circ} \mathrm{C}$ experiments were performed with a consistent error of about 0.15 unit in the meter calibration, which was corrected for during data analysis. We believe the most probable error in $\mathrm{pH}$ at this temperature to be about \pm 0.02 unit. 
An Orion Model 701A pH meter was used. In all cases, it was calibrated with a standard held at the temperature at which the actual measurements were to be made. It was found to be necessary to allow the electrode to thermally equilibriate for about 15 minutes in the warmed standard to obtain a stable reading. Until the electrode had been allowed to stabilize in this way, there was a slow drift in the reading. This drift was attributed to the relatively slow response of the $\mathrm{KCl}$ concentration in the saturated $\mathrm{KCl} /$ calomel reference electrode to the change in electrode temperature.

Experience showed that the electrode should not be left standing in the experimental solutions any longer than it took to obtain a reading. It was found that prolonged and/or frequent contact with the experimental solutions caused a deterioration in electrode performance over time which manifested itself as a progressively slower response. This was attributed to silica deposition on the glass membrane which progressively increased its electrical resistance. In general, an electrode of the sealed type is preferable for work above room temperature because ordinary, unsealed electrodes suffer from evaporation of the fluid in the KCl bridge.

\subsection{The Rate of Molecular Deposition as a Function of $\mathrm{pH}$}

A number of pairs and triplets of experiments were performed which differed only in pH. (No salts were added in this serles.) Experiments both with and withort added colloid were included in this series, and most were performed at $50^{\circ} \mathrm{C}$. A typical triplet of curves generated in this series is presented in Fig. 3.2. The expected accelerating effect of increasing $\mathrm{pH}$ is evident.

All data analysis was done in terms of pairs of curves run at the same temperature, initial silica concentration, and added collold (if any). Sets of three curves were treated as two pairs of curves at adjacent pH values. In most cases two curves that were compared with each other during data analysis had actually been run simultaneously.

The analysis of the colloid-added data in this series began with fitting a trial kinetic function to each curve and determining the empirical value of the apparent $\mathrm{pH}$ dependent rate constant from it. (That is to say, a "rate constant" whose value reflects the $\mathrm{pH}$ as well as the temperature. 


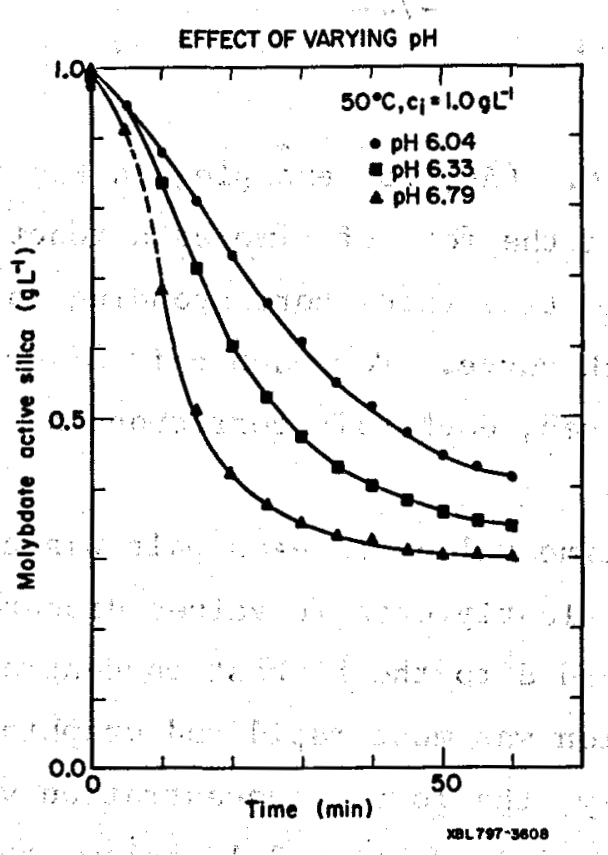

Fig. 3.2. Homogeneous nucleation experiments with barbital-maleate buffer. Throughout the Figures in this Chapter, all kinetic data like this were generated using BM buffer unless explicitly stated otherwise.

This fitting procedure is discussed in detail in 53.4 below.) The apparent kinetic order in respect to $\mathrm{OH}^{-}$was then calculated by using the approximate relation:

$$
\begin{aligned}
\mathrm{N}=\mathrm{d} \ln \mathrm{k} / \mathrm{d} \ln \mathrm{aOH}^{-}=\mathrm{d} \log \mathrm{k} / \mathrm{d} \mathrm{pH} \\
=\left(\log \mathrm{k}_{2}-\log \mathrm{k}_{1}\right) /\left(\mathrm{pH}_{2}-\mathrm{pH}_{1}\right)
\end{aligned}
$$

The value of $\mathrm{N}$ directly reflects the effect of $\mathrm{pH}$ change on the rate of molecular deposition unless the $\mathrm{pH}$ is so high that the dissociation of MSA is important.

The rate of polymerlzation by way of homogeneous nucleation is affected by the $\mathrm{pH}$ through the effect of $\mathrm{pH}$ on the rate of molecular deposition. It is also affected by the $\mathrm{pH}$ through the effect of $\mathrm{pH}$ on the surface tension (see s3.10) but, under the conditions of these experiments, this is only a secondary effect. Ignoring this secondary effect allows the effect of $\mathrm{pH}$ on the rate of molecular deposition to be extracted from the homogeneous nucleation data in a strictly analogous way.

The homogeneous nucleation data was initially gathered in the form of MAS concentrations determined at regular time intervals (typically 5 to 10 minutes). This data was first plotted on linear graph paper, and smooth 
curves were drawn through it. (As, for example, in Fig. 3.2.) It was then converted to tabular data in the form of time as a function of concentration. This was done by reading the time value corresponding to a given concentration from the graph for each curve. For each pair of curves, this gave a column of pairs of time values, each pair corresponding to a different concentration.

The ratio of the two time values in each pair was calculated. These figures were examined, and clearly erratic values discarded. These were usually those that corresponded to the highest concentration values, where the drop in MAS concentration was most rapid and graphing and interpolation errors greatest. Frequently, the lowest concentration values were also erratic. (As will be discussed in Section 3.12 below, this was probably due to a change in the particle growth mechanism under these conditions.)

Then the remaining values of the time ratio were averaged. An average time ratio calculated in this way is actually the reciprocal of the molecular deposition rates at the two given $\mathrm{pH}$ values. Finally, the value of $\mathrm{N}$ for the given pair of curves was calculated using the formula

$$
\mathrm{N}=-\log \left(\left(\mathrm{t}_{2} / \mathrm{t}_{1}\right)_{\text {aver }}\right) /\left(\mathrm{pH}_{2}-\mathrm{pH}_{1}\right)
$$

Values of $\mathrm{N}$ calculated from six pairs of colloid added curves and seven pairs of homogeneous nucleation curves at $50^{\circ} \mathrm{C}$ are presented in Fig. 3.3. Each bar corresponds to a pair of curves. The height of each bar represents the value of $\mathrm{N}$, and the positions of its two edges represent the $\mathrm{pH} s$ of the two curves.

The data in Fig. 3.3 is consistent with the generally accepted view that the rate of molecular deposition is proportional to the concentration of Ionized silanol groups on the silica surface. At low $\mathrm{pH}$, their concentration varies in proportion to the concentration of hydroxide ion in solution and $\mathrm{N}$ is about one. At $\mathrm{pH}$ values high enough for a significant fraction of the surface silanols to be dissociated, the increasingly negative surface potential makes the increase in the concentration of dissociated surface silanols lag behind the hydroxide ion concentration, and the value of $\mathrm{N}$ decreases.

Fitting the data. The experimental values of $N$ in Fig. 3.3 were fitted using an equation proposed by Allen et al. (1971): 


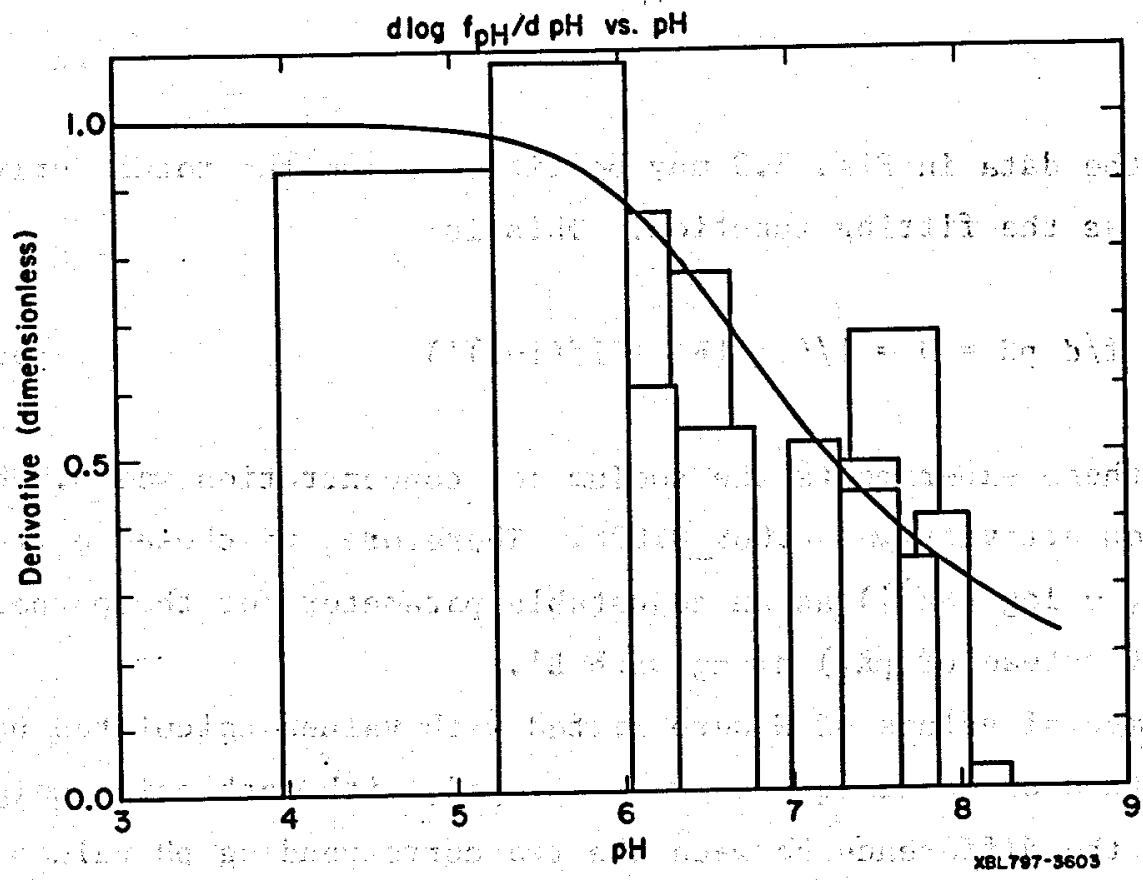

Fig. 3.3. The bars represent experimental values, and the colld curve represents a solution of Eqn. $(3.3 .3)$ calculated using the values of the two parameters in that equation which give the best fit.

$$
\log f=p H-\mathrm{pK}_{1}+\log \left[\mathrm{Na}^{+}\right]-\mathrm{B}^{\prime} \mathrm{f} / 2.302+\log (1-f)
$$

where

$$
\begin{aligned}
& f=\text { the fraction of the surface silanols that are dissoclated } \\
& \mathrm{pK}_{1}=\text { the "intrinsic", or low } \mathrm{pH} 1 \text { lmit, value of the } \mathrm{pK}_{\mathrm{a}} \text { of } \\
& {\left[\mathrm{Na}^{+}\right]=\text {the surface silanol groups }}
\end{aligned}
$$

and $B^{\prime}$ is proportional to the reciprocal of the inner layer capacitance. The detailed site binding model of Davis et al. (1978) also reduces to this single equation if one makes the the simplifying approximation that all dissociated silanols have sodium lons bound to them. (This is actually a good assumption at higher salt concentrations.)

The hypothesis that the rate of molecular deposition is proportional to f leads us to make the Identification

$$
\mathrm{N}=\mathrm{d} \log \mathrm{f} / \mathrm{d} \mathrm{pH}
$$


Therefore, the data in Fig. 3.3 may be fitted using the total derivative of $\log \mathrm{f}$ over $\mathrm{pH}$ as the fitting function. This is:

$$
d \log f / d p H=N=1 /\left(1+f\left[B^{\prime}+1 /(1-f)\right]\right)
$$

In most of these experiments the sodium ion concentration was $0.088+0.01$ and the sodium fon activity was $0.069 \pm 0.008$. Therefore, we chose to treat the quantity $\left(\mathrm{pK}_{1}-\log \left[\mathrm{Na}^{+}\right]\right)$as an adjustable parameter for the purposes of data fitting (instead of $\mathrm{pK}_{1}$ ) along with $B^{\prime}$.

The experimental values of $N$ were fitted with values calculated using (3.3.5). The method of least squares was employed, with each point weighed in proportion to the difference between the two corresponding $\mathrm{pH}$ values (1.e.; the width of the bar in Fig. 3.3). The values of $f$ on the R.H.S. were calculated by integrating (3.3.5) from low $\mathrm{pH}$, at which the initial value of $f$ was taken to be

$$
\log \mathrm{f}=\mathrm{pH}-\mathrm{pK}_{1}+\log \left[\mathrm{Na}^{+}\right]
$$

The best fit (weighed $\mathrm{r}$.m.s. error $=0.141$ ) was obtained with $B^{\prime}=5.2$ and $\mathrm{pK}_{1}-\log \left[\mathrm{Na}^{+}\right]=7.6$, which corresponds to $\mathrm{pK}_{1}=6.4$. This is in excellent agreement with values $B^{\prime}=4.3$ and $\mathrm{pK}_{1}=6.6$ found by $\mathrm{Allen}$ et al. (1971) when they fitted room temperature titration data for Ludox HS employing (3.3.3). This excellent agreement conclusively demonstrates that the rate of molecular deposition is proportional to the concentration of dissociated surface silanol groups. The solid curve in Fig. 3.3 represents the best fit.

Introducing a standard state. The form of (3.3.3) suggests that the ratio of the activities of the sodium and hydrogen ions determines the value of $f$ rather than their separate values. This suggests that $f$ may be more conveniently expressed as a function of some combination of $\mathrm{pH}$ and [ $\mathrm{Na}^{+}$]. We chose to work with $\mathrm{f}$ expressed as a function of "nominal $\mathrm{pH}$ ", defined as

$$
\mathrm{pH}_{\text {nom }}=\mathrm{pH}+\log \left(\left[\mathrm{Na}^{+}\right] / 0.069\right)
$$

When $\left[\mathrm{Na}^{+}\right]=0.069$ (as was approximately the case with most of our work), $\mathrm{pH}=\mathrm{pH}_{\text {nom }}$. 
Furthermore, most of our experiments were performed between pH 6 and 8 . This suggests that a convenient reference state for our purposes would be $\left[\mathrm{Na}^{+}\right]=0.069$ and $\mathrm{pH}=\mathrm{pH}_{\text {nom }}=7.0$. Therefore, we chose to work with the value of $f$ normalized relative to 1 ts value under these conditions. We define

$$
f^{\prime}\left(\mathrm{pH}_{\text {nom }}\right)=f\left(\mathrm{pH}_{\text {nom }}\right) / f(7.0)
$$

where

$$
f(7.0)=0.118913
$$

Because there are approximately seven silanol groups per square nanometer on the surface (see S3.10), th1s standard state corresponds to slightly less than one fonfzed silanol group per square nanometer.

Eqns.(3.3.3) and (3.3.5) are fundamental, but too unwieldy for rout ine practical application. Therefore, we refitted the values of $f$ calculated from (3.3.5) with arbitrary closed form analytic expressions. These expressions are presented in Appendix A3.1. They appear in FORTRAN coded form in SUBROUTINE PHF of SILNUC as well (see Chapter 6). Figure 3.4 presents the value of $f^{\prime}$ calculated using these expressions, as does Table A3.1 in Appendix A3.1.

The separate effects of $\mathrm{pH}$ and $\mathrm{pH}$ nom. Much later, when the effect of added sodium chloride on the homogeneous nucleation process was studied in deta11 (see 53.12), It was found that Eqn. (3.3.3) alone was not able.to reconclle the high and low salinity data. It turned out that the assumption implicit in (3.3.3) that all of the lonlzed surface silanols have sodium lons bound to them is incorrect. Apparently, in a low salinity medium (like our "buffer only" experimental solutions) a substantial fraction do not. This becomes obvious when an attempt is made to reconcile kinetic data obtained at different salt concentrations.

The concentration of these "unpaired" dissociated silanols is more-orless Independent of the sodium ion activity. Therefore, the expression for the surface density of dissoclated silanols should contain a term which depends only on $\mathrm{pH}$ (rather than $\mathrm{pH}_{\text {nom }}$ ). Unfortunately, this was discovered much too far along in the data reduction process for us to be able to go 


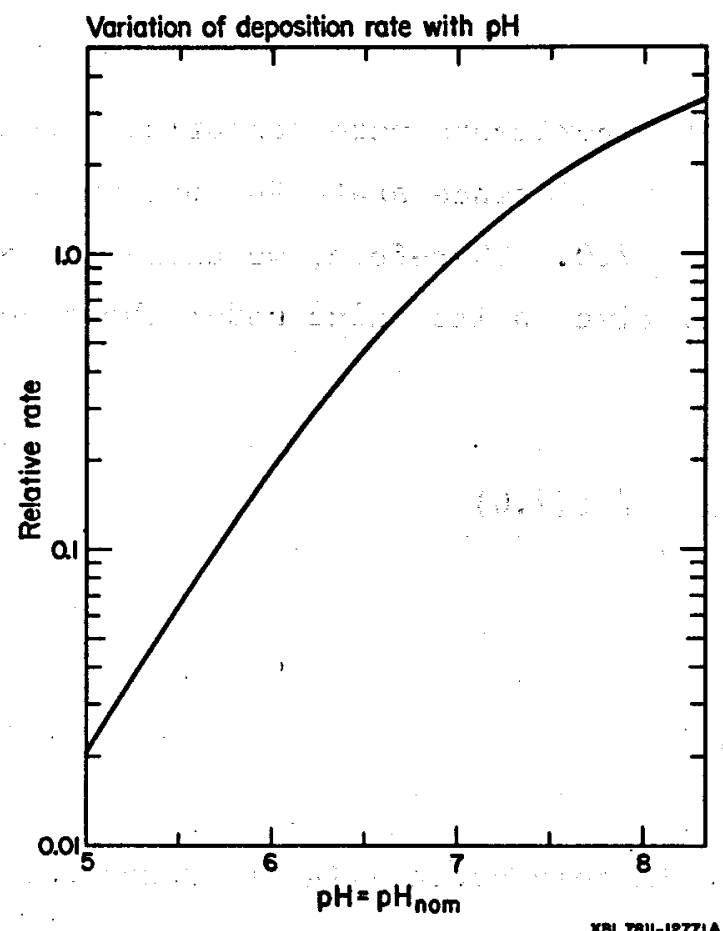

F1g. 3.4. The function plotted here is $\mathrm{f}^{\prime}(\mathrm{pH})$, which $\mathrm{F}\left(\mathrm{pH}, \mathrm{pH}_{\mathrm{nom}}\right)$ is equal to when $\mathrm{pH}:=\mathrm{pH}_{\text {nom }}$.

back and start over using a more adequate replacement for (3.3.3). Instead, we chose to proceed with the simplifying (and simplistic) assumption that the function that expresses the dependence of the reaction rate on $\mathrm{pH}$ and salinity could be written in the form:

$$
F\left(p H, p_{n o m}\right)=h f^{\prime}(p H)+(1-h) f^{\prime}\left(p^{n o m}\right)
$$

where $h$ is yet another adjustable parameter to be fitted to the experimental data. The high salinity data was best fitted using $h=0.45$ (see S3.12). When $\left[\mathrm{Na}^{+}\right]=0.069$, which was approximately the case in most of our work, $\mathrm{pH}=\mathrm{pH}_{\mathrm{nom}}$, and $\mathrm{F}=\mathrm{f}^{\prime}$. This is why ignoring the "unpaired" dissociated silanols had little effect on the subsequent data analysis until we got to the high salinity data.

of course; theoretical models of the ion exchange properties of the amorphous silica surface much more advanced than that implied in (3.3.9) are available; in particular, the "site- binding" model of Davis et al. (1978). However, (3.3.9) does seem to fit our data almost to within experimental error, and, the substantially greater complexity of a physically more realistic treat- 
ment did not seem warranted. Also, note that the (3.3.9) becomes more realIstic as the salinity is increased and the $\mathrm{pH}$ dependent term becomes less important. In practice, all this means that $(3.3 .9)$ can be used with confidence whenever the sodium ion activity is equal to or greater than 0.069 .

Sources of error. Several other minor inconsistencles in the analysis of the $\mathrm{pH}$ effect data were allowed to remain by virtue of having been discovered much too late to do anything about them. First, the two lowest pH experiments included in Figure 3.3 (those which correspond to the vertical Iines at $\mathrm{pH} 3.96$ and 5.24) were performed using citrate buffer, which gives a somewhat lower sodium ion activity than does the barbital-maleate buffer system used at higher $\mathrm{pH}^{\prime} \mathrm{s}$. Therefore, the approximate equality of $\mathrm{pH}$ and $\mathrm{pH}_{\mathrm{nom}}$ does not hold for these experiments, and this was not compensated for in data analysis. This is the probable cause of the spuriously high bar between $\mathrm{pH}$ 5.24 and 6.04 . Fortunately, the mathematical form of the function being fitted made the results relatively insensitive to the empirical values of $\mathrm{N}$ in this low pH range.

Second, although the intent here was to determine the effect of $\mathrm{pH}$ on the rate of molecular deposition, data from homogeneous nucleation experiments were also used in compiling Figure 3.3. The rate of this process is also subject to another accelerating effect of increasing $\mathrm{pH}$ that is mediated by the decrease in surface tension associated with increasing surface charge density (see S3.10). Fortunately, all but one of the seven bars in Fig. 3.3 that are above pH 7 correspond to molecular deposition (1.e., "colloid added") experiments and were not affected by this problem. The one homogeneous nucleation experiment in this pH range shows a significant deviation in the expected direction (the tallest bar). The rest of the homogeneous nucleation data was generated at lower $\mathrm{pH}$ values at which the surface tension lowering effect is smaller and varies less with $\mathrm{pH}$.

Finally, the increase of solubility with increasing pH has not been compensated for. This may have been the cause of the very short bar above $\mathrm{pH} 8$. However, this one spurlously low value seems to have had little or no effect on the overall quality of the fit.

The effect of cations other than sodium is discussed in S3.13.

A few experiments like this were done at temperatures other than $50^{\circ} \mathrm{C}$. Unfortunately, these results were not extensive enough to establish anything 
more than that the $\mathrm{pH}$ dependence at the other temperatures is comparable to that at $50^{\circ} \mathrm{C}$. Therefore, the formulas and tables presented in Appendix A3.1 are recommended for use at all temperatures for the time being.

Polymerization rates at high $\mathrm{pH}$. Figure 3.5 shows the influence of $\mathrm{pH}$ on the rate of molecular deposition in the $\mathrm{pH}$ range above 8 . The deposition rate is roughly independent of $\mathrm{pH}$ in the $\mathrm{pH}$ range 8 to 9 , and decreases with further increases in the $\mathrm{pH}$ value above 9. The surface density of ionized silanol groups increases with increasing pH throughout; however, above about pH 8 the solubility of amorphous silica also begins to increase rapidly with increasing $\mathrm{pH}$ because of the lonic dissociation of MSA, and this has a compensating decelerating effect. Between about $\mathrm{pH} 8$ and 9 , the two effects cancel almost perfectly, and the rate of molecular deposition is approximately independent of $\mathrm{pH}$. At even higher $\mathrm{pH}$ values the decelerating effect is dominant and the rate decreases.

Fig. 3.6 shows the effect of Increasing $\mathrm{pH}$ above about 8 on the homogeneous nucleation process. There is a slight increase in the overall rate up

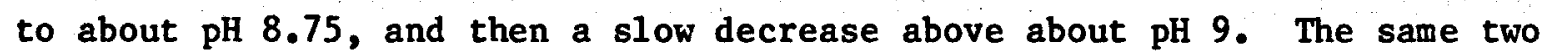
factors are operative as with the data in Fig. 3.5 and approximately cancel each other out here as well. The $\mathrm{pH}$ also affects the free energy of formation of the critical nucleus. The increasing solubility of silica decreases the saturation ratio at a given concentration of MAS, and this tends to slow down nucleation. The increasing extent of surface ionization reduces the surface tension, and this tends to accelerate nucleation. These two effects approximately cancel each other out as well.

The reader is cautioned that these are really only qualitative examples that refer to one particular temperature and range of salinity and MAS concentration. This general pattern certainly holds for other temperatures, etc., but the rate plateau need not always be in the same $\mathrm{pH}$ range, etc. However, all of these effects may be numerically modeled using the computer code SILNUC which is presented and discussed in Chapter 6 of this report. Unfortunately, the data in Figures 3.5 and 3.6 were not integrated into the overall data reduction process for lack of time. Therefore, even though SILNUC can be used to make predictions for the high pH range, these predictions will actually be extrapolations and should be recognized as such. 


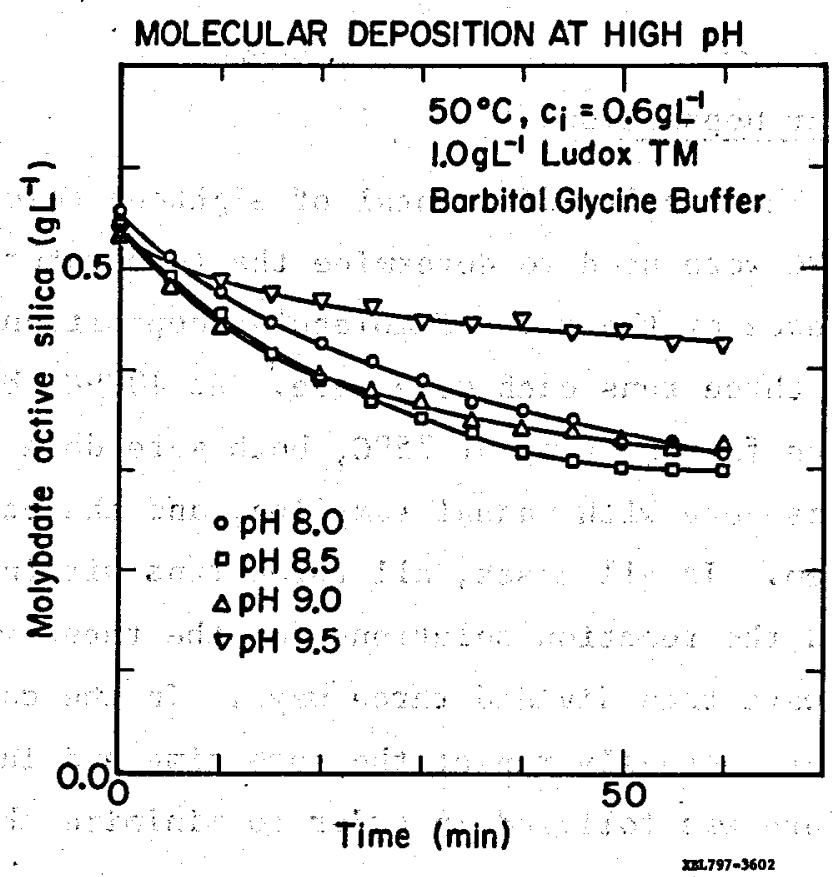

Fig. 3.5. Data from a "colloid added experiment" . Throughout, the amount and type of added colloid are specifled directly on the Figures. If colloid is not specifled, that experiment was of the homogeneous nucleation type.

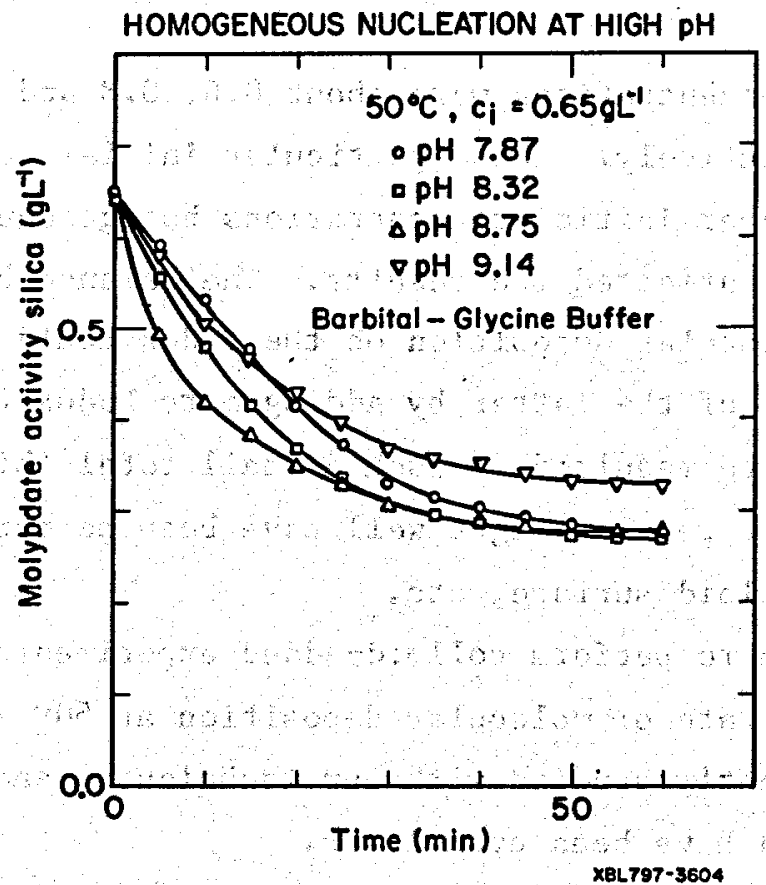

Fig. 3.6. 


\section{S3.4 The Rate of Molecular Deposition}

Experimental data. The results of a total of eighteen selected experiments at 50,75 , and $100^{\circ} \mathrm{C}$ were used to determine the temperature and MAS concentration dependence of the rate of molecular deposition. At each temperature, two sets of three runs each were made. At $100{ }^{\circ} \mathrm{C}$, both sets were run on the continuous flow system; at $75^{\circ} \mathrm{C}$, both were done with manual sampling; at $50^{\circ} \mathrm{C}$, one was done with manual sampling, and the other with the continuous flow system. In all cases, all three runs within a set were done on the same day, and the reaction solutions for the three were prepared as single batches which were then divided three ways. In the case of the manual runs, the three were actually. run at the same time and in the same water bath. This procedure was followed in order to minimize the random variations from run to run within each set.

The runs within each set differed only in the type and amount of colloidal silica introduced into each solution. The three formulations were: $1 \mathrm{~g} \mathrm{~L}^{-1}$ Ludox TM, $0.6 \mathrm{~g} \mathrm{~L}^{-1}$ Ludox HS, and $0.4 \mathrm{~g} \mathrm{~L}^{-1}$ Ludox SM. In each case, this corresponded to a total surface area of between 143 and $157 \mathrm{~m}^{2} \mathrm{~L}^{-1}$.

The initial MAS concentrations were about $0.6,0.8$ and $0.85 \mathrm{~g} \mathrm{~L}^{-1}$ at 50,75 , and $100^{\circ} \mathrm{C}$, respectively. These particular initial concentrations were used because at higher initial concentrations homogeneous nucleation would have significantly affected the results. The balance between homogeneous nucleation and molecular deposition on the Ludox could have been shifted further in favor of the latter by adding more Ludox (considerably more), but this would have resulted in such a small total thickness of deposited silica that the results might well have been perturbed by the initial state of the colloid surface, etc.

No attempt was made to perform collold-added experiments at temperatures below $50^{\circ} \mathrm{C}$, because the rate of molecular deposition at $50^{\circ}$ is about as small as can be conveniently studied with our techniques, and the rate at lower temperatures would have been even lower.

Figure 3.7 presents the data from a continuous flow system experiment. Figure 3.8 presents the results of a set of three manually sampled runs. 


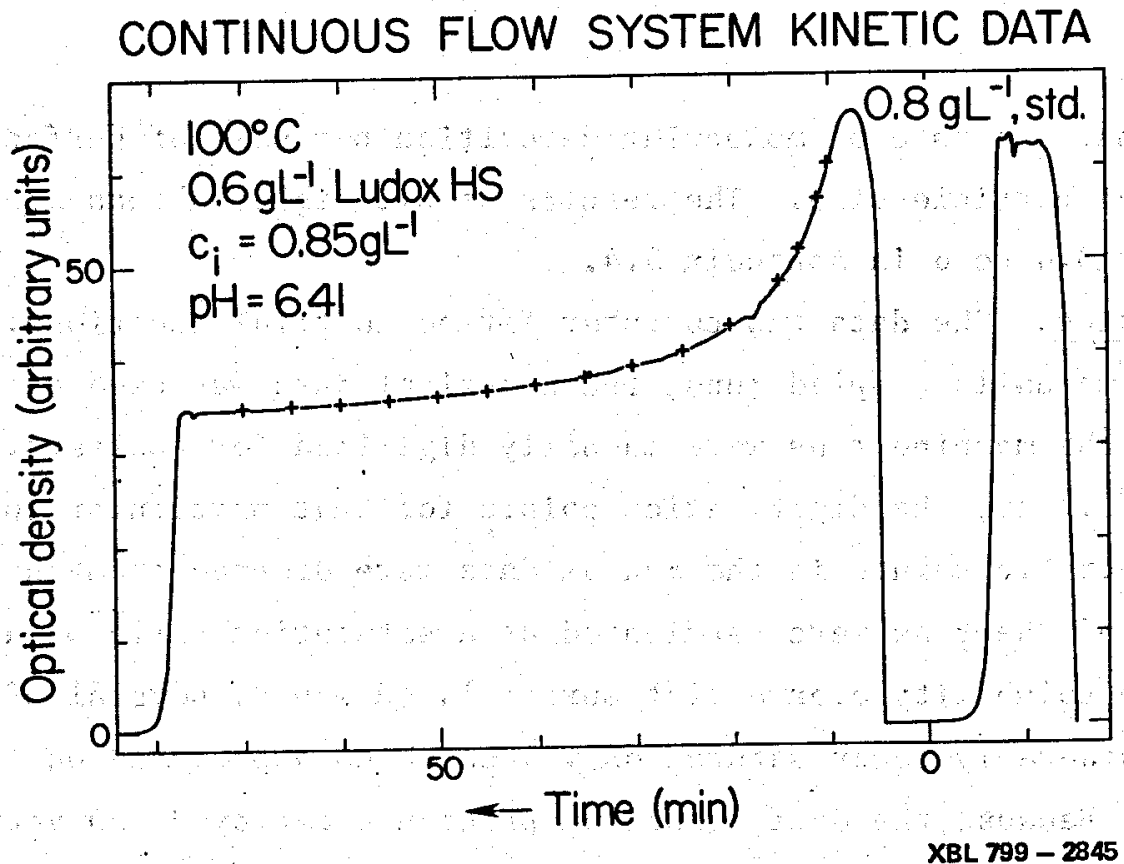

Fig. 3.7. The crosses superimposed on the curve are manually placed digitization points whose coordinates were then read to convert the curve to digital form.

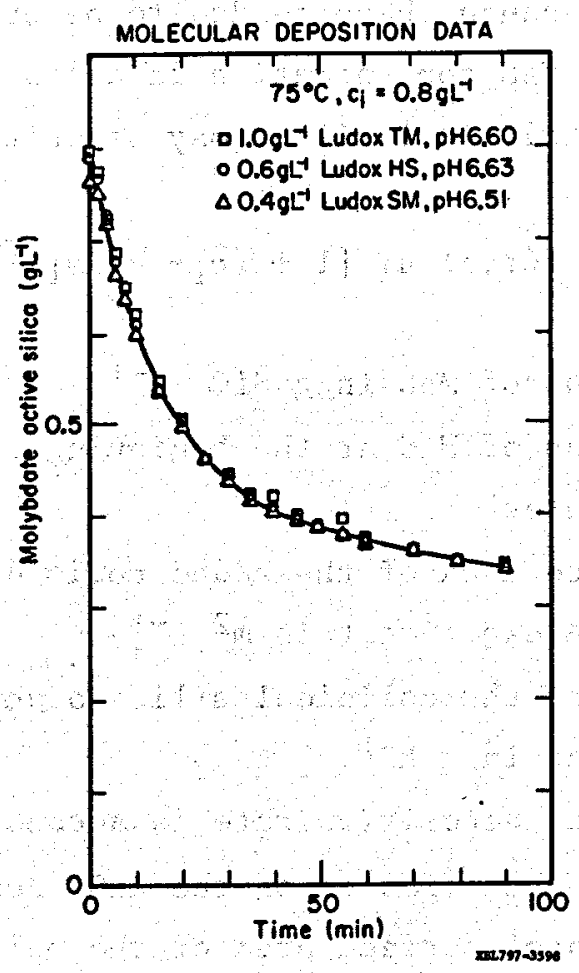

$\mathrm{Fig} \cdot 3.8$ 
It is evident that the rate of molecular deposition per unit of surface area is independent of particle size. The results of all elghteen runs are presented in Table A3.4 to 6 in Appendix 3.4.

Data reduction. The data was computer fitted to trial functions. In the case of the manually sampled runs, the numerical data was used directly. The curves from the machine runs were manually digitized for analysis. The crosses in Fig. 3.7 are the digitization points for that particular run. Some obviously erratic points in the manual data were dropped from the fitting process. Most of the runs were terminated at a saturation ratio of about 1.28 (relative to the solubility over a flat surface). However, several of the $100^{\circ} \mathrm{C}$ runs ran to considerably lower saturation values which approached equilibrium with the Ludox. Because the data reduction procedure employed was very sensitive to the low concentration portion of the curve, including these "tail" points in the analysis might have caused spurious fits that were no good at all in the more interesting higher concentration range. Therefore, points below $S=1.28$ were deleted from the fitting process. The very first point in each run was also dropped because these tended to be erratic.

The rate of decline of MAS concentration in a low salinity medium contalning monodisperse added colloidal sillca may be written as

$\mathrm{dc} / \mathrm{dt}=$

$$
\text { - } k(Y, T) F\left(p H, p H_{n o m}\right) f(Y, c) a_{1}\left[1+\left(c_{1}-c\right) / m_{1}\right]^{(2 / 3)}
$$

where

c = the concentration of MAS in $\mathrm{g} \mathrm{SiO}_{2} \mathrm{~L}^{-1}$

$c_{1}=$ the concentration of MAS at the beginning of the experiment

$t=$ the time in minutes

$a_{1}=$ the total surface area of the added colloidal silica at the beginning of the experiment in $\mathrm{m}^{2} \mathrm{~L}^{-1}$

$m_{1}=$ the total mass of the colloidal silica added at the beginning of the experiment in $\mathrm{g} \mathrm{L}^{-1}$

The $\mathrm{pH}$ dependence of the deposition rate is accounted for by the explicit factor $F\left(\mathrm{pH}, \mathrm{pH}_{\mathrm{nom}}\right)$. The last factor on the RHS accounts for the effect of particle growth on the total surface area of the colloid that is present. $f(Y, c)$ is the function which actually describes the dependence of the rate of molecular deposition on $c$. The functional form of $f$ is determined 
by one or more parameters symbolized by $Y$ which are to be varied in searching for the best fit.

$k(Y, T)$ is the $\mathrm{pH}$ and concentration independent "rate constant". The numerical value of $k$ is, of course, influenced by the form of $f(Y, c)$ and the value (s) of $Y$.

Rearrang Ing and Integrating (3.4.1), we obtain

$t_{1}-t_{1}$

$$
=-\left[k(Y, T) F\left(p H, p_{n o m}\right)\right]^{-1} \int_{c_{1}}^{c_{1}}\left[a_{1} f(Y, c)\right]^{-1} d c
$$

where the subscript 1 refers to a specific data point; $1 . e_{.}, c_{1}$ was the concentration of MAS measured at $t_{1}$ and so on.

$$
a_{1}=a_{1}\left[1+\left(c_{1}-c_{1}\right) / m_{1}\right]^{(2 / 3)}
$$

The Integral on the RHS of (3.4.2) was numerically evaluated for each $c_{1}$ using the trial function $f(Y, c)$. (Simpson's rule was used with each interval $c_{1+1}-c_{1}$ divided into twenty parts.) A linear regression was then performed; the empirical values of the LHS were fitted as a Iinear function of the numerically calculated values of the RHS. $k(Y, T) F\left(p H, p H_{n o m}\right)$ is equal to the reciprocal of the slope. This is the "pH dependent rate constant" alluded to in S3.3. In the experiments described there, conditions were such that the value of $k(Y, T)$ was the same in all cases. This allowed the variation of $\mathrm{F}\left(\mathrm{pH}, \mathrm{pH}_{\mathrm{nom}}\right)$ with $\mathrm{pH}$ to be determined. In the experiments described here, $\mathrm{F}\left(\mathrm{pH}, \mathrm{pH}_{\mathrm{nom}}\right)$ was already known, and the results were used to determine the value of $k(Y, T)$. Mathematically, this procedure is completely analogous to that used to analyse kinetic data for ordinary liquid and gas phase reactions.

Trial rate functions. Applying the Law of Microscopic Reversibility in the form usually applied to the analogous problem in the theory of vapor condensation (Abraham, 1974, pp.80-83), it is easy to show that the rate function should have the form

$$
f(Y, c)=f_{f}(Y, c)\left[1-S^{-1} \exp \left(2 \gamma /\left(\rho_{n} k_{B} T r\right)\right)\right]
$$


where

$f_{f}(Y, c)=$ the rate of the forward reaction alone, (1.e., the rate of deposition not corrected for simultaneous redissolution) divided by $k(Y, T)$

$\mathrm{S} \quad=$ the saturation ratio; $1 . e .$, the concentration of dissolved silica divided by the equilibrium solubility under the given conditions of temperature, salinity, and $\mathrm{pH}$

$\gamma .=$ the surface tension in ergs $\mathrm{cm}^{-2}$

$\rho_{n} \quad=$ the number density of AS $=2.21 E 22 \mathrm{~S} 10_{2}$ units $\mathrm{cm}^{-3}$

$k_{B}=$ the Boltzman constant $=1.38054 \mathrm{E}-16$ ergs $K^{-1}$

$\mathrm{T}=$ the absolute temperature in Kelvins

$r \quad=$ the particle radius in centimeters

The exponential factor serves to correct the value of $S$ to account for the increased solubility of small particles.

Eqn. (3.4.3) requires that the molecular deposition rate vary with particle size. However, the hypothesis of a particle size dependent deposition rate disagrees with our data, which shows that the rate is independent of particle size (see Fig - 3.8). No amount of forced fitting or compensation elsewhere could make (3.4.3) consistent with both the molecular deposition and homogeneous nucleation data. (This is discussed further in s3.10).

Therefore, we chose to instead use the following related form in which $r$ does not appear:

$$
f(Y, C)=f_{f}(Y, c)\left[1-S^{-1}\right]
$$

For reasons that are discussed in S3.5, we chose to treat $f_{f}$ as a function of

$S_{a}=(1-\alpha) c / c_{o}$

where

$\alpha=$ the fraction of the dissolved silica that is in ionic form

$c_{0}=$ the equilibrium solubility of amorphous silica in pure water at the given temperature in $\mathrm{g}\left(\mathrm{kg} \mathrm{H}_{2} \mathrm{O}\right)^{-1}$. 
Thus, $S_{a}$ is the saturation ratio corrected for the effect of $\mathrm{pH}$, but not for the effect of salinity. In low salinity media like our experimental solutions, $S_{a}$ is approximately equal to $S$.

We found that the molecular deposition data alone could be fitted reasonably well using the simple form

$$
f_{f}(S)=S_{a}^{n}
$$

The $50^{\circ} \mathrm{C}$ data was best fitted with $\mathrm{n}=4$, the $75^{\circ}$ data with $\mathrm{n}=5$, and the $100^{\circ}$ data with $n=6$, but the quality of the fit at 50 and $100^{\circ} \mathrm{C}$ was not very much degraded by using $n=5$ at these temperatures as well. Therefore, we chose to use $n=5$ throughout in order to simplify matters.

As will be discussed in 53.10 , it was not possible to fit the homogeneous nucleation data using this "fifth order" rate law. This contradiction is only apparent rather than real, because in most of the homogeneous nucleation experiments the initial concentration of MAS was considerably higher than in the collold-added experiments at the given temperature. Therefore, the two bodies of data reflect the behavior of $f_{f}$ in different ranges of the value of $\mathrm{S}_{a}$, and there is no reason to doubt that that behavior can vary with silica concentration.

Final results. The two bodles of data were best reconciled by assuming a fifth order rate law up to a certain "threshold" value of $S_{a}$ which we w111 call $s_{t}$, and a "first order" rate law above $s_{t}$, with continuity of the value and the slope at $s_{t}$ :

$$
\begin{aligned}
& f_{f}\left(s_{a}\right)=s_{a}{ }^{5} \quad \text { if } \quad s_{a}<s_{t} \\
& f_{f}\left(s_{a}\right)= \\
& s_{t}{ }^{5}+5 s_{t}^{4}\left(s_{a}-s_{t}\right) \quad \text { if } s_{a}>s_{t}
\end{aligned}
$$

where

$S_{t}=\operatorname{ant} 1 \log _{10}(0.0977+75.84 / \mathrm{T})$

Eqn. $(3.4 .7 \mathrm{c})$ gives the values $2.15,1.99$, and 1.85 at 50,75 and $100^{\circ} \mathrm{C}$, respectively. These correspond to dissolved silica concentrations of $0.389,0.524$, and $0.673 \mathrm{~g} \mathrm{~L}^{-1}$, respectively. In each case, th1s is considerably higher than the Inftial concentration used in the colloid-added experiments at the given temperature. However, the value of $s_{t}$ assumed has little effect on the quality of the fit to the results of a colloid added experiment or on the value obtained for the rate constant because the fitting procedure represented by $(3.4 .2)$ is relatively insensitive to the data at 
higher dissolved silica concentrations (1.e., $s_{a}>s_{t}$ ). The values of $S_{t}$ represented by $(3.4 .7 \mathrm{c})$ were f1tted using homogeneous nucleation data; this is described in detall in s3.10.

The rate of dissolution was not studied experimentally by us, and the spotty dissolution rate data avallable from other sources is not really adequate for our purposes. However, numerical modelling of the chemistry of amorphous silica requires that the rate of dissolution be defined in a numerical sense even if the actual physical value is unknown. We chose to use an arbitrary but convenient "first order" rate law for the dissolution process in the code SILNUC:

$$
\begin{aligned}
f(Y, C)=S- & \exp \left[2 \gamma /\left(\rho_{n} k_{B} T r\right)\right] \\
& \text { if and only if } \exp \left[2 \gamma /\left(\rho_{n} k_{B} T r\right)\right]>s
\end{aligned}
$$

Hote that there is a discontinuous jump in the value of $f(Y, c)$ as calculated using $(3.4 .7 a$ or $b)$ and $(3.4 .7 d)$ at $\exp \left[2 \gamma /\left(\rho_{n} k_{B} T r\right)\right]=s$. Th1s discontinuity may or may not be physically significant. (Note that the arbitrary expression (3.4.7d) is not the one at fault here.)

A value of the rate constant $k(Y, T)$ (which will henceforth be called $\mathrm{k}_{\mathrm{OY}}(\mathrm{T})$ ) was calculated from the results of each of the eighteen colloid added experiments. These eighteen values were fitted as a function of $1 / \mathrm{I}$ by linear regression with the result

$\log \mathrm{k}_{\mathrm{OH}}(\mathrm{T})=3.1171-4296.6 / \mathrm{T}$

where $k_{\mathrm{OH}}(\mathrm{T})$ has the units of $\mathrm{g} \mathrm{cm}^{-2} \mathrm{~min}^{-1}$.

The r.m.s. deviation of the eighteen empirical values of $\log k_{0 H}(T)$ from this line is only 0.038 . This residual scatter is shown in Fig. 3.9. Note the narrow spread among the points overall, and the two essentially coincident triplets of points at 50 and $100^{\circ} \mathrm{C}$ (continuous flow system data in both cases). Additional intermediate results from the fitting process are presented in the Tables in Appendix 3.4.

In summary, the molecular deposition rate itself is to be calculated as

$$
\begin{aligned}
& \mathrm{R}_{\mathrm{md}}\left(\mathrm{g} \mathrm{S10} \mathrm{Cm}^{-2} \mathrm{~min}^{-1}\right) \\
& =\mathrm{F}\left(\mathrm{pH}, \mathrm{PH}_{\mathrm{nOm}}\right) \mathrm{k}_{\mathrm{OH}}(\mathrm{T}) \mathrm{f}_{\mathrm{f}}\left(\mathrm{S}_{\mathrm{a}}\right)\left(1-\mathrm{S}^{-1}\right)
\end{aligned}
$$

The rate of molecular deposition over a broad range of values of temperature and $c(1-\alpha)$ in a low salinity medium with $\mathrm{pH}=\mathrm{pH}_{\text {nom }}=7.0$ is presented in Figure 3.10. The rate values in Fig. 3.10 were calculated with $\mathrm{S}$ 


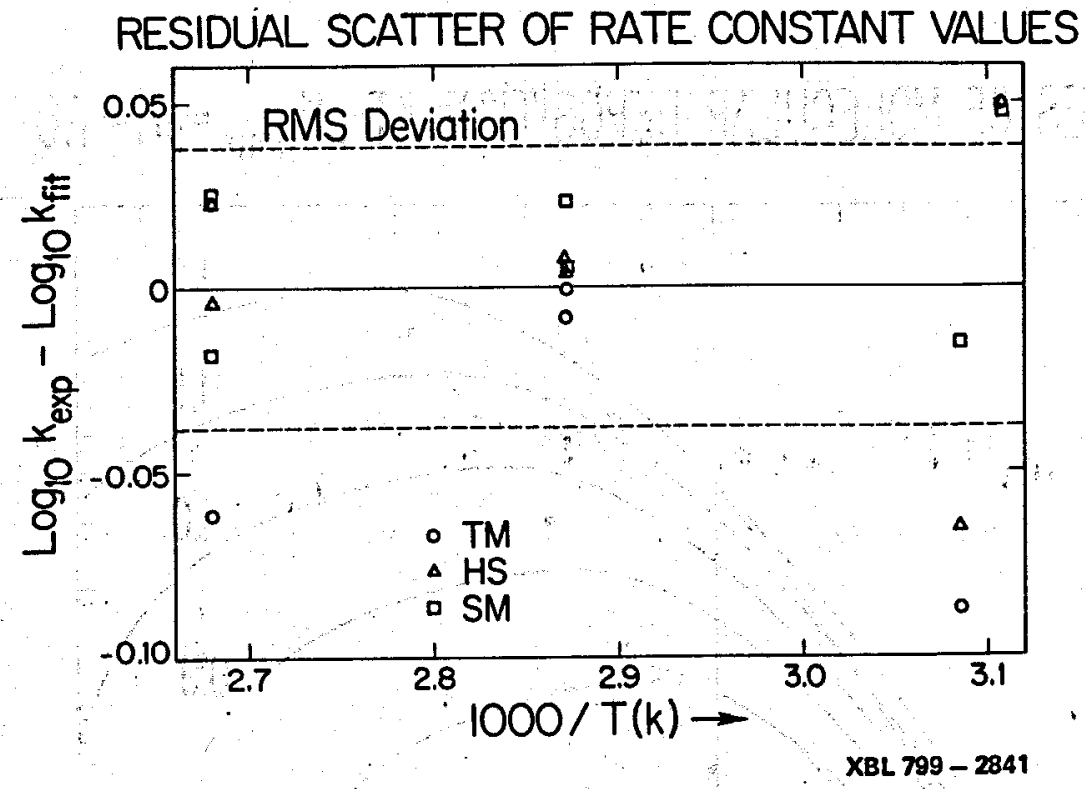

Fig. 3.9. Each point represents a molecular deposition experiment.

set equal to $S_{a}$, which is approximately true only in low salinity media. To correct the values read from Fig. 3.10 for this, multiply them by $(1-1 / s) /\left(1-1 / s_{a}\right)$. Usually, this correction factor is so close to one that it may be dispensed with, but it can be important when $S$ is close to one.

- The area outlined in the Figure with light solid lines is approximately that covered by our experimental data; the rest of the Figure is based on extrapolation. However, we believe even these extrapolated values to be considerably better than any other values, determined experimentally or otherwise, that are presently available.

The dotted Iine in the figure represents the boundary between the domains of fifth and first kinetic order. These lie to the right and to the left of it, respectively. It is striking how closely this line follows the locus of the maximum deposition rate value as a function of temperature at any given value of $c(1-a)$. This may or may not have a deeper physical significance. 


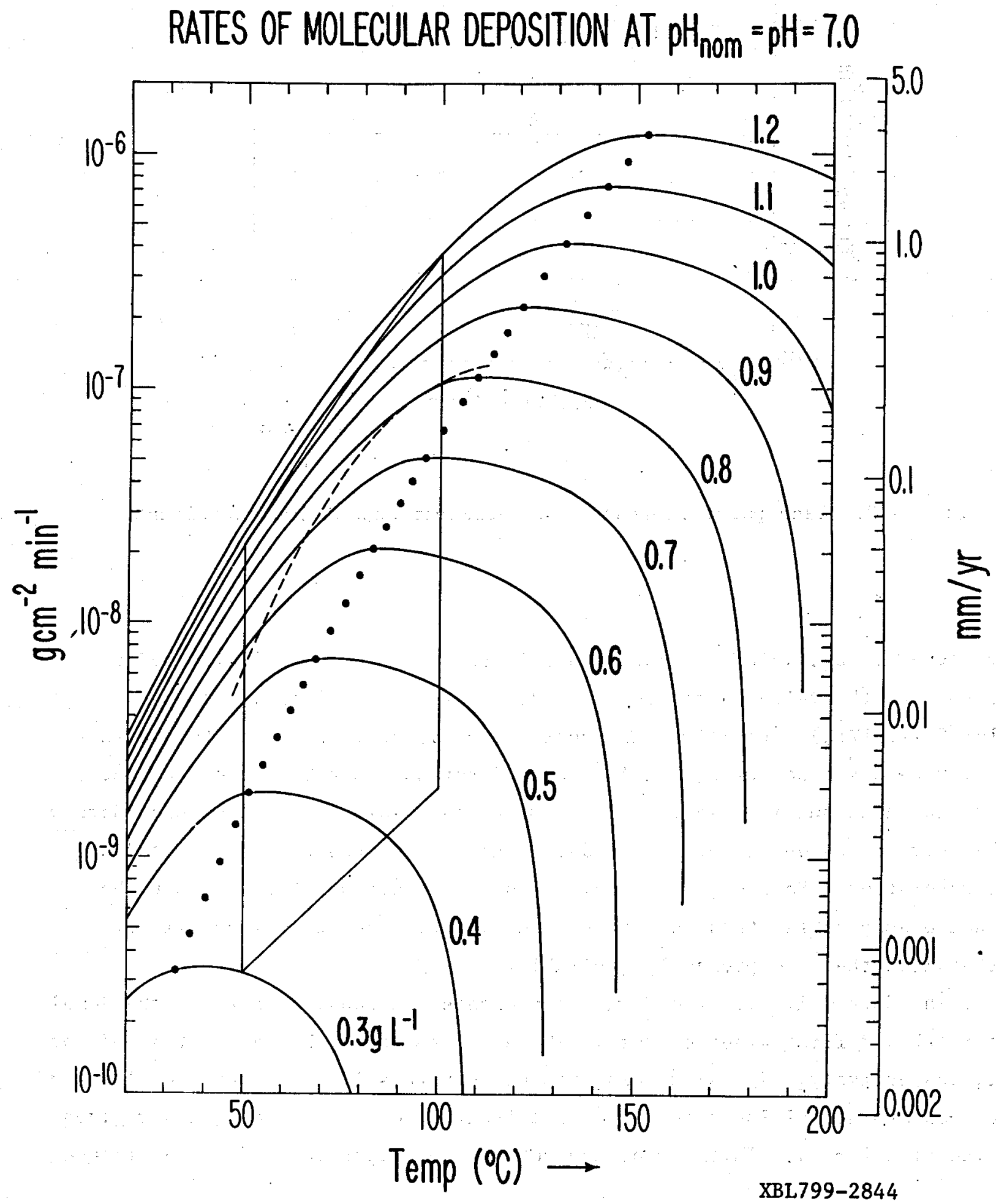

Fig. 3.10. Each solid curve is labeled with the corresponding concentration of dissolved silica in undissociated form; $1 . e ., c(1-\alpha)$. Al so, see the discussion of concentration units in S3.2. The light quadrilateral, dashed line, and dotted line are discussed, in the text in 53.4 . 


\section{S3.5 "Adsorbed S1lica" and the Structure of the Silica Surface}

We take the term "adsorbed silica" to mean what Holt and King :(1955) referred to as "adsorbed silicic acid" - that small amount of "loosely bound" silica on the surface of AS that leaches off the surface very rapidly in an alkaline medium. We do not mean to imply adsorption in the usual sense.

In practice, we determined adsorbed silica as follows: a quantity of crushed vitreous silica of known surface area was allowed to "equilibrate" with a solution containing a known concentration of MAS. Then the vitreous s1lica was leached with an alkaline solution. The rate at which the silica Initially went into solution was relatively high, but quickly dropped to a nearly constant limiting value. The amount of "adsorbed silica" was taken to be equal to the amount of silica that leached of early in the leaching process over and above the steady state rate of dissolution that was ultimately attained. The experimental technique is discussed in detail in A3.2.

Although our experiments were different from those of Holt and $\mathrm{KIng}$, we believe that they measured approximately the same physical quantity.

Figure 3.11 presents the values of the surface density of "adsorbed silica" at $50^{\circ} \mathrm{C}$ as a function of MAS concentration in the solution from which it was adsorbed. The experiments could not be carried out at concentrations above about $0.4 \mathrm{~g} \mathrm{~L}^{-1}$ MAS because at higher concentrations, homogeneous nucleation intervened.

The solid line In Figure 3.11 was drawn to intersect the concentration axis at $0.183 \mathrm{~g} \mathrm{~L}^{-1}$, which is the equilibrium solubility at $50^{\circ} \mathrm{C}$. The data are clearly consistent with the hypothesis that this is the true intersection point. This shows that here we are not dealing with an adsorption process in the usual sense. If it were, the equilibrium solubility value would have no special significance, and the plot of "adsorbed silica" versus - MAS concentration would intersect the concentration axis at zero concentration. However, the relationship observed is completely consistent with the hypothesis that the "adsorbed silica" is a reaction intermediate between dissolved silica and solid AS, and is present only when the molecular deposition process is occuring.

The apparent absence of "adsorbed silica" under conditions of dissolution (1.e., $s<1$ ) demonstrates that the dissolution process ts not simply the 


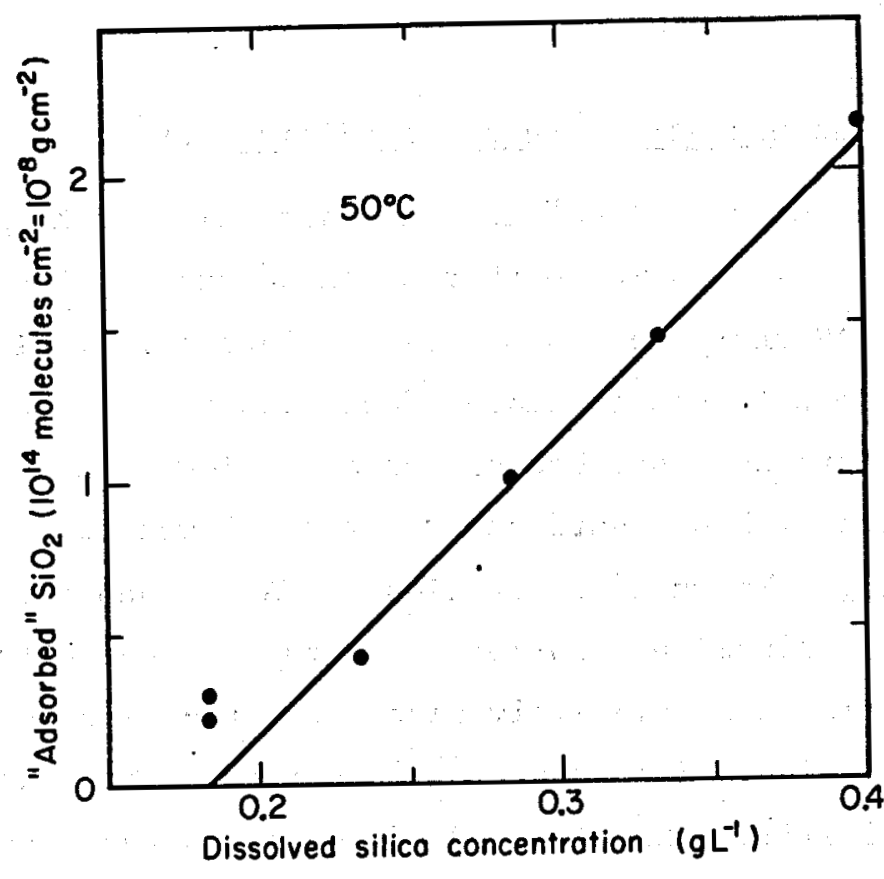

XBL7 $797-3600$

Fig. 3.11. Concentration of "adsorbed silica" on the surface of AS as a function of the concentration of MAS in solution.

molecular deposition process "running backwards." In other words, the Law of Microscopic Reversibility in its simple form does not apply to the molecular deposition process. This law must, of course, apply to the process at the level of the formation and fission of single chemical bonds; however, the overall process is much more complicated than fust this, and the Law does not apply to it as a whole. This is consistent with the observed independence of molecular deposition rate from particle size that was discussed in $\$ 3.4$.

The "adsorbed silica" must consist of primary and secondary silica groups; otherwise, it would not be as easy to leach off as it is. Therefore, an increase in the surface density of "adsorbed silica" must correspond to an increase in the number of primary and secondary groups on the surface and a decrease in the number of tertiary groups and in the extent of "siloxanic" (i.e., silanol free) areas. This change in the surface structure must involve an increase in the surface density of stlanol groups. Because the amount of "adsorbed silica" increases with increasing MAS concentration in solution, so must the surface density of silanol groups.

This change in the surface density of silanol groups is also evident from a comparison of estimates of the surface density of exchangeable protons 
$\left(n_{0}\right)$ obtained by different methods under different conditions: Titrating colloidal, sllica sols, Heston, Iler, and Sears (1960) estimated $n_{0}$ to be about $3.5 \mathrm{E} 14 \mathrm{~cm}^{-2}$, and the titration data of Allen and Matijevic (1970) indicates a value of about $3.9 \mathrm{E} 14 \mathrm{~cm}^{-2}$. Our own estimate, derived from homogeneous nucleation data, is about 7E14 (see S3.10). The values derived from titration data probably reflect the surface structure of AS that $1 \mathrm{~s}$ slowly dissolving. (In such titration work, the runs usually begin at low pH and end at $\mathrm{pH} 11$ or 12.) In this state, there 1 s no "adsorbed sillca" present, and the surface stlanol density has a minimum value. Our value applies to the silica surface under conditions of large supersaturation and rapid molecular deposition. Under these conditions, the amount of "adsorbed silica" and the number of surface silanols are probably at or near their maximum values.

These extremes of surface silanol density are easy to visualize. The varlous (hydrated) crystal surfaces of the crystalline sillca allotropes are convenient Idealized "model systems." (See, for example, the pictorial representations and extensive discussions presented by Iler, 1955, pp. 242-7). The least densely hydroxylated of these $1 s$ the $(0001)$ face of $\beta$-tridymite, which is covered with tertiary silica groups and has a surface silanol density of $4.6 \mathrm{E} 14 \mathrm{~cm}^{-2}$. The most densely hydroxylated is the surface of 8 -cristoba1ite which is covered with secondary sillca groups and has a surface silanol density of $7.85 E 14 \mathrm{~cm}^{-2}$.

The apparent change of the rate law for molecular deposition from approximately fifth to approximately first kinetic order at a certain dissolved sillca concentration may also be explained in terms of "adsorbed silica" density. At low MAS concentrations, the surface concentration of "adsorbed" silica is low, and the rate determining step in the overall reaction is the rearrangement and interlinkage of "adsorbed sil1ca" groups on the surface to form "bulk" solid AS. This process may be expected to involve several "adsorbed sillca" groups and, hence, the high apparent kinetic order. At high MAS concentrations, the density of adsorbed silica " groups on the surface is so large that the coordinated rearrangement and interlinkage of the "adsorbed silica" groups is no longer rate determining. Instead, the step

$$
\text { dissolved silica } \rightarrow \text { "adsorbed silica" }
$$


becomes rate determining and this reaction is first order in dissolved silica concentration.

The highest MAS concentration in Figure 3.11 is $0.4 \mathrm{~g} \mathrm{~L}^{-1}$ which is approximately equal to the threshold concentration that separates the fifth and first kinetic order domains. The largest amount of adsorbed silica measured, about $2 \mathrm{E} 14 \mathrm{SIO}_{2}$ units $\mathrm{cm}^{-2}$, Is equal to about half a monolayer: It is not possible to say whether or not the amount of "adsorbed sifica" continues to increase with increasing MAS concentration at higher MAS concentrations. It may be that at this concentration or slightly beyond it the surface structure reaches a limiting "saturated", state, and ceases to change further with increasing MAS concentration. In our analysis of the homogeneous nucleation data (see S3.10) we found no need to postulate a direct effect of dissolved silica concentration on the value of the surface tension. This is consistent with the hypothesis of a constant "ifmiting" surface structure throughout the range for which homogeneous nucleation data is avallable, but does not necessarily prove it to be true.

The small oligomer data reviewed in S2.10 thru 12 suggests that most of the "adsorbed silica" consists of secondary groups that are added on to the surface by "being inserted" into preexisting slloxane bridges. The addition of one such group to the surface involves the splitting off of one molecule of water. By a simple extension of the derivation in S3.11, we can demonstrate that the presence of salts in the solution will have approximately no effect on the free energy of the reaction

$$
\mathrm{MSA}+\text { "adsorbed } \mathrm{SiO}_{2} "+\mathrm{H}_{2} \mathrm{O}
$$

because the effect of the salt on the activity coefficient of MSA will approximately cancel out the effect of the salt on the activity of water. Therefore, at a constant MAS concentration (in units of grams of $\mathrm{SiO}_{2}$ per kilogram water) adding salts to the solution should not effect the surface density of "adsorbed silica" very much. This is why we chose to use $S_{a}$ Instead of $S$ in defining the function that describes the effect of concentration on the molecular deposition rate. 


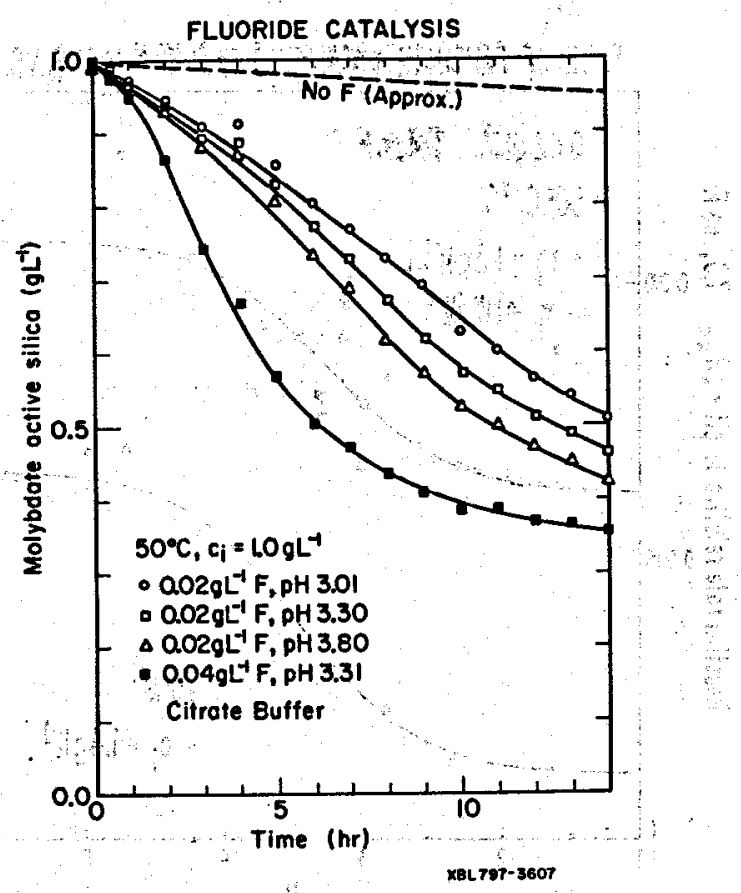

F1g. 3.12. The "No F" curve was estimated from another curve (not shown here) that was run at a higher $\mathrm{pH}$ by adjusting its time scale to make it correspond to $\mathrm{pH}=3.3$.

\section{S3.6 Catalysis by Fluoride}

Several sets of homogeneous nucleation experiments were run at 50 and $70^{\circ} \mathrm{C}$ with fluoride added to the solutions. $\mathrm{pH}$ values from $1.49^{\text {to }} 5.28$ and silica concentrations of $1.0,1.3$, and $1.4 \mathrm{~g} \mathrm{~L}^{-1}$ were employed. The duration of the experiments ranged from four to twelve hours. There were four, five, or six experiments per set.

The amount of fluoride added in most cases was $0.02 \mathrm{~g} \mathrm{~L}-1(1.05$. milifmolar). It was added in the form of $\mathrm{NaF}$. This value was routinely used because it is approximately equal to the maximum fluoride concentration that is observed in geothermal brines, and gives a reaction rate fast enough to work with conveniently. One experiment was run with $0.04 \mathrm{~g} \mathrm{~L}^{-1}$ added fluoride, and it confirmed our expectation that the rate of the fluoride catalyzed pathway is proportional to the fluoride concentration up to at least this value. Some "reference experiments" with the same temperatures and inftial MAS concentrations and comparable $\mathrm{pH}$ values but no added fluoride were also run. The results of these experiments were used to relate the rate of the fluoride catalyzed pathway to that of the base catalyzed pathway. 


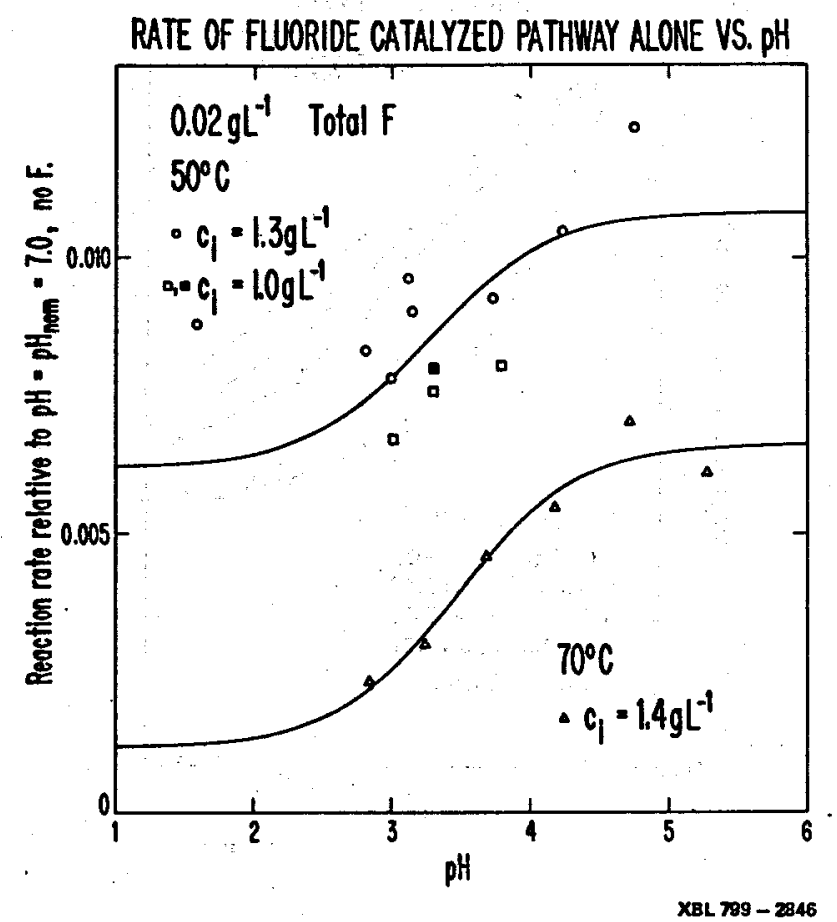

Fig. 3.13.

The results obtained in one of the sets in this series are presented in Figure 3.12. The results from another set are presented in tabular form in Table A3.7 in Appendix 3.4.

Average rate ratios relating pairs of curves were calculated as in the case of the $\mathrm{pH}$ dependence experiments (see 53.3). The single number obtained for each experiment in this way was the "rate of polymerization" under the conditions of that experiment divided by the "rate" under the conditions of another experiment in the same set. The assumption was made that the overall reaction rate could be separated into a fluoride catalyzed term, and a "base catalysis only" term. The latter was calculated for each fluoride added experiment using the results of the corresponding "reference experiment". It was then subtracted out of the overall rate determined for the given fluoride added experiment, leaving the rate of the fluoride catalyzed pathway alone. The rate of the fluoride catalyzed pathway was finally converted to a rate value relative to that of the base catalyzed pathway at $\mathrm{pH}=\mathrm{pH}_{\text {nom }}=7.0$. This procedure allowed data from different sets of experiments with different initial silica concentrations to be combined and analyzed together. The final results of this procedure are presented in Figure 3.13. 
There are three concelvable fluortde catalyzed mechanisms for siloxane bond formation. In the case of the dimerization of MSA to give disilicic acid (DSA), these would be

$$
\begin{aligned}
& \mathrm{MSA}+\mathrm{Si}(\mathrm{OH})_{4} \mathrm{FH}+\mathrm{DSA}+\mathrm{HF}+\mathrm{H}_{2} \mathrm{O}^{2} \\
& \mathrm{MSA}+\mathrm{Si}(\mathrm{OH})_{4} \mathrm{~F}^{-}+\mathrm{DSA}+\mathrm{F}^{-}+\mathrm{H}_{2} \mathrm{O}^{-}
\end{aligned}
$$

preceeded, in each case, by the formation of one or the other intermediate complex. (Alternatively, the complex in reactions (3.6.1a) and (3.6.2) could have the composition $\mathrm{SI}(\mathrm{OH})_{3} \mathrm{~F}$; this formula would give the same overall rate laws as the one used above.)

The rate of reaction by mechanism (3.6.1a) would be proportional to the concentration of $\mathrm{HF}$. At constant salinity, the rate via either mechanism (3.6.1b) or (3.6.2) would be approximately proportional to the concentration of $F^{-}$. These concentrations are related to the total concentration of $F$ by the dissociation equilibrium for $\mathrm{HF}$ :

$$
\begin{aligned}
\left(F^{-}\right) & =\left(F_{\text {tot }}\right) \alpha_{F} \\
(\mathrm{HF}) & =\left(F_{\text {tot }}\right)\left(1-\alpha_{F}\right)
\end{aligned}
$$

where all concentrations are in molal unfts, and

$$
\begin{aligned}
& \left(F_{\text {tot }}\right)=\left(F^{-}\right)+(H F) \\
& F=1 /\left(1+\left[H^{+}\right] \gamma_{F}-/ K_{H F}\right)
\end{aligned}
$$

To an adequate approximation, between 5 and $125^{\circ} \mathrm{C}$

$$
\log \mathrm{K}_{\mathrm{HF}}=-1.892+403.6 / \mathrm{T}+0.012465 \mathrm{~T}
$$

(This formula was fitted to the data of Naumov, Ryzhenko and Khodakovsk11, 1971, p. 239, as quoted by Kharaka and Barnes, 1973.) sisis:

As it turned out, two separate mechanisms, one with rate proportional to (HF) and another with rate proportional to ( $F^{-}$) had to be postulated to explain the data. That is to say, mechanism $(3.6 .1 \mathrm{a})$ and either one or both of (3.6.1b) and (3.6.2) occur. It was al so found that fncreasing the sa:1nity by adding $\mathrm{NaCl}$ while maintaining constant total fluoride and approximately constant pH had relatively 1Ittle effect on the rate of the fluoride catalyzed pathway. In other words, the rate of the fluoride catalyzed pathway does not seem to depend directly on the value of $\mathrm{F}\left(\mathrm{pH}, \mathrm{pH}_{\mathrm{nom}}\right)$. This is consistent with mechanism $(3.6 .1 \mathrm{~b})$, but inconsistent with mechanism $(3.6 .2)$. 
Therefore, the overal! rate of molecular deposition in the presence of fluoride, including the base catalyzed pathway, may be written as

$$
\begin{aligned}
& R_{\mathrm{md}}\left(\mathrm{g} \mathrm{SiO} \mathrm{cm}^{-2} \mathrm{~min}^{-1}\right) \\
& =\mathrm{f}_{\mathrm{f}}\left(\mathrm{S}_{\mathrm{a}}\right)\left(1-\mathrm{S}^{-1}\right) \mathrm{k}_{\mathrm{OH}}(\mathrm{T})\left[\mathrm{F}\left(\mathrm{pH}_{\mathrm{pH}} \mathrm{pH}_{\mathrm{nom}}\right)\right. \\
& \left.\quad+\mathrm{F}_{\mathrm{tot}}\left(\left(1-\alpha_{\mathrm{F}}\right) \mathrm{k}_{\mathrm{HF}}(\mathrm{T})+\alpha_{\mathrm{F}} \mathrm{k}_{\mathrm{F}}(\mathrm{T})\right)\right]
\end{aligned}
$$

where $k_{H F}(T)$ and $k_{F}-(T)$ are the apparent rate constants for the two fluoride catalyzed pathways. The values of the two rate constants at 50 and $70^{\circ} \mathrm{C}$ were determined by fitting the data in. Figure 3.13 as a function of $\alpha_{F}$. The fitted values of the sum of the rates of the fluoride catalyzed . pathways are presented as solid lines in Figure 3.13. Assuming molal concentration units for the fluoride species, the values obtained for the rate constants were

$$
\begin{array}{llll}
\text { at } 50^{\circ} \mathrm{C}, & \mathrm{k}_{\mathrm{HF}}=5.92 & \text { and } & \mathrm{k}_{\mathrm{F}}=10.3 \\
\text { at } 70^{\circ} \mathrm{c}, & \mathrm{kHF}_{\mathrm{HF}}=1.11 & \text { and } & \mathrm{k}_{\mathrm{F}}=6.32
\end{array}
$$

These values were then fitted as functions of temperature:

$$
\log k_{H F}(T)=-11.723+4039 / T
$$

$\log \mathrm{k}_{\mathrm{F}}(\mathrm{T})=-2.647+1183 / \mathrm{T}$

The rates of the fluoride catalyzed pathways decrease relative to the rate of the base catalyzed pathway with increasing temperature, but not in an absolute sense.

These formulas should be used with caution because of the uncertainty of the fitted values and the small temperature difference. In the 50 to $70^{\circ} \mathrm{C}$ range, the values of $k_{F^{-}}$calculated from $(3.6 .7 \mathrm{~b})$ are probably as reliable as most of the other fitted values in this report. However, the values of $k_{H F}$ calculated from $(3.6 .7 a)$ are at best semiquantitative, and should be used accordingly.

Practically speaking, when $F_{\text {tot }}=1 \mathrm{E}-3$ molal, the fluoride catalyzed mechanisms become dominant below about $\mathrm{pH} 4.8$. In the presence of $5 \mathrm{E}-5$ molal F, they become dominant below about $\mathrm{pH} 3.5$. This sets a natural limit to the degree that silica precipitation from geothermal brines may be inhibited by $\mathrm{pH}$ reduction alone. However, aluminum complexes with fluoride and blocks the fluoride catalyzed pathways. This is discussed in Section 3.15 . 


\section{S3.7 Nucleation Theory: The Lothe-Pound Factor}

A particle of critical nucleus size is actually in rapid motion: it diffuses through the water, and it executes rapid "jiggling motions" of both translational and rotational nature on a smaller scale of time and space. These degrees of freedom greatly decrease the free energy of formation of the critical nucleus. The classical Theory of Nucleation ignores these degrees of freedom, and that is its main deficiency. The Lothe-Pound Theory takes them into account. This is why we chose to employ the Lothe-Pound Theory. (For a detailed description of both theorles see the book by Abraham, 1974.)

The development of the Lothe-Pound theory starts out by writing the free energy of formation of a particle consisting of $n$ monomer units from a solution of given saturation ratio $S$ as:

$$
\begin{aligned}
F(n)=-k_{B} T \ln s+A(n) Y & \\
& -k_{B} T \text { ln } Q_{e x t}+k_{B} T \ln Q_{r e p}
\end{aligned}
$$

where $A(n)$ is the area of a spherical colloidal particle composed of $n$ monomer units and $\gamma$ is the surface tension.

The first two terms are simply the expression for the free energy which is employed in the classical Theory of Nucleation. The third term accounts for the free energy contribution of the six translational and rotational degrees of freedom of the particle as a whole. Qext is the corresponding "external" partition function. $Q_{\text {rep }}$ is the "replacement" partition function. It is analogous to Qext, but represents the contribution of the "external" degrees of freedom to the free energy of a particle of $n$ monomer units imbedded In solid AS rather than in aqueous solution. The corresponding free energy term is actually a correction to the first term. It accounts for the fact that the internal vibrational spectral density of a small particle of $\mathrm{SIO}_{2}$ differs from that of bulk AS in that the former is truncated at a wavelength equal to twice the particle diameter. Essentially, the fourth term subtracts out the contributions of the long wavelength bulk AS modes which the particle does not possess. (Note that its sign is opposite that of the third term).

The Lothe-Pound factor is defined as

$$
Q_{\text {LP }}=Q_{\text {ext }} / Q_{\text {rep }}
$$


Weres and Rice (1972) have demonstrated that the thermodynamic properties of licuid water may be well accounted for using a "cell model" of the liquid. The key assumption made by these authors is that, on the time scale of intermolecular (1.e., "lattice") vibrations, water molecules stay in more or less the same place and "jiggle" around in oscillatory translational and limited rotational ("librational") motions. This is due to the strength and highly directional character of the hydrogen bonds. The actual positions of the molecules and the pattern of hydrogen bonds connecting them change on a considerably longer ("diffusional") time scale. (This separation of time scales in water was first proposed by Eisenberg and Kauzmann, 1969, based on a careful interpretation of a variety of spectroscopic and other data.)

If the spectrum of molecular motions can be separated in this way, then so can the thermodynamic propertles. Put another way, the N-particle partition function of liquid water may be factored into two parts: a partition function that arises from the "lattice" modes, and a "configurational" partition function that reflects the number of ways that the $\mathrm{N}$ molecules and the hydrogen bonds between them may be arranged. Indeed, the configurational partition function is simply that number. The former, lattice mode, partition function is essentially that of an amorphous molecular solid. This is so because, on the time scale of th: "lattice" motions, 1iquid water behaves as though it were an amorphous solid.

Silicic acid oligomers and colloidal AS particles are hydrogen bonded to the water around them by way of their surface silanol groups, and diffuse far more slowly than do water molecules because of their much greater size and the large number of hydrogen bonds they engage in. Therefore, the basic assumption of Weres and Rice is even more appropriate in this case. Accepting it allows Qext to be separated into two factors: a lattice mode factor which accounts for the "jiggling around" of the AS particle imbedded in the aqueous medium and hydrogen bonded to it, and a configurational partition function which accounts for the different locations and orientations which the particle may have in the water. The configurational partition function may further be factored into two parts that are respectively equal to the number of positions and the number of orientations avallable to the particle in the water. Thus, we may write

$$
Q_{\text {ext }}=Q_{1 \text { at }} Q_{\text {pos }} Q_{\text {orien }}
$$


On closer examination. 1t is easy to see that the multiplicity of orientations need not be explicitly considered here. If Qorien is to be included in the definition of $Q_{e x t}$, an analogous factor must be included in the definition of $Q_{\text {rep }}$ as well, and the two will cancel out. Alternatively, one may argue on physical grounds that, because AS is amorphous and isotropic, all orientations of a spherical particle of it are physically Identical and this makes

\section{Qorien $=1$}

The value of $Q_{\text {pos }}$ is rigorously given by a simple extension of Raoult's Law:

... The number of positions within a given volume of water is equal to the total number of molecules within it.

If the water is salt free and the nucleation rate is to be expressed in units of (min $\left.\mathrm{kg} \mathrm{H}_{2} \mathrm{O}\right)^{-1}$, this number is simply the number of water molecules In a kilogram of water $=3.34 \mathrm{E} 25$. In a salt solution, it is approximately equal to the number of water molecules plus the number of salt 1ons per kilogram of water. However, this "salt correction" is so small that it may be Ignored for all practical purposes. (After all, other approximations introduce much larger errors into the final result.) Therefore, in the present system of units

$$
Q_{\text {pO5 }}=3.34 E 25
$$

$Q_{1 a t}$ is completely analogous in its physical significance to $Q_{r e p}$; the only difference is that the former applies to an AS particle imbedded in an aqueous medium, while the latter applies to an identical particle imbedded In solid AS. Simple dimensional considerations lead to the conclusion that

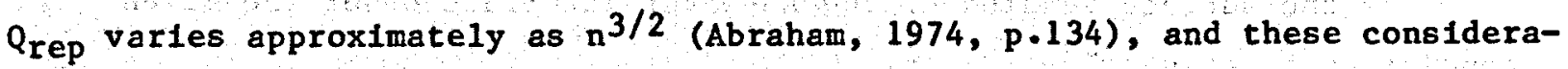
tions apply equally to $Q_{1 a t}$. Therefore, the ratio of the two is approximately independent of $n$. This ratio was evaluated approximately by statistical mechantcal methods for the case of $n=1$ (1.e, the MSA molecule) and found to be close to unity. It is, therefore, probably close to unity for all values of $n$. Combining all of the preceeding arguments:

$$
Q_{\mathrm{LP}}=3.34 \mathrm{E} 25
$$

The error in this value $1 \mathrm{~s}$ probably no more than about an order of magnitude. Because only the minus $1 / 4$ th or minus $1 / 3$ rd power of the value of $Q_{L P}$ is directly measurable physically (through its effects on the induction time and 
on the total surface area of the particles produced, respectively), (3.7.3) is, for most practical purposes, exact.

This is the factor by which classical nucleation theory underestimates the rate of nucleation in this system!

\section{S3.8 Nucleation Theory: Further Development}

The fact that $Q_{L P}$ is practically constant means that it disappears when (3.7.1) is differentiated in respect to $\mathrm{n}$ and in all analogous mathematical manipulations. In practice, this means that the only way that the final practical results of the Lothe-Pound Theory differ from those of the Classical Theory is that the calculated rate of nucleation is increased by a factor equal to $Q_{L P}$. This Section is a brief resume of the resulting further developments. It draws heavily on the exposition by Abraham (1974).

The steady state nucleation rate is

$$
I_{M}=Q_{L P} z R_{m d} \rho_{n} A^{*} \exp \left(-\Delta F^{*}\right)
$$

where

$\rho_{\mathrm{n}}=$ the number density of $\mathrm{SHO}_{2}$ units in solid AS $=2.21 \mathrm{E} 22 \mathrm{~cm}^{-3}$

$A^{*}=$ the surface area of the critical nucleus under the given condjtions $=4 \pi r * 2$

where

$r^{*}=$ the radius of the critical nucleus under the given conditions, as calculated from the Classical Theory of Nucleation

$$
=2 \gamma /\left(\rho_{n} k_{B} T \ln s\right)
$$

where

$=$ the surface tension of the AS-water interface under the given conditions in ergs $\mathrm{cm}^{-2}$

$\mathrm{k}_{\mathrm{B}}=$ the Boltzmann constant $=1.38054 \mathrm{E}-16$ ergs $\mathrm{K}^{-1}$

$\Delta F^{*}=$ the free energy barrier for homogeneous nucleation as calculated from the Classical Theory

$$
=\gamma A * / 3
$$

$Z$ = the "Zeldovich Factor", which accounts for the fact that only a fraction of the nuclei that reach critical nucleus size continue to grow beyond it (the rest "fall back"). Typically, its value is between 0.005 and 0.1 . 


$$
\begin{aligned}
z & =\left[-\partial^{2} \Delta_{F} / \partial_{n} *^{2} /\left(2 \pi k_{B} T\right)\right]^{1 / 2} \\
& =2 / 3\left[3 /\left(4 \pi \rho_{n} n *^{2}\right)\right]^{1 / 3}\left(Y / k_{B} T\right) 1 / 2 \\
\text { where } & \\
n^{*} & =\text { the number of } S 10_{2} \text { units in the crittcal nucleus } \\
& =4 \pi / 3 \rho_{n} r^{* 3}
\end{aligned}
$$

As noted, Eqn. (3.8.1) gives the steady state nucleation rate. At very short times after initiating the reaction, the nucleation rate is less than this because a steady state population of oligomers of near critical nucleus size has not yet been established. There are two ways to deal with this effect. The mathematically exact way is the so called "multistate kinetics" method, in which the calculation of the time dependent flux of particles past critical nucleus size is treated as a diffusion problem in particle size space. The particles are assumed to randomly diffuse through size classes defined by the number of monomers that the particles belonging to them contain: $n^{*}-2, n^{*}-1, n^{*}, n^{*}+1, n^{*}+2$, etc. The number of particles In each of these size classes is calculated as a function of time by numerically integrating the appropriate kinetic equations. See Abraham (1969) and Abraham (1974, pp. 91-101) for an example of this method.

The other way is to use an approximate analytic solution for this time dependent diffusion problem. Many such derivations yield the following approximate expression for the time dependent nucleation rate (Abraham, 1974, p. 99):

$$
I_{N}(t)=I_{N}\left[1-\exp \left(-t / \tau_{c}\right)\right]
$$

where

$t=$ the time from the start of the reaction $c=$ the "time constant"

The nucleation rate as given by Eqn. (3.8.4) is to be understood as the rate at which particles of size $n^{*}+0.5 / 2$ are produced. Th1s is approximately the size at which the growth of the particles ceases to be diffusionlike and becomes effectively one directional and irreversible (see Feder et al., 1966, pp. 132-6).

Abraham (op. c1t.) found that the following expression for $\tau$, which was originally derived by Collins (1955), gave the best agreement with his own mathematically exact "multistate kinetics" results:

$$
\tau_{c}=1 /\left(4 \rho_{n} R_{m d^{A}} z^{2}\right)
$$




\section{S3.9 Homogeneous Nucleation: Experimental Results}

The kinetics of silica polymerization by homogeneous nucleation in low salinity'(i.e., "buffer only") media were extensively studied experimentally. The best of this data is compiled In Figures 3.14 to 18 and in Tables A3.8 to 12 in Appendix 3.4 .

These experiments were performed at various $\mathrm{pH}$ values between about 5.5 and 7.5." In each case the $\mathrm{pH}$ value was chosen so as to make the reaction run its course in a convenfent length of time, if at all possible. In a few cases that correspond to the lower Initial concentration values at the given temperature this was not possible, and the reaction was allowed to run for as long as needed to take it to completion (up to several days).

The actual unmodified MAS versus time data is presented in Tables A3.8 to 12 in $\mathrm{A} 3.4$. Most of the $50^{\circ} \mathrm{C}$ nucleation data is presented in unmodified form in Figure 3.18 as well.

The different $\mathrm{pH}$ values of the various experiments make the unmodified data hard to compare in a meaningful way. Therefore, the data is presented in Figures 3.14 to 17 with the (logarithmic) time scale of each curve shifted so as to mal:e it approximate the results that would have been obtained if each experiment had been run at $\mathrm{pH}=\mathrm{pH}_{\text {nom }}=7.0$. Specifically, the time scales in Figures 3.14 through 17 are related to the actual experimental time values by the relation:

$$
\log t_{\text {figure }}=\log t_{\text {actual }}+\log \mathrm{f}_{\mathrm{pH}}^{\circ}\left(\mathrm{pH}_{\text {nom }}\right) .
$$

This shift in the time scale is different for each individual curve. Thus, curves with $\mathrm{pH}_{\text {nom }}>7.0$ were shifted to the right ("slowed down".) and curves with $\mathrm{pH}_{\text {nom }}<7.0$ were shifted to the left ("speeded up"). (The value of $\mathrm{pH}_{\text {nom }}$ for each curve is presented in Tables A.3.8 thru 12, and $f^{\prime}{ }_{\mathrm{pH}}\left(\mathrm{pH}_{\mathrm{nom}}\right)$ may be evaluated using either Figure 3.4 or Table $\mathrm{A} 3.1$, if so desired.)

These time shifts are only approximate. First, the effect of varying $\mathrm{pH}$ on the value of the surface tension was 1gnored. Second, the value of the shift in $\log \mathrm{t}$ should have been $\log \mathrm{F}\left(\mathrm{pH}, \mathrm{pH}_{\mathrm{nom}}\right)$. However, this would have made very little difference because in most cases included in these Figures $\mathrm{pH}$ is not much different from $\mathrm{pH}_{\text {nom. }}$. 


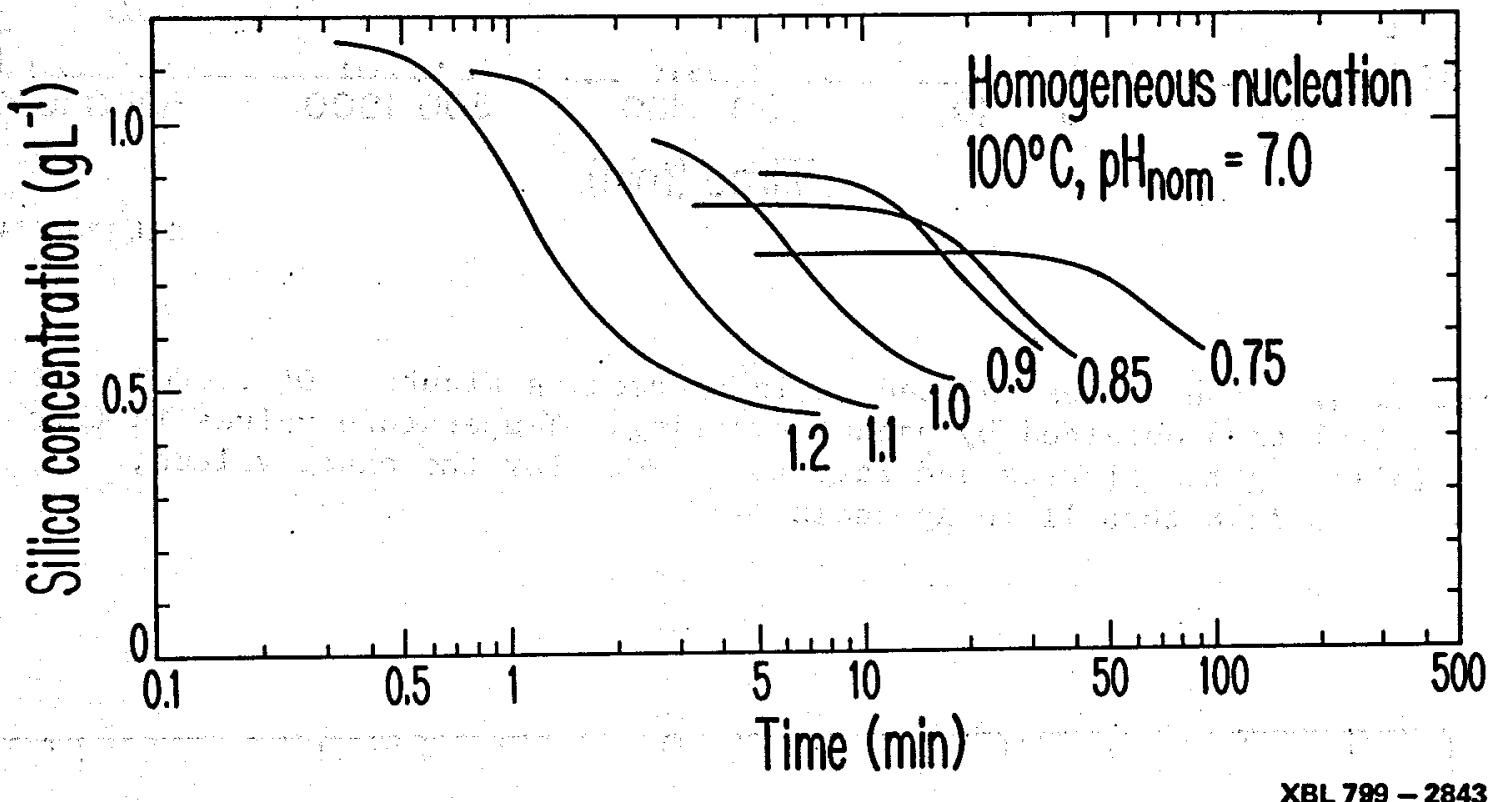

Fig. 3.14. Each curve in this Figure shifted along the $10 \mathrm{~g} t$ axis as discussed in the text. The curves in this figure are continuous because they were generated on the continuous flow kinetic system. 


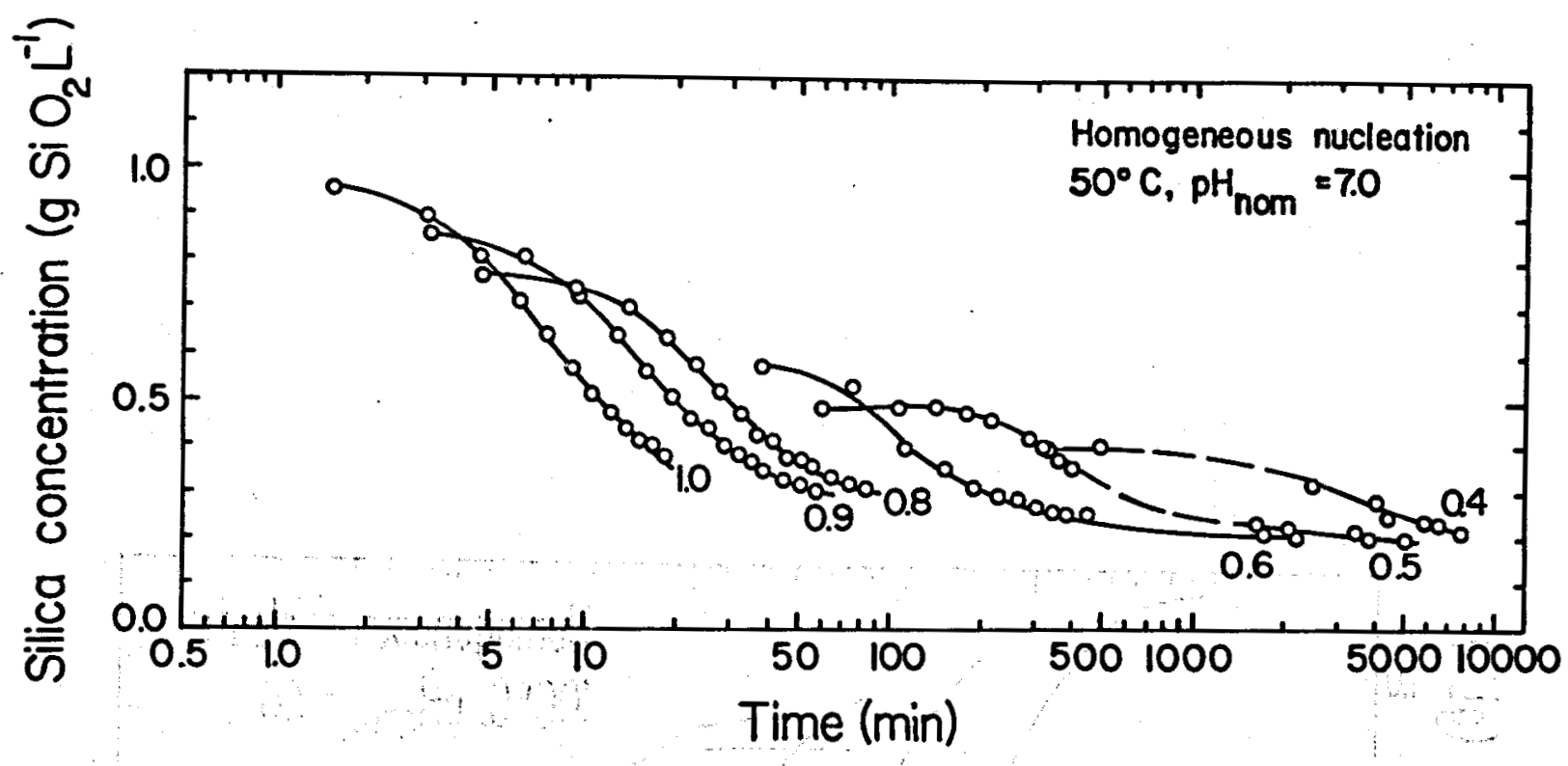

XBL $7812-13430$

Fig. 3.15. Time scales shifted as in preceeding Figure. Discrute points arc actual data obtained by manual sampling. Temperature values in this and following two Figures accurate to $\pm 1^{\circ} \mathrm{C}$. For the exact values, see lables A3.8 thru 11 in Appendix $3 . \overline{4}$.

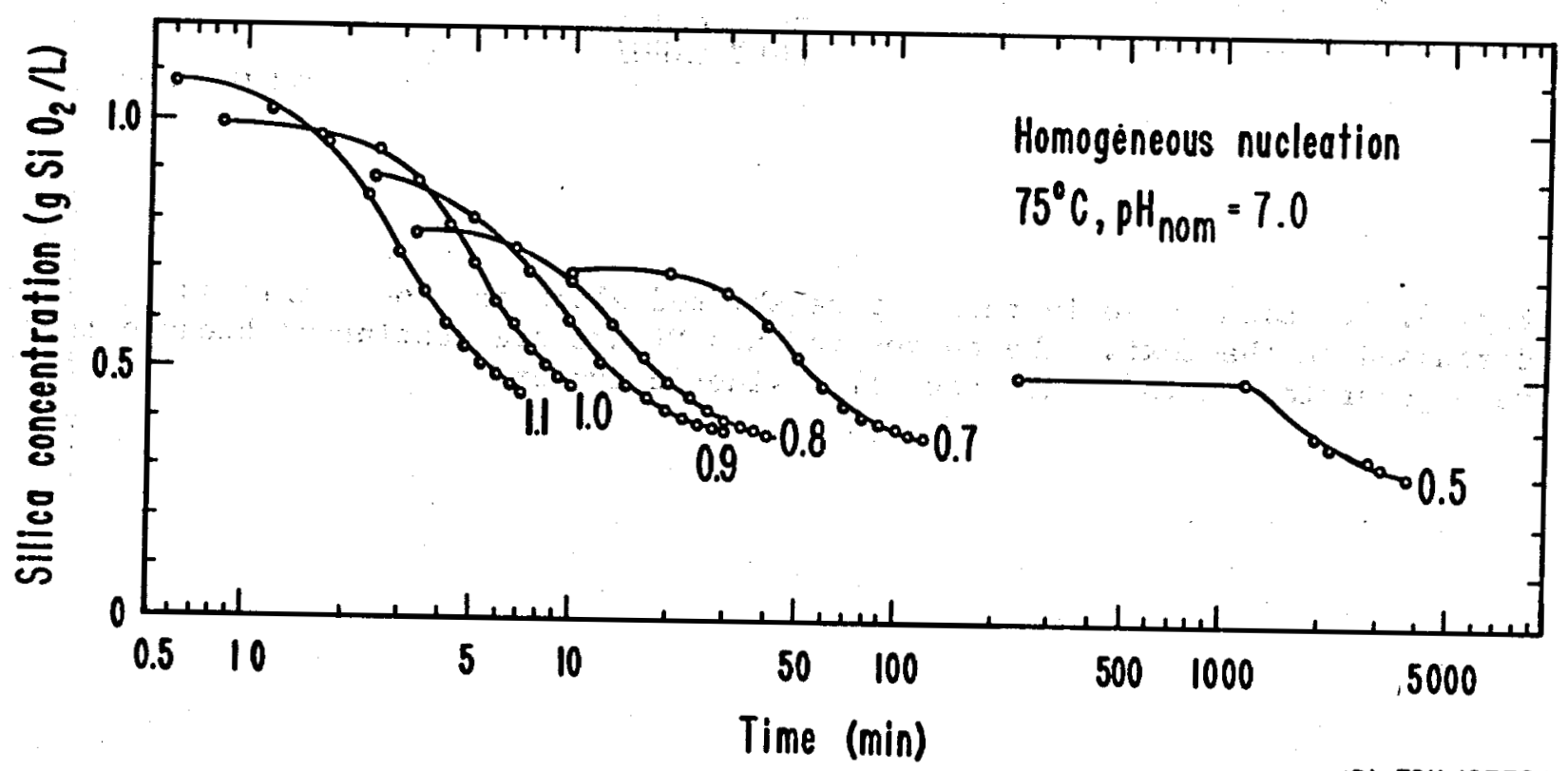

Fig. 3.16. Time scales shifted as in preceeding rigures. 


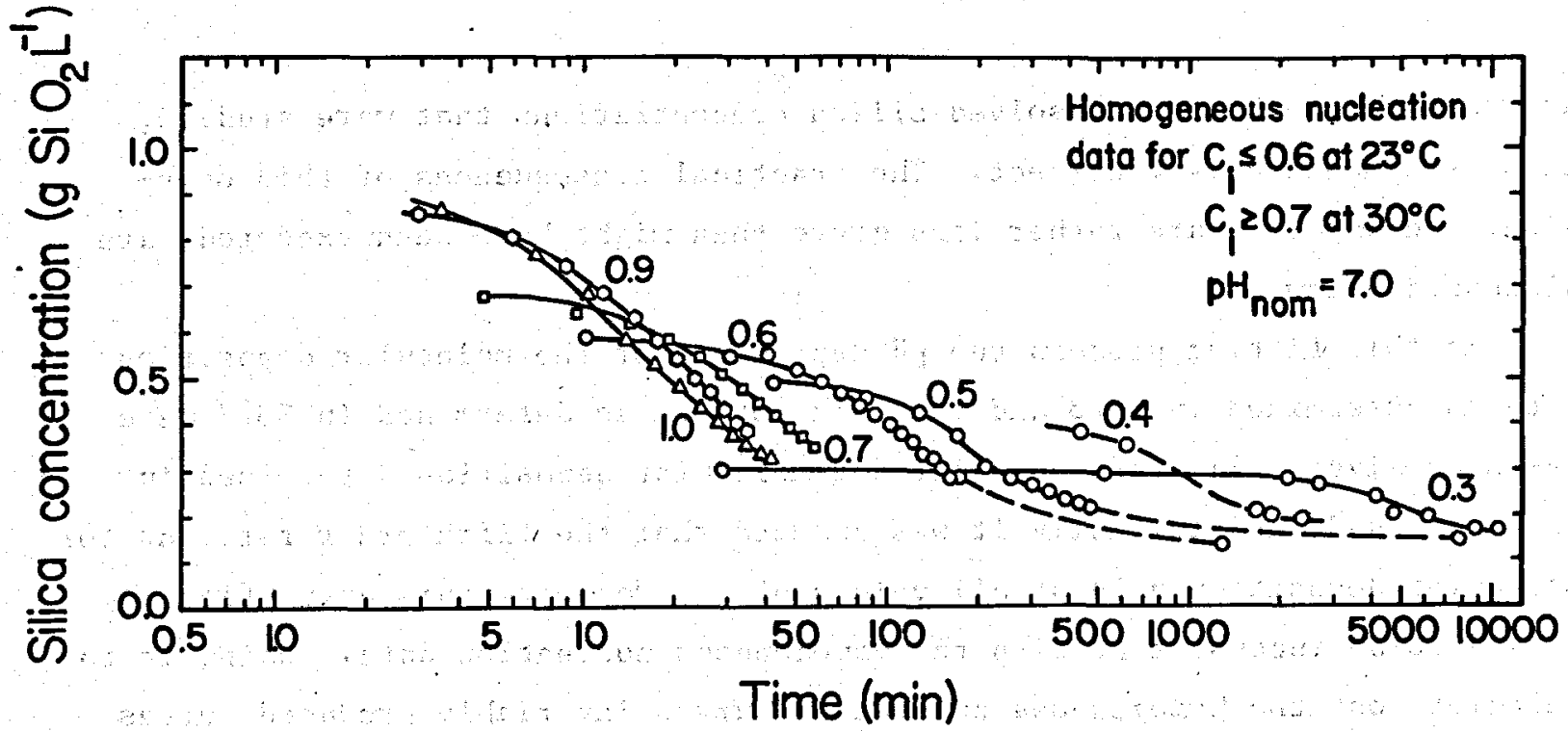

XBL 78R-13429A

Fig. 3.17. Time scales shifted as in preceeding Figures.

\section{S3.10 Homogeneous Nucleation: Data Reduction and the Surface Tension}

The homogeneous nucleation data was analyzed and reduced by "fitting" It using the theoretical formalism presented in 53.7 and 8 . A computer code that is able to numerically model the processes of homogeneous nucleation and colloid growth was used for this purpose. This code is called SILNUC and it is discussed in detall and documented in Chapter 6 of this report.

As originally written, SILNUC used the mathematicaily exact "multistate kinetics" method to model the homogeneous nucleation process. Later, the approximate "time constant" model (Eqns - 3.8.4 and 5) was added as an option. As it turned out, the two mathematical models give essent 1 ally 1 dentical results, and the more complicated and cumbersome "multistate kinetics" algorithm was ultimately taken out of SILNUC (more on this below).

The theoretical model embodied in sILNUC assumes that the total concentration of silica oligomers ( $1 . e .$, dimers, trimers, etc.) is always negligible relative to the concentration of MSA. Therefore, as far as the model embodied in SILNUC is concerned,

molybdate active bilica = monomeric s1lica

and the latter term will be used in discussing the theoretical results. 
At least at the higher dissolved silica concentrations that were studied, tnis is demonstrably incorrect. The practical consequences of this omission, and why they are rather less grave than might have been expected, are discussed below.

In this fitting process the $\mathrm{pH}$ dependence of the molecular deposition rate as determined in S3.3 and the value of $Q_{L P}$ as determined in 53.7 were taken as given. The rate constant for molecular deposition determined in S3.4 was used, and intially it was assumed that the fifth order rate law for molecular deposition held at all values of $S$. However, this assunption proved to be inconsistent with the homogeneous nucleation data. Using it to calculate out the howogeneous nucleation curves invariably produced curves that were "too flat"; i.e., the maximum (negative) slope of the curve of MAS versus loo $t$ was always too small. It was soon found that this deviation could be corrected by assuning that the variation of deposition rate with saturation ratio was linear above a certain value. This "threshold value" $s_{t}$ was estimated by trial and error for 50,75 and $100^{\circ} \mathrm{C}$ by varying it until the overall best "shape fit" was obtained for all of the homogeneous nucleation data at each temperature. These "best values" are approximately given by Eqn. $(3.4 \cdot 7 c)$.

It was found that the homogeneous nucleation data obtained at 23 and $30^{\circ} \mathrm{C}$ could not be well fitted using values of $S_{t}$ that could be extrapolated from the nigher tenperature values in any reasonable way. This bespeaks a fundamental change of mechanism at the lowest temperatures. This change is probably due to the much higher $S$ values encountered at the lower temperatures (at $30^{\circ} \mathrm{C}, 1.0 \mathrm{~g} \cdot \mathrm{L}^{-1}$ corresponds to $\mathrm{S}=7.8$ ). Very 1 ikely, the concentration of oligowers is large at these lowest temperatures. Also, the basic theoretical model of the nucleation process employed probably fails to some extent because of the very small critical nucleus size. Because of this, attempts to fit the 23 and $30^{\circ} \mathrm{C}$ data were abandoned, and all further effort was restricted to the higher three temperatures.

One aspect of this change and/or fallure that is directly evident in Fig. 3.17 is that, at the three highest initial concentrations, the course of tne reaction is almost unaffected by the value of the initial concentration. This suggests that nucleation is no longer the rate limiting step under these conditions. 
Once the functions that determine the rate of molecular deposition and the Lothe-Pound factor are known, all that is needed to completely spectfy the homogeneous nucleation process is a function that gives the value of the surface tension under any given conditions. The remainder of the homogeneous nucleation data reduction procedure essentially consisted of varying a trial function for the surface tension until an overall optimal fit was obtained.

Changing the value of the surface tension generally shifts the calculated curve along the $\log t$ axis without changing its shape. (An exception to this occurs when the trial value of the surface tension is clearly too low, in which case the shape of the curve is distorted in a characteristic manner.) For moderate changes in the value of $\gamma$, the shift in the time scale may be accurately estimated using the approximate but very good relationship

$$
\frac{\Delta \log \tau}{\Delta \gamma^{3}}=4 \pi /\left(3 \times 2.302 k_{B} T\right)\left(k_{B} T \rho_{n} \operatorname{lnS}\right)^{-2}
$$

where $\tau$ is the time at which the MAS concentration is equal to any given value. It has the same mathematical properties as the "Induction time" discussed in 52.18 . Eqn. $(3.10 .1)$ may be derived using $(3.8 .1),(3.8 .2)$ and $(2.18 .4)$.

In practice, we calculated a curve for each $c_{1}$ and $T$ using an estimated value of $\gamma$, and then graphically estimated the shift along the $\log t$ axis that would make the calculated curve approximately colncide with the corresponding experimental curve. Eqn. (3.10.1) was then used to determine an "empirical"

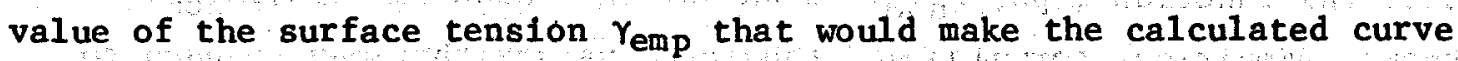
approximately coincide with the corresponding experimental curve. The values of Yemp were then fitted using a trial function to represent $Y$.

The trial function that was used is

$$
=\mathrm{H}_{Y}-\mathrm{TS}_{\gamma}-2.302 \mathrm{n}_{\mathrm{O}} \mathrm{k}_{\mathrm{B}} \mathrm{T} I\left(\mathrm{pH}, \mathrm{pH}_{\mathrm{nom}}\right)
$$

where

$\mathrm{H}_{Y}=$ the surface enthalpy in ergs $\mathrm{cm}^{-2}$

$S_{\gamma}=$ the surface entropy in ergs $\mathrm{cm}^{-2} \mathrm{~K}^{-1}$

$n_{0}=$ the surface density of lonizable surface sites. 


$$
\begin{aligned}
I\left(\mathrm{pH}_{\mathrm{pH}}\right) & =\int_{-\infty}^{\mathrm{pH}}\left[0.45 \mathrm{f}\left(\mathrm{pH}^{\circ}\right)+0.55 \mathrm{f}\left(\mathrm{pH}_{\mathrm{nom}}\left(\mathrm{pH}^{\circ}\right)\right)\right] \mathrm{d} \mathrm{pH}^{-} \\
& =0.118913 \int_{-\infty}^{\mathrm{pH}} \mathrm{F}\left(\mathrm{pH}^{\prime}, \mathrm{pH}_{\mathrm{nom}}\left(\mathrm{pH}^{\prime}\right)\right) \mathrm{d} \mathrm{pH}^{e}
\end{aligned}
$$

The integrand in (3.10.3) is equal to the fraction of lonizable surface sites that are actually lonized at the given values of $\mathrm{pH}$ and $\mathrm{pH}_{\mathrm{nom}} \cdot$ (See the discussion and definitions in S3.3.)

The first two terms in (3.10.2) give the values of $\gamma$ at the given temperature in the hypothetical (but closely approachable) state of no surface ionization. The third term corrects the value of $\gamma$ for the effect of surface ionization. It is easy to derive it from the Gibbs relation, which describes the effect of an arbitrary isothernal adsorption process on $\gamma$ :

$d \gamma=-\Gamma d \mu$

where

$\Gamma=$ the surface density of the adsorbed species

$\mu=$ the chemical potential of the adsorbed species in solution

(See, for exanple, the discussion by Adamson, 1976, pp.70-71.)

Because the function $\mathrm{F}\left(\mathrm{pH}, \mathrm{pH}_{\mathrm{nom}}\right)$ is known, the value of $\mathrm{I}\left(\mathrm{pH}, \mathrm{pH}_{\mathrm{nom}}\right)$ is also known. (Empirical foruulas and tabulated values that may be used to calculate the value of $I\left(\mathrm{pH}_{,} \mathrm{pH}_{\mathrm{nom}}\right)$ are presented in Appendix 3.1.) .

Therefore, the only unknowns in expression $(3.10 .2)$ are $\mathrm{H}_{\gamma},{ }^{S_{\gamma}}$ and $n_{0}$. These three parameters were determined by performing a multiple linear reg ression on the array of $\gamma_{\text {enp }}$ values deternined for each of the curves included in Figure 3.14 to 17. This fitting process was executed using values of $\mathrm{I}\left(\mathrm{pH}, \mathrm{pH}_{\mathrm{nom}}\right)$ evaluated with $\mathrm{pil}$ set equal to $\mathrm{pH}_{\mathrm{nom}}$ - Strictly speaking, this was an error, but only a minor one, in that $\mathrm{pH}$ and $\mathrm{pH}_{\text {nom }}$ differed little in most cases. The fitting process is summarized in Table 3.1.

It proved Impossible to fit a11 eighteen $\gamma_{\text {emp }}$ values well using (3.10.2); at each temperature, the one or two $\gamma_{\text {emp }}$ values that correspond to the lowest initial concentrations are too low to be fitted together with 
COMPARISON OF EXPERIMENTAL

AND CALCULATED CURVES

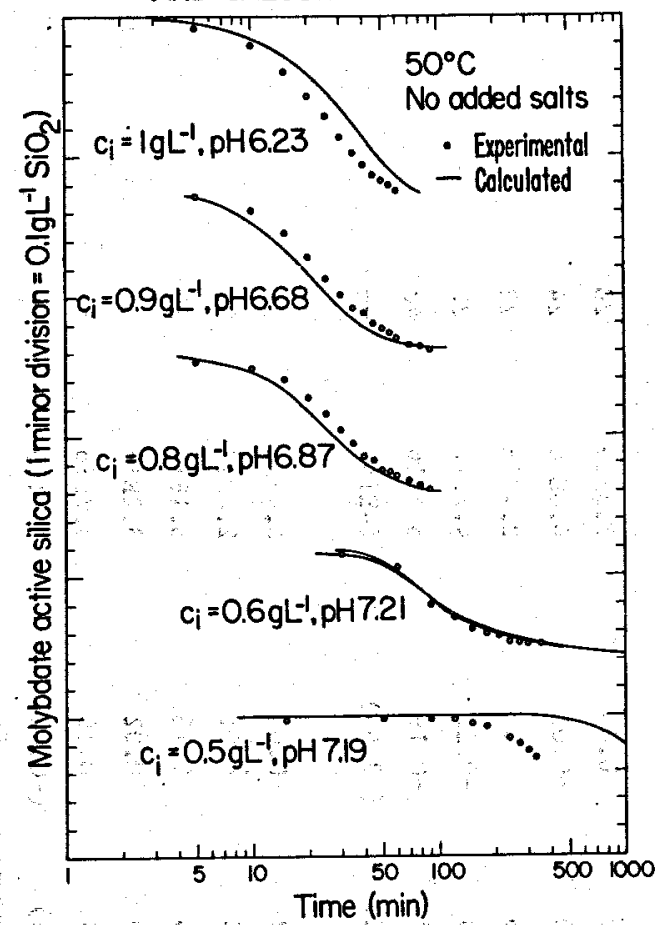

XBL799-2965

Fig. 3.18. Solid curves calculated by SILNUC. MAS concentration scale shifted by $0.5 \mathrm{~g} \mathrm{~L}^{-1}$ between curves. The light solid curve for $c_{1}=0.6$ was calcilated using the "multistate kinetics" algorithm (see text).

that tha critical nucleus has the geometric properties of a sphere and a well defined surface seems to work very well indeed. This is because the theory Implicitly averages over all possible clusters containing $n$ * monomer units to obtain the free energy of "the" critical nucleus. This implicit averaging process imposes rigorous spherical symmetry on the "average" critical nucleus that comes out of it; regardless of what assorted odd shapes the actual nucle1 of "critical" size may have. All this suggests that the theory of homogeneous nucleation and the concept of surface tension itself may be rather more powerful and widely applicable than has been hitherto believed.

Makrides et al. (1978) estimated the value of $Y$ at $95^{\circ} \mathrm{C}$ from their own homogeneous nucleation data and obtained the value 45 ergs $\mathrm{cm}^{-2}$." (Their approach was completely different from the one taken here.) The value of $\gamma$ at room temperature was determined from the emplrical relationship between the radius and solubility of colloldal AS particles by Alexander (1957) and Iler $(1979, \mathrm{pP} .55-6)$. The former obtained the value $46 \mathrm{ergs} \mathrm{cm}^{-2}$ and the latter obtained values of 46 and 54 ergs $\mathrm{cm}^{-2}$ using colloidal silica sols prepared In different ways. The solubility method has nothing at all to do with homogeneous nucleation and is largely free of ambiguities of interpretation. 
Table 3.1

Fitting of Homogeneous Nucleation Data

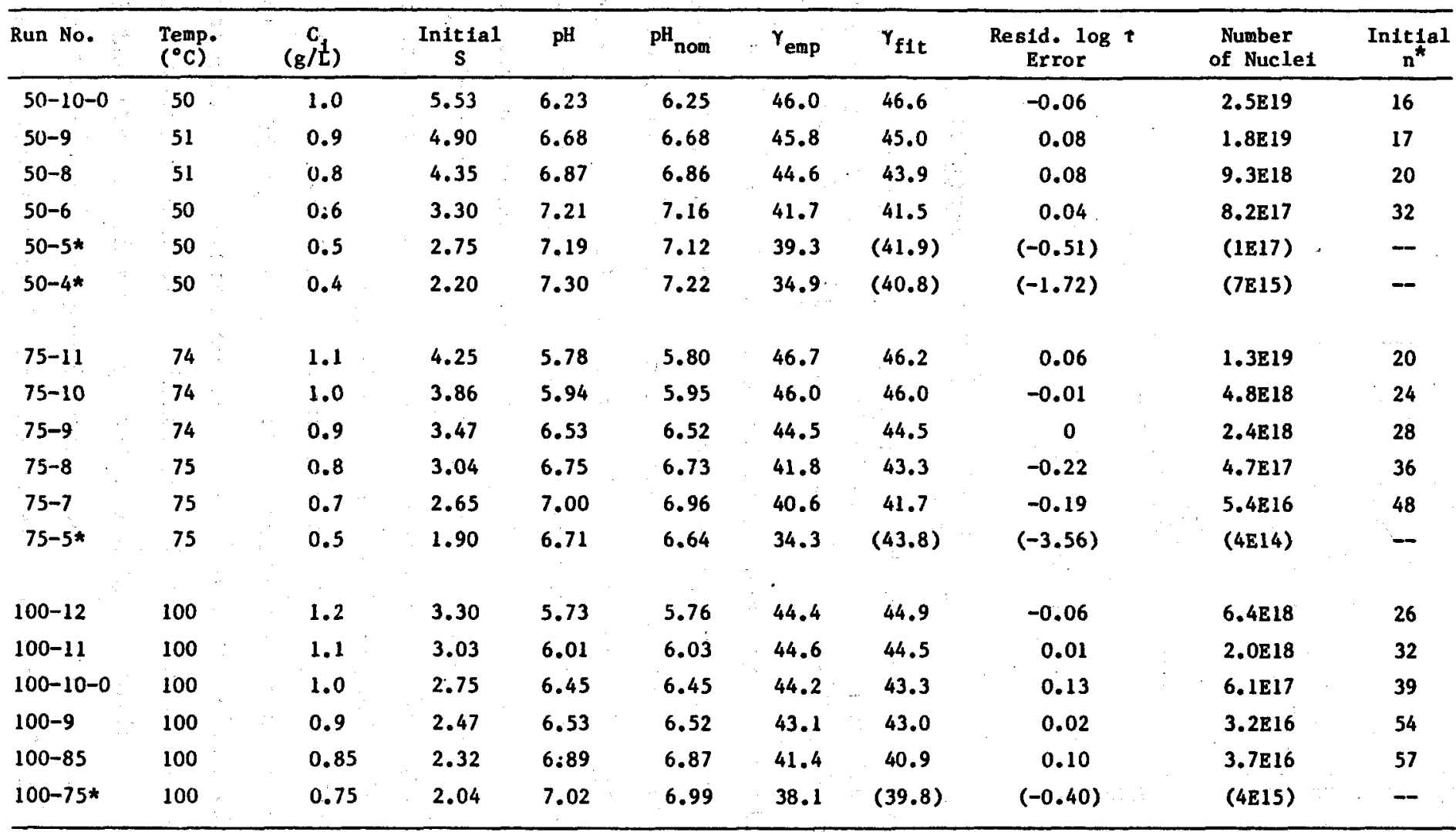

*Dominated by heterogeneous nucleation; data not fitted. In these cases $\gamma$ fit values calculated using the parameters given in the text, even though these particular points were not fitted. The residual log $\tau$ error values here, as elsewhere, approximately reflect the discrepancy between the experimental data and the homogeneous nucleation model. The "number of nuclei" values for the points not fitted were estimated from the experimental data.

The number of nuclei and $n^{*}$ values for the fitted curves were calculated using $\gamma_{f 1 t}$ as the value of the surface tension. 
Thus, the solubility derived values truly constitute independent confirmation of the values determined from homogeneous nucleation data.

The possible Importance of oligomer formation was estimated after the fact by estimating the dimer and trimer concentrations using the approximate empirical formulas (2.10.1 and 2).: Over the range of the data that was fitted with SILNUC, these ol1gomers never accounted for more than about $11 \%$ of the total dissolved silica and usually less. At these low concentrations, the only important effect that oligomer formation can have is that it reduces the monomer concentration and, thereby, the saturation ratio, and this slows down nucleation.

In a numerical sense, a change in the value of the saturation ratio can always be offset by a corresponding change in the value of the surface tension to give the same nucleation rate. The change in the surface tension needed to compensate for oligomer formation was calculated throughout the range of fitted data. It turned out that, In all cases considered, reducing the value of the surface tension by $4.3 \pm 0.3 \%$ compensated for the formation of oligomers.

It would have been easy to incorporate oligomer formation into SILNUC and to modify the surface tension values, but doing this would have had little or no effect on the calculated results. Therefore, we chose not to do it. However, if and when reliable data regarding oligomer concentrations become available, dolng so might become worthwhile.

All told, we believe our formula for $\gamma$ to be accurate to about \pm 3 ergs $\mathrm{cm}^{-2}$ in an absolute sense. Most of this uncertainty arises from our having assumed no oligomer formation and a constant, known value of the Lothe-Pound factor.

The individual values obtained for the three fitting parameters are less reliable because of the possibility of compensating errors. Nonetheless, they are reasonable. (See the discussion of $n_{0}$ in S3.5.),

The numbers of heteronuclei estimated for the low initial concentration experiments are not consistent from experiment to experiment, but this is only to have been expected in $1 \mathrm{lght}$ of the notoriously 1diosyncratic and poorly reproducible nature of the heterogeneous nucleation process. The data only allow us to suggest very approximate criteria for identifying cases in which heterogeneous nucleation is 1ikely to be dominant. One indication is 
that the predicted value of $\mathrm{n}^{*}$ is greater than about 60 . Another $1 \mathrm{~s}$ that the time required for the reaction to run halfway to completion under the given conditions; either as determined experimentally or as predicted theoretically, is greater than about $2 / \mathrm{F}\left(\mathrm{pH}, \mathrm{pH}_{\text {nom }}\right)$ hours, with the value of $\mathrm{F}$ evaluated for the conditions of interest. These estimates are, of course, based on the results of experiments that were performed under reasonably clean laboratory conditions. In actual geothermal brines, heterogeneous nucleation may become dominant at a somewhat higher initial concentration because of the greater number of potential heteronuclel present. Note, however, that the induction time for heterogeneous nucleation varies as only the minus one-third power of the number of hetronuclei (see Eqn. (2.18.4)); this greatly reduces the practical impact of possible variations in the number of heteronuclei insofar as making predictions is concerned.

\section{S3.11 The Solubility of Silica in Salt Solutions}

Fournier and Rowe (1977) give the following empirical formula for the solubility of AS in pure water under its own vapor pressure between 100 and $250^{\circ} \mathrm{C}$

$$
\log c_{0}(0)=-731 / T+1.52
$$

Because of very slow equilibration rates at lower temperatures, there is little reliable solubility data below $100^{\circ} \mathrm{C}$. However, (3.11.1) does appear to give results that are consistent with such data as is available, and it was used throughout the present work.

The salts in the brine also effect the solubility of silica. Because no usable data on this effect could be found in the literature, we estimated it theoretically.

The effect of dissolved salts on the activity of water has been experimentally determined for many solution compositions. For a solution containing one dissolved salt, this effect is given by the equation:

$$
\ln a_{w}=-0.018 \vee \mathrm{m} \phi
$$

where

$$
v=\text { the number of lons per nole of salt }
$$


$m=$ the molal concentration of the salt

$\phi=$ the practical osmotic coefficient

In the case of solutions of $1-1$ electrolytes, the practical osmotic coefficient is between 0.9 and 1.0 over large range of temperature and concentration. This means that the simple colligative effect upon the activity of water is the dominant one; $1 . e .$, the activity of water is decreased because the presence of the salt lons causes the water 1 tself to be diluted in the solution. Translated into statistical mechanical terms, the number of sites that water molecules may occupy within that amount of solution which contalns one $\mathrm{kg}$ of water is increased by the number of solute ions added. Other effects, which are manifested by the deviation of the value of the practical osmotic coefficient from unity, are much less important.

Under these circumstances, the effect of the added salt upon the activity coefficient of a "water-like" small solute like MSA is probably about the same as the effect upon the activity of water. This is so because the "dilution effect" will be exactly the same in the case of a molecule of MSA as in the case of water, and the much smaller residual effects will also probably be comparable.

The dissolution of solid silica is actually a hydration reaction: $\mathrm{SiO}_{2}$ (solid) $+2 \mathrm{H}_{2} \mathrm{O} \rightarrow \mathrm{SI}(\mathrm{OH})_{4}$

Two water molecules appear on the LHS, and one MSA molecule appears on the RHS. Therefore, in a salt solution, the lower activity of water reduces the equilibrium solubility by a factor of $a_{w}{ }^{2}$, while the lower activity coefficlent of MSA Increases it by a factor of approximately $a_{w}$. The net effect is

$c_{0}(m)=c_{0}(0) a_{w}$

where $c_{0}(m)$ is the solubility in a salt solution of molality to at the given temperature and $\mathrm{pH}$.

Both (3.11.1) and (3.11.4) Ignore that fraction of the dissolved silica that is in Ionic form. Therefore, the values of $c_{0}$ given by them include the equilibrium concentrations of MSA and other uncharged spectes only.

Eqn. (3.11.4) is true for all forms of solid silica. Nate, however, that th may not be accurate if the solution in question contains a substantial concentration of bi- or higher valent lons, as in this case the value of the osmotic coefficlent will no longer be approximately unity: Fortunately, this is very rarely the case with geothermal brines. 


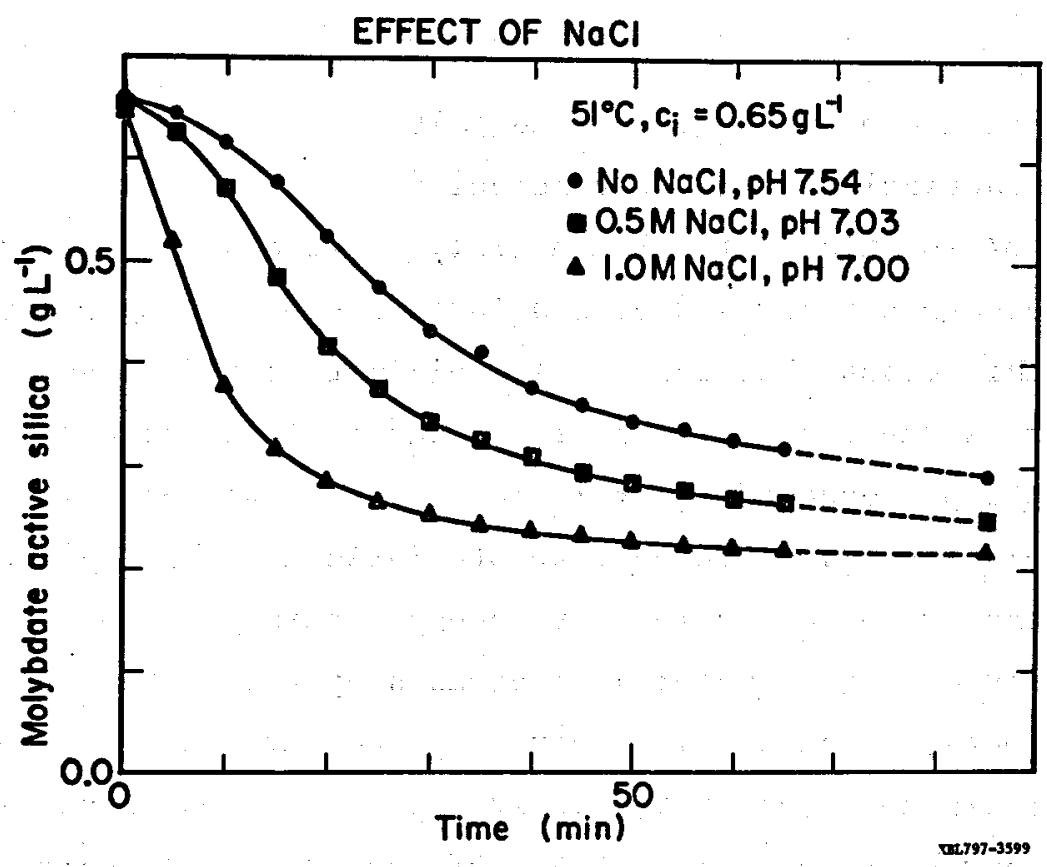

Fi8. 3.19. Salt concentrations given in units of moles $L^{-1}$ referred to room temperature (see $\$ 3.2$ ).

\section{S3.12 The kffect of Added Sodium Chloride}

Dissolved salts effect the rate of molecular deposition, the value of the surface tension, and the solubility of silica. Unfortunately, their effect on the rate of molecular deposition could not conveniently be studied in isolation with colloid added experiments because adding salts usually caused the collold to coagulate. Therefore, only experimental data of the homogeneous nucleation type could be used to study the effects of added salts.

Figure 3.19 shows the results of a typical "salt added" homogeneous nucleation experiment. The three runs included in it were formulated and run at the same time so as to minimize the effects of random variations. Altogether, twelve sets of three runs like this were generated, three each at 30 , 50,75 and $100^{\circ} \mathrm{C}$. The data from the set presented in Figure 3.19 and five other sets are presented in tabular form in Tables A3.13 to 15 in Appendix 3.4. A seventh set is presented in Figure 3.20.

The accelerating effect of the added salt is obvious from Figure 3.19, despite the fact that the reference (i.e., no NaCl) curve was run at a higher pH. 
The data analysis began in the same way as in $\$ 3.3$ and 53.6 . The raw data was graphed, and then converted into tabular data in the form of time as a function of concentration by estimating from the graph the time at, which the concentration of MAS was equal to a given value. Ratios of these time values were calculated, erratic values deleted, and the rest averaged to give average "relative rate" values. For example, the relative rate values calculated for the $0,0.5$ and $1.0 \mathrm{M}$ curves in Figure 3.19 were $1,1.62$, and 4.03, respectively.

Flnally, the empirical relative rate values were compared with theoretical ones calculated for the same set of conditions. In this context, the rate $(R)$ can be operationally defined as the reciprocal of $\tau$, the time that it takes for the concentration of MAS to drop to a given value.

Therefore, to a very good approximation

$R=1 / \tau=C F\left(p H, p_{n o m}\right)\left[Z A^{*} Q_{L P} \exp \left(-\Delta F^{*} /\left(k_{B} T\right)\right)\right]^{1 / 4}$

where $\mathrm{C}$ is a constant that depends on the "reference concentration" assumed in the definition of $t$. (Eqn. (3.12.1) is simply another way of writing $(2.18 .4)$.)

The theoretical relative rates are then simply the ratios of the values of $R$ for each of the curves in the given set calculated using (3.12.1). Note, however, that $Q_{L P}$ is constant and that the value of $\left(\mathrm{ZA}^{*}\right)^{1 / 4}$ varies but little between the three curves in each set. (It was found the $A^{*}$ decreases and $Z$ increases with increasing salinity 1 n such a way that the changes approx Imately cancel.) Therefore, to a good approximation, the ratio of rates for two curves in a given set may be calculated as

$$
\begin{aligned}
& R_{2} / R_{1}=\tau_{1} / \tau_{2} \\
& =F\left(\mathrm{pH}_{2}, \mathrm{pH}_{\mathrm{nom}, 2}\right) / \mathrm{F}\left(\mathrm{pH}_{1}, \mathrm{pH}_{\mathrm{nom}, 1}\right) \exp \left[-\left(\Delta \mathrm{F}_{2}^{*}-\Delta \mathrm{F}_{1}^{*}\right) /\left(4 \mathrm{k}_{B} \mathrm{~T}\right)\right]
\end{aligned}
$$

Initially, we tried to fit this data using

$$
\mathrm{F}\left(\mathrm{pH}^{\mathrm{pH}} \mathrm{nom}_{\mathrm{n}}\right)=\mathrm{f}_{\mathrm{nom}}(\mathrm{pH})
$$


In (3.12.2) and $f\left(\mathrm{pH}_{\mathrm{nom}}(\mathrm{pH})\right.$ ) as the Integrand in (3.10.3). (All this is formally equivalent to setting $\mathrm{pH}=\mathrm{pH}_{\text {nom }}$ throughout.) These assumptions failed badly to fit the data; in every case, the calculated ratio of the rate of the salt added run to the rate of the corresponding no salt added run was substantially higher than that experimentally observed.

We then tried using the form

$$
\mathrm{F}\left(\mathrm{pH}_{\mathrm{pH}} \mathrm{pH}_{\mathrm{nom}}\right)=\mathrm{hf}^{\prime}(\mathrm{pH})+(1-\mathrm{h}) \mathrm{f}^{\circ}\left(\mathrm{pH}_{\mathrm{nom}}\right)
$$

where $h$ is a parameter to be fitted to the experimental data. This form allows for the fact that some of the ionized silanols do not have cations bound to them. The best fit was obtained with $h=0.45$. With this value the KUSS deviation between the calculated and empirical relative rate values was only about $0.09 \mathrm{log}$ units.* This is about the same size as the other residual fitting.errors encountered, and this suggests that, at the very least, possible overfitting or simply incorrect fitting in one place did not spoil the fit in another.

* The RHS deviation that was minimized during this fitting process was defined as follows: let $a_{1}, b_{1}$, and $c_{1}$ be the three empirical relative rate values for the three runs in set 1 , and let $A_{1}, B_{1}$ and $C_{1}$ be the corresponding best calculated values. By definition, the relative rate of the no salt experiment is set equal to unity, so that $\dot{a}_{1}=A_{1}=1$.

The RMS deviation is then

$$
\sigma=\left[\sum_{i=1}^{12}\left(E_{i}^{2}+\left[\log \left(B_{i} / b_{1}\right)-E_{i}\right]^{2}+\left[\log \left(C_{1} / c_{1}\right)-E_{i}\right]^{2}\right) / 36\right]^{1 / 2}
$$

where $E_{i}=\left[\log \left(B_{i} / b_{i}\right)+\log \left(C_{i} / c_{i}\right)\right] / 3$. This definition reflects the fact that the "rate" determined for the reference run is no less subject to experimental error than are the "rates" determined for the two salt added runs in the set. 

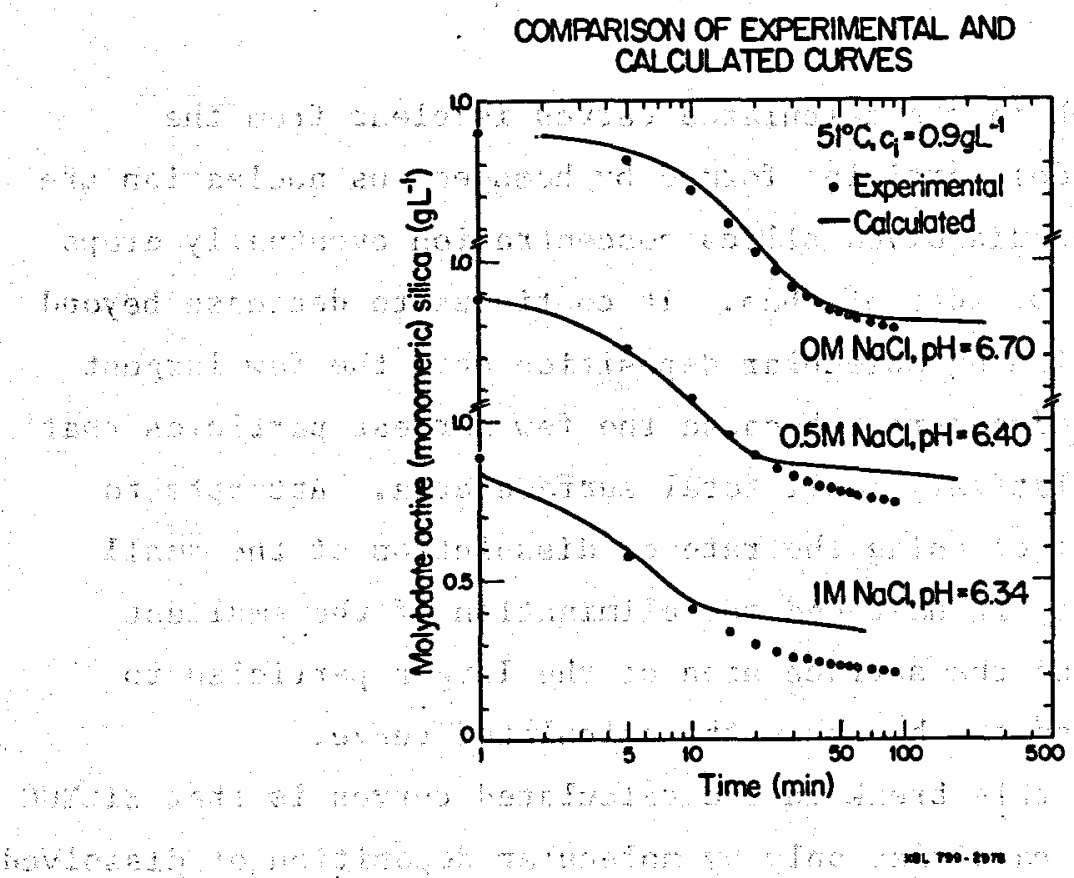

sis.

Fig. 3.20. Solid curves calculated by sILNUC. MAS concentration scale shifted by $0.5 \mathrm{~g} \mathrm{~L}^{-1}$ between curves.

These results have been fully incorporated into the computer program SILNUC. The quality of the agreement between theory and experiment attainable using SILNUC is 11lustrated In Figure 3.20 (as we11 as in Fig. 3.18). In all three cases in Fig. 3.20 , the fit is almost perfect during the early part of the reaction, but there is a deviation toward the end. This deviation increases with increasing salinity.

The immediate cause of the deviation is that the slope of the calculated curve abruptly changes in a way that signifies a marked decrease in the rate of molecular deposition of dissolved silica onto the colloidal silica particles. This break in the curve was also frequently observed in calculations for low salinity (1.e., buffer only) systems, but was usually encountered only after the monomer concentration dropped to values that were not reached in the corresponding experiment because of the very long reaction time that would have been needed to reach them. The monomer concentration at which the break occurred was always higher for calculations commencing at a higher initial concentration and saturation ratio. The increase in the deviation with increasing salinity evident in Figure 3.20 is at least partly mediated by the fact that the saturation ratio increases with salinity. 
The cause of the break in the calculated curves is clear from the SILNUC output: the colloidal particles formed by homogeneous nucleation are numerous and small, and the dissolved silica concentration eventually drops below the solubility level of most of them. It continues to decrease beyond that point because of continuing molecular deposition onto the few largest particles, but at a much reduced rate because the few largest particles that continue to grow have a relatively small total surface area. At tempts to correct this situation by increasing the rate of dissolution of the small particles had 1ittle effect; it hastened the elimination of the smallest particles, but did not cause the surface area of the larger particles to increase enough to eliminate the break in the calculated curve.

The ultimate cause of this break in the calculated curves is that SILNUC models growth of colloidal particles only by molecular deposition of dissolved silica on them. It appears that, in reality, there is also a second particle growth mechanism that becomes relatively important only after the dissolved silica concentration has dropped most of the way from its initial value to the equilibrium solubility. That mechanism can only be growth by collision and adhesion of the particles to form larger particles. The "composite particles" thus formed then grow by molecular deposition of dissolved silica upon them. Essentially, this concretional mechanism provides a way for small particles to be converted into larger ones by a means other than redissolution followed by molecular deposition on other particles.

To be sure, this must occur at earlier stages of the reaction as well, but simply is not very important then because the concentration of MSA is still high enough for most of the particles to continue growing by molecular deposition alone.

That this concretional mechanism of particle growth is ignored in SILNUC is a serious omission but, practically speaking, need not be a fatal one. After all, the appearance of the break is unmistakable, and it is easy to compensate for by judicious extrapolation of that part of the calculated curve that comes before the break. 


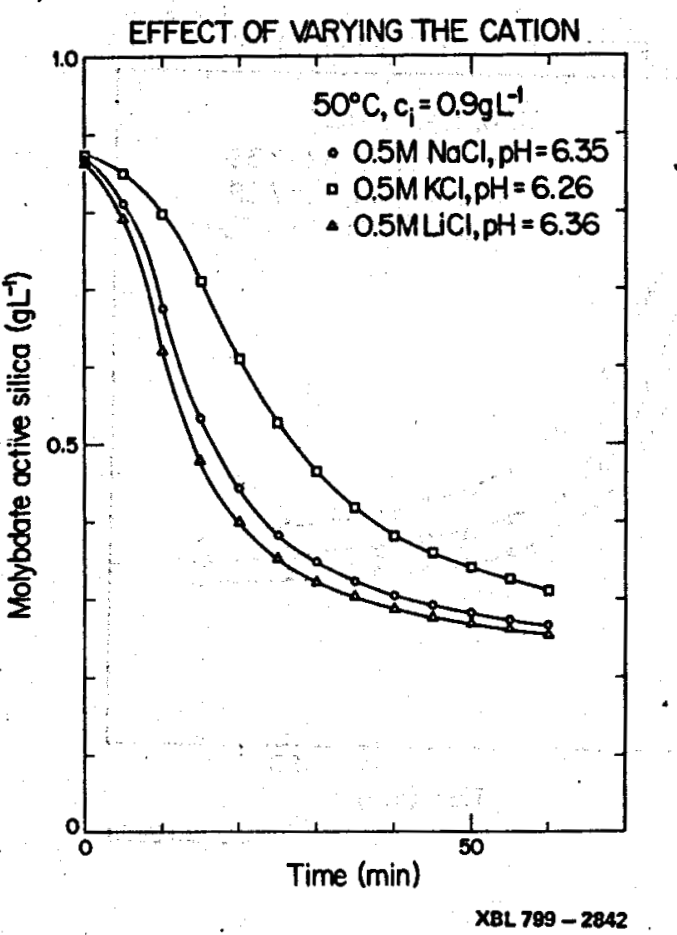

Fig. 3.21.

\section{S3.13 The Effects of 0ther Salts}

Figure 3.21 compares the effects of different alkall metal chlorides on homogeneous nucleation. The sodium and lithium chloride curves appear to be within experimental error of each other, and the deviation of the potassium chloride curve from the other two is exaggerated by 1 ts somewhat higher $\mathrm{pH}$ value. Another set of curves (not shown) which compares the effects of equinormal concentrations of sodium, magnesium and calclum chlorides also. revealed differences between the effects of the three salts that were only about this large.

Figure 3.22 compares the effects of the sodium salts of various anions. It is apparent that both sodium bromide and perchlorate have a greater effect than sodium chloride. Other data showed that this is also true of sodium bicarbonate, lodide, and nitrate.

Figure 3.23 compares the effects of sodium chloride and sodium sulfate, both separately and together. The effect of sodium sulfate is clearly much smaller than that of an equinormal concentration of sodium chloride. (Note that the effect of sodium sulfate is exaggerated by the lower pH of the refer- 


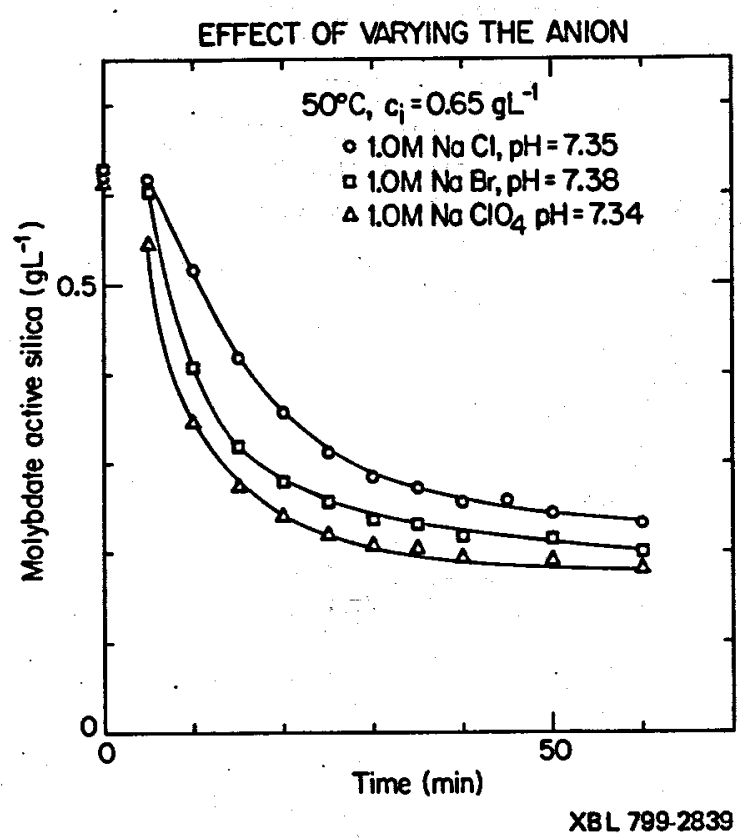

Fig. 3.22.

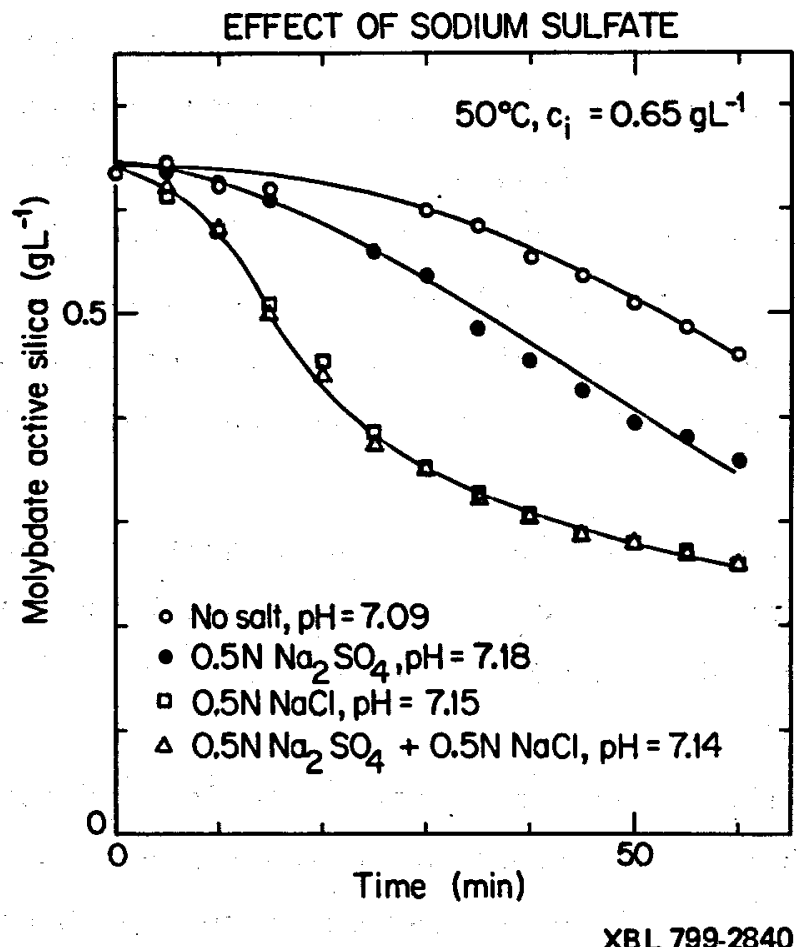

Fig. 3.23 . 
ence curve.) When sodium sulfate is added to a solution that contains sodium chloride, it seems to have no incremental effect at all.

In other experiments it was found that increasing the concentration of maleate in the buffer five-fold had no visible effect, while adding citrate at a $\mathrm{pH}$ above its buffering range actually slowed the reaction down.

To summarize, it was found that (within the range of salts studied) the effect of the added salt varied little with the Identity of the cation. On the other hand, the effect varied markedly with the identity of the anion. All sodium salts of monovalent anions that were tested had marked accelerating effects, with the effect increasing with the size on the anion (i.e., with the partial molal volume of 1 ts sodium salt). The sodium salts of the two divalent anions tested had 1ittle or no effect, and trisodium citrate actually slowed the reaction down.

All of this suggests that the effect of added salts is due to the anions and not the cations; if the opposite were true, one would expect the effect to vary with the Identity of the cation but not with that of the anion. Bauman (1959) performed approximately the same experiments, got approximately the same results, and drew the same conclusion.

Unfortunately, this conclusion conflicts with our interpretation and successful reduction of the low salinity and sodfum chloride added data. It was found that all of this data could be well accounted for by assuming that the rate of molecular deposition and the value of the surface tension at the given temperature and dissolved silica concentration depend only on the density of ionized surface sites on the surface of AS. This, in turn, is determined exclusively by the nature and activities of the cations that are present and the $\mathrm{pH}$. (In this case, by sodium activity and $\mathrm{pH}$.)

The apparent differences between the effects of the sodium salts of the various anions tested are probably due mostly to the differences between the partial molal volumes of the respective salts. Because sodium perchlorate has a larger partial molal volume than sodium chloride, a given molarity of the perchlorate corresponds to a higher molalfty than would the be the case with the same molar concentration of sodium chloride. Asilica concentration of $1 \mathrm{~g} \mathrm{~L}^{-1} \mathrm{In}$ a $1 \mathrm{M} \mathrm{NaClO}_{4}$ solution likewise corresponds to a higher concentration in terms of $\mathrm{g}\left(\mathrm{kg} \mathrm{H}_{2} \mathrm{O}\right)^{-1}$ than would be the case in a $1 \mathrm{M} \mathrm{NaCl}$ solution. Therefore, $1 \mathrm{~g} \mathrm{~L}^{-1}$ in $1 \mathrm{M} \mathrm{NaClO}{ }_{4}$ corresponds to a 
higher saturation ratio than it would in $1 \mathrm{M} \mathrm{NaCl}$ and, all el se being equal, nucleation will be more rapid in the $\mathrm{NaClO}_{4}$ solution. Put more concretely, a mole of a salt that has a larger partial.molal volume will leave less room in a 1iter for molecules of water and MSA to occupy.

All told, the superficially different kinetic effects of the various 1-1 sodium salts are only artifacts that arise from our system of concentration units. If we had worked throughout with concentrations given in terms of moles and grams per kilogram of water, this data would have very probably shown that all 1-1 sodium salts have about the same effect. The same applies to Baumann's (1959) work. The effect of the partial molal volume is even more pronounced in the case of 1-2 and 1-3 salts. The partial molal volumes of these salts are usually smaller than those of 1-1 salts, and the partial volume of a gram equivalent of one of them is smaller still by a factor of two or three. Furthermore, a gram equivalent of a 1-2 or 1-3 salt in a kilogram of water (as opposed to "in a liter of solution") will decrease the solubility of silica less than will a gram equivalent of a 1-1 salt. This is because a gram equivalent of, say, a 1-2 salt contains only $3 / 4$ ths as many ions as a gram equivalent of a 1-1 salt, and because the osmotic coefficient of a 1-2 salt solution is usually smaller than that of a 1-1 solution. Also, a solution that contains a given number of equivalents of a 1-2 or 1-3 salt w111 have a considerably greater lonic strength than one containing the same number of equivalents of 1-1 salt. This means that the sodium ion activity coefficient and activity will be smaller in the 1-2 salt solution even if the sodium ion concentration is the same in both.

Finally, all added salts have a secondary tendency to increase the solubility of AS by enhancing the ionic dissociation of MSA. This is a relatively minor effect that is completely swamped by the solubility decreasing effects in solutions of 1-1 salts, but may be significant in 1-2 or 1-3 salt solutions in which the opposing effects are weaker. This is the most plausible explanation for the reduced rate of polymerization observed in the presence of sodium citrate, because these experiments were performed at high ionic strengths and fairly high $\mathrm{pH}$ values (7.44 and 7.61). It may also be that sodium citrate has a negative partial molal volume or that the citrate ion forms a complex with silica, but we do not know this. 
The above arguments do not explain why the varlous alkali metal and alkaline earth chlorides studied had comparable effects. Ultimately, we can only ascribe that observation to a coincidence that is very fortunate in that 1t simplifies making approximate predictions for a wide range of solution compositions.

\section{S3.14 Methods of Practical Prediction}

Most of our work has been with low salinity (1.e., "buffer only") solutions in which the only cation is sodium, and with solutions that contain a moderate concentration of sodium chloride. This is all the data that has been fitted with kinetic expressions and, strictly speaking, defines the range of conditions in which the results can be directly applied. of course, sodium chloride is the salt that is present in greatest concentration in most geothermal brines, and, as discussed in S3.13, the kinetic effects of other salts are not much different from those of sodium chioride.

The results and discussion in $\$ 3.13$ suggest how to go about defining an "effective sodium chloride concentration" that will be chemically equivalent, Insofar as silica polymerization goes, to an arbitrary mixture of salts, as long as sodium chloride is the most important among them: determine in unfts of moles per liter at room temperature the sum of the concentrations of chloride and bicarbonate. (The latter is usually, but not always, negligible relative to the chloride, (Call this the "effective sodium chloride concentration," and proceed to predict the chemical behavior of the silica in the solution as though sodium chloride at this concentration were the only major salt present. First, convert all concentrations to units of moles or grams per kilogram of water using Eqn. (A3.3.3). Calculate the activity coefficient of the sodium Ion using Eqn. (A3.3.1) and the date in Table A3.3, and then calculate the sodium ion activity as the product of the activity coefficient and the "effective sodium chloride concentration" in molal units. If better data is not avallable, assume that the practical osmotic coeffictent is equal to 0.92 . If the $\mathrm{pH}$ is greater than about 7 , correct the silica concentration for the effect of dissociation using Eqn. $(A 3.3 .2)$,

It is then a straightforward matter to calculate the rate of molecular deposition in a solution that contains sodium chloride using the various for- 


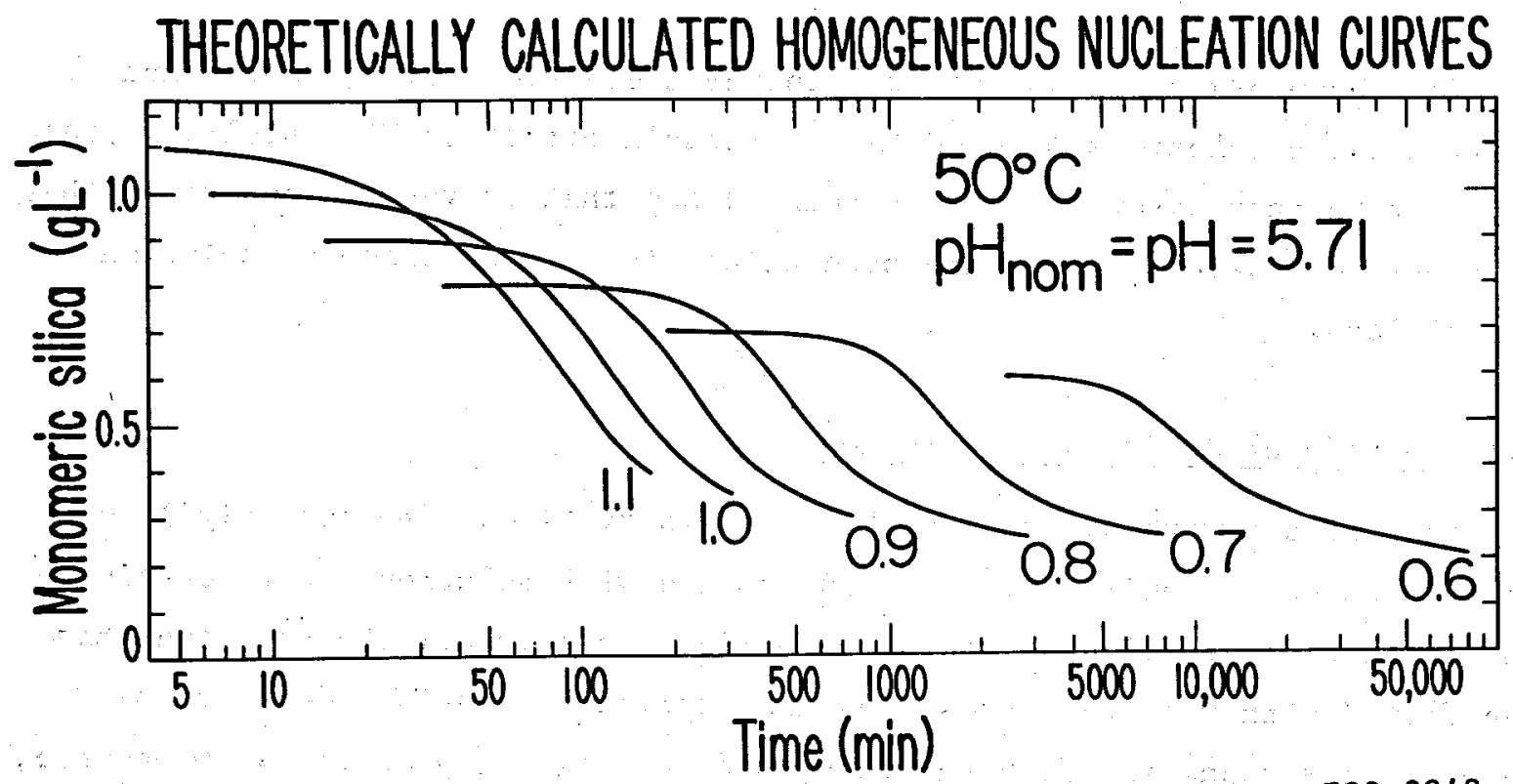

XBL799-2848

Fig. 3.24. Curves in this and the following four Figures calculated using SILNUC. Concentration values are in terms of monomeric silica as in SILNUC. $\mathrm{pH}=\mathrm{pH}_{\text {nom }}=5.71$ was used because $\mathrm{F}(5.71,5.71)=0.100$.

mulas, Figures, and Tables that are given in S3.3, S3.4 and A3.1. The molecular deposition rate in the presence of fluoride is likewlse easy to calculate using the formulas given in $\mathbf{5 3 . 6 .}$

The course of the homogeneous nucleation process in a solution that contains sodium chloride may likewlse be quantitatively predicted using the computer code SILNUC which is listed and documented in Chapter 6 of this report.

Alternatively, the course of homogeneous nucleation at constant temperature and $\mathrm{pH}$ may be estimated using the calculated homogeneous nucleation curves presented in Figures 3.24 to 28 . If the temperature of interest is near to that of one of these Figures, the procedure is as follows:

1. Determine the concentration of dissolved silica and the "effective sodium chloride concentration" in units of grams and moles per kilogram of water, respectively.

2. Determine the true saturation ratio for the dissolved silica under the given conditions, remembering to correctly account for the dissociation of MSA and the effect of the dissolved salt on the solubility (Eqn. 3.11.4). 


\section{THEORETICALLY CALCULATED HOMOGENEOUS NUCLEATION CURVES}

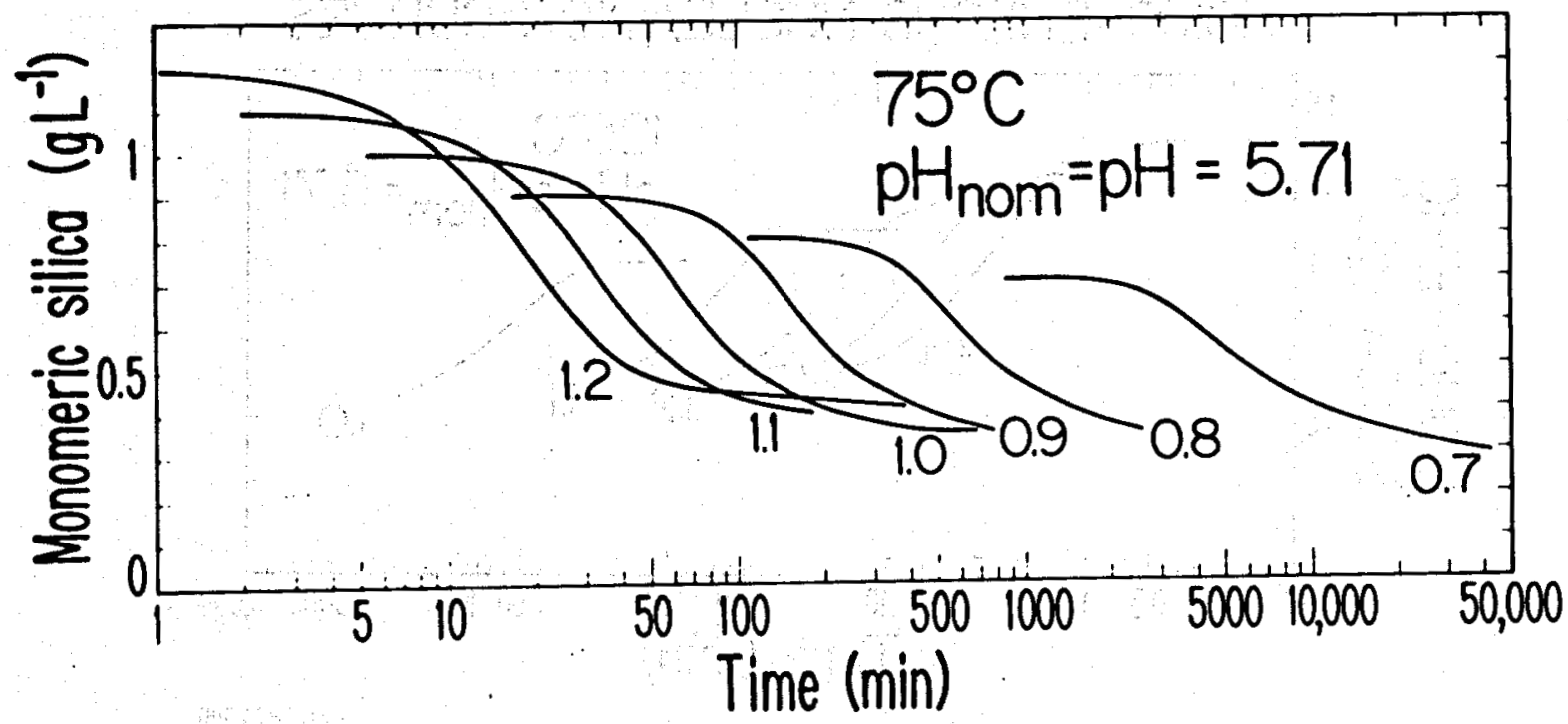

XBL $799-2847$

Fig • 3.25.

THEORETICALLY CALCULATED HOMOGENEOUS NUCLEATION CURVES

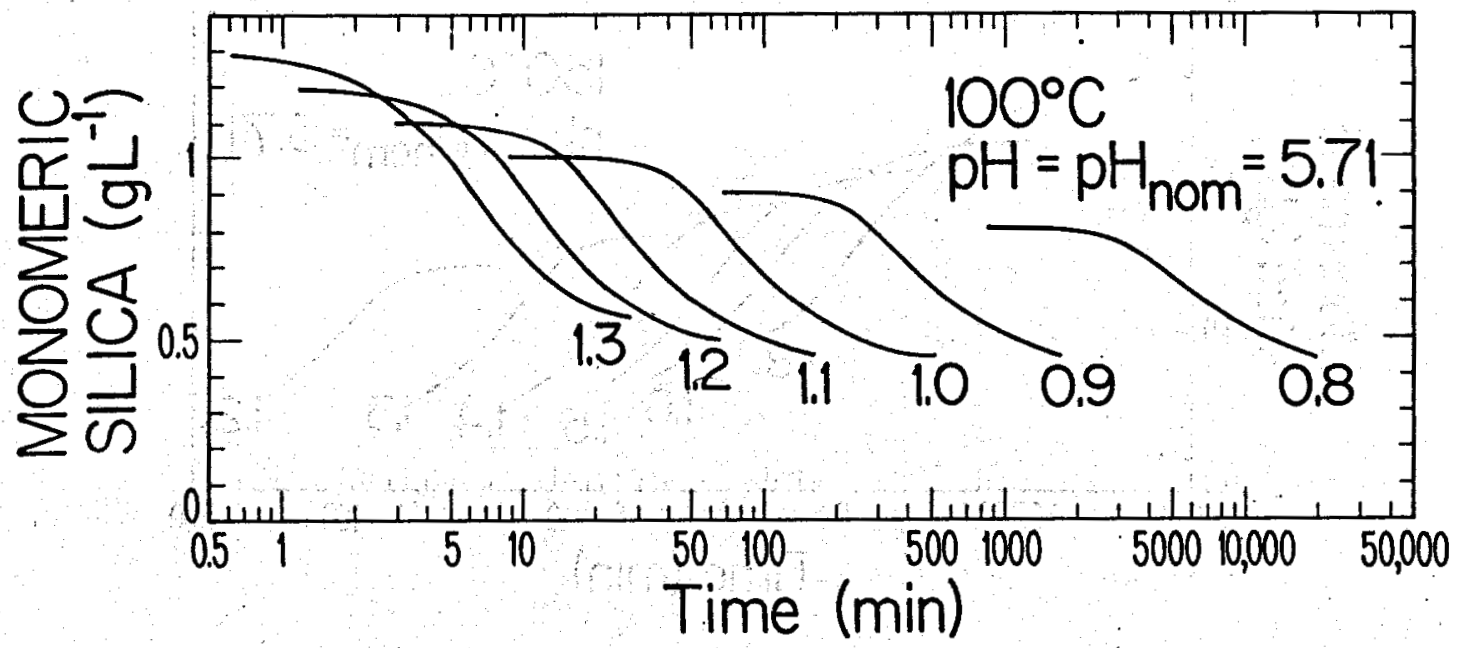

$X B L$ 799-2979

Fig. 3.26 . 


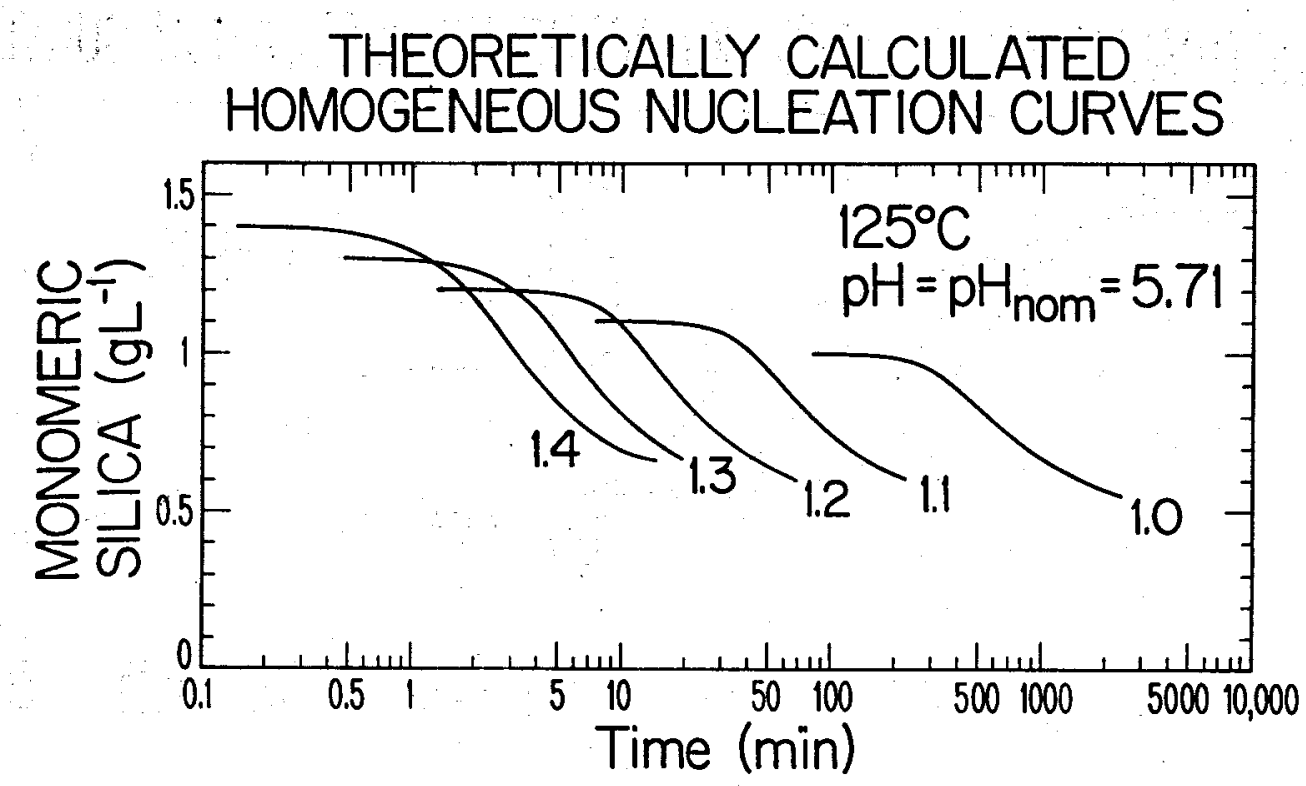

Fig. 3.27.

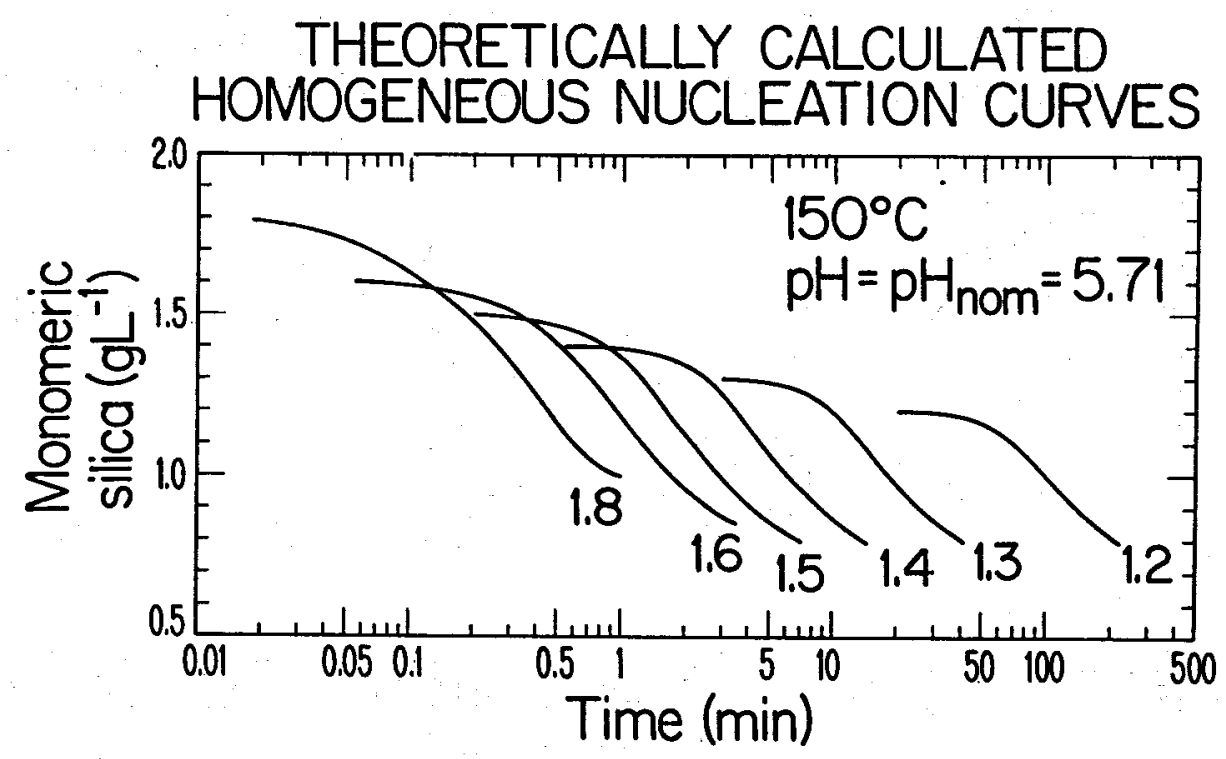

Fif. 3.20 . 
3. Calculate the values of $\mathrm{pH}_{\text {nom }}, F\left(\mathrm{pH}_{,} \mathrm{pH}_{\mathrm{nom}}\right)$ and $\gamma$ for the given conditions using the formulas and Tables in A3.1.

4. Referring to the top part of Table 3.2, find the combination of temperature and concentration for which the value of $S$ is closest to that calculated above. This 1dentifies the calculated "reference curve" which is closest to the conditions of interest. The corresponding value of $S$ found in Table 3.2 w1ll henceforth be be referred to as "the reference value" Sref. Likewise, the "reference values" of the surface tension and absolute temperature may be read from the Table as well as the value of the function Fref which is tabulated in the bottom part of Table 3.2. (Only the reference curve and Fref are actually used.)

If the calculated $S$ value is lower than the tabulated values of $S_{r e f}$ for the given temperature, heterogeneous nucleation is probably dominant and this procedure cannot be used with confidence in any case. Values of $S$ significantly higher than those tabulated are unlikely to be encountered in practice.

The "reference curve" selected In step 4) above is that one among the curves in Figures 3.24 to 28 whose overall shape best matches the course of the homogeneous nucleation process under the given conditions. It remains to calculate the shift along the $\log t$ axis which will give the reference curve its correct time scale. This shift is simply the logarithm of the "Induction time" calculated for the given conditions minus the logarithm of the "induction time" calculated under the conditions that correspond to the reference curve. This difference can be calculated using a simple extension of Eqn. $(3.12 .2):$

$$
\begin{aligned}
\log t= & {\left[\log F(5.71,5.71)-\log F\left(\mathrm{pH}_{\mathrm{pH}} \mathrm{nom}\right)\right] } \\
& +\frac{4 \pi}{3 \times 2.302 \rho_{n}{ }^{2} k_{B}^{3}}\left[(\gamma / T)^{3}(1 \mathrm{n} \mathrm{S})^{-2}-\left(\gamma_{\mathrm{ref}} / \mathrm{T}_{\mathrm{ref}}\right)^{3}\left(\ln \mathrm{S}_{\mathrm{ref}}\right)^{-2}\right]
\end{aligned}
$$

The first term above expresses the effect of the $\mathrm{pH}$ upon the "Induction time" that is mediated by the effect of $\mathrm{pH}$ on the rate of molecular deposition. The second expresses the effects of the surface tension, temperature, 
TABLE 3.2

"Reference Values" to be Used with Figures 3.24 to 28

\begin{tabular}{|c|c|c|c|c|c|}
\hline $\mathbf{T}\left({ }^{\circ} \mathrm{C}\right)$ & 50 & 75 & 100 & 125 & 150 \\
\hline$T(K)=T_{\text {ref }}$ & 323.15 & 348.15 & 373.15 & 398.15 & 423.15 \\
\hline$\gamma_{\text {ref }}\left(\operatorname{erg~} \mathrm{cm}^{-2}\right)$ & 47.47 & 46.22 & 44.96 & 43.71 & 42.46 \\
\hline$C_{1}\left(g^{-1}\right)$ & & & $s_{\text {ref }}$ & $\therefore$ & \\
\hline $\begin{array}{l}0.6 \\
0.7 \\
0.8 \\
0.9\end{array}$ & $\begin{array}{l}3.31 \\
3.86 \\
4.42 \\
4.97\end{array}$ & $\begin{array}{l}2.66 \\
3.04 \\
3.42\end{array}$ & $\begin{array}{l}2.20 \\
2.47\end{array}$ & & \\
\hline $\begin{array}{l}1.0 \\
1.1 \\
1.2 \\
1.3\end{array}$ & $\begin{array}{l}5.52 \\
6.07\end{array}$ & $\begin{array}{l}3.80 \\
4.18 \\
4.56\end{array}$ & $\begin{array}{l}2.75 \\
3.02 \\
3.30 \\
3.57\end{array}$ & $\begin{array}{l}2.07 \\
2.28 \\
2.48 \\
2.69\end{array}$ & $\begin{array}{l}1.94 \\
2.10\end{array}$ \\
\hline $\begin{array}{l}1.4 \\
1.5 \\
1.6 \\
1.8\end{array}$ & & & & 2.90 & $\begin{array}{l}2.26 \\
2.42 \\
2.58 \\
2.90\end{array}$ \\
\hline
\end{tabular}

Fref

0.6

0.7

0.8

0.9

1.0

1.1

1.2

1.3

\subsection{2}

2.45

2.03

3.45

1.74

2.67

$2.18 \quad 3.02$

1.4

1.53

1.85

1.38

1.61
1.44

2.41

2.02

3.53

1.73

2.75

2.26

3.25

1.52

1.91

2.59

$1.65 \quad 2.15$

1.83

1.59

1.6

1.26 
and saturation ratio that are medlated by their effects on the free energy of formation of the critical nucleus.

For practical use, Eqn. (3.14.1) may be simplified by making appropriate substitutions to give

$$
\log t=-1-\log \mathrm{F}\left(\mathrm{pH}, \mathrm{pH}_{\mathrm{nom}}\right)+1412(\gamma / \mathrm{T})^{3}(\operatorname{ln~S})^{-2}-\mathrm{F}_{\text {ref }}(3.14 .2)
$$

The remaining steps in the calculation are:

5. Evaluate $\Delta \log t$ from (3.14.2) using the values of $r, T$, and $S$ that were determined in steps 2) and 3 ) above, and the value of Fref determined in step 4 ).

6. Shift the "reference curve" chosen in step 4) by $\log t$ along the $\log t$ axis in the corresponding Figure either graphically or mentally. The shift is in the following sense: if the value of $\log t$ is positive, shift the reference curve to the right (that is, "slow it down"); If $\log t$ is negative, shift the curve to the left (that is, "speed it up".)

If the desired temperature is not close to that of any one of the Figures, run through the above procedure twice, bracketing the actual temperature with the two nearest tabulated temperatures. (For example, if the given temperature is $90^{\circ} \mathrm{C}$, go through the procedure, once for $75^{\circ} \mathrm{C}$, and once for $100^{\circ} \mathrm{C}$, with concentration, sailnity and $\mathrm{pH}$ as stated and the same in both cases.

7. Then approximate the sought after result by sketching a curve that is intermediate between those obtained from the two calculations.

A sample calculation. This sample calculation approximately describes brine at Cerro Prieto that has been rapidly flashed down to $100^{\circ} \mathrm{C}$ in one step. The major salts in Cerro Prleto brine are $\mathrm{NaCl}$ and $\mathrm{KCl}$ in a mole ratio of about 10:1. In flashed brine, the total concentration of $\mathrm{Cl}^{-}$is about 0.3 moles $\mathrm{kg}^{-1}$, and we can set the "effective $\mathrm{NaCl}$ concentration" equal to this. The dissolved silica concentration and $\mathrm{pH}$ immediately after flashing are typically about $1.0 \mathrm{~g} \mathrm{~kg}$ and 7.2 , respectively. The fluoride catalyzed pathway may be ignored at this $\mathrm{pH}$. We wish to estimate $\mathrm{R}_{\mathrm{md}}$ under the Initial conditions, and to approximately determine the course of silica polymerization by homogeneous nucleation. 


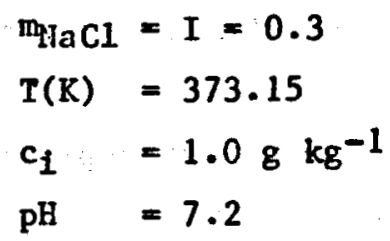

From Eqns. (A3.3.1) and (A3.3.2) and Table A3.3:

$\gamma_{\mathrm{Na}}+=0.685$

$\gamma_{\text {sil }}=0.650$

$\alpha_{s 11}=0.019$

From Eqns. (3.11.1) and (3.11.4):

$c_{0}(0)=0.364$

$c_{\mathrm{o}}\left(\mathrm{m}_{\mathrm{HaCl}}\right)=0.360$

Then

$\left[\mathrm{Na}^{+}\right]=0.3 \times 0.685=0.205$

$c_{i}\left(1-\alpha_{s i 1}\right)=0.981$

$s_{a}=c_{i}\left(1-\alpha_{s 11}\right) / c_{0}(0)=0.981 / 0.364=2.695$

$S=0.981 / 0.360=2.725$

Frow Eqn. (3.3.7)

$\mathrm{pH}_{\text {nom }}=7.20+\log (0.205 / 0.069)=7.67$

From Tables $A 3.1$ and 2 and Eqns. (A3.1.2), (A3.1.3), and (A3.1.5)

$F(7.20,7.67)=0.45 \times 1.284+0.55 \times 2.070=1.716$

$I(7.20,7.67)=0.45 \times 0.0970+0.55 \times 0.1912=0.1488$

$\gamma=63.68-(0.049+0.2174 \times 0.1488) \times 373.15=33.32$

From Table 3.2, the reference curve is the one for $c_{1}=1.0$ and $100^{\circ} \mathrm{C}$ in Fig 3.26 , and

$F_{\text {ref }}=2.41$

From Fig . 3.10, we determine that, at $100^{\circ} \mathrm{C}, c_{1}\left(1-\alpha_{s 11}\right)=0.981$, and $\mathrm{pH}=\mathrm{pH}_{\mathrm{nom}}=7.0, \mathrm{R}_{\mathrm{nd}}=2.2 \mathrm{E}-7 \mathrm{~g} \mathrm{~cm}^{-2} \mathrm{~min}^{-1}=0.53 \mathrm{~mm} \mathrm{yr}^{-1}$. In this case the rate correction factor $(1-1 / 5) /\left(1-1 / \mathrm{S}_{a}\right)$ is obviously so close to unity that we need not bother with it. Nultiplying the value read off of $\mathrm{Fig} .3 .10$ by the value of $\mathrm{F}\left(\mathrm{pH}, \mathrm{pH}_{\mathrm{nom}}\right)$, we obtain the desired result:

$R_{\mathrm{md}}=2.2 \mathrm{E}-7 \mathrm{~g} \mathrm{~cm} \mathrm{~cm}^{-2} \mathrm{~min}^{-1} \times 1.716=3.8 \mathrm{E}-7 \mathrm{~g} \mathrm{~cm}^{-2} \mathrm{~min}^{-1}=0.9 \mathrm{~mm} \mathrm{yr}-1$ From Eqn. (3.14.2):

$$
\log t=-1-0.24+1.00-2.41=-2.64
$$

Therefore, the reference curve in Fig. 3.26 is to be "speeded up" by multiplying 1 ts time scale by $\operatorname{antilog}(-2.64)=2.29 \mathrm{E}-3$. 'In other words, 
under the actual conditions of interest, the reaction runs faster by a factor of about $436=1 / 2.29 \mathrm{E}-3$ than 1s depicted in Fig. 3.26. Examining the reference curve, we see that under the reference conditions, the concentrations would have dropped to $0.8 \mathrm{~g} \mathrm{~kg}^{-1}$ after about 70 minutes, "Speeding this up" by a factor of 436 makes it reach $0.8 \mathrm{~g} \mathrm{~kg}^{-1}$ after about 0.16 minutes.

We conclude that, for most practical purposes, the conversion of dissolved to colloidal silica is almost instantaneous under these conditions.

These results (as well as those generated by SILNUC) must be used carefully and with full awareness of their limitations. First of all, $R_{m d}$ as calculated above is not the rate of scale deposition on a flat surface. Under the conditions of this problem, by far the major mechanism of scale deposition involves electrostatic adhesion of colloidal silicá to surfaces followed by cementation by molecular deposition of dissolved silica between the particles. This process results in scale deposition rates much, much greater than the value of $R_{\text {md }}$ calculated above (see Weres et $81 ., 1980$ ).

Comparing this "shifted curve" estimate of the course of the homogeneous nucleation process with the results obtained by running the same problem with sIlNUC is highly instructive. The "shifted curve" and the curve calculated using SILNUC cross at about $t=0.165 \mathrm{~min}$ and $c=0.77 \mathrm{~g} \mathrm{~kg}^{-1}$. However, the SILNUC curve Is much "flatter", and the two curves cross at a considerable angle. Toward the end of the calculation the difference is large. For example, the siluvic curve shows $c^{\prime}=0.63$ at $t=1 \mathrm{~min}$ and $c=$ 0.55 at $t=5$, while the shifted curve terminates at about $c=0.45$ at $t=1$. Th1s "flatness" of the SILNUC curve is demonstrably due to sILNUC's known inability to properly model the last part of the reaction in a high initial $S$, high salinity medium (see S3.12, S6.7, and Fig. 3.20).

Detailed experimental verification is not possible under such rapid reaction conditions, but avallable synthetic brine data (op. cit., Fig. 2) suggests that the shifted curve is probably more accurate than the sILNUC curve: when the second experimental data point is taken at $t=5$, c $=0.45$.

The results obtained here can also be compared with those of the SILNUC sample problem presented in detail in $\mathrm{S6.8}$. That sample problem involves flashing essentially the same brine down from 300 to $100^{\circ} \mathrm{C}$ slowly enough for the polymerization to begin at about $125^{\circ} \mathrm{C}$. The results obtained 
applying SILNUC to that case are again rather different from the ones obtained here, but not because SILNUC falls again. It does not; rather, the fact of slower cooling and reaction Initiation at a higher temperature change the actual course of the reaction in a way that makes using a "shifted curve" technique inappropriate. These same physical differences actually keep SILNUC "out of trouble" in this case.

This shows how the simple "shifted curve" estimation technique can serve as a check on the results generated by SILNUC and vice-versa. Most of all, it shows that an unthinking "cookbook" approach is inadequate when using SILNUC or the other methods and results presented here. One must develop a basic understanding of the chemical phenomena involved, and be prepared to critically evaluate the results obtained.

\section{S3.15 Inhibition by Aluminum and Boron}

Iler (1973) reported that relatively small amounts of aluminum adsorbed on the surface of colloidal silica drastically decrease both the solubility and the rate of dissolution of the surface layer. The decrease in silica solubility caused by aluminum doping is to be expected in light of the many extremely insoluble aluminosilicate phases known in nature (e.g., clays). The decrease in dissolution rate is in part the kinetic expression of. the reduced solubility, but may also reflect a specific kinetic inhibiting effect. If there is such a specific kinetic inhibiting effect, aluminum doping should also inhibit molecular deposition to some extent.

Figure 3.29 shows the effects of aluminum on the polymerization of silica. In the case of the homogeneous nucleation experiment with aluminum added (solid squares), the aluminum was put into the sodium metasilicate stock solution in the form of sodium aluminate. Apparently, a small amount of an amorphous aluminosilicate phase formed (enough to cause a drop of $0.1 \mathrm{~g}$ $\mathrm{L}^{-1}$ in MAS concentration), prior to or immediately upon mixing, but then further deposition of dissolved silica on these particles continued rather slowly. The colloid-added experiments in Figure 3.29 show the effect of surface doping with aluminum on the rate of molecular deposition onto Ludox particles. The Ludox particles were partially covered with aluminum using the method described by Iler (1973a), and under R. K. Iler's personal supervision in our laboratory. The method consists of first completely deionizing 


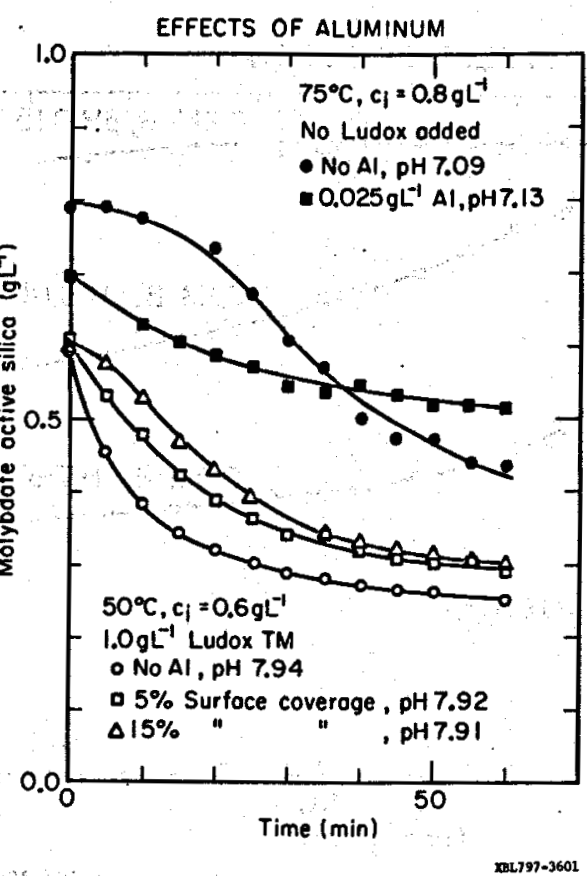

Fig. 3.29. Solid squares: $\mathrm{Na}\left[\mathrm{Al}(\mathrm{OH})_{4}\right]$ added to silica stock solution prior to mlxing with acid and buffer. Open squares and triangles: surface of Ludox doped with aluminum by treating it with aluminum citrate as discussed in text.

a suspension of Ludox, adjusting the $\mathrm{pH}$ to about 7 with dilute $\mathrm{NaOH}$, and then adding the correct amount of aluminum in the form of aluminum citrate with rapid stirring. The "percent surface coverage" is estimated on the assumption that eight atoms of aluminum per square nanometer would be equal to 100 percent surface coverage.

In the case of the aluminum doped Ludox the Inhibiting effect of the aluminum is clearly evident. The shape of the 15 percent surface coverage curve suggests that initially the Inhibiting effect is strong, but that it disappears after the aluminum on the surface of the particles is "buried" by s1lica deposited over 1t.

In many minerals (for example, feldspars) aluminum occurs in tetrahedrally coordinated sites that are chemically equivalent to those in which silicon atoms occur. However, because the atomic number of aluminum is one less than that of silicon, a tetrahedrally coordinated aluminum atom corresponds to an excess negative charge in the aluminosilicate structural framework. In minerals, this charge excess in the framework is balanced by the presence of a cation (sodium, potassium, or calcium in the feldspar minerals) in a framework cavity. 


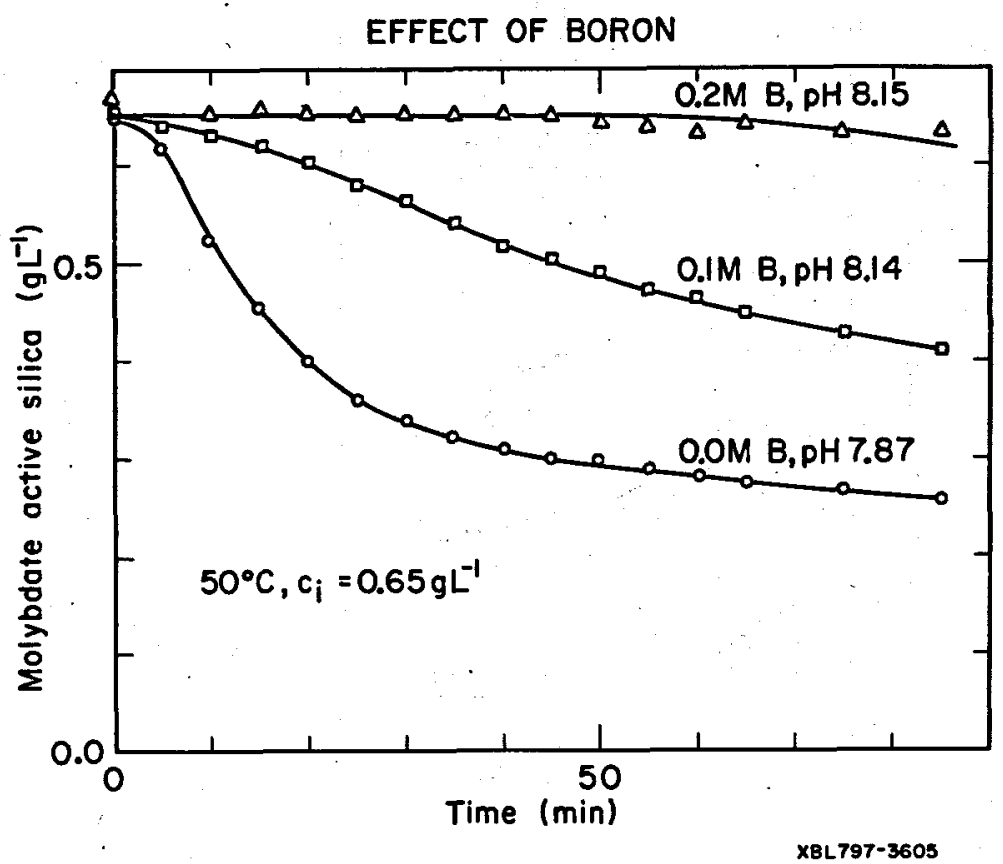

Fig. 3.30: $\mathrm{Na}\left[\mathrm{B}(\mathrm{OH})_{4}\right]$ added to sodium metasilicate stock solution prior to mixing with acid and buffer.

At $\mathrm{pH}$ values above seven, aluminum atoms on the silica surface are probably also tetrahedrally coordinated and, thereby, represent bound negative charges. These negatively charged sites are not and do not resemble lonized stlanol groups. Therefore, they are not reaction loci for the deposition of dissolved silica on the surface. Furthermore, the negative electrical surface potential they produce inhibits the ionization of surface silanols, and this is probably why aluminum doping of the silica surface inhibits the deposition of dissolved silica upon it.

This explanation suggests that the inhibiting effect of surface doping with aluminum should increase with increasing $\mathrm{pH}$, and other data that are not shown here seem to bear this out.

It also suggests that elements that resemble aluminum chemically should also Inhibit silica polymerization. Figure 3.30 shows the effect of adding boron in the form of sodium orthoborate to the solutions in homogeneous nucleation experiments. The sodium orthoborate was added to the sodium metasilicate stock solution. The inhibiting effect is obvious. The fact that the no added boron curve is at a lower $\mathrm{pH}$ makes no difference because this is just in the range of weak dependence of rate on $\mathrm{pH}$ (see s3.3). 
Some metal lons are also known to be very strong inhibitors of the fluoride catalyzed reaction pathway. This was first demonstrated by Iler (1952) for aluminum, beryllium and thorium, and is due to the powerful complexing of fiuoride by the lons of these elements. We found that adding $0.03 \mathrm{~g} \mathrm{~L}^{-1}$ Al to a solution that contained $0.02 \mathrm{~g} \mathrm{~L}^{-1} \mathrm{~F}$ and $1.3 \mathrm{~g} \mathrm{~L}^{-1}$ $\mathrm{SiO}_{2}$ at $\mathrm{pH} 2.48$ and $50^{\circ} \mathrm{C}$ completely blocked the fluoride catelyzed reaction pathway and caused the reaction to proceed exactly as though no fluoride were present. Adding fluoride alone under these conditions had the usual catalytic effect, and aluminum alone had no effect. This is a good point to remember should one wish to completely inhibit silica polymerization by lowering pH; adding a small amount of aluminum along with the acid w111 cause the fluoride catalyzed pathway to be completely blocked as well.

\section{APPENDICES TO CHAPTER THREE}

\section{A3.1 Empirically Fitted Formulas and Tables for the pH Functions .}

To calculate the value of the function $f(p H)$ or $\left(\right.$ or $\left.f\left(\mathrm{pH}_{\text {nom }}\right)\right)$ proceed as follows.

At 1 ow $\mathrm{pH}, \log \mathrm{f}$ is approximately 1 inear in $\mathrm{pH}$, and for $\mathrm{pH}<5.97$, an approximate one step successive substitution calculation is adequate:

$$
\begin{aligned}
& x=p H-7.6 \\
& f_{o}=\operatorname{ant1log} x=10^{x} \\
& \log f=f_{o} /\left(1 .+6.2 f_{o}\right)
\end{aligned}
$$

The following arbitrary, empirically fitted closed form expression may be used when $5.97<\mathrm{pH}<8.72915$ :

$$
\log f=x-d \log (1+10(x / d))-x /\left(a+b x+c x^{2}\right)(A 3 \cdot 1 \cdot 1 c)
$$

where

$$
\begin{aligned}
& a=9.6538 \\
& b=1.7901 \\
& c=4.1811 \\
& d=2.113
\end{aligned}
$$

When $\mathrm{pH}>8.72915$ (which corresponds to $\mathrm{x}>\mathrm{B} / 2=1.12915$ ) use the following symmetry relationship: 
$f(x)=1-f(B-x)$

where $B^{\prime}=B^{\prime} / 2.302=2.2583$

and either ( $A 3.1 .1 \mathrm{~b}$ or $\mathrm{c}$ ) as appropriate to evaluate $\mathrm{f}(\mathrm{B}-\mathrm{x}$ ).

And then

$$
\begin{aligned}
& \mathrm{f}^{\prime}(\mathrm{pH})=\mathrm{f}(\mathrm{pH}) / 0.118913 \\
& \mathrm{~F}\left(\mathrm{pH}, \mathrm{pH}_{\text {nom }}\right)=0.45 \mathrm{f}^{\prime}(\mathrm{pH})+0.55 \mathrm{f}^{\prime}\left(\mathrm{pH}_{\mathrm{nom}}\right)
\end{aligned}
$$

The values of $f^{\prime}(\mathrm{pH})$ are presented In tabular form in Table A3.1 and in graphical form in Fig. 3.4 .

The value of the integral function defined by Eqn. (3.10.3) may be evaluatéd as

$$
I\left(\mathrm{pH}, \mathrm{pH}_{\mathrm{nom}}\right)=0.451(\mathrm{pH})+0.55 \mathrm{i}\left(\mathrm{pH}_{\mathrm{nom}}\right) .
$$

where

$$
i(\mathrm{pH})=\int_{-\infty}^{\mathrm{pH}} f\left(\mathrm{pH}^{\prime}\right) \mathrm{dpH^{ \prime }}
$$

The function $i(\mathrm{pH})$ was first calculated by numerically integrating values of $f(\mathrm{pH})$ given by Eqns. (A3.1.1). These values were then fitted using the arbitrary closed form, analytic expressions given below.

For $\mathrm{pH}<5.97$, use the formula

$$
i=\operatorname{antilog}(1+6.2 \text { antilog } x) / 6.2
$$

with $x$ the same as before.

For $5.97<\mathrm{pH}<8.72915$ use

$$
1=\operatorname{antilog}\left(-0.75924+0.58993 x-0.11292 x^{2}\right)
$$

For $\mathrm{pH}>8.72915$ use the symmetry relation

$$
I(x)=I(B-x)+x-B / 2
$$

along with one of (A3.1.4a or b) as appropriate.

The values of $i(\mathrm{pH})$ are presented in tabular form in Table A3.2.

The algorithms above are contained in FORTRAN coded form in subroutine PHF in SILNUC. This subroutine was used to generate the values in Tables A3.1 and $\mathrm{A} 3.2$.

Finally,

$$
\gamma=63.68-\left[0.049+0.2174 \mathrm{I}\left(\mathrm{pH}, \mathrm{pH}_{\mathrm{nom}}\right)\right] \mathrm{T}
$$


Table A3.1

Values of $\mathrm{f}^{\prime}(\mathrm{pH})$ vs. $\mathrm{pH}$

\begin{tabular}{|c|c|c|c|c|c|c|c|c|c|c|}
\hline $\begin{array}{l}00 \\
10 \\
20 \\
30 \\
40 \\
50 \\
60 \\
70\end{array}$ & $\begin{array}{l}.00211 \\
.00265 \\
.00334 \\
.00420 \\
.00529 \\
.00065 \\
00836 \\
.01050 \\
01320 \\
.01657 \\
.02080 \\
00261 \\
.0327 \\
.0409 \\
.0511 \\
.0637 \\
.0792 \\
.0982 \\
.1214 \\
.1493 \\
.1855 \\
.225 \\
.273 \\
.328 \\
.392 \\
.467 \\
.552 \\
.648 \\
.754 \\
.872 \\
1.000 \\
1.138 \\
1.284 \\
1.439 \\
1.601 \\
1.769 \\
1.944 \\
2.12 \\
2.31 \\
2.50 \\
2.70 \\
2.90 \\
3.10 \\
3.31 \\
3.52 \\
3.72 \\
3.93 \\
4.14 \\
4.36 \\
4.56\end{array}$ & $\begin{array}{l}1.00216 \\
5.00272 \\
4.00342 \\
0.00430 \\
9.00541 \\
5.00680 \\
600855 \\
0.01675 \\
0.01350 \\
0.01696 \\
0.02128 \\
.0267 \\
.0334 \\
.0418 \\
.0522 \\
.0651 \\
.0809 \\
.1003 \\
.1239 \\
.1524 \\
.1892 \\
.230 \\
.278 \\
.334 \\
.399 \\
.475 \\
.561 \\
.658 \\
.766 \\
1.884 \\
1.013 \\
1.152 \\
1.300 \\
1.655 \\
1.718 \\
1.967 \\
2.14 \\
2.33 \\
2.53 \\
2.12 \\
2.92 \\
3.12 \\
3.33 \\
3.54 \\
3.75 \\
3.95 \\
4.16 \\
4.38 \\
4.58 \\
.52\end{array}$ & $\begin{array}{l}6.00221 \\
2.00278 \\
2.00350 \\
0.00440 \\
1.00553 \\
0.00696 \\
5.00975 \\
5.01100 \\
0.01381 \\
6.01735 \\
.02176 \\
.0273 \\
.0342 \\
.0427 \\
.0534 \\
.0665 \\
.0827 \\
.1025 \\
.1265 \\
.1555 \\
.1929 \\
.234 \\
.283 \\
.340 \\
.407 \\
.483 \\
.570 \\
.668 \\
.777 \\
6897 \\
1.027 \\
1.166 \\
1.315 \\
1.471 \\
1.634 \\
1.804 \\
1.980 \\
2.16 \\
2.351 \\
2.54 \\
2.74 \\
2.94 \\
3.14 \\
3.35 \\
3.56 \\
3.77 \\
3.97 \\
4.18 \\
4.40 \\
4.60\end{array}$ & $\begin{array}{l}1.00226 \\
8.00284 \\
0.00358 \\
0.00450 \\
3.00566 \\
6.00712 \\
5.00895 \\
0.01125 \\
1.01413 \\
5.01774 \\
6.02226 \\
.0279 \\
.0349 \\
.0437 \\
.0546 \\
.0680 \\
.0545 \\
.1047 \\
.1292 \\
.1587 \\
.1967 \\
.239 \\
.288 \\
.346 \\
.414 \\
.491 \\
6579 \\
.678 \\
6788 \\
6009 \\
1.040 \\
1.181 \\
1.330 \\
1.487 \\
1.651 \\
1.621 \\
1.997 \\
2.18 \\
2.37 \\
2.56 \\
2.76 \\
2.96 \\
3.16 \\
3.37 \\
3.58 \\
3.79 \\
4.00 \\
4.21 \\
4.42 \\
4.63\end{array}$ & $\begin{array}{l}6.00231 \\
4.00291 \\
8.00366 \\
0.00461 \\
6.00579 \\
2.00729 \\
5.00916 \\
5.01151 \\
3.01446 \\
.01815 \\
6.02277 \\
.0285 \\
.0357 \\
.0447 \\
.0558 \\
.0695 \\
.0863 \\
.1069 \\
.1319 \\
.1620 \\
.2006 \\
.243 \\
.294 \\
.353 \\
.421 \\
.500 \\
.589 \\
.689 \\
.800 \\
.922 \\
1.054 \\
1.195 \\
1.345 \\
1.503 \\
1.668 \\
1.839 \\
2.015 \\
2.20 \\
2.39 \\
2.58 \\
2.78 \\
2.98 \\
3.18 \\
3.39 \\
3.60 \\
3.81 \\
4.02 \\
4.23 \\
4.44 \\
4.65 \\
.65\end{array}$ & $\begin{array}{l}1.0023 \\
1.002 \\
6.003 \\
1.0047 \\
9.005 \\
9.0074 \\
6.0093 \\
1.0117 \\
6.0147 \\
5.0189 \\
7.023 \\
.0292 \\
.0366 \\
.0457 \\
.0570 \\
.071 \\
.0882 \\
61092 \\
01347 \\
.1653 \\
.2046 \\
.248 \\
.299 \\
.359 \\
.428 \\
.508 \\
.598 \\
.700 \\
.812 \\
.935 \\
1.068 \\
1.210 \\
1.361 \\
1.519 \\
1.684 \\
1.856 \\
2.033 \\
2.22 \\
2.40 \\
2.60 \\
2.60 \\
3.00 \\
3.20 \\
3.41 \\
3.62 \\
3.83 \\
4.04 \\
4.25 \\
4.46 \\
4.67\end{array}$ & 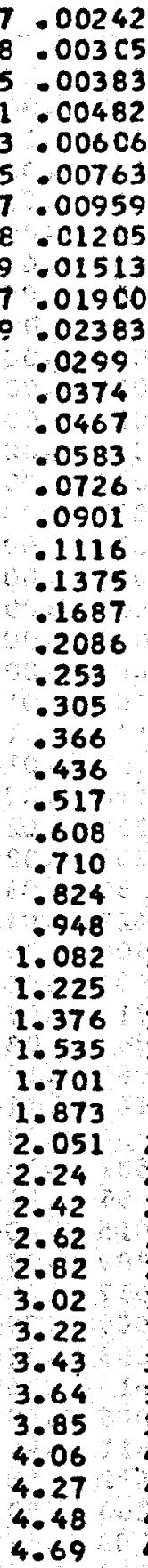 & $\begin{array}{l}2.00248 \\
5.00312 \\
3.00392 \\
2.00493 \\
6.00621 \\
3.00780 \\
.00981 \\
5.01233 \\
3.01548 \\
0.01943 \\
3.02437 \\
.0305 \\
.0382 \\
.0478 \\
.0596 \\
.0742 \\
.0921 \\
.1139 \\
.1404 \\
.1721 \\
.2127 \\
.258 \\
.310 \\
.372 \\
.443 \\
.525 \\
.618 \\
.721 \\
.836 \\
.961 \\
1.095 \\
1.240 \\
1.392 \\
1.552 \\
1.718 \\
.090\end{array}$ & 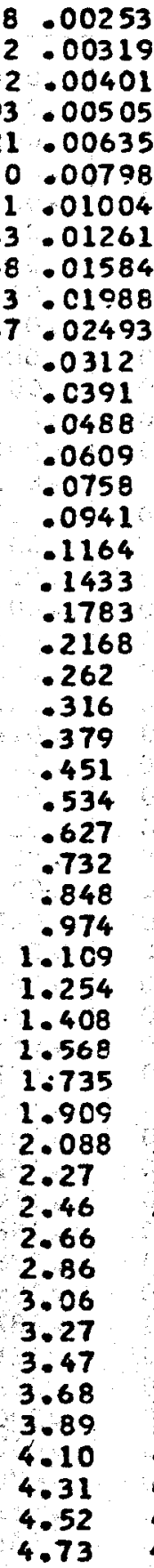 & $\begin{array}{l}.0319 \\
.0400 \\
.0499 \\
.0623 \\
.0775 \\
.0961 \\
1188 \\
11463 \\
1819 \\
.2211 \\
.267 \\
.322 \\
.386 \\
.459 \\
.543 \\
.637 \\
.743 \\
.860 \\
.987 \\
1.124 \\
1.269 \\
1.423 \\
1.585 \\
1.752 \\
1.926 \\
2.106 \\
2.29 \\
2.48 \\
2.68 \\
2.88 \\
3.08 \\
3.29 \\
3.49 \\
3.70 \\
3.91 \\
4.12 \\
4.34 \\
4.54 \\
4.75\end{array}$ \\
\hline
\end{tabular}


Table A3.2

Values of $\mathrm{I}(\mathrm{pH})$ vs. $\mathrm{pH}$

\begin{tabular}{|c|c|c|c|c|c|c|c|c|c|c|}
\hline $\mathrm{pH}$ & 0. & .01 & .02 & .03 & .04 & .05 & .06 & .07 & .08 & .09 \\
\hline $\begin{array}{l}4.00 \\
4.10 \\
4.20 \\
4.30 \\
4.40 \\
4.50 \\
4.60 \\
4.70 \\
4.80 \\
4.90 \\
5.00 \\
5.10 \\
5.20 \\
5.30\end{array}$ & $\begin{array}{l}.0001 \\
.0001 \\
.0002 \\
.0002 \\
.0023 \\
.0003 \\
.0004 \\
.0005 \\
.0007 \\
.0009 \\
.0011 \\
.0014 \\
.0017 \\
.0021 \\
.0027 \\
.0034 \\
.0042 \\
.0053 \\
.0066 \\
.0082 \\
.0102 \\
.0126 \\
.0156 \\
.0192 \\
.0235 \\
.0285 \\
.0345 \\
.0415 \\
.0497 \\
.0592 \\
.0702 \\
.0827 \\
.0970 \\
.1131 \\
.1313 \\
.1516 \\
.1741 \\
.1989 \\
.2261 \\
.2556 \\
.2875 \\
.3217 \\
.3582 \\
.3966 \\
.4370 \\
.4789 \\
.5221 \\
.5663 \\
.6186 \\
.6748\end{array}$ & $\begin{array}{l}.0001 \\
.0001 \\
.0002 \\
.0002 \\
.0003 \\
.0004 \\
.0004 \\
.0006 \\
.0007 \\
.0009 \\
.0011 \\
.0014 \\
.0017 \\
.0022 \\
.0027 \\
.0034 \\
.0043 \\
.0054 \\
.0067 \\
.0083 \\
.0104 \\
.0129 \\
.0159 \\
.0196 \\
.0239 \\
.0291 \\
.0352 \\
.0423 \\
.0506 \\
.0602 \\
.0713 \\
.0841 \\
.0985 \\
.1149 \\
.1332 \\
.1537 \\
.1765 \\
.2015 \\
.2289 \\
.2587 \\
.2908 \\
.3253 \\
.3619 \\
.4006 \\
.4411 \\
.4832 \\
.5265 \\
.5708 \\
.6242 \\
.0805\end{array}$ & 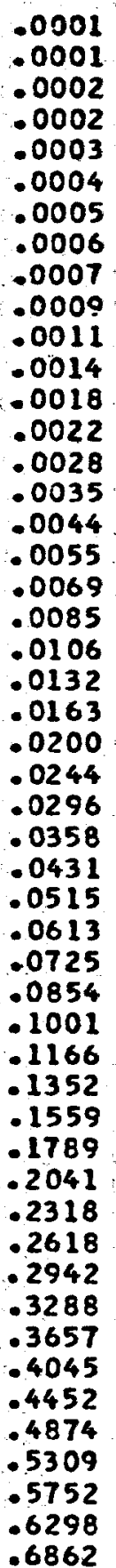 & $\begin{array}{l}.0001 \\
.0001 \\
.0002 \\
.0002 \\
.0003 \\
.0004 \\
.0005 \\
.0006 \\
.0007 \\
.0009 \\
.0012 \\
.0015 \\
.0018 \\
.0023 \\
.0029 \\
.0036 \\
.0045 \\
.0056 \\
.0070 \\
.0087 \\
.0109 \\
.0135 \\
.0166 \\
.0204 \\
.0249 \\
.0302 \\
.0365 \\
.0439 \\
.0524 \\
.0623 \\
.0738 \\
.0868 \\
.1016 \\
.1184 \\
.1372 \\
.1581 \\
.1813 \\
.2068 \\
.2347 \\
.2649 \\
.2975 \\
.3324 \\
.3695 \\
.4085 \\
.4494 \\
.4917 \\
.5353 \\
.5798 \\
.6354 \\
.6919\end{array}$ & $\begin{array}{l}.0001 \\
.0002 \\
.0002 \\
.0002 \\
.0003 \\
.0004 \\
.0005 \\
.0006 \\
.0008 \\
.0009 \\
.0012 \\
.0015 \\
.0019 \\
.0023 \\
.0029 \\
.0037 \\
.0046 \\
.0058 \\
.0072 \\
.0089 \\
.0111 \\
.0138 \\
.0170 \\
.0208 \\
.0254 \\
.0308 \\
.0372 \\
.0447 \\
.0534 \\
.0634 \\
.0750 \\
.0882 \\
.1032 \\
.1202 \\
.1391 \\
.1603 \\
.1837 \\
.2095 \\
.2376 \\
.2681 \\
.3009 \\
.3360 \\
.3733 \\
.4125 \\
.4535 \\
.4960 \\
.5397 \\
.5853 \\
.6410 \\
.6976\end{array}$ & $\begin{array}{l}.0001 \\
.0002 \\
0002 \\
.0002 \\
.0003 \\
.0004 \\
.0005 \\
.0006 \\
.0008 \\
.0010 \\
.0012 \\
.0015 \\
.0019 \\
.0024 \\
.0030 \\
.0038 \\
.0047 \\
.0059 \\
.0073 \\
.0091 \\
.0114 \\
.0141 \\
.0173 \\
.0212 \\
.0259 \\
.0314 \\
.0379 \\
.0455 \\
.0543 \\
.0645 \\
.0762 \\
.0896 \\
1048 \\
.1220 \\
.1412 \\
.1625 \\
.1862 \\
.2122 \\
.2405 \\
.2713 \\
.3043 \\
.3397 \\
.3771 \\
.4166 \\
.4577 \\
.5004 \\
.5441 \\
.5909 \\
.6466 \\
.7033\end{array}$ & 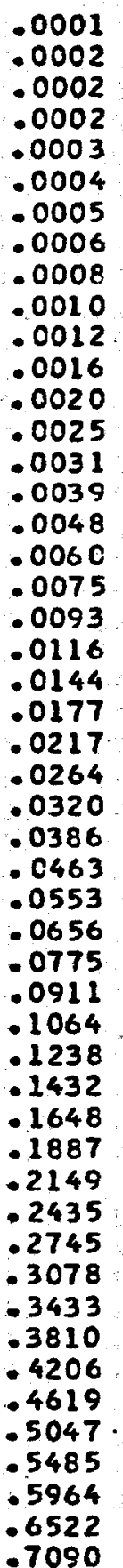 & 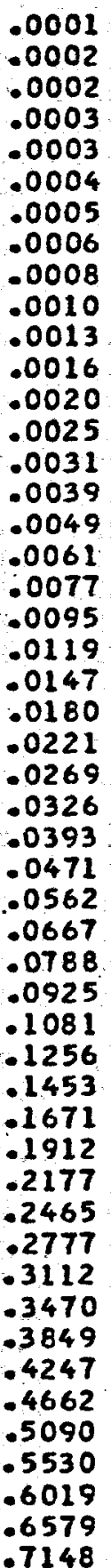 & $\begin{array}{l}.0001 \\
.0002 \\
.0002 \\
.0003 \\
.0003 \\
.0004 \\
.0005 \\
.0007 \\
.0008 \\
.0010 \\
.0013 \\
.0016 \\
.0020 \\
.0026 \\
.0032 \\
.0040 \\
.0050 \\
.0063 \\
.0073 \\
.0057 \\
.0121 \\
.0150 \\
.0184 \\
.0225 \\
.0274 \\
.0332 \\
.0400 \\
.0480 \\
.0572 \\
.0679 \\
.0801 \\
.0940 \\
.1098 \\
.1275 \\
.1473 \\
.1694 \\
.1937 \\
.2204 \\
.2495 \\
.2809 \\
.3147 \\
.3507 \\
.3888 \\
.4288 \\
.4704 \\
.5134 \\
.5574 \\
.6075 \\
.6635 \\
.7205\end{array}$ & 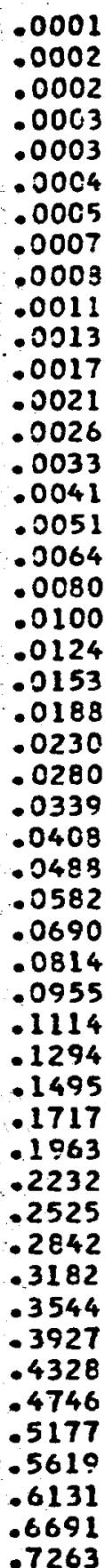 \\
\hline
\end{tabular}




\section{A3.2 Further Experimental Deta11s}

The continuous flow kinetic system. Manual sampling of solutions held In a waterbath at $75^{\circ} \mathrm{C}$ was uncomfortable because of the steam rising from $1 t$, and would have been impossible at $100^{\circ} \mathrm{C}$. Therefore, we devised a system with a closed reaction vessel and provision for continuous automatic sampling for work with boiling solutions. Th1s system is depicted in Fig. 3.1.

The reaction vessel is an electric heater mantled, 1-L, three-necked flask. A continuous stream of the experimental solution is drawn from the flask by a peristaltic pump. This pump also pumps a stream of sulfuric actd solution and a stream of ammonium molybdate reagent solution. The sulfuric acid stream is mixed with the experimental solution stream as soon as possible after the latter leaves the flask, and this immedlately quenches the reaction. The mixture of experimental solution and sulfuric acid is then drawn through the pump, and mixed with the molybdate reagent. The resulting mixture then goes through a two minute delay coll In a waterbath held at about $43^{\circ} \mathrm{C}$ to develop the molybdate yellow color, and finally to the spectrophotometer.

The peristaltic pump used was a Manostat Cassette pump which has separate slow and fast drive shafts and capacity for up to ten pumping cassettes. The spectrophotometer was a (double beam) Perkin Elmer 550 equipped with a flow through ce11. The spectrophotometer was slightly modified so that the sample stream was not regulated by the instrument's controls after it left the cell. (Therefore, the peristaltic pump completely controlled the flow rate through the sample ce11.) The spectrophotometer was operated in the concentration mode, which made 1 possible to adjust the signal amplitude to a conventent range. The spectrophotometer signal was continuously recorded by an ordinary strip chart recorder.

The middle one of the three necks of the flask was fitted with a refluxIng column that was cooled by cold tap water flowing through the facket. This served to minimize water loss by evaporation. Another neck was fitted with a thermometer. The third neck was fitted with a $J$ shaped glass sampling tube with $I_{.} D_{0}=1 \mathrm{~mm}$ and O.D. $_{0} 7 \mathrm{~mm}$. Th1s shape was chosen so that bubbles 
from the bolling reaction solution would not be sucked into the sample stream. A Teflon tube with I.D. $=1 / 32^{\prime \prime}$ was attached to the $\mathrm{J}$ tube by jamming it into the $J$ tube, allowing for quick connection when the reaction was started. This Teflon tube was in turn attached to a mixing manifold that was made by drilling out a small block of plastic. Also attached to this mixing manifold was a soft Tygon tube with I.D. $=1 / 32$ " through which a stream of $1.5 \mathrm{~N} \mathrm{H}_{2} \mathrm{SO}_{4}$ was pumped at $7 \mathrm{ml} \mathrm{hr}^{-1}$. The resulting mixture was drawn through the peristaltic pump in a 1/16" I.D. soft Tygon tube at 25 $\mathrm{ml} \mathrm{hr}^{-1}$. The rate at which the experimental solution was withdrawn from the flask was the difference between these two rates, or about $18 \mathrm{ml} \mathrm{hr}^{-1}$. The transit time from the interior of the flask to the quench point within the mixing manifold was about 25 seconds. The sulfuric acid and mixed streams were pumped by two separate cassettes, both of which were driven by the slow drive shaft.

The mixture of experimental solution and sulfuric acid was mixed with a stream of molybdate reagent in a second mixing manifold. The molybdate reagent was drawn through a soft Tygon tube with I.D. $=3 / 16^{\prime \prime}$ and wall thickness of $1 / 32^{\prime \prime}$ at a rate of about $350 \mathrm{ml} \mathrm{hr}{ }^{-1}$. This stream was pumped by its own cassette, which was driven by the fast drive shaft of the pump. The same molybdate reagent formula was used as in the experiments with. manual sampling. (See further below.)

Tests with standards of known silica concentration established that the apparatus responded linearly to MAS concentrations up to $1.2 \mathrm{~g} \mathrm{~L}^{-1}$.

A total reaction volume of $0.4 \mathrm{~L}$ was used (1.e., twice that used at lower temperatures). The acid-buffer mixture was preheated in the reaction flask to $100^{\circ} \mathrm{C}$ while refluxing. The sodium metasilicate solution was preheated in a plastic jar in a boiling water bath. When the latter reached a temperature of $95^{\circ} \mathrm{C}$, it was poured into the reaction flask to initiate the reaction, and the sampling tube was connected to the pump immediately thereafter. The liquid in the flask was rapldiy stirred with a magnetically driven stirring bar at the time of mixing and for a few seconds thereafter. After mixing, the experimental solution rapidly attained a temperature of $100^{\circ} \mathrm{C}$, and remained steady at that value for the duration of the experiment. 
Whenever colloldal silica was added to the solution, it was added about three minutes after the solutions had been mixed. This delay prevented the loss of data from the early minutes of the reaction which would have otherwise been caused by the finite response time of the system. (It usually took about two minutes to go from no silica to a steady, high concentration.)

A room temperature standard solution of either 0.8 or $1.0 \mathrm{~g} \mathrm{~L}^{-1} \mathrm{SiO}_{2}$ was run through the system while the two components of the experimental solution were being preheated. A heated standard was unnecessary, because the temperature of the solution being sampled would have affected the amplitude of the signal only through its effect on the pumping rate, and, in practice, the mixture of experimental solution and sulfuric acid had had enough time to cool down most of the way to room temperature by the time it reached the peristaltic pump. Preliminary tests confirmed that preheating the standard had very 1ittle effect. Also, not preheating it avolded potentially serfous and unpredictable errors due to concentration by evaporation.

A IImited amount of work was also done with the continuous flow system at lower temperatures in order to compare the data it produced with manually generated data. In this case, the reaction vessel was simply a plastic bottle standing in a thermostatted water bath and the solution preparation procedure was exactly the same as for experiments with manual sampling. We found that the data obtained from the two methods was consistent. .

The major advantages of the continuous flow system are:

1) It allows work at $100 \mathrm{o}^{\mathrm{C}} \mathrm{with}$ excellent temperature control.

2) The experiment can be allowed to run with no further effort on the part of the operator once it has been set up.

3) The data traces produced are continuous and have a time resolution on the order of one minute (as compared to five minutes in the case of most of the manually sampled work).

4) The data traces produced are smooth and free of point scatter. The main disadvantages of the continuous flow system are:

1) Maintaining steady pumping rates is a chronic problem. Nearly half of the experiments started had to be aborted due to signal and/or baseline drift. We concluded that this is mostly due to the gradual change of the characteristics of the pump tubing with time. We learned to be 
careful not to kink the tubing while putting it into the cassette, to "break in" new tubing by leaving it clamped in the cassette overnight and then pumping water through it for $a$ while, and to reduce undesirable further distortion once broken in by unclamping the cassettes when the pump was not in use.

2) Only one experiment can be run at a time. This substantially reduced the rate of data generation, and made it impossible to reduce random variations between runs by running them simultaneously. .

3) The continuous traces had to be manually digitized prior to data analysis.

Adsorbed silica determination. The experiments employed to determine the density of adsorbed or surface active silica were of the column elution type. A small plastic column (about $7 \mathrm{ml}$ volume) packed with powdered quartz glass (1.e., solid vitreous silica) was used:

The quartz glass powder was prepared by grinding broken pleces of quartz glass tubing in a Chatterbox. The powder obtained after two minutes of grinding was sieved through a 125 mesh screen. The coarse material retained on the screen was returned to the grinder or discarded. The powder sieved through was then digested in hot $6 \mathrm{~N} \mathrm{HCl}$ to dissolve the contaminating metal particles that resulted from the grinding. Utra fine particles were removed by repeated decantation in water. The acid washed material was then leached in boiling $6 \mathrm{~N} \mathrm{NaOH}$ for 30 minutes to remove the disturbed surface layer produced by the grinding process, sharp edges, etc. After the powder was washed free of alkali and dried; its surface area was determined by nitrogen adsorption (the B.E.T. method). The quartz powder obtained in this manner had a specific surface area of $0.24 \mathrm{~m}^{2} \mathrm{~g}^{-1}$.

7.5 grams of this powder (with a total surface area of $1.8 \mathrm{~m}^{2}$ ) was packed as a slurry into a plastic column fabricated out of flexiglass tubing that measured $10 \mathrm{~cm} \times 1 \mathrm{~cm}$ I.D. The ends of the column were fitted with single hole rubber glass stoppers and a small amount of quartz glass wool to retaln the powder in the tubing. The column was connected to a peristaltic pump. All tubing connections were of teflon silicone to maintain column temperature. The entire column assembly was immersed in a constant temperature water bath set at $50^{\circ} \mathrm{C}$. Just prior to use the column was washed with 1.ON NaOH followed by deionized water. 
Dissolved silica was "adsorbed" onto the surface of the quartz glass in the column by pumping a buffered solution containing the desired concentration of dissolved silica through the column using the peristaltic pump. Different concentrations ranging from 0.1 to $0.4 \mathrm{~g} \mathrm{~L}^{-1}$ were used in different runs. In each case, the solution was buffered with the usual barbitalmaleate buffer system, and its $\mathrm{pH}$ adjusted to 7.50 with dilute sulfuric acid before use. The pumping rate through the column was $1.5 \mathrm{ml} \mathrm{min}^{-1}$, and the solution was recirculated. To allow adequate time for chemical equilibration the pumping was maintained for periods ranging from 1 to 24 hours (usually between 1 and 5 ). Our data suggests that the results obtained are not effected by changing the equilibration time within this range.

At the end of the adsorption period, the column was removed from the water bath and washed with $0.005 \mathrm{~N} \mathrm{HCl}$. The column was then eluted with $0.1 \mathrm{~N}$ $\mathrm{NaOH}$ to remove the adsorbed silica. The eluting solution was collected in fractions of $10 \mathrm{ml}$ volume, and the amount of silica in each fraction was determined by the molybdate blue method until the silica in each fraction had reached a steady state valuc. This final steady state value was interpreted as corresponding to the dissolution of the solid quartz glass rather than the removal of the adsorbed silica from its surface.

To calculate the amount of adsorbed silica, the final steady state amount of silica (per fraction) was subtracted from the amount in each of the early fractions, and these differences summed. In other words, the initial excess of silica coming off of the column (relative to the final steady state value) at the beginning of the elution was attributed to the removal of the relatively more loosely bound and, thereby, more reactive "adsorbed" silica.

Spectrophotometric determination of silica. There are two standard spectrophotometric methods that are routinely employed for the determination of silica in aqueous solution. Both methods use ammonium molybdate to form a colored silicomolybdate complex in an acid medium, the absorbance of which is then measured with a spectrophotometer. For solutions containing relatively large concentrations of dissolved silica the simpler molybdate yellow method is used. At concentrations below about $0.02 \mathrm{~g} \mathrm{~L}^{-1}$ the much more sensitive molybdate blue method 18 recommended. 
The nolybdate blue method involves further reducing the faintly yellow silicowolybdate complex to a very intensely blue complex, thereby extending the sensitivity of the procedure. Any of a number of reducing agents may be used for this purpose; for example, sodium sulfite, sodium bisulfite, stannous chloride, vitanin $C$, or aminonaphthosulfonic acid. The most comnon interfering substance is Inorgantc phosphate which is of ten present in water and various chemicals and reacts with the molybdate reagent much as silica does. It can be chelated with oxalic acid to give a complex that does not interfere with the nolybdate reaction. Therefore, oxalic acid is routinely added to the nixture when the nolybdate blue method is used.

The molybdate yellow method used was that recommended by $\operatorname{Iler}(1979, \mathrm{p} .97)$. llaterials for molybdate analysis.

- Standard silicon reference solution, 1,000 ppn as silicon, Fisher scientific or equivalent.

Working standard solution, $100 \mathrm{ppm}$ Si: dilute $10 \mathrm{ml}$ of reference standard above with V.I. water to $100 \mathrm{ml}$ and store in plastic container. Anuoniun wolybdate stock solution, $10 \%$ : $100 \mathrm{~g}$ ammonium molybdate ; $4 \mathrm{H}_{2} \mathrm{O}$ and $47 \mathrm{nl}$ concentrated $\mathrm{NH}_{4} \mathrm{OH}\left(28 \% \mathrm{NH}_{3}\right)$, diluted to 1 L with D. I. water. Working acid annonium molybdate solution: dilute $100 \mathrm{ml}$ stock molybdate with 500 w1 D.I. water, add $200 \mathrm{ml} 1.5 \mathrm{~N}$ sulfuric acid and mix.

Oxalic acid solution: dissolve 10 grams in D.I. water to give $100 \mathrm{ml}$. Reducing solution: dissolve $0.5 \mathrm{~g}$ 1-amino-2-naphtho1-4-sulfonic acid and $1 \mathrm{~g}$ sodium sulfite in $50 \mathrm{ml}$ b.I. water; add this to a solution of $30 \mathrm{~g}$ sodium bisulfite in 150 wl water.

Suifuric Acid, $1.5 \mathrm{~N}$ : Dilute $41.7 \mathrm{ml}$ concentrated $\mathrm{H}_{2} \mathrm{SO}_{4}$ with D.I. water to give $1 \mathrm{~L}$.

Procedure: nolybdate yellow method. To $40 \mathrm{ml}$ of molybdate working solution contained in a $50 \mathrm{ml}$ volumetric flask add from a pipet $1 \mathrm{ml}$ of the solution to be analyzed for silica. Dilute to $50 \mathrm{ml}$ with D.I. water and mix tnoroughly (incomplete mixing at this stage is the major source of error in this procedure). Allow 3-1/2 minutes for full color development at room teuperature. Then read the absorbance with a spectrophotometer set at 400 nw. Run a reagent blank and at least one standard each time samples are to be analyzed for silica. The molybdate yellow method has been demonstrated to 
be linear up to an $0 . \mathrm{V}$. of 1.5 . One milliliter of the working standard solution typica1ly yielded an $0 . D$. of about 0.155 .

A double beam spectrometer (Perkin Elmer 550) equipped with a flowthrough cell was used. In most cases, a blank formulated from the working nolybdate solution and D.I. water was present in the reference beam.

The molybdate yellow method was used throughout the kinetic experiments.

Procedure: molybdate blue method. Because of the extreme sensitivity of this method all glassware must be avoided. P1ace $30 \mathrm{ml}$ of the working nolybdate reagent in a $50 \mathrm{ml}$ polypropylene volumetric flash (Nalgene or equivalent) and add to it up to $10 \mathrm{ml}$ of sample containing silica to be analyzed. Allow $3-1 / 2$ minutes as before for ful1 color development. Then add $1.5 \mathrm{ml} 10 \%$ oxalic acid solution and wait for two minutes. Finally, add $2 \mathrm{~m} 1$ reducing agent, dilute to nark; and mix. After 5 minutes read the absorbance with a spectrophotometer set at $650 \mathrm{~nm}$. The complex is stable up to 12 hours and obeys Beer's Law. Blank and standard determinations are performed in a similar manier. A dilute silicon standard containing 25-50 ppm of $S 1$ is used with the nolybdate blue nethod.

The molybdate blue method was used in the adsorbed silica determination experiments.

Preparation of a primary silicon standard solution. A primary silicon standard solution was prepared from ultra pure silicon dioxide (99.999\% purity) by fusing it with sodium carbonate in a platinun crucible. The melt was dissolved and diluted to known volume with deionized water and immediately stored in a plastic container. This primary standard was used to calibrate the Fisher scientific reference silicon standard and the sodium netasilicate stock solutions used in the kinetics work.

Materials:

Silicon (IV) oxide, 99.999\% pure, from Alfa Ventron.

Sodiun carbonate, AR grade, Mallinckrodt.

Procedure: mix exactly $2.11982 \mathrm{~g}$ of ultrapure $\mathrm{SIO}_{2}$ with $8.40 \mathrm{~g}$ of anhydrous sodium carbonate (mole ratio $1: 4$ ) in a platinum crucible. Fuse over a Meker burner for 20 minutes. While st11l very hot, touch the bottom of the crucible to cold water. This solidifies the fused material and causes it to separate from the crucible wall which enables it to be easily removed. 
Invert the crucible over a beaker which contains some D.I. water to transfer the pellet of fused material to the beaker. Rinse out the crucible with hot D.I. water and add the rinse water to the beaker. Transfer the contents of the beaker to a $1 \mathrm{~L}$ volumetric flask with careful rinsing. Add D.I. water to a total volume of $1 \mathrm{~L}$ and mix thoroughly. Store in a plastic bottle.

This standard has a silica concentration of $2.11982 \mathrm{~g} \mathrm{~L}^{-1} \mathrm{SiO}_{2}$ and a $\mathrm{pH}$ of 12.5 .

Characterization of colloidal silica sols. Commercial colloidal silica sols (Ludox TM, BS, and SM) were obtained from the du Pont Company which manufactures them: The samples received were carefully characterized before use. This characterization consisted of determining the total weight concentration of $\mathrm{SIO}_{2} \mathrm{in}$ each, and the specific surface area of each.

The procedure for the gravimetric determination of the silica content of the sols was as follows: a carefully weighed sample of the sol containing one to two grams of $\mathrm{SiO}_{2}$ was placed in a platinum crucible and slowly dried In a $100^{\circ} \mathrm{C}$ convection oven or over a warm hot plate. Slow drying was necessary because rapid drying invariably caused splattering that resulted in loss of sample. When the sample was dry, two to three drops of concentrated sulfuric acid were added and the sample heated over a low flame in a well ventilated fume hood. When fuming ceased the sulfuric acid treatment was repeated once more. The purpose of this treatment was to convert all the salt present In the sample to the sulfate form with the liberation of chlorides and nitrates as $\mathrm{HCl}$ and $\mathrm{HNO}_{3}$. The sample was then cooled and the weight of the sample plus crucible recorded.

One or two drops of concentrated sulfuric acid were again added to the sample, and then HF was added dropwise. Approximately two milliliters of HF was required. The crucible was gently rotated with a tong and warmed over a hot plate to facilitate volatilization of the $\mathrm{SiF}_{4}$ formed. After complete liberation of $\mathrm{SIF}_{4}$ the excess sulfuric acid remaining in the crucible was removed over a hotter flame. The HF treatment was then repeated to ensure complete removal of all the silica. The crucible was then weighed again. The difference in weight before and after the HF treatment was the weight of silica present in the original sample. Sillca values obtained in this manner agreed well with those given in the du Pont, product specification sheets. 
Surface area determination by the Sears Titration Method. The specific surface area of the Ludox colloidal silica was determined by the titration method of Sears (1956).

A quantity of silica supension known to contaln exactly 1.50 grams of colloldal $\mathrm{SiO}_{2}$ was weighed into a beaker. $15 \mathrm{grams}$ of $\mathrm{NaCl}$ and enough water to bring the total volume up to $150 \mathrm{ml}$ were added. The beaker was placed into a water bath held at $25^{\circ} \mathrm{C}$ and 1 ts contents allowed to thermally equilibrate. The $\mathrm{pH}$ was monitored with an Orion $\mathrm{pH}$ meter accurate to \pm 0.001 units and the initial $\mathrm{pH}$ was adjusted to 4.0 with $0.1 \mathrm{~N} \mathrm{HCl}$. The solution was then carefully titrated to a final pH of 9.0 with standardized $0.100 \mathrm{~N} \mathrm{NaOH}$, and the volume of base required was recorded. When the endpoint is approached, some time must be allowed after each addition of base to allow the pH value to stabilize before adding more base. (The pH always drifts back down a bit with time.) The specific surface area in $\mathrm{m}^{2} \mathrm{~g}^{-1}$ was then calculated from the formula: $S=32 \mathrm{~V}-25$ where $V$ is the volume in $m 1 s$ of $0.100 \mathrm{~N}$ base used in the titration.

The values for specific area in $\mathrm{m}^{2} \mathrm{~g}^{-1}$ of $\mathrm{SiO}_{2}$ obtained in this way for the three products were:

$\begin{array}{ll}\text { Ludox TM } & 157 \\ \text { Ludox HS } & 242 \\ \text { Ludox SM } & 359\end{array}$

These values are the averages of two determinations each. We estimate the error in them to be less than $2 \%$. By way of comparison, the duPont product literature (duPont Company, no date) gives typical values of 130 , 240 , and 360 for the three products.

\section{A3.3 Sources of Supplementary Data and Further Detalls of Data Reduction}

The mathematical model In SILNUC and our data reduction procedures required certain supplementary data from various sources:

Except as noted below, all lonic equilibria were calculated the old fashioned way, using $\mathrm{pK}$ values extrapolated to zero lonfc strength and single Ion activity coefficients that were approximately evaluated using a simple form of the extended Debye-Hitcke1 theory. It is true that the dissoclation equilibrium concentration products for monosilicic acid and water have been fitted by Busey and Mesmer $(1977,1976)$ using P1tzer's (1973) much 
superior tneory of electrolytes. However, this theory was not used in our present work or incorporated into SILNUC because it does not provide any way to estinate single ion activities. In principle, one can completely avoid using single ion activities in setting up theoretical models, but taking such a course would probably have made our nodel of silica polynerization unmanagable and impractical. Also, other ionic equilibria that arise in our own work and in the study of the equilibriun chenistry of geothernal brines have not yet Deen described using Pitzer's formalism. Therefore, we chose to use the simpler, old fashioned forualisa even though we know that it is less accurate. As it turned out, the only ionic activity coefficient that plays an iuportant role in our tiodel is that for sodium, and this is probably given fairiy accurately by the extended Debye-Hückel theory in the form used by us. after the Pitzer theory has been extended to allow prediction of single ion activity coefficients, it will probably be desirable to incorporate it into SILNUC. This would allow for nore confident extrapolation to ionic strengths greater than about 1.1 (the naxiluum value encountered in nost of our experiments) and to $\mathrm{pH}$ values above 8. We very much doubt that doing so would require that our data be reanalyzed, because nost of it was generated in precisely the range in which changing the electrolyte solution model assuned would have least effect. Likewise, it would be desirable to replace our theoretical estimate of the effect of salts on the solubility of silica (3.11.4) by empirical values. However, in this case the formula that gives the values of the surface tension (A3.1.5) might need to be revised to waintain internal consistency.

The suurces for the formulas we used to calculate the solubility of AS in pure water and the dissociation constant for HF (Eqns. (3.11.1) and (3.6.5), respectively) are cited in the text.

To calculate the $\mathrm{pK}_{\mathrm{d}}$ values for nonosilicic acid and water we used the formulas biven by Busey and Mesmer (1977) and Busey and Mesmer (1976) with the ionic strength set equal to zero.

The subroutine WATER of SILNUC calculates the density and dielectric constant of pure water and the Debye-Hückel constants. In the temperature range $U-15 U^{\circ} \mathrm{C}$, the empirical formula given by by Eisenberg and Kauzmann $(1969, \mathrm{p} .183)$ is used to calculate the density. In the range $150-250^{\circ} \mathrm{C}$, 
our own fit to the stear table values of Irvine and Hartnett (1976) is used. To calculate the dielectric constant over the range $0-100^{\circ} \mathrm{C}$, the formula given by Eisenberg and Kauzmam $(1969, \mathrm{p} .190)$ is used. To calculate the dielectric constant at higher temperatures, the formula given on p.191 of the same book is used. (This foruula is good up to $370^{\circ} \mathrm{C}$.)

Some values of the $\mathrm{pK}_{\mathrm{a}}$ of $\mathrm{MSA}$ and of the Debye-Hückel coefficients are given in the bottom part of Table A3.3.

All single ion activity coefficients were calculated using the extended Debye-HUckel foruula, which is given at the top of Table A3.3. The values of the coefficients $a$ and $b$ used for the various ions are given in the middle part of Table A3.3.

The values of $a$ and $b$ used for $\mathrm{Na}^{+}$and $\mathrm{H}_{3} \mathrm{SiO}_{3}-$ were obtalned from Truesdell and Jones (1974). The values of a used for the other four lons were obtained from Butler (1964, p.434). The value of b used for these last four ions $(0.2)$ is an estimate suggested by the approxinate Davies equation. Unly the activity coefficients for the first three ions are used in SILNUC. The activity coefficients for the citrate ions were used in the reduction of the "fluoride added" data, which was generated using the citrate buffer system.

SILNUC has the ability to convert concentrations in units of grams or woles per liter at $25^{\circ} \mathrm{C}$ to grams or moles per kilogram of water under the assumption that sodiun chloride is the najor solute present. In this calculation it uses the value of the partial molal volume of sodium chloride in water at $25^{\circ} \mathrm{C}$ calculated from the empirical formula

$$
\phi_{V}(\mathrm{~cm})=16.40+2.153 \mathrm{~m}^{1 / 2}
$$

where $\mathrm{m}$ is the molal concentration of sodium chloride. (The calculation is iterative.) This formula is given by Harned and Owen (1958, pp.358-61).

Throughout our data reduction work, it was necessary to calculate the value of $\mathrm{pH}_{\text {now }}$ for the solutions used in our experinents. This is mostly a watter of calculating the sodium ion activity in the solution. The sodium ion activity is, in turn, determined by the sodiun ion concentration and Ionic strength, which vary with the buffer system employed, the Initial silica concentration, the $\mathrm{pH}$, and the added salts (if any). The contributions of the added salts to the sodiun ion concentration and Ionic strength are trivial to calculate. Therefore, the calculation essentially reduces to 
Table A3.3

Formulas and Values for Calculating Activity Coefficients and $\alpha_{\text {sil }}$

The extended Debye-Hückel equation:

$\log \gamma=-A_{D^{2}} I^{1 / 2} /\left(1+a B_{D H} I^{1 / 2}\right)+b I$

The fraction of monomeric silica in ionic form:

$\alpha_{s i 1}=1 /\left[1+\gamma_{s i 1}\right.$ antilog $\left.\left(p K_{s i 1}-p H\right)\right]$

Species

$\mathrm{Na}^{+}$

$\mathrm{H}_{3} \mathrm{SiO}_{4}^{-}$

$\mathrm{F}^{-}$

Citrate ${ }^{-1}$

Citrate ${ }^{-2}$

Citrate ${ }^{-3}$

Temp. $\left({ }^{\circ} \mathrm{C}\right)$

50

75

100

125

150 a

4.

4.

3.

3.

5.

5.

0.075

0 .

0.2

- 0.2

0.2

0.2

b

0.075

0.2


determining the contributions of the buffer system and the added sodium metasilicate and sulfuric acid to the sodiun concentration and lonic strength in the experimental solution that ultimately results.

The buffer stock solution introduces a certain concentration of sodium which varies only with the buffer system that is used. The sodium metasilicate stock solution introduces an amount of sodium that varies in proportion to the InItial dissolved silica concentration. The sulfuric acid that is added to neutralize the sodium metasilicate and adjust the $\mathrm{pH}$ to the desired value introduces an amount of sulfate ion that varies with the concentration of silica, the cholce of baffer system, and the final pH. The choice of buffer system, the Initial silica concentration, and the actual pH of the resulting solution were recorded for each experiment and were known. The amount of sulfuric acld added was not recorded, and had to be calculated.

To calculate the amount of sulfuric acid needed to adjust the $\mathrm{pH}$ to the given value, as well as to determine the contribution of the buffer ions to the Ionic strength, it is necessary to calculate the dissociation equilibria for the buffer compounds. Because the dissociation equilibria of the buffer compounds are effected by the lonic strength, these calculations should, In principle, be done iteratively. In the case of the citrate buffer system; this is a significant effect, and the calculations were done 1teratively, using activity coefficients for the three citrate lons that were calculated using Eqn. (A3.3.1) and the values in Table A3.3. In the case of the maleate and maleate-barbital buffer systems, the details of the buffer dissociation equilibria have relatively little effect on the lonic strength and the sodium lon activity that is finally calculated. Therefore, the activity coefficients of the lons of maleate and barbital were all simply set equal to unity, and Iteration was not needed.

Most of these calculations were done on a programmable desk calculator. The three $\mathrm{pK}_{\mathrm{a}}$ values assumed for citric acid at $50^{\circ} \mathrm{C}$ we re $3.09,4.76$ and 6.48. These were determined from the equilibrium constant values given by Weast and Se1by $(1967, \mathrm{p} \cdot \mathrm{D}-92)$. The three corresponding values assumed at $70^{\circ} \mathrm{C}$ were $3.06,4.76$ and 6.56 . These were quadratically extrapolated from the values at 30,40 and $50^{\circ} \mathrm{C}$ calculated from equilibrium constant values given in the same place. 
For barbital at room temperature, Weast and Selby $(1967$, pp. D-90-91) give the value $\mathrm{pK}_{\mathrm{a}}=7.43$. In our laboratory, we estimated $\mathrm{pK}_{\mathrm{a}}$ for barbital to be about 7.4 at $100^{\circ} \mathrm{C}$, which.is within experimental error of the preceeding value. Therefore, we chose to use the value 7.43 at all temperatures. For the second dissociation of maleic acid at $25^{\circ} \mathrm{C}$, weast and Selby (same place) give $\mathrm{pK}_{\mathrm{a}}=6.07$. We experimentally estimated it to be 6.40 at $100^{\circ} \mathrm{C}$. We chose to use these values at the corresponding temperatures, and values determined by linear interpolation between them at intermediate temperatures.

\section{A3.4 Tables of Selected Experimental Data}

Tables A3.4 thru 15 contain selected kinetic data in very nearly "raw" form. For the most part, the only processing that the data in these Tables has been subjected to is to convert the initial optical density values to molybdate active silica concentration values, and to adjust the time scales so that time zero is at the start of the reaction. In the case of the continuous flow kinetic system data, the tabulated values correspond to digitization points that were manually placed and then "read off" at conve-. nient but otherwise arbitrary intervals along the original continuous curves produced by the chart recorder.

For the most part, the Tables should be self explanatory. However, recall that here, as elsewhere In this Chapter, all concentrations are given in units of grams or moles per liter at room temperature, and not at the actual temperature of the experiment. In the case of the experiments that involved no added salt, this is essentially the same as concentrations in terms of grams or moles per kilogram of water.

The data in Table A3.7 was generated using citrate buffer. The data in all the other Tables here was generated using the barbital-maleate buffer system.

Tables A3.4 thru 6 , contain all of the molecular deposition ("colloid added") rate data that was used to determine the rate of molecular deposition. $\mathrm{k}\left(\mathrm{pH}, \mathrm{pH}_{\mathrm{nom}}\right)$ is the value of $\mathrm{k}_{\mathrm{OH}}(\mathrm{T}) \mathrm{F}\left(\mathrm{pH}, \mathrm{pH}_{\text {nom }}\right)$ directly determined by fitting the data as described in S3.4. "RMS error" is the root-mean-square discrepancy between the empirical and fitted values of the time (which is treated as the 
dependent variable in this context). In all cases, the fits were performed using the trial function described by Eqns. (3.4.4 and 7 ).

Table A3.7 contains the results of one of three sets of "fluoride added" experiments that were performed. (The results of another such set are presented in Fig. 3.12.)

Tables A3.8 to 12 contain all of the homogeneous nucleation data that was used in the data reduction discussed in S3.10. Th1s data 1s also presented (with shifted time scales) in Figures 3.14 to 17.

Tables A3.13 to 15 contain the results of six out of the twelve sets of homogeneous nucleation experiments in which sodium chloride was added to some of the solutions. The data from one of these sets are also presented In Fig. 3.19. The data from a seventh set that is not included in the Tables here are presented in Fig. 3.20. 
TABLE A3.4

Molecu?ar Deposition Data for $50^{\circ} \mathrm{C}$

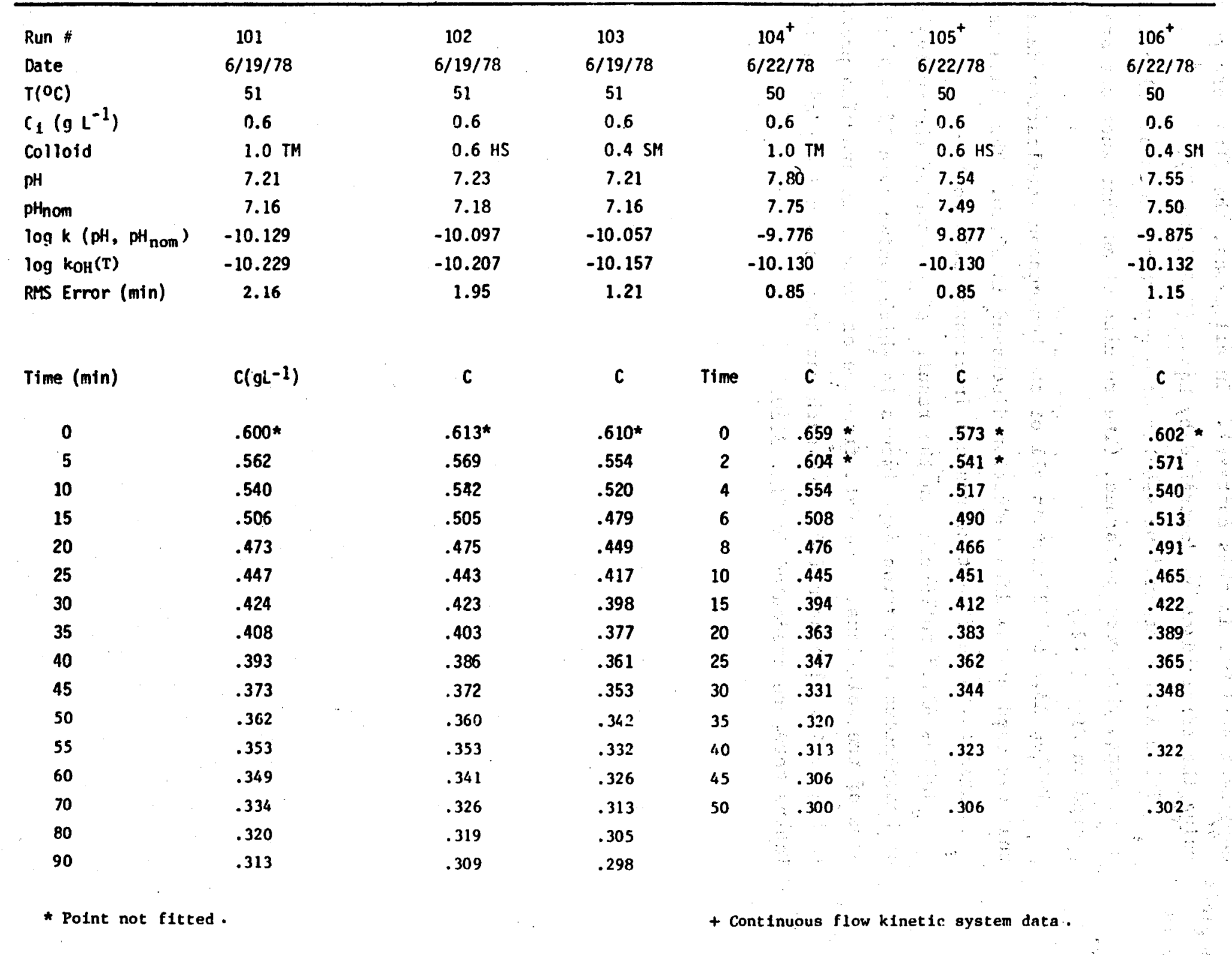




\section{Table A3.5}

Molecular Deposition Data for $75^{\circ} \mathrm{C}$

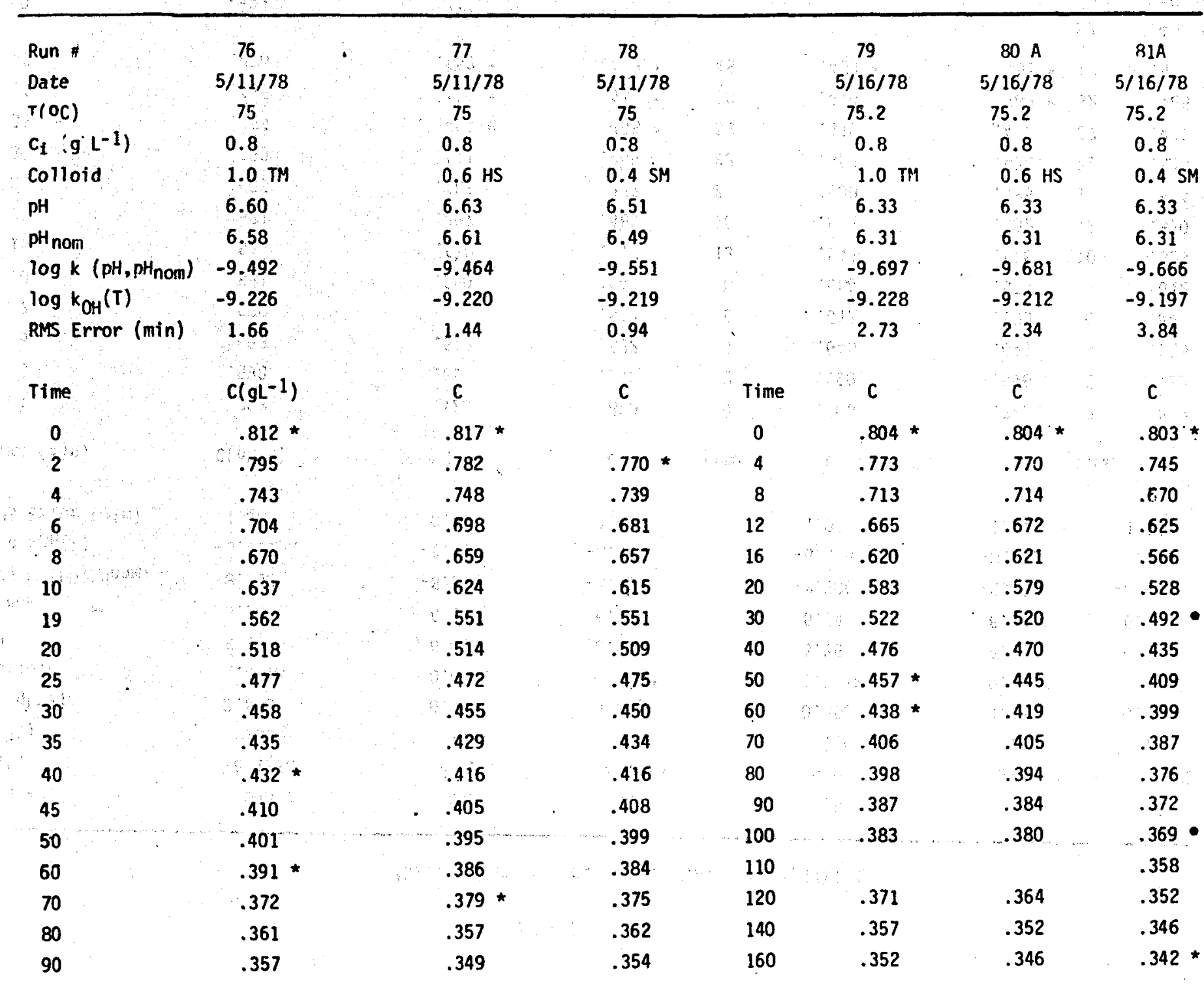

- Point not fitted. 
TABLE $A 3.6$

Molecular Deposition Data for $100^{\circ} \mathrm{C}^{+}$

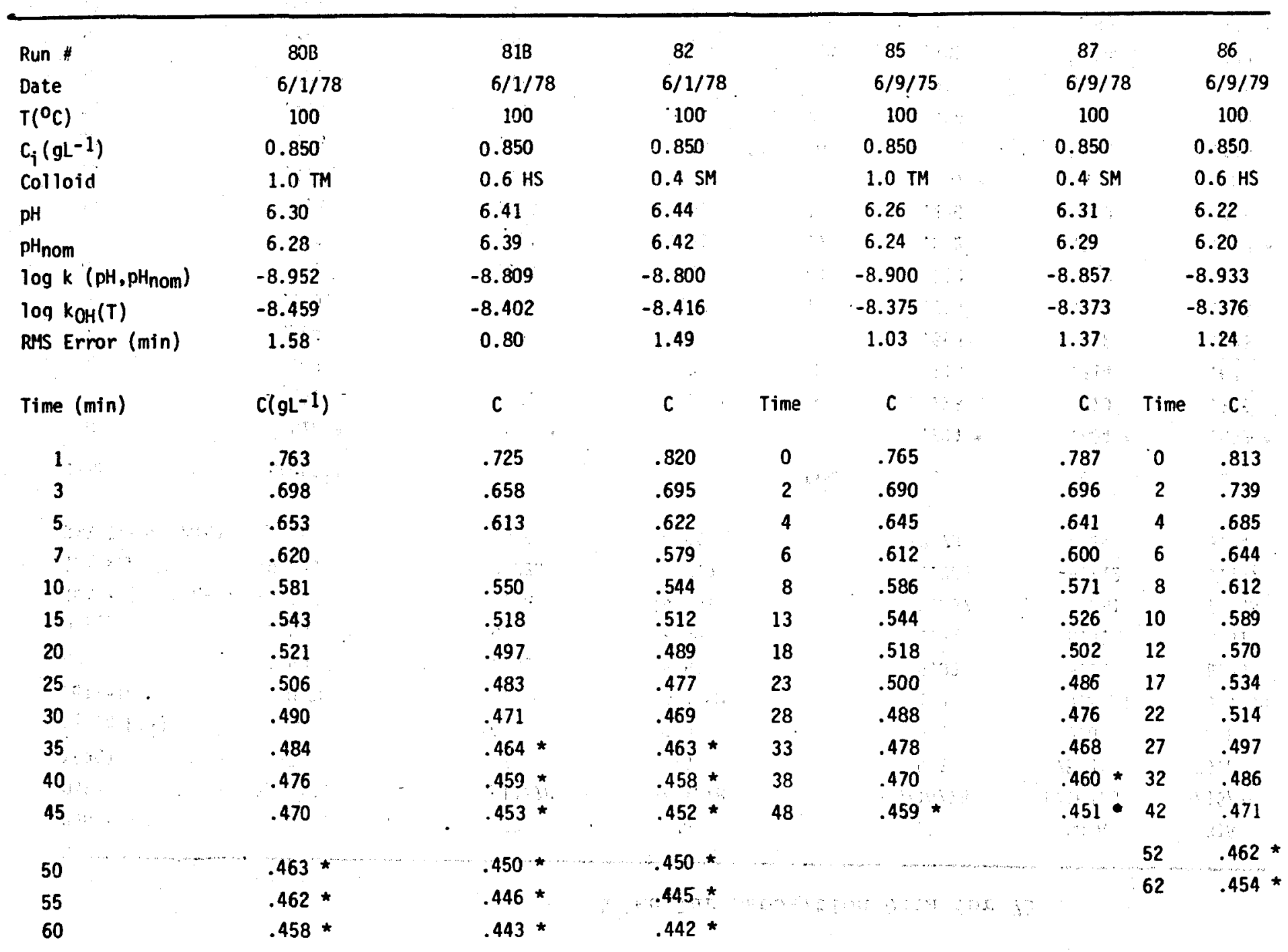

* Point not fitted.

+ All data on this page generated using the continuous flow kinetic system. 
TABLE A3.7

Fluoride Catalysis Data

Homogeneous Nucleation of $70^{\circ} \mathrm{C}$

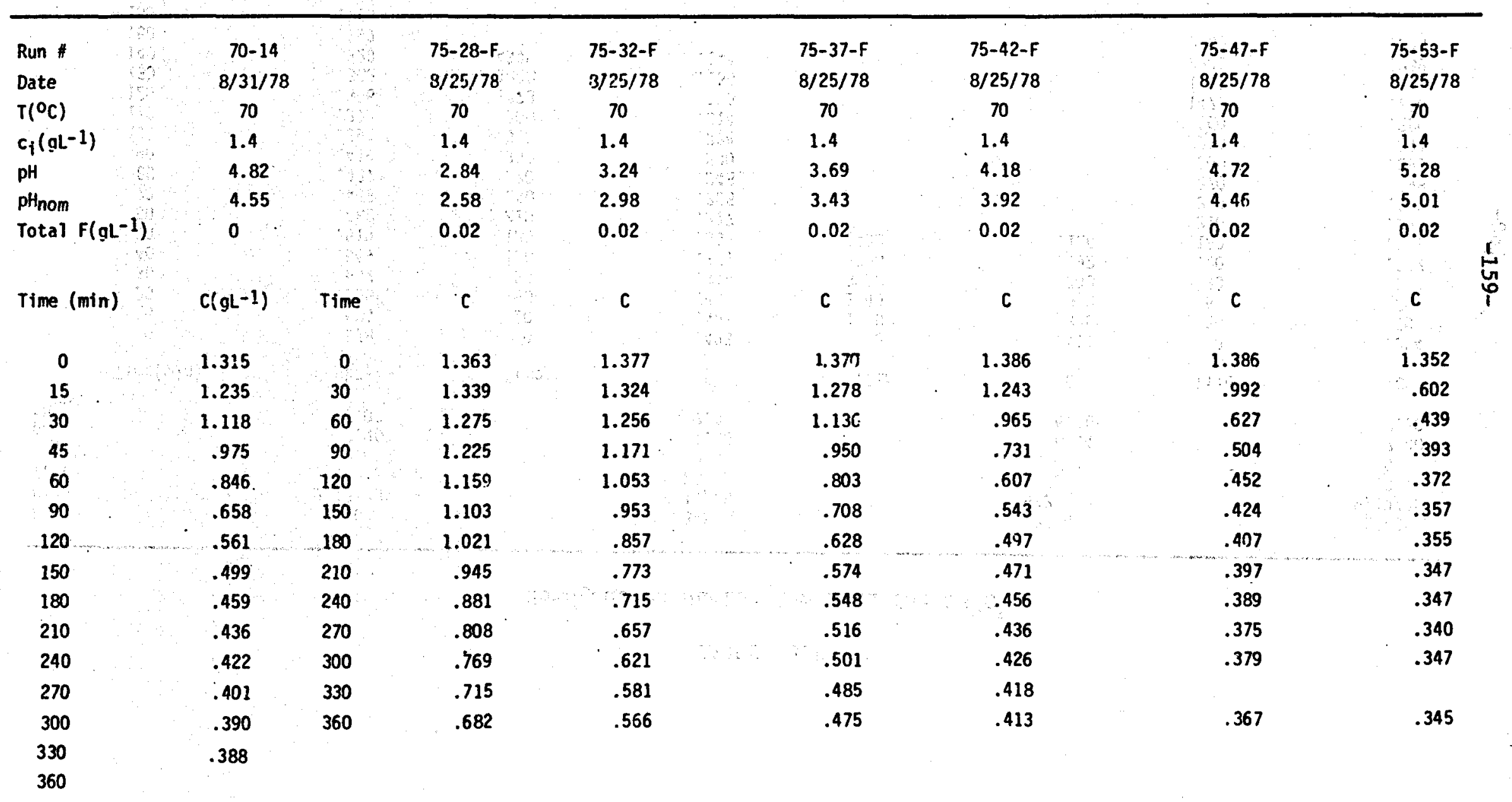


TABLE A3. 8

Homogeneous Nucleation Data for $23^{\circ} \mathrm{C}^{*}$

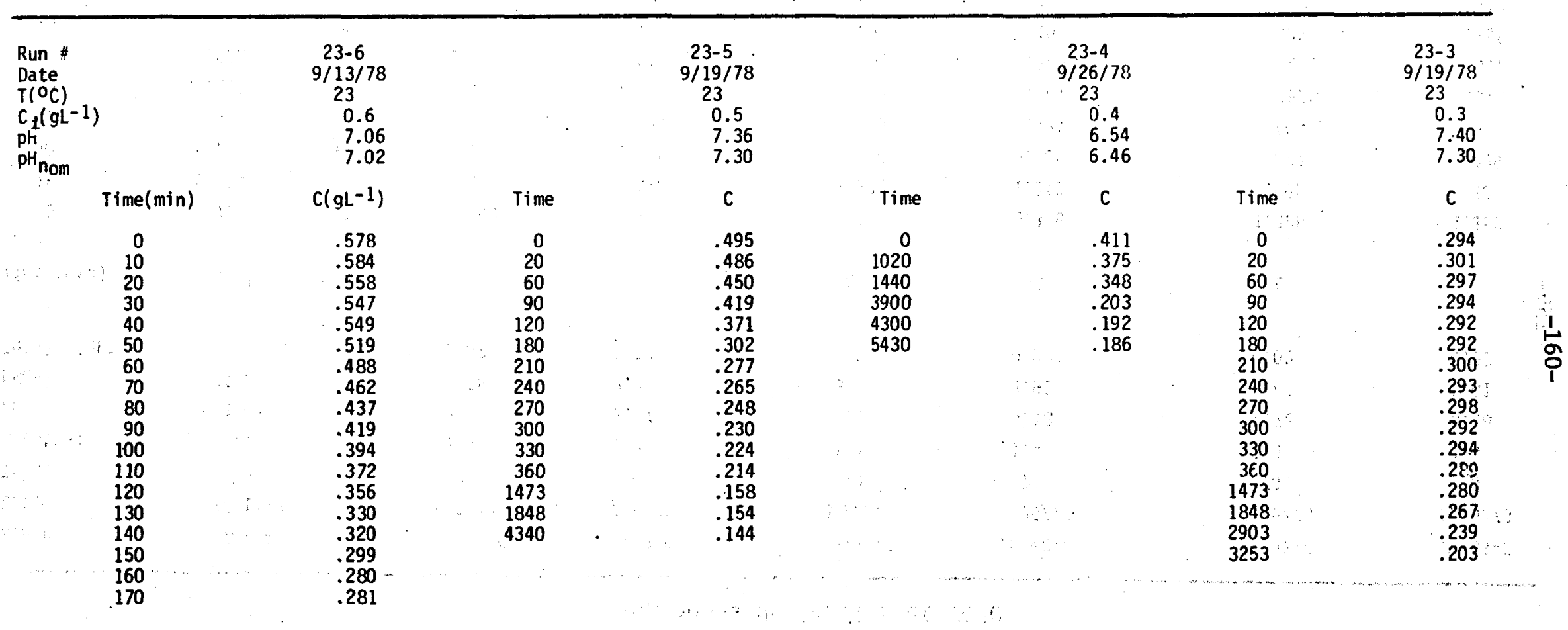

*The data in this table was not fitted with code SILNUC.

$C^{\prime}, \cdots+$ 
TABLE A3.9

Homogeneous Nucleation Data for $30^{\circ} \mathrm{C}$ *

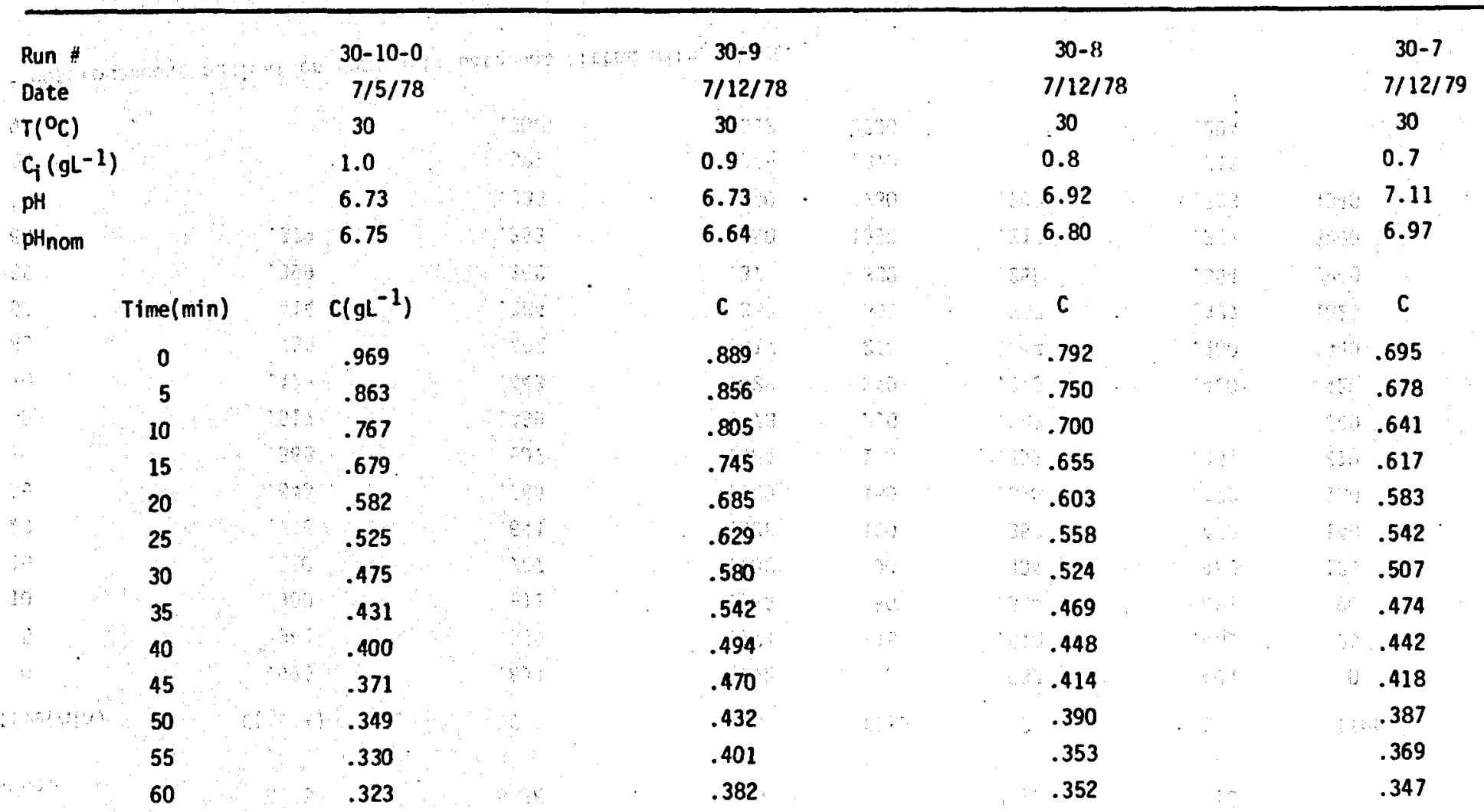

*The data in this table was not fitted with code SILNUC. 
TABLE A3.10

Homogeneous Nucleation Data for $50^{\circ} \mathrm{C}$

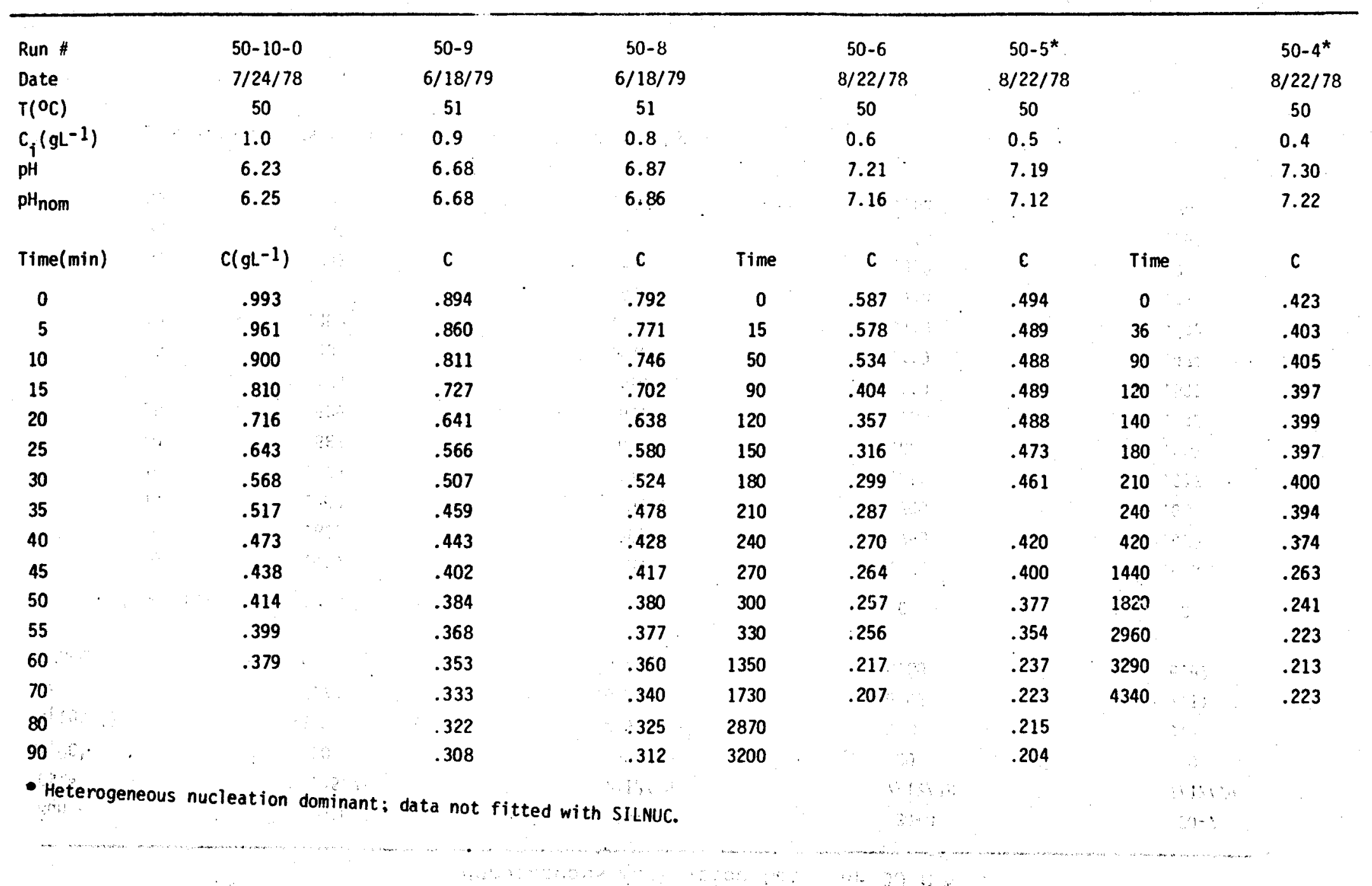

C $\cdots+$ 


\section{TABLE A3.11}

Homogeneous Nucleation Data for $75^{\circ} \mathrm{C}$

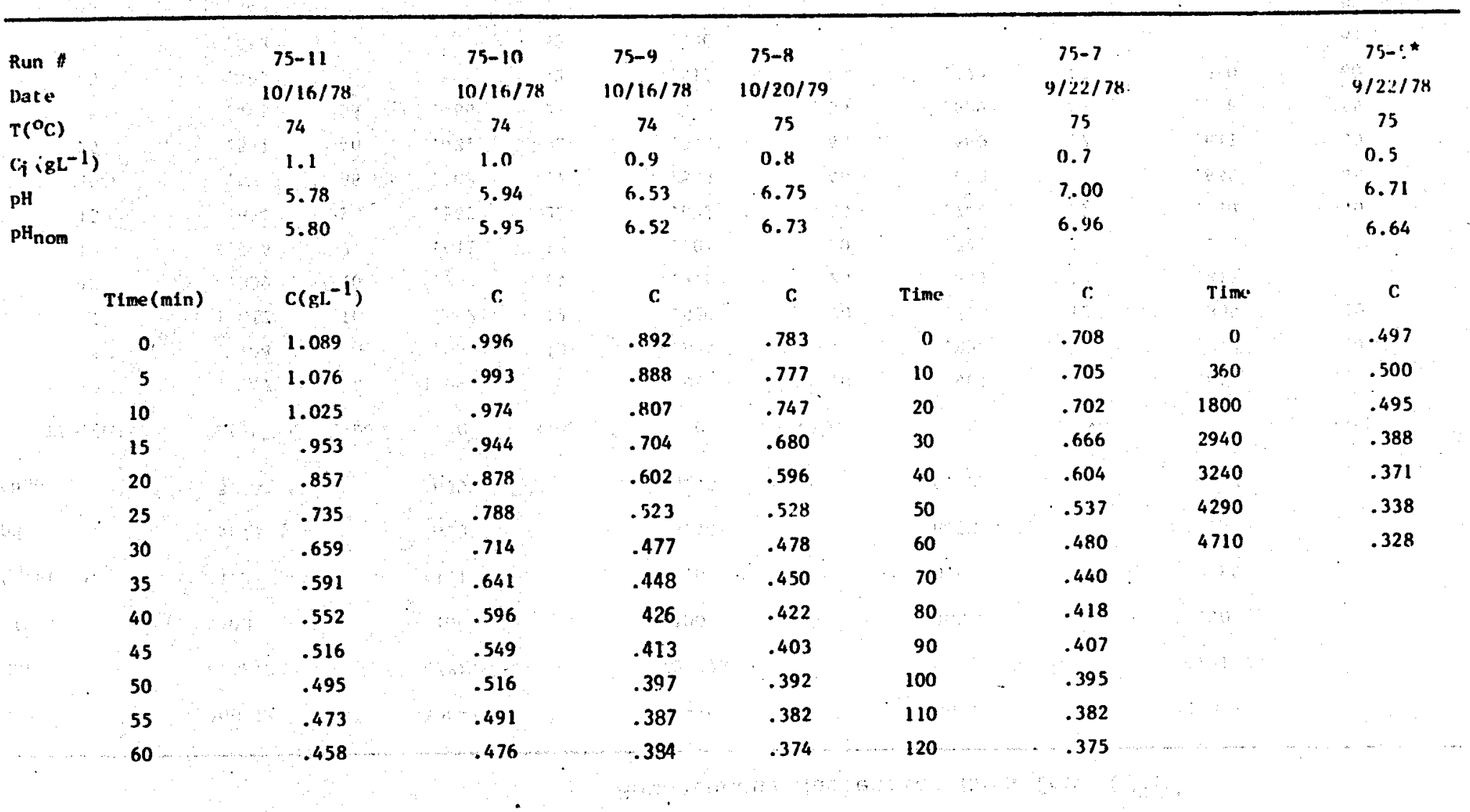

- Hetereogeneous nucleation dominant; data not fitted with SILNUC. 
TABLE $A 3.12$

Homogeneous Nucleation Data for $100^{\circ} \mathrm{C}^{+}$

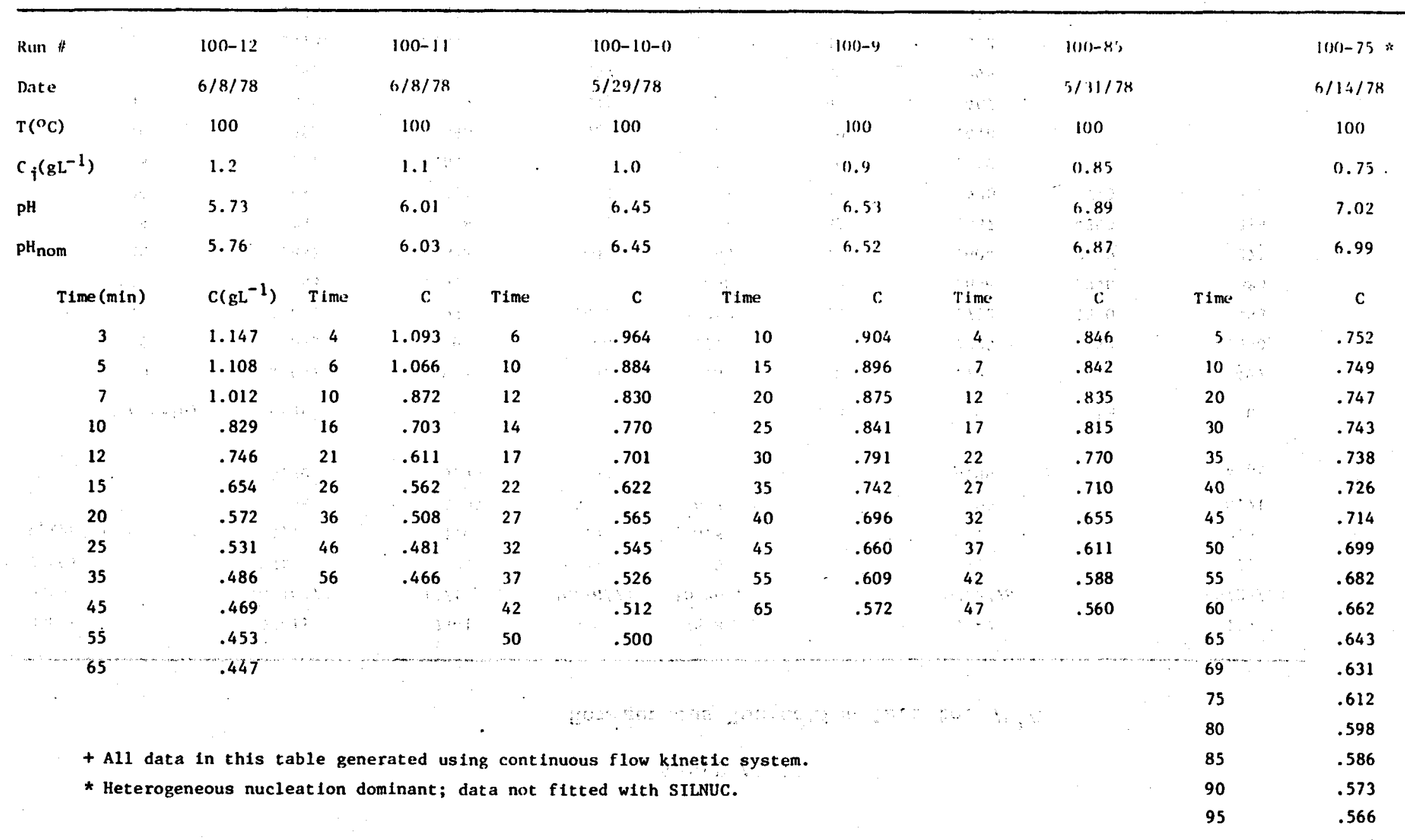


TABLE A3.13

Effect of Sodium Chloride on Homogenous Nucleation

Selected Data for 30 and $100^{\circ} \mathrm{C}$

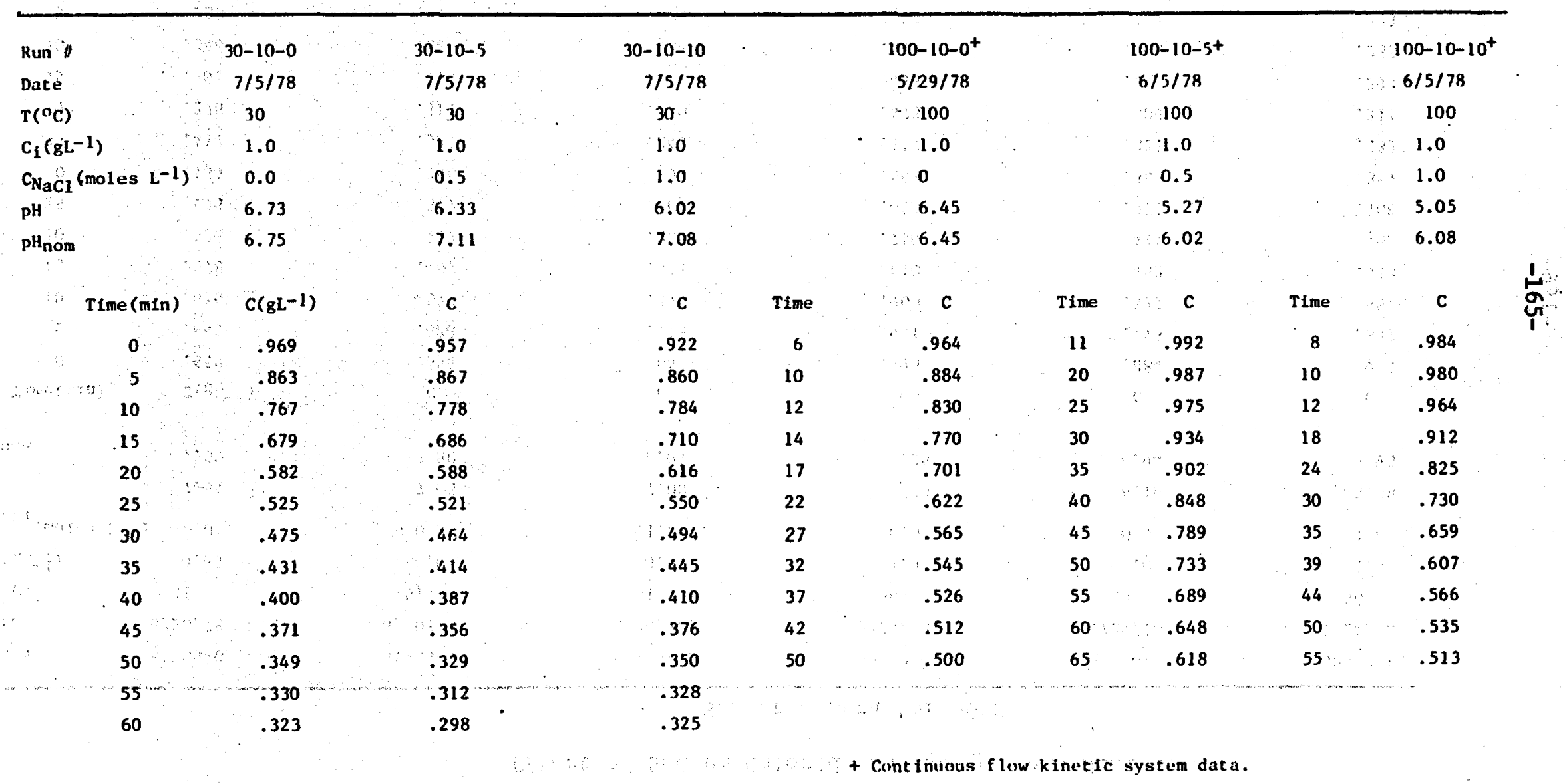


TABLE 3.14

Effect of Sodium Chloride on Homogeneous Nucleation.

Selected Data for $50^{\circ} \mathrm{C}$

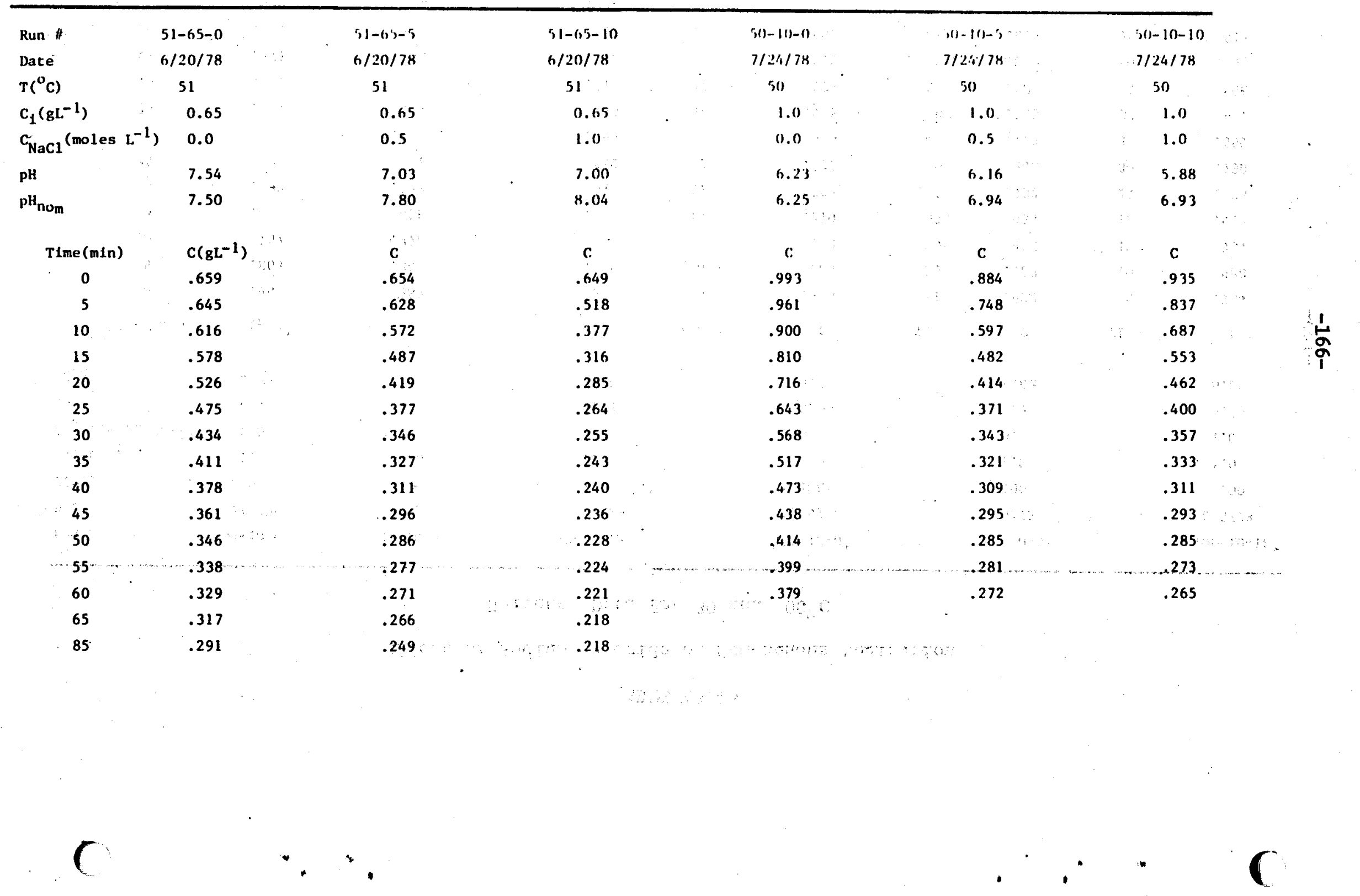


TABLE A3.15

Effect of Sodium Chloride on Homogeneous Nucleation

Selected Data for $75^{\circ} \mathrm{C}$

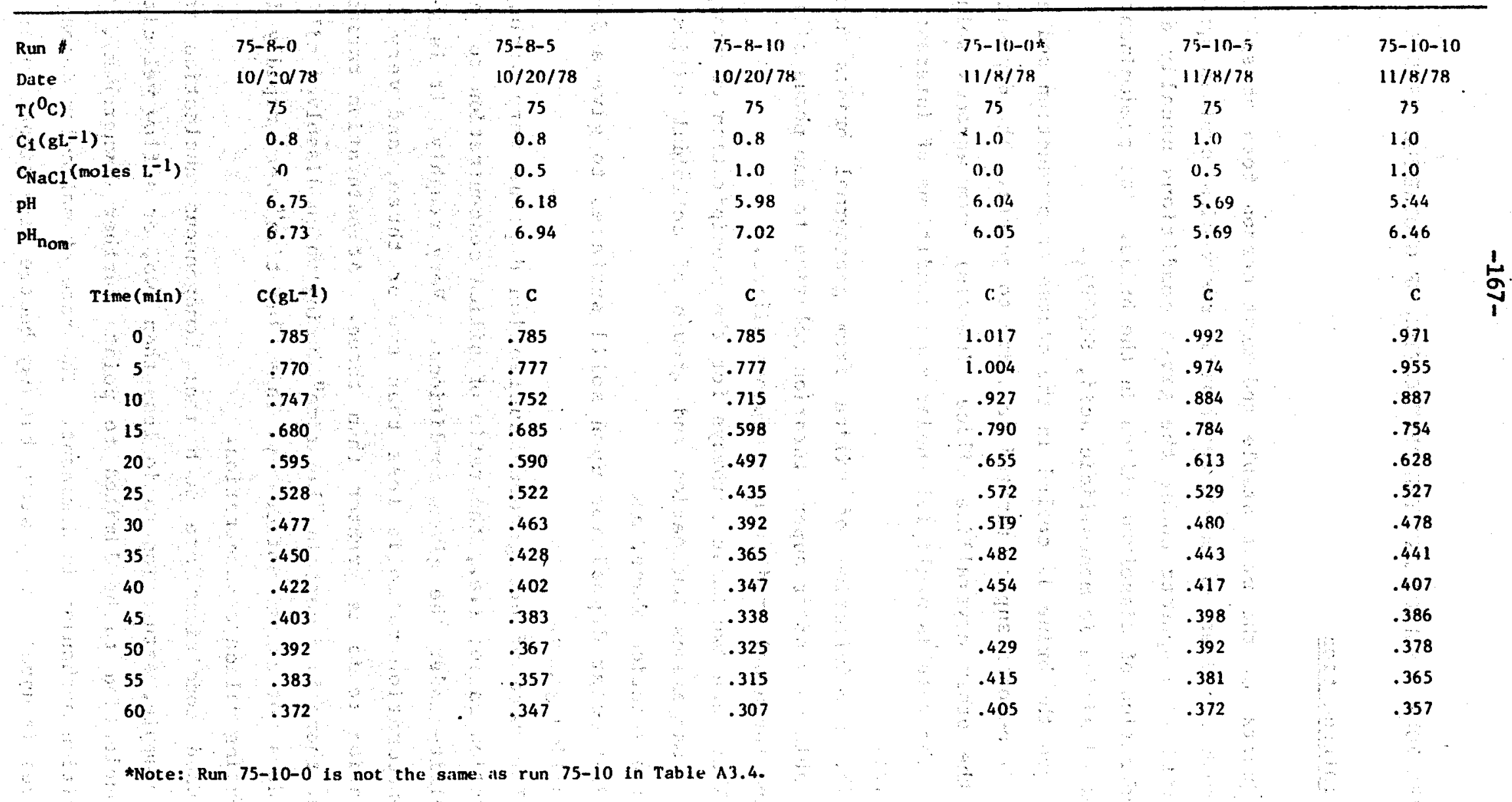


CHAPTER FOUR - SILICA AND THE REINJECTION OF GEOTHERMAL BRINES

\section{S4.1 Introduction}

Quartz is the most stable and most common form of silica in nature. The rock matrices of most geothermal reservoirs contain quartz, and the solubility of quartz under the given conditions usually determines the concentration of dissolved silica in the brine. The predominant form of this dissolved silica is monosilicic acid, $\mathrm{Si(OH)} 4^{\circ}$

When the brine is cooled in the course of production and energy extraction, it becomes supersaturated relative to quartz. The deposition of quartz (and the other crystalline silica minerals) from aqueous solution is very slow. The rate is negligible under most conditions of interest to geothermal practice.

The precipitation of silica from geothermal brines usually becomes noticeable only after supersaturation relative to amorphous silica is attained, and the usual product is amorphous silica.

There are two basic pathways for the precipitation of amorphous silica:

1) Homogeneous nucleation and growth of colloidal silica particles in the solution phase, and

2) Molecular deposition upon solid surfaces to give a dense, compact silica glass.

The rate of homogeneous nucleation depends very strongly upon the saturation ratio; $1 . e .$, the ratio of sillca concentration to the equilibrium solubility under the given conditions. Very roughly, it is very slow when the saturation ratio is less than two or three, and very fast when the saturation ratio is greater than three. At saturation ratios below about three, there is a certain "lag time" during which dissolved silica concentration does not change noticeably. This "lag time" very rapidly increases with decreasing silica concentration.

When conditions are such that homogeneous nucleation is unimportant (for example, saturation ratio less than two, or a flow velocity fast enough for nucleation to be limited to points further downstream), the molecular deposition mechanism is dominant. The rate of molecular deposition on solid surfaces is usually very slow, but may become significant under conditions of high silica concentration and temperature. For example, deposition of vit- 
reous silica at a rate of about $1 \mathrm{~mm} / \mathrm{year}$ has been observed in some of the spent brine pipes at Cerro Prieto.

The above factors tend to restrict the amorphous sillca precipitation problem to situations in which there is a large decrease in brine temperature. In practice, this means that silica precipitation is likely to be a problem only if the reservolr temperature is above about $240 \circ \mathrm{C}$. This is illustrated In Table 4.1 .

For a more detalled discussion of the kinetics of precipitation of amorphous silica, see Chapter III of this report.

\section{S4.2 Types of S1lica Deposits}

The deposition of vitreous silica on solld surfaces by the molecular deposition process has already been mentioned. The deposition rate is always slow, but the material is almost indestructible once formed. Slow chemical dissolution with hot HF or caustic appears to be the only way to remove $1 t$.

The first step in the conversion of colloldal silica to solid or semisolid deposits is flocculation or coagulation followed by settling or chemically equivalent adhesion to solid surfaces.

The rate and extent of coagulation of colloldal silica is determined mostly by $\mathrm{pH}$ and salinity. Above about $\mathrm{pH}$, the collold becomes less stable with increasing $\mathrm{pH}$. Increasing salinity also enhances coagulation. DI- and polyvalent cations (In practice, usually calcium) are particularly effective in this regard, especlally at pH values above about 7.5. Also, larger particles are more strongly coagulated by divalent cations. This is probably not true of coagulation by univalent cations.

Unfortunately, available data are Inadequate to allow sllica coagulation to be predicted quantitatively. However, good, semiquantitative data regardIng "critical coagulating concentrations" at room temperature are available In the 11terature. (See Allen and Matifevil, 1969, 1970, 1971, Matijević, 1973, and Iier, 1975 and 1976).

The conversion of electrostatically coagulated colloldal silica to an adherent gel or solid scale Involves cementation of the particles by molecular deposition between them. The rate of this chemical cementation process depends upon the concentration of dissolved silica (among other 
factors). When the colloidal particles are approximately in equilibrium with dissolved silfca, cementation is slow. Under these conditions, cementation involves dissolution of silica from the particles and its redeposition at the interparticle contact points. The only driving force for this process is the difference of surface curvature. Massive adherent deposits will form only at points where hydrodynamic conditions allow the precipitate to settle out and rema in und isturbed.

Falrly hard solid deposits may form at a moderate rate (centimeters per year) where both colloidal silica and substantially supersaturated dissolved silica are present. Cementation is relatively fast under these conditions. Sometimes, when the dissolved silica concentration is high enough and the deposit remains in good contact with the brine, cementation can proceed to the point of producing a glasslike material.

S4.3 Possible Mechantsm for Post-Reinfection Plugging by Silica

Injection of brine can damage the receiving formation if the brine is supersaturated with silica or contains collotdal silica. Any brine that is supersaturated relative to amorphous silica will deposit vitreous silica on solid surfaces at a rate determined by the dissolved silica concentration, temperature, and other variables. The rate may be negligible (the usual case at moderate concentrations and temperatures), or it may not be. What constitutes a negligible rate will depend on the physical properties of the reservolr. For example, $0.02 \mathrm{~mm} /$ year would be negligible if the injected fluid were going into a $4 \mathrm{~m}$ fracture. It would be catastrophic if the rock had pore permeabllity only and an average pore size of $0.01 \mathrm{~mm}$.

Note that the rate of molecular deposition is determined only by temperature, concentration and other chemical variables, and is not affected by the hydrodynamic state of the fluid. This means that deposition will continue until the supersaturation is reduced by some combination of concentration decline and reheating.

If brine that contains a floc is reinjected, the floc will accummulate In any place where the brine enters pores or cracks fine enough to filter the floc out. These places will eventually be plugged. If pore permeability is dominant, the plugging will take place right at the wellbore where it will do the most harm most rapidly. (However, damage at the wellbore is also 
the easiest to undo by treating the well with caustic or HF.) In a fractured reservoir, the floc may finally come to rest so far from the infector that no significant injectivity decline will ever be observed. Also, if the floc is not deposited by the time that the brine is reheated, it will partially or completely redissolve.

If, In addition to floc, the brine also contains enough dissolved silica to cause rapid cementation, the spatial distribution of the damage w111 be similar, but the damage will be worse and harder to undo. Fortunately, significant supersaturation cannot persist for long in the presence of large colloid loadings, and this fact will tend to reduce the extent of cementation further back into the formation.

It must be considered that reaction of the brine with reservoir materials could destabilize the collold and accelerate deposition. For example, injecting brine that is undersaturated with calcite into a calcite bearing formation would cause the $\mathrm{pH}$ and calcium content of the brine to increase, thereby destabilizing the colloid.

Finally, a collold-free brine with high enough supersaturation could undergo homogeneous nucleation post-reinjection. Whether or not homogeneous nucleation actually occurred would be determined by whether competing processes such as molecular deposition, heterogeneous nucleation or reheating could reduce the silica supersaturation enough to prevent 1t. Homogeneous nucleation in the formation could involve rapid cementation of the collotd produced because of the inevitability of passing through a stage of simultaneous substantial collofd loading and substantlal silica supersaturation. The extent of damage would again depend on rate and the physical properties of the reservior. Greater supersaturation would cause homogeneous nucleation nearer to the wellbore, and therefore, would be more $11 k e l y$ to cause serious damage. It remains to be considered whether flow through a porous medium can inhibit homogeneous nucleation by removing newly nucleated particles from the brine by adhesion to the rock surfaces before they grow to significant size. 
S4.4 Brine Treatment for Silica Control

A salient characteristic of geothermal energy conversion processes is that they involve large mass flows. Typically, about $10 \mathrm{~kg}$ of native or flash steam is required per kwhr generated, and 20 to $40 \mathrm{~kg}$ of spent brine must be disposed of. This means that a brine treatment cost of more than $10 \mathrm{E}-5$ SUS/kg of brine is significant, and a cost of more than about $10 \mathrm{E}-4 \$ \mathrm{US} / \mathrm{kg}$ is unlikely to be acceptable. This means that brine treatments must be simple, and that chemicals added in concentrations greater than a few parts per million must be very cheap. Also, the sheer volume of brine to be treated can render a process impractical because of unacceptably large brine storage or chemical delivery or handling requirements. Finally, to be commercially viable, the overall energy extraction and conversion system must be as cheap, reliable, and easy to operate as other types of electric powerplants.

With the above considerations in mind, we proceed to review possible approaches to avolding silica related problems in reinjection.

Four distinct approaches have been developed for the control of silica precipitation from geothermal brines:

\section{1) Avoidance or minimization of silica supersaturation}

Supersaturation can be avoided by preventing cooling of brine to below the saturation temperature of amorphous silica for the given concentration of dissolved silica. This is the only completely safe and sure, simple approach to the problem that is available, and is a matter of clever process design for energy conversion rather than actual brine treatment. The substantial difference between the solubility of quartz, which determines the Initial silica concentration in the brine, and that of amorphous silica makes the goal attainable in many cases (see Table 4.1). A successful practical demonstration of this approach will be discussed in the following section.

Increasing brine $\mathrm{pH}$ also increases the solubility of amorphous silica by causing partial dissociation of monosilicic acid. This effect becomes significant above about pH 7.5. Adding enough base to cause a large increase in solubility would probably be impractical in most cases besause of the large amounts that, would be required (as much as several milliequivalents/kg brine). However, in some cases, a small increase in solubility may be 
desirable and practically attainable. Also; some unmodified flashed brines have $\mathrm{pH}$ values high enough for this effect to be significant; for example, the brines at East Mesa (California) are of this type. This should, of course, be taken into consideration in interpreting brine chemistry and devising brine treatments.

Under some circumstances a saturation ratio somewhat in excess of unity may give homogeneous nucleation and molecular deposition rates low enough to be practically insignificant. This is particularly likely at temperatures below $100^{\circ} \mathrm{C}$. This possibility should be carefully considered using available kinetic data (our own work elsewhere in this report) when designing for a saturation ratio of untty or lower appears impractical for some reason.

2) Kinetic inhibition of molecular deposition and nucleation

The rates of all silica polymerization processes decrease with decreasIng $\mathrm{pH}$. Therefore, an obvious brine treatment for the purpose of slowing down silica precipitation, although not actually stopping $1 t$, is to acidify the brine. Alternatively, the $\mathrm{pH}$ may be kept from rising by avoiding flashing the brine, as in a binary cycle with downhole pumped brine production. Another effect of lowering $\mathrm{pH}$ is to Inhibit the coagulation of colloidal silica. This may or may not be desirable in a given instance.

Lowering $\mathrm{pH}$ or keeping it from rising may also decrease or eliminate the precipitation of calcium carbonate.

Most discussions of this brine treatment assume the use of hydrochloric acid. Sulfuric acid is cheaper, but may cause or aggravate the precipitation of calcium, strontium or barlum sulfate. However, this possibility should be carefully evaluated on a case by case basis, and the use of sulfuric acid should not be rejected out of hand because of 1t. The cheapest acid of all is the carbon dloxide contained in geothermal condenser off-gas. This additive $1 \mathrm{~s}$ avallable at no cost, but its ut1lization would require large and potentially complicated and expensive gas-liquid contact equipment. Also, carbon dioxide would not be able to reduce the $\mathrm{pH}$ to much below 6 , and the use of condenser off-gas would inevitably introduce some atmospheric oxygen Into the brine. oxygen could aggravate corrosion problems or cause the precipitation of ferric hydroxide or similar substances.

Workers at the Lawrence Livermore Laboratory have extensively evaluated the use of hydrochloric acid addition for silica control at Niland (Austin, 
et $a 1 ., 1977)$. In that particular case, the estimated cost of brine acidification by addition of 200 ppm HCl was marginally acceptable ( 1 or 2 mils /kwhr). Prohibitive corrosion problems were ultimately responsible for the abandonment of the concept. Also, it was found that pumping the acidified brine through sandstone cores dissolved the calcite in the matrix which led to serious plugging with loosened matrix materlal (Piwinski and Netherton, 1977). All in all, brine acidification proved impractlal at Niland. This may or may not prove to be the case elsewhere.

3) "Aging" the brine to convert dissolved silica to colloidal silica

This process has been demonstrated on a commercial scale at the otake plant in Japan (Yanagase, et al., 1970), and on a pllot plant scale at Ahuachapán in E1 Salvador (Cuéllar, 1975) and at Wairake1 and Broadlands in New Zealand (Rothbaum and Anderton, 1975).

At Otake and Ahuachapán the desired goal was reduction of the rate of scaling in surface waste brine disposal pipes and canals. In both places, untreated brine delivered to these conduits apparently contained both colloldal silica and a substantial excess of dissolved silica: Rapid accumulation of hard scale of the cemented colloidal aggregate type was observed. "Aging" the brine by retaining it in a suitable tank for a period of time was tried in both cases in a consclous attempt to convert as much silica as possible to a relatively nonadhesive "polymeric form", or, In other words, to reduce the dissolved silica concentration by allowing time for its conversion to colloidal silica.

The experiments succeeded in both cases, and a commercial scale "brine aging pond"was actually constructed and put in to routine operation at Otake. In both places, the rate of scale accumulation in the brine disposal system was greatly diminished. Scanning electron micrographs of scale specimens from Otake clearly show that the scale at the outlet of the "aging pond" is a fluffy, weakly cemented floc-like materlal. Cuellar comments on the accumulation of about 14.5 metric tons of scale in the aging pond, but notes that: "Almost all deposits in the tank are very light, porous, and easily removed."

In both cases, only a fraction of the colloidal silica in the brine was deposited in the "aging pond" or disposal system. Most of it was apparently carried away by the brine in metastable or weakly flocculated, nonadhesive form. 
In New zealand, brine aging has been experimented with as the first step of a more complicated course of brine treatment. The primary purpose there was to convert dissolved silica, which cannot be removed by coagulation, to colloidal silica, which can. The desired goal was attained there as well.

The time needed for the dissolved silica concentration to drop into the range of slowly decreasing "near sceady state" values will vary widely with the chemical parameters of the brine. In the cited Instances, adequate agling times were found to be as follows: Broadlands, 30 minutes, Ahuachapán, 45 minutes, Otake, 1 hour, and Wairakei, $21 / 2$ hours.

In general, the time required w1ll decrease with increasing inftial supersaturation, temperature, $\mathrm{pH}$ and salinity. The reader is referred to the data presented by us elsewhere in this report in this regard.

A varlety of different physical configurations for the brine aging vessel seem to wrik well. The maln thing that the apparati seem to have in common is that they all provide approximately plug flow reactor conditions. We refer the reader to the original papers and to the review by Phillips, et al. (1977) for further deta1ls. Our only firm recommendation is that the aging vessel have a cover of some sort in order to prevent contact with air and to minimize heat loss.

\section{4) Removal of colloldal silica by coagulation and settling}

In most cases, it will probably be necessary to remove any colloldal silica that may be present in the brine before reinjection. Similar needs are common in other circumstances, and a body of cleverly devised technology exists and is available for this purpose. Technical evolution in this area has largely consisted of careful application of existing knowledge to the particular circumstances of geothermal development in specific areas.

In New Zealand, the goal was to develop a practical treatment which would yleld brine Innocuous enough to delfver to nonelectrical users or to dispose of in fresh surface waters without envirommental risk. Primarily, this required the removal of colloldal silica and arsenic, and secondarily, some reduction of dissolved silica to below the levels attainable by aging processes described above. The addition of 400 to $700 \mathrm{ppm}$ of calcium oxide (unslaked IIme; $\mathrm{CaO}$ ) and about $10 \mathrm{ppm}$ of sodlum hypochlorlte (NaC10) to the aged brine followed by settling was found to remove all of the colloidal silica and most of the arsenic and dissolved silica (Rothbaum and Anderton, 
1975).- The product is a high surface area mixture of colloidal amorphous silica and amorphous calcium silicate which might have some practical use.

Removal of colloidal silica alone is not explicitiy discussed by Rothbaum and Anderton, but the data presented by them suggest that about 100 ppm CaO alone may be adequate for this purpose. This relatively large amount appears to be needed because the New Zealand brines are of rather low salinity and, in particular, contain 11ttle calclum. Therefore, a large increase in $\mathrm{pH}$ and/or calcium ion concentration is needed to flocculate the silica in them.

We note that increasing the $\mathrm{pH}$ and/or calcium ion concentration may cause or aggravate calcium carbonate precipitation elther at the surface or post-reinjection. This is particularly to be expected in the case of a very low calcium and high bicarbonate brine like that at Broadlands... This possibility must be carefully considered on a case by case basis if lime addition is being considered.

Removal of colloidal silica from the brine at Niland (California) has been demonstrated by Quong, et al. (1978) on a small pilot plant scale. The suspended solids in the flashed brine at Niland consist mostly of colloidal amorphous silica, but contain smaller amounts of other substances as well. However, the silica dominates the overall behavior of the precipitate: This brine is exceptionally saline and has a particularly high calcium content, but the $\mathrm{pH}$ is only about 5.5. This atypically low $\mathrm{pH}$ value causes the decline in dissolved silica to be slow, and the rate and extent of coagulation of colloidal silica to be small. Raising the pH helps matters considerably, but causes the supernatant to cloud with ferric hydroxide following precipitation. This behavior is due to the very high iron concentration in Niland brines. (This effect might be avoldable by avoiding contact with air, but this is not discussed in the cited paper.) All in all, Niland is a rather difficult case.

The process proposed is as follows:

First, a few ppm of the coagulant aid are added to the brine and it is rapidy stirred for five minutes. Then it enters the clarifier. This device has a large amount of slowly stirred coagulated silica sludge at the bottom. The brine slowly flows through the sludge, and as it does, the very high density of coagulated colloidal silica in the sludge rapidly reduces 
the dissolved silica concentration in the brine, as well as coagulating and retaining most of colloldal silica. The brine then flows up and out of the sludge blanket at about $0.25 \mathrm{~mm} / \mathrm{s}$. This is the maximum velocity consistent with good separation of the clarified brine $f:$ on the sludge through gravitational settling of the latter. The clarifier overflow still contains an unacceptably high concentration of suspended solids ( $44 \mathrm{ppm}$ in the best performance reported). However, a final, sand bed filtration reduces this to below $5 \mathrm{ppm}$, which is considered good enough for reinjection.

Laboratory simulation work on Cerro Prieto brines (Weres and Tsao, 1980) suggests that a much simpler treatment would suffice there: $10-20$ minutes aging, addition of 20-30 ppm CaO, 5 minutes of vigorous stirring, and separation of precipitate from brine in a settling tank. The resulting precipitate settles at about $1 \mathrm{~mm} / \mathrm{s}$ and the "clarified brine" contains less than 4 ppm suspended silica.

That such a simple process appears adequate for Cerro Prieto is fortuitous happenstance. As 1 t happens, when flashed down to $1000^{\circ}$, Cerro Prieto brine is in a chemical state conducive to very fast colloldal silica nucleation and dissolved silica decline. The resulting colloidal silica is only moderately flocculated in the unmodified brine, and does not begin to settle until the "aging process" has run 1ts course. However, it is near to the $\mathrm{pH}$ range of strong flocculation. Increasing the $\mathrm{pH}$ by about one-half unit by adding a small amount of base ( $\mathrm{CaO}$ is the cheapest) causes very rapid and essentially complete flocculation.

The brine treatment processes reviewed under this subheading are obviously all variations on the same basic concept, but vary greatly in 1 ts concrete realization. This varlation is largely due to the different brine chemistries in the different areas. This clearly demonstrates that an "off-the-shelf" approach is not appropriate in the area of geothermal chemistry. Rather, the process must be tallored to the particular characteristics of the given brine.

\subsection{Fleld Experience with Geothermal Reinjection}

The only discussions of large scale reinjection of geothermal brines in the literature deal with Ahuachapán (Einarsson, et.al., 1975; Cuéllar, 
1975) and Otake (Kubota and Aosaki, 1975).

At Ahuachapan, the brine was initially saturated with quartz at about $240^{\circ} \mathrm{C}$, and was flashed down to about $155^{\circ} \mathrm{C}$ and reinjected at $152^{\circ} \mathrm{C}$. Therefore, it was slightly undersaturated with amorphous silica at injection (see Table 4.1). As predicted, there was no permeability reduction or scaling of any sort observed over the duration of the injection experiments, which totaled almost $2 \mathrm{E} 6 \mathrm{~m}^{3}$ of brine. This is the classic successful demonstration of the supersaturation avoidance approach to silica precipitation control.

Routine reinjection of spent brine from the geothermal development at otake began in 1972. As of 1975, 8E6 tonnes had been injected into three wells. The receiving interval was a highly permeable stratum of tuff breccias $300 \mathrm{~m}$ thick. Over the three years, a slow decrease in injectivity of the three wells was observed. For example, with the water level at the wellhead, reinjection well No. 1 took 310 tonnes/hr at the beginning of injection, but only 120 tonnes/hr in 1975. Scale was deposited throughout the length of this well. At the wellhead, the scale was about $25 \mathrm{~mm}$ thick after three years and consisted mostly of silica.

Unfortunately, nothing is said about the preinjection treatment that the brine received (if any), or even about the brine temperature. However, Yanagase, et al. (1970) reported that a full field capacity brine aging pond was in routine operation as of 1970. Therefore, it seems likely that the injected brine was being treated in this way. This means that the brine probably contained about $200 \mathrm{ppm}$ of metastable colloidal silica and was supersaturated with amorphous silica by a few percent. If so, the reported injectivity decrease was probably due to slow deposition and cementation of colloidal amorphous silica both near and at some distance from the wellbore.

The injectability of the spent brine at Niland has been extensively studied at that site by laboratory methods as well some actual injection tests (Owens, et a1., 1977; Netherton and Owen, 1978). Injection tests showed that untreated brine quickly and completely plugged up the pore permeability of the receiving formation right at the wellbore. However, injection was not stopped by this plugging because a large fracture zone continued to take the brine. The membrane filter test method commonly used in petroleum practice to evaluate the injectability of waters (Barkman and 
Davidson, 1972) was adapted to geothermal use and successfully applied. Its results agreed well with those of the injection experiments. Also, membrane filtration experiments clearly demonstrated the mechanism of deposition of cemented colloidal aggregates from brine that contained both colloidal and supersaturated dissolved silica. Core-flushing test methods and equipment for evaluating infectability were developed, applied, and described as well.

\section{S4.6 Outstanding Research Needs}

A basic need in all areas of geothermal chemistry is that field data of varlous sorts be carefully collected and exhaustively analyzed. This does not necessarily mean that grandiose new experimental programs need to be started. Rather, ongoing test and operational activities should be planned with the participation of chemists, and provisions should be made for adequate and well defined sampling, analysis, and description. The ultimate product of this work should be a serles of well documented case studies that describe and explain what actually happens. Thorough characterization of all precipitated solids must be included in such studies, and every effort should be made to compare and reconcile the predictions of theoretical and laboratory studies with actual field experience.

The major, outstanding need in the area of silica chemistry is a capability to quantitatively explain and predict the colloidal stability and coagulation behavior of colloidal amorphous silica, and 1 ts cementation following coagulation. Combined with existing knowledge of the chemical kinetics of amorphous silica precipitation, this information would go a long way towards systematizing the design of preinjection brine treatment processes.

A related need is a better understanding of the precipitation of amorphous silicate materials. Work in this areas should start with careful characterization of observed precipitates, and move toward the sort of predictive capability that exists for pure amorphous silica.

A need specific to the study of reinjection is a better understanding of exactly how and where precipitation and plugging occur, and what their effects on formation properties actually are. 
Table 4.1

Silica Concentrations, Saturation Ratios, and Deposition Rates Resulting from Flashing Water Initially Saturated with Quartz

\begin{tabular}{|c|c|c|c|c|c|c|}
\hline \multicolumn{2}{|c|}{$\begin{array}{l}\text { Init. } t\left({ }^{\circ} \mathrm{C}\right) \\
\text { Solub. Quartz(ppm) }\end{array}$} & $\begin{array}{l}200 \\
297\end{array}$ & $\begin{array}{l}220 \\
375\end{array}$ & $\begin{array}{l}240 \\
440\end{array}$ & $\begin{array}{l}270 \\
521\end{array}$ & $\begin{array}{l}300 \\
598\end{array}$ \\
\hline $\begin{array}{l}\text { Final } \\
t\left({ }^{\circ} \mathrm{C}\right)\end{array}$ & $\begin{array}{l}\text { Solub. } \\
\text { Am.Sil. }(\mathrm{ppm})\end{array}$ & & & & & \\
\hline 70 & 245 & $\begin{array}{c}1.315 * \\
390 \\
1.594 \\
0.010\end{array}$ & $\begin{array}{r}1.387 \\
520 \\
2.123 \\
0.058\end{array}$ & $\begin{array}{r}1.469 \\
646 \\
2.638 \\
0.145\end{array}$ & $\begin{array}{r}1.619 \\
844 \\
3.443 \\
0.30\end{array}$ & $\begin{array}{l}2.004 \\
1,198 \\
4.891 \\
0.62\end{array}$ \\
\hline 100 & 364 & $\begin{array}{r}1.238 \\
368 \\
1.010 \\
0.0003\end{array}$ & $\begin{array}{r}1.303 \\
489 \\
1.342 \\
0.029\end{array}$ & $\begin{array}{r}1.377 \\
606 \\
1.664 \\
0.13\end{array}$ & $\begin{array}{l}1.514 \\
789 \\
2.167 \\
0.64\end{array}$ & $\begin{array}{l}1.859 \\
1.112 \\
3.054 \\
2.05\end{array}$ \\
\hline 130 & 509 & $\begin{array}{r}1.164 \\
346 \\
0.679 \\
-\end{array}$ & $\begin{array}{r}1.224 \\
459 \\
0.902 \\
-\end{array}$ & $\begin{array}{r}1.292 \\
568 \\
1.117 \\
0.034\end{array}$ & $\begin{array}{r}1.416 \\
738 \\
1.449 \\
0.37\end{array}$ & $\begin{array}{l}1.728 \\
1.033 \\
2.030 \\
3.21\end{array}$ \\
\hline 160 & 680 & $\begin{array}{r}1.093 \\
325 \\
0.477 \\
-\end{array}$ & $\begin{array}{r}1.148 \\
430 \\
0.633 \\
-\end{array}$ & $\begin{array}{r}1.210 \\
532 \\
0.783 \\
-\end{array}$ & $\begin{array}{r}1.324 \\
690 \\
1.014 \\
0.016\end{array}$ & $\begin{array}{r}1.607 \\
961 \\
1.413 \\
1.70\end{array}$ \\
\hline
\end{tabular}

\footnotetext{
* Key $\quad 1.315$ Ratio of concentration by flashing.

390 Final concentration of $\mathrm{SiO}_{2}$ (ppm).

1.594 Final saturation ratio over solid AS.

0.010 Molecular deposition rate in micrometers/day at $\mathrm{pH}=7: 0$ and $\left[\mathrm{Na}^{+}\right]=0.069$.
}

Deposition rates calculated using kinetic expressions given in Chapter III of this report.

One stage flashing has been assumed for simplicity. 
CHAPTER FIVE - THE STATUS OF GEOTHERMAL BRINE TREATMENT TECHNOLOGY

Overview

The purpose of this Chapter is to give an overview of the practical experience and state of the art in geothermal brine chemistry and brine treatment technology. The emphasis is on defining what sorts of brine treatments may be needed by a typical geothermal electric development project, what is avallable, and how the developers may expect to proceed in dealing with anticipated brine chemistry related problems.

The author's own work in brine chemistry has been on the problems of preinjection silica removal, and that is reflected here. Much of the work in brine chemistry is proprietary or, if not proprietary, is still under way and has not yet been published. The discussion of such work is necessarily limited by the author's limited knowledge of it and by applicable standards of professional confidentiality.

\section{S5.1 The nature of the problem}

Undisturbed brines in a geothermal reservoir are in chemical equilibrium with the solid phases they are in contact with. The large temperature drops and steam losses brines undergo during the processes of brine production and energy extraction may cause various sollds to precipitate from them.. The Initial characteristics of the brine and nature and operating parameters of these processes determine what precipitates, in what quantity, and where. The dependence on Initial conditions and process parameters is strong and not very well understood. It is often the case that experiments with brine specimens from different wells in the same field or with rather similar synthet lc brines give strikingly and inexplicably different results. Extrapolation from fleld to fleld is more hazardous st11, even if the brine compositions and initial temperatures are similar.

In most cases, the major precipltate 1s either calcium carbonate or colloidal amorphous silica. Massive carbonate precipitation is more typical of moderate temperature brines (the classic example is East Mesa), and 
massive amorphous silica precipitation is limited to hotter brines (Niland, Cerro Prieto). The total amount of precipitate may range up to several hundred grams of solid per metric tonne of brine in the case of silica. Smaller amounts of other phases such as amorphous iron silicate, iron sulfide, lead sulfide, or iron carbonate are also frequently observed. In general, the importance of these "exotic" phases increases with increasing brine salinity and initial temperature. Except for the most extreme cases (i.e., Niland) they usually amount to only a few grams per tonne of brine. As a rule, they are recognized as being a problem in and of themselves only when not overwhelmed by either calcium carbonate or silica precipitation. Even at Niland, where the amount of iron silicate and the various sulfides approaches the amount of silica, they do not present a separate problem simply because they precipitate along with the silica. Calcium carbonate or strontium or barium sulfate may also precipitate out when "incompatible" brines are mixed.

At present, quantitative predictions can be made only in regard to the precipitation of calcium carbonate and amorphous silica from low and moderate salinity brines, and even this predictive capability is not complete or very reliable. It is possible to calculate the solubility of either under the given chemical conditions, and to calculate the rates of nucleation of colloidal silica particles and their growth. It is not yet possible to predict the kinetics of formation of solid carbonate or silica scales or the behavior of suspended particles. The theoretical and numerical methods involved in making these predictions are so complicated that this capability is now only beginning to be avaliable outside of the National Laboratories. In regard to the other phases, the existing predictive capabilities hardly extend beyond the vague generalities above.

The practical significance of a chemical change that takes place in the brine also varies with local conditions and is equally hard to predict. For purposes of discussion, we divide the general category of "practical impacts of scaling and solids precipitation" into two subcategories:

1) impacts of scaling on the production wells and energy conversion system

2) damage to reinjection wells. 
In general, binary cycle power plants are much more vulnerable to scailing problems because of the extreme sensitivity of their thermodynamic and economic performance to "fouling" of the heat exchange surfaces. The maximum allowable scale bulldup on the heat exchange surfaces before a cleanout is mandatory is on the order of a few tenths of a millimeter. Flash cycle plant systems are much less vulnerable; cleaning becomes necessary only when enough scale builds up to interfere with brine flow or valve operation. This means that, in most cases, only calcium carbonate and amorphous silica scaling have practical significance in a flash steam system; other kinds of scale simply do not bulld up rapldly enough to cause problems in and of themselves. On the other hand, even trace amounts of the "exotic" phases may be enough to render a binary plant project uneconomical. Fortunately, binary cycle systems are less likely to encounter the very high scaling rates sometimes observed in flash steam systems." Both types of system are equally vulnerable to producing well damage caused by rapid scaling at the producing horizon or horizons. To the author's knowledge, it is not yet possible to predict the rate of scale formation for any kind of geothermal scale well enough to quantitatively predict the economic impacts of scaling for any given site or power plant design.

Damage to the reinjection wellbore may be caused either by plugging with solids that are suspended in the brine being injected, or by precipitation of solids from the brine after it has been reinjected. In situ precipitation may be caused by slow reactions that did not go to completion in the surface equipment (such as amorphous silica polymerization), by the reheating of the brine (for example, the solubility of calclum carbonate decreases with increasing temperature), or by mixing with "Incompatible" reservior brine (for example, a calcium-rich injected brine mixing with a bicarbonate-rich reservolr brine). In situ precipitation is basically the same as scaling, and presents the same problems of prediction. The concentration of suspended solids in the brine may be easily measured in the field, and an analogous problem of prediction is absent.

However, even if prediction or measurement of the quantity of solids proves possible, 1t is usually not known exactly where these solids will form and/or accumulate downhole, or what their practical effect on injection well 
performance will be. At present, this may be meaningfully predicted only in the simplest cases, for example, suspended solids that accumulate at the sandface without significant invasion of the pore space to give a "filter cake" with well characterized properties.

Detalled prediction of the effects of any given brine treatment are equally hard to make; e.g., everyone knows and can predict that acidifying the brine can prevent the precipitation of calclum carbonate and slow down that of silica, but very little else can be predicted even this well. The variation of the effect of any given brine treatment from well to well and field to field is at least as great as is the variation in the chemical behavior of untreated brines. The practical effect of this is that one cannot simply design a brine treatment system from general principles.

The net practical result of all this is that progress in understanding and mastering the chemical behavior of the brine in any given area relies heavily on extensive field tests. In general, the following may need to be evaluated by means of detailed field testing in any given area before full scale development may proceed:

1) The location, nature, and quantity of solids precipitated from the unmodified brine. Ideally, this should include careful chemical and petrographic analysis, and a detailed theoretical interpretation which includes consideration of the chemical changes that the flowing brine itself undergoes. A state-of-the-art interpretation is worth having, because it will maximize the probability of making a valid and useful extrapolation to commercial conditions which are, as a rule, somewhat different from test or pilof conditions.

2) If carbonate precipitation is a major problem, it is necessary to determine the cause in detail (i.e., flashing downhole or mixing of incompatible fluids), and, if needed to prevent precipitation, what inhibitors or acids to inject into the brine, in what amount, and where.

3) The injectability of the brine in the chemical state in which it will be reinjected must be evaluated. At a minimum, this should include determination of the amount and nature of any suspended solids by membrane filtration (Barkman and Davidson, 1972), and core flushing experiments to evaluate the ability of the brine to plug the reservolr rock. If at all possible, these tests should be done in conjunction with brine treatment pilot plant 
experiments so that the brine employed realistically represents that which a commercial scale brine treatment facility at that site will deliver to the reinjection well. The core specimens employed should closely resemble the actual reservoir rock or, better yet, be prepared from samples of it. A detalled theoretical study of the chemistry of the brine to be reinjected should be included in order to assess the probability of such things as precipitation of solids caused by reheating post-reinjection or mixing with (potentially) incompatible fluids native to the injection horizon.

Unfortunately, the state of the art is such that even the above course of studies may not be able to give a definitive answer regarding injectabi11ty. At most sites, the development and evaluation of brine reinjection technology goes through the stage of a large scale injection experiment that involves one or a few wells. To be definitive, this large scale test must duplicate full scale brine treatment and reinjection facllity operating conditions as closely as possible.

4) If a preinjection brine treatment process proves necessary, this process must be evaluated on the pllot plant scale at the given site. Once again, the conditions of the pilot experiment should resemble full-scale operating conditions as closely as possible.

Part or all of the above course of studles has been executed or is in progress at a number of geothermal areas. These Include: Ähuachapán (EI Salvador), Broadlands (New Zealand), Cerro Prieto (Mexico), East Mesa, Heber, Jemez Caldera, Niland (aII in the U.S.), Otake (Japan), and Walrake1 (New Zealand). Commercial power generation 1s under way at Ahuachapán and otake with reinjection, and at Wairakel and Cerro Prieto without reinjection. Commercial power generation with reinjection at East Mesa, Niland, and Jemez Caldera is in the advanced planning stage.

Because the material in this Chapter is of a generic rather than sitespecific nature, it would have been somewhat clumsy to scatter references to publications dealing with specific areas throughout the text. Instead, it was decided to summarize all needed references to the literature in this one place. For further detalled information about any given fleld, consult the following sources:

Ahuachapán: Cuéllar (1975) and Elnarrson, Vides and Cuéllar (1975). 
Geothermal brine treatment in general: Phillips, Mathur and Doebler (1977).

Broadlands and Wairake1: Rothbaum and Anderton (1975)

Cerro Prieto: Weres, Tsao, and Iglesias (1980)

Niland and the general subject of injectability testing as applied in geothermal practice: Aust1n, et al. (1977), Netherton and Owen (1978), Piwinski and Netherton (1977), Owen, et al. (1977), and Quong, et al. (1978).

Otake: Kubota and Aosaki (1975), and Yanagase, Suginohara and Yanagase (1970).

\section{S5.2 Brine treatment technology}

The cumulative experience at and related to these various resource areas has led to the recognition of and development of a number of brine treatment concepts and component subprocesses. This includes a number of brine production and energy conversion system design strategies intended to eliminate or reduce the quantity of precipitated solids. All together, these might well be termed the unit processes that are available to the chemist or chemical engineer charged with developing a brine treatment process for a given area. In Figure 5.1, the range of available unit processes is graphically presented as the schematic for a hypothetical brine treatment system which contains all of them. (This is not actually very far from the truth; some of the brine treatment systems presently under consideration contain most of them!) Most of the unit processes and components depicted have to do with removing colloidal amorphous silica from flashed brine to make it fit for reinjection; this accurately reflects the historical emphasis of work in applied brine chemistry.

The headings in the following discussion refer to the labels in Figure 5.1. . 1. The brine enters the producing wellbore from the reservoir matrix. A. The producing wellbore. If the brine in question precipitates calcium carbonate when flashed, flashing in the wellbore should be prevented if at all possible. If the well is self-flowing, this is best done by reducing 
the brine: flow rate and/or increasing the diameter of the well if this is practical in the given case. Downhole pumping is an alternative which allows greater flow rates without wellbore flashing. However, downhole pumps have not yet been demonstrated in commercial service. Also, if the problem is. actually being caused by flashing in the formation near the wellbore, downhole pumping alone will not help.

Severe wellbore scaling may also be caused by the mixing of incompatible brines from different producing intervals within the wellbore. This problem may be identified by downhole sampling of brine at various depths or by careful interpretation of data on the thickness of scale and brine flow rate as a function of depth. It can be easily eliminated by completing wells in such a way that all brines entering any given well are compatible. 2. Addition of acid or scale inhibitors to the brine. The use of commercial scale inhibitors and hydrochloric acid to prevent carbonate scaling in surface equipment has bren successfully demonstrated at East Mesa. These chemicals may also be added to the brine at the bottom of the production well by pumping them down a small diameter tube placed in the wellbore.

The cost of chemical addition for carbonate scale control depends on the amount of chenlicals needed, and this depends on local conditions. The cost may, therefore, be anything from negligible to prohibitive.

The addition of such chemicals may also cause undesirable side effects In some cases. For example, carbonate scale Inhibitors may combine with calcium ion in the brine to form so called pseudoscale which may be as serious as the original carbonate scaling problem. Also, it is not known what happens when brine with scale inhibitors in it is reinjected. One possibility is that the irhibitor will be removed from the brine by adsorption on rock surfaces. If the brine is still supersaturated with calcium carbonate at this point, precipltation will occur and may damage the injection well.

Addition of acid prevents carbonate precipitation by actually preventing supersaturation with calclum carbonate. Lowering the $\mathrm{pH}$ also inhibits the precipitation of amorphous sillca by slowing down the rates of polymerization and coagulation. To effectively inhibit the polymerization of silica (which is usualiy considered to be the primary effect) it is necessary to reduce the $\mathrm{pH}$ to about 3. This usually requires an uneconomically large amount of acid 
and can cause serious corrosion problems. Also, a brine with this much acid in it will dissolve carbonate minerals in the reservior matrix after it is reinjected, and may cause serious and hard to repair damage to the injection we11. This sort of drastic acid treatment cannot be considered practical. The $\mathrm{pH}$ change needed to prevent carbonate precipitation or to inhibit silica coagulation is much smaller, and, in most cases, would not cause corrosion problems or injection well damage. However, the inhibition of silica coagulation is undestrable if colloidal silica is to be removed from the brine at some later stage of processing. In this case, it can be considered to be an undesirable side effect of carbonate control.

No "surface type" inhibitors are presently avallable for silica. (Commercially avallable carbonate and sulfate inhibitors are mostly of this kind.)

Hydrochloric acid and inhibitors may also be added to the brine at other points in the system.

B. The energy conversion process may be tallored to reduce or eliminate precipitation. The benefits of a binary system in this regard have already been discussed. With a flash steam system, a careful choice of separator pressure may also help control precipitation. For example, at Ahuachapán the brine is flashed down to about $150^{\circ} \mathrm{C}$ and reinjected at that temperature and the corresponding pressure. Because these conditions correspond to equilibrium between amorphous silica and dissolved silica for the amount of silica that is present in the brine, this completely eliminates silica precipitation problems which would otherwise be severe. Of course, such detailed process design is very site specific, and requires a good understanding of the chemical properties of the brine in question if success is to be assured. For example, reinfecting the flashed brine at Cerro Prieto at $150^{\circ} \mathrm{C}$ with no other brine treatment would probably cause severe silica scaling in the reinjection well simply because there is considerably more silica in the brine at Cerro Prieto than is the case at Ahuachapán.

C. Brine aging for silica scaling control has been tested at Ahauchapán, Broadlands, Otake and Wairakel, and 1s presently being tested at Cerro Prieto. The purpose of the aging step is to allow the dissolved silica to 
polymerize to colloldal form. This greatly reduces silica supersaturation and, thereby, the rate at which dissolved silica deposits on surfaces. This stops the cementation process which converts electrostatically coagulated colloidal silica to solid scale.

The rate of the polymerization process varies enormously with local conditions. At Cerro Prieto, the reaction runs its course in a few minutes while at Wairakei, it requires two hours. (This is one of the few things in geothermal brine chemistry that can be meaningfully predicted by theoretical and/or laboratory simulation methods.)

The practical effect of brine aging also varies with local conditions. At Ahuachapán and Otake, brine aging greatly decreased the rate of scale deposition and caused the scale that was deposited to be much softer and much easier to remove. At Broadlands, the scale deposited by the aged brine was softer, but the rate of scale deposition was unchanged.

Brine aging also has the effect of increasing the particle size of the colloldal silica. This is desirable if the silica is to be removed because larger silica particles usually coagulate better.

The remaining operations in Figure 5.1 are the unit operations available for preinjection brine treatment. The central operation of preinjection brine treatment is the removal of colloidal solids (mostly amorphous silica) from the brine by means of coagulation. Clearly, this requires that the colloidal silica coagulate. This usually happens spontaneously or is easy to Induce in medium and high salinity brines like those at Niland and Cerro Prieto. As will be discussed below, coagulation is much harder to induce in low salinity brines and no really satisfactory method is avallable to remove silica in this case.

Al1 equipment in a preinjection brine treatment system should be designed to minimize heat loss and to keep air out of the brine. Good temperature control is usually easy to achieve in a commerclal sized facility because of the large size of the mass flows and equipment. Keeping air out may be harder but is very important in order to prevent corrosion and the precipitation of substances like ferric hydroxide.

3. Coagulants may be added to the brine to cause or accelerate silica coagulation and settling. The cholce and concentration of coagulant is extremely site specific. For example, anionic polymeric coagulants help at 


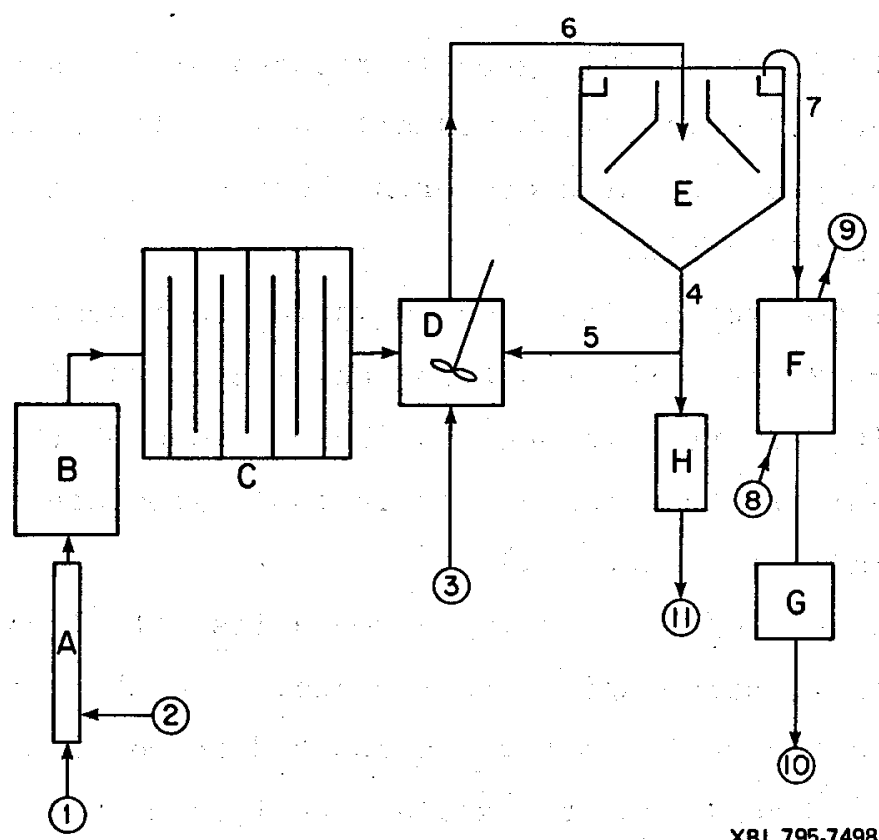

Fig. 5.1. Schematic of a hypothetical geothermal brine treatme that includes all possible unit processes.

Niland, but have no effect at all at Cerro Prieto. At Cerro Prieto, some (not all!) cationic polymeric coagulants give very good results with some wells, but not with others. At both Cerro Prieto and Niland, increasing the brine $\mathrm{pH}$ by about 0.5 unit by adding either lye or 1 ime works well. At Cerro Prieto, the amount of lime needed is small enough for lime addition to be economically attractive, while the much more strongly buffered brine at Niland would require so much that the process might not be practical there for that reason alone.

With high salinity brines like those at Cerro Prieto and Niland, the main purpose of the added lime is to coagulate the sllica by increasing brine $\mathrm{pH}$ rather than to actually react with 1 t. With very low salinity brines like those at Wairakei and Broadlands, coagulation is much harder to induce, and the lime removes the silica from the brine by actually reacting with it to produce a precipitate of amorphous calcium silicate. This sort of treatment requires much larger amounts of lime, and can cause a calcium silicate scaling problem. It can only be considered a treatment of last resort.

Increasing brine $\mathrm{pH}$ always carries with it the possibility of causing or increasing the precipitation of calcium carbonate. However, this may not 
actually present a practical problem because, under these circumstances, the calclum carbonate may be expected to precipitate out with the coagulated silica. In the case of brines which are buffered mostly by silicic acid (which is the case at Cerro Prleto, but not at Niland), Increasing the pH also has the desirable effect of increasing the solubility of silica and, thereby, reducing or eliminating silica supersaturation without actually having to remove dissolved silica from solution.

D. The coagulant(s) may be added to the brine and the coagulation reaction carried out in a separate stirred reactor, or the mixing unit may be made an integral part of the clarifier (unit $E$ ). Both configurations are equally effective. The author considers a separate mixer to be preferable for pilot plant work because it offers maximum process flexibility and ease of monitoring.

6. The brine goes from the stirred reactor to the clarifier.

E. Most of the coagulated silica is removed from the brine in the clarifier. Clarifiers are routinely used in municipal water treatment and sewage treatment applications, and are readily available from commercial suppliers. A reactor-clarifier which combines the functions of the reactor and the clarifier in one unit may be preferable for full scale application because it simplifies the overall brine processing system.

The single most important parameter in the design of a brine treatment system is the clarifier upflow rate; $1 . e .$, the rate of brine flow through the clarifler divided by the area of the clarifier. This quantity can only be determined by means of pilot testing.

4. The coagulated silica leaves the clarifier in the form of a dilute sludge which consists mostly of water but, nonetheless, contafns most of the coagulated silica and represents only a small fraction of the fluid flow through the clarifier.

H. If the sludge is to be disposed of as solid waste, it must be concentrated and dewatered in order to convert it into a relatively dry and compact solid material. The equipment needed for this is readily avallable but can contribute substantially to the overall cost of the process. In a few applications dewatering might not be necessary. For example, at Cerro Prieto the untreated sludge will simply be dumped in the existing brine evaporation pond. 
11. The sludge, whether dewatered or not, must be disposed of properly because it usually contains some toxic metals. With American regulatory standards, this will usually mean burial of dewatered sludge in a lined pit. 5. Part of the sludge may be recirculated; 1.e., put back into the brine at the point that the coagulant is added to 1 . The major effect of sludge recirculation is to rapidly decrease the concentration of dissolved silica in the brine to near saturation levels by supplying a large concentration of colloidal silica particles for the dissolved silica to react with. Recirculation is necessary at Niland because the rate of silica polymerization is rather slow there without $1 t$. The much more rapid silica polymerization rates observed at Cerro Prieto indicate that recirculation for this purpose may not be necessary there. Recirculation has the additional benefits of increasing the ultimate particle size and the rate of coagulation.

The optimal recirculation rate (if any) must also be determined by pilot testing.

7. The clarified brine leaving the clarifier may or may not need additional treatment prior to reinjection. As previously noted, the injectability criteria are highly site specific and must be determined by field testing. 8. Contacting the brine with either low pressure steam or condenser off-gas are possible additional operations. Steam would reheat the brine to $100^{\circ} \mathrm{C}$, and any oxygen that found its way into the brine during processing could be removed by steam stripping. (This might be desirable to reduce or eliminate corrosion in the reinjection system.)

Saturation with condenser off-gas, which consists mostly of carbon dioxide with smaller amounts of nitrogen and hydrogen sulfide (if untreated), would lower the $\mathrm{pH}$ to 6 or less. This final pH lowering would reduce or eliminate any residual supersaturation with calcium carbonate and slow down any chemical reactions involving silica that might still be going on. This final treatment might be particularly desirable if the silica removal process involved increasing the $\mathrm{pH}$, in that it would prevent the possibility of calclum carbonate or calcium silicate precipitation postreinjection. On the other hand, it would undo the silica solubility increase brought about by previously increasing the $\mathrm{pH}$. Also, condenser off-gas is almost always contaminated with air (grossly so if a contact condenser is used), and contacting the brine with it would inevitably introduce some oxygen. 
At Niland, it was found that contacting the brine with untreated condenser off-gas removed most of the hydrogen sulfide from the latter. The chemical basis of this process is the reaction of hydrogen sulfide with the abundant transition and heavy metal ions in that brine. Because most brines contain much less of these elements, the applicability of this process appears to be limited to Niland and, possibly, other nearby very high salinity reserviors. Reaction with condenser off-gas for this purpose would probably be placed in the process prior to sillca removal so that the precipitated metal sulfides would precipitate out with the silica.

F. Standard equipment is available for contacting the brine with steam or off-gas, but could prove expensive because of the large volume of brine involved. Also, the degree of $\mathrm{pH}$ lowering attainable by contacting the brine with off-gas is fundamentally limited by the solubility of carbon dioxide in water, which is near 1ts minimum value in the temperature range of interest. Performing this operation under high pressure to overcome this limitation would almost certainly prove economically unfeasible.

9. If the brine were contacted with steam or hydrogen sulfide free off-gas, the excess gas could simply be vented to the atmosphere. If the gas to be vented contained hydrogen sulfide, it would probably have to be scrubbed before discharge.

G. Filtering the brine is a possible final step. The need for it depends completely on the concentration of suspended solids in the brine leaving the clarifier, and on the injectability criterla for the brine at the given site. For example, at Niland the clarifler overflow contains about $30 \mathrm{ppm}$ suspended silica, and the recelving formation is a tight sandstone. There final filtration is absolutely necessary in order to reduce the concentration of suspended stlica to an acceptable value of lppm or less. If clarifier performance were somewhat better or the formation permeability fracture rather than pore dominated, filtration might not have proved necessary.

\section{S5.3 outstanding research needs}

The field of geothermal brine chemistry needs research more than it does development (other than site specific pilot testing). The major difficulties in dealing with geothermal brines are that their chemical properties are 
poorly understood and that certain kinds of task specific additives are not avallable (1.e., silica polymerization inhibitors of the surface type and coagulants that are effective in low salinity media). All or most of the hardware needed for brine treatment is available and only needs to be tailored to the particular application.

Also not needed are additional "1iterature surveys", bibliographies, and paper studies of various kinds. This includes economic analyses (unless closely tied to site specific technical work) simply because the technology is not mature enough for them to be meaningful.

The author considers the following research work to be needed. Some of it is already under way, and its continuation is to be encouraged. 1) Wholistic interpretation of the chemical behavior of geothermal brines. This should center on the methodical application of the available brine $:$ equilibrium and kinetics modeliling codes to actual field data and to the results of laboratory brine simulation studies. It should also include the generation of additional fundamental results where needed.

2) The most pressing "fundamental need" is that for a successful and generally applicable method of calculating electrolyte solution properties at arbitrary compositions and temperatures.

3) A more modest need is to be able to better understand and predict the coagulation of colloidal amorphous silica. This capability would be immediately applicable in the area of preinjection brine treatment process design, and would expedite the development of coagulants suitable for the removal of silica from low salinity brines. Such a capability has already been attained in regard to the formation and growth of colloidal silica particles in solution, and attaining it in regard to the coagulation step should be no harder.

4) Study of the fundamental mechanisms of calcium carbonate precipitation. For example, the following basic questions still need to be answered: Does solution phase nucleation of carbonate particles play an important role? Is the precipitation of calcium carbonate rate limited? Will colloidal silica nucleate carbonate particles? Such questions have been answered for the case of colloidal silica, and the answers are already in use in brine treatment process design. 
CHAPTER SIX - DOCUMENTATION FOR COMPUTER CODE SILNUC

\section{S6.1 Introduction}

The computer code SILNUC numerically models the homogeneous nucleation and growth of colloidal particles of amorphous silica. It contains most of the results of our experimental and theoretical work, and is able to reproduce most of our experimental data to within experimental error.

The model of amorphous silica chemistry embodied in SILNUC consists of the various empirical and theoretically derived formulas presented in Chapter 3 of this report. It was fitted to experimental data gathered in "buffer only" and sodium chloride containing solutions over the temperature range 50 to $100^{\circ} \mathrm{C}$. We belleve that the results can be safely extrapolated at least up to $150^{\circ} \mathrm{C}$ biecause the data are good and the theoretical model with which they were fitted appears to be sound. Only limited kinetic data were generated above about $\mathrm{pH} 8$, and these data were, for the most part, not included in the overall data reduction process. Therefore, SILNUC should not be relied on very much in the high $\mathrm{pH}$ range even though it may in some cases produce accurate predictions even there.

The model embodied in SILNUC assumes that all dissolved silica is monomeric. This is consistent with our reduction of the experimental data presented in Chapter 3, and is not far from the truth over most of the range over which the model contalned in SILNUC was fitted and is valid.

Both the base catalyzed and fluoride catalyzed reaction mechanisms are included in SILNUC. Sodium chloride, sodium fluoride, and dissolved silica are the only solutes that are assumed to be present. However, as has been discussed in 53.14 , solutions that contain other dissolved salts may be well approximated by using the artifice of an "effective sodium chloride concentration" which is equal to the sum of the molar concentrations of chloride and bicarbonate at room temperature. After the "effective sodium chloride concentration" has been calculated, proceed with the problem as though sodium chloride at that molar concentration were the only salt present.

SILNUC models only the formation of collotdal particles of amorphous silica and their growth by further molecular deposition of dissolved silica upon them. Deposition of dissolved silica upon preexisting colloidal silica 
particles that are "added" to the brine at the beginning of the reaction can also be modeled. The temperature and $\mathrm{pH}$ may be specified as either constant or varying. Alternatively, the concentration of dissolved silica in ionic form may be specified, and SILNUC will calculate the $\mathrm{pH}$ at each point in time as the calculation progresses. This option allows the frequent case in which dissolved monosilicic acid is the dominant buffer to be treated by SILNUC alone without having to estimate or otherwise calculate the brine pH. Loss of water by flashing to steam may also be specified.

SILNUC does not model the flocculation or coagulation of colloidal silica, or its adhesion to solid surfaces and cementation to form solid deposits. Unfortunately, the data and theoretical models needed to quantitatively describe and predict these processes are not yet avallable.

The primary outputs calculated by SILNUC are the concentration of dissolved silica and number and size distribution of colloldal silica particles as a function of time. Various supplementary values such as the rate of molecular deposition on solid surfaces, the saturation ratio for dissolved silica and the surface tension of the silica-water Interface are also calculated. Perfect plug flow reactor conditions are assumed. In physical terms, this corresponds to a perfectly stirred reactor vessel that contains a "grab sample" of brine or to brine flowing down a pipe without backmixing.

SILNUC was developed using the MNF4 FORTRAN compiler and the CDC 7600 system at the Lawrence Berkeley Laboratory. With this compiler and computer, SILNUC requires $24 \mathrm{~K}$ words (octal) to execute, and a typical problem requires about 1 second of execution time. There is very little or no deviation from standard FORTRAN in the coding of SILNUC. It should run on most other CDC systems with any FORTRAN compiler with no modifications whatsoever, and should need only minor modifications to run on IBM or other systems.

This Chapter was written in enough detail to allow the serious reader to work up to modifying SILNUC if so desired. All but the first page of Section 6.2 , and all of Sections $6.3,6.4,6.5$, and 6.7 may well be skipped on the first reading.

A sample problem is presented and discussed in Section 6.8. A full listing of the code is presented in Appendix 6.1. Queries concerning SILNUC should be directed to: 
Oleh Weres

90-1140E

Lawrence Berkeley Laboratory

$415-486-5625$ or FTS $451-5625$

\section{S6.2 The Basic Algortthms in SILNUC}

SILNUC contains three major algorithms. The "molecular deposition" or particle growth algorithm calculates the rate of moleculer deposition of dissolved silica on existing colloidal silica particles and the particle... radil as functions of time. It also calculates the dissolved silica concentration as a function of time. The changes in the particle radil and dissolved silica concentration are calculated using the Runge-Kutta algorithm. The time-step for this calculation is controlled by comparing the fractional change in dissolved silica concentration and the mass-averaged fractional change in particle radi1 to preset control parameters.

Within SILNUC, the population of colloidal silica articles is described and dealt with in terms of discrete "classes" of particles. The particles within each class are assumed to all have the same radius. Each class is completely specified by the particle radius (contained in array CLRV and the number of particles in it (contained in array CLNV). There may be up to 300 classes present at any time. Thus, the number of particle radii that need be kept track of is equal to the number of particle classes that are present.

One or more classes with given radil and a given amount of silica in each may be specified to be present at the beginning of the calculation. This feature allows heterogeneous nucleation and the effect of "seeding": the brine with colloldal silica to be modeled.

The second major algorithm in SILNUC is the nucleation algorithm. Nucleation is modeled as the creation of new colloid classes. Ordinarily, new classes are created at constant time intervals of DELTH. The detailed procedure is as follows: the time of the last molecular deposition step, TCV(1), is compared to the time at which a colloid class was last nucleated, THL. If TCV(1).GE.THL+DELTH, the nucleation algorithm is executed, First, the dissolved silica concentration and temperature, $\mathrm{pH}$, etc., at the time THL+DELTH are determined by interpolation. Then the nucleation rate is calculated using the theoretical formalism described in 53.8 . The transient 
short time effects are approximately accounted for using Eqns. (3.8.4 and 5). The value of the "Collins time constant" used to evaluate the "correction factor" in Eqn. (3.8.4) is that calculated using Eqn. (3.8.5) at the time that the time step size for nucleation (DELTH) was last changed. (See Section 6.5). In an isothermal problem, the value of the time constant calculated at the beginning of the problem is used throughout.

A newly nucleated class of colloidal particles is then introduced. The number of particles in the class is taken to be equal to the nucleation rate as calculated above (called RANUCC in the code) multiplied by DELTH. The number of $\mathrm{SiO}_{2}$ units in each of the newly nucleated particles is taken to be $n=n^{*}+0.5 / Z$, where $n^{*}$ is the number of monomer units in the nucleus of critical size under the given conditions, and $Z$ is the Zeldovich factor. Thus, the newly nucleated particles are actually somewhat larger than the nucleus of critical size under the given conditions. This is consistent with the physical content of the formalism presented in $\mathrm{S3.8}$, in which the nucleation rate $I_{N}$ is actually the rate at which particles of the size $n^{*}+0.5 / 2$ are created. ' (See the detailed discussion of this in Feder et al., 1966, pp. 132-6.) Introducing newly nucleated particles at a size $n>n^{*}$ is also necessary in a mathematical sense in order to ensure that they continue to grow in the molecular deposition part of the calculation.

When a new class of particles "is nucleated," the number of classes (NCLC) is increased by one, and the concentration of dissolved silica is decreased by an amount equal to the mass of the nucleated particles so that the mass balance is preserved.

Particles may dissolve as well as grow under the proper conditions. If the radius of a class of particles decreases below a certain small value, that class is "completely dissolved." The number and radius of the particles in it are both set equal to zero, and the silica that had been contained in them is added to the dissolved silica concentration. However, this rarely happens.

Whether or not any classes of colloidal particles are specified to be present initially, a class consisting of one particle is "nucleated" at the start of the problem. Therefore, there is always at least one class of colloidal particles present. 
Throughout the calculation, the maximum nucleation rate that has been calculated up to the given time 1s remembered. Eventually, the nucleation rate will begin to drop rapidly as the concentration of dissolved silica is decreased by its converston to colloldal s1lica. When the calculated nucleation rate drops to below a certain preset fraction (RRMN) of the maximum recorded nucleation rate ( $R M M X)$, nucleation ceases. RRMN is preset to 0.001 . This serves to halt the proliferation of collold classes which contain an insignificant number of particles.

The third algorithm is the one that specifies the temperature, $\mathrm{pH}$, and fraction of water lost by flashing as functions of time. The temperature (array TEMPRV) and fraction of water lost by flashing to steam (array FLASHRV) are Input as a serles of discrete values at given time values. A series of $\mathrm{pH}$ values (array PHRV) may be specified the same way, but need not be. The base values of time (array TREFV) are common for all three. During the calculation the values of temperature, fraction of water flashed of $f$, and $p H$ are determined by linear interpolation between the input values. Only one value of temperature, flash fraction, and $\mathrm{pH}$ need be specified if a constant value is desired for each throughout the calculation.

Alternatively, $\mathrm{pH}$ may be calculated at each step from the ratio of silica In lonic form (1.e., $\mathrm{H}_{3} \mathrm{SiO}_{4}$ ) to total monomeric silica. Using this option Implicitly assumes that the brine is buffered mostly by monosilicic acid. This is usually the case if the brine is low in bicarbonate and has a $\mathrm{pH}$ above about 7 at $100^{\circ} \mathrm{C}$. It need not be the case if the $\mathrm{pH}$ is lower than 7 or if a substantial amount of bicarbonate $(e . g \cdot$, comparable to the amount of dissolved silica) is present. If the concentration of lonic silica is specified, the values of PHRV need not be specified, and will be ignored if they are.

The dissolved silica concentration at the time of a nucleation step is calculated by quadratic interpolation using the concentration values calculated in the last three molecular deposition steps (henceforth referred to as "MD steps"). These three concentration values are stored in array CSILV. The three corresponding values of the time are in array TCV. The three parameters of the fitting parabola are in array CSFV. CSFV is calculated from CSILV at each $\mathrm{AD}$ step using the matrix CFIM. CFIM is recalculated whenever the MD step size changes. After each nucleation step (henceforth referred to as "N step") the value of $\operatorname{CSILV}(1)$ (the most recently calculated 
element of (SILV) is corrected to account for the decrease in dissolved silica concentration that the newly formed particles represent, and CSFV is recalculated.

The algorithms which generate and control the printed output from SILNUC are discussed in $\mathrm{S} 6.4$.

\section{S6.3 Program Structure}

The program consists of nine subprograms. The main program SILNUC is devoted mostly to reading input cards and setting variables equal to default values. The program can be given several problems to run at one time. The Do loop that goes through the problems one by one is in SILNUC. A copy of every COMMON block used anywhere in the program is present in sILNUC for reference, even though most of them are not used there. All DATA statements are also in SILNUC. They are segregated by the kind of variables being preset. (Other variables are reset at the beginning of a new problem by executable statements in subroutines MASTER, SETUP and SPECIFY.)

SILNUC calls SETUP and MASTER. SETUP and MASTER are called only by SILNUC, and only once per problem.

SETUP decides at what physical time to start calculating the problem, calculates initial and maximum values for the $M D$ timestep, an initial value for the $N$ timestep, and similar quantities which control the generation of printed output. (See Section 6.5.) SETUP calls SPECIFY and SILKIN. (SPECIFY and SILKIN are also called by MASTER, DEPOSIT, and OUTPUT.)

MASTER controls the actual execution of each problem, and directly or indirectly calls all subprograms other than itself, SILNUC, and SETUP. MASTER contains the nucleation algorithm, decides whether to execute an MD step or an $N$ step next, changes the $N$ and $M D$ time steps whenever necessary, decides when to terminate nucleation and the problem itself, and periodically calls the subroutine OUTPUT.

MASTER calls SPECIFY, SILKIN, DEPOSIT, and OUTPUT. DEPOSIT and OUTPUT are called only by MASTER.

DEPOSIT executes each MD step. It calculates the change in dissolved silica concentration, together with SILKIN calculates the changes of particle radif, decides whether the change in dissolved sllica concentration andor 
the mass-averaged change in particle radil warrant a change in MD timestep, "completely dissolves" and removes colloid classes whenever necessary, generates printed output at the level of MD steps, calculates CSFV, and modifies CFIM whenever necessary.

DEPOSIT calls SPECIFY and SILKIN.

OUTPUT generates printed output which gives an essentially complete picture of the physical state of the problem at the time of the call. This includes the number and radius of the particles in each colloid class as well as their total mass.

OUTPUT calls SPECIFY and SILKIN.

The major function of SPECIFY is to determine the values of temperature (TEMP, In degrees $C$ ), the fraction of water that has been flashed off (FLASH), and the $\mathrm{pH}(\mathrm{PH})$ by linear interpolation between the arrays of input values. It also calculates various related quantities like the hydrogen ion activity (AH) and corrects the sodium chloride concentration (SMI), and the total concentration of fluoride present (TOTF) for the effect of water loss by flashing.

If the concentration of dissolved silica, sodium chloride and fluoride are specified in units of grams or moles per liter at room temperature at the beginning of the problem, SPECIFY converts these concentrations to units of grams or moles per kilogram of water. These units are used exclusively from then on.

SPECIFY calls no other subprograms.

SILKIN calculates most of the chemical parameters used in the program. These Include the solubility and saturation ratio of amorphous silica (SRR), the equilibrium constants for various acid-base equilibria, the various chemical rate constants and ionic activity coefficients, $\mathrm{pH}_{\mathrm{nom}}$ (PHN), the fraction of dissolved silica in tonic form (ALPSIL), the surface tension. (GAMMA), the Zeldovich factor (ZLD), the steady state nucleation rate under the given conditions (RANUC), and various related quantities.

SILKIN also calculates the rate of change of the particle radii of each collold class (array CLDV), and uses these values to calculate the particle radi1 (array CLRV). CLDV is dimensioned $1200=4 \times 300$ to provide room for the four values of the derivative function that the Runge-Kutta algorithm requires to be evaluated per time step. CLRV is dimensioned 600 to 
provide room to retain the values of the particle radil calculated at the end of the preceeding MD step. (These values are needed to determine the fractional change in particle radii in DEPOSIT.) The most recent and intermediate values of the particle radil are stored in the first half of CLRV. The value of the control parameter KRGC tells SILKIN which derivative evaluation cycle of the Runge-Kutta algorithm the calculation is on. KRGC=0 indicates that SILKIN has not been called by DEPOSIT during a MD step; rather, it has been called by SETUP, MASTER, or OUTPUT, and the nucleation rate and related values are to be calculated instead of the rates of change of the particle radil.

SILKIN contains a number of calculated IF statements which prevent the recalculation of quantities of which the values are certain not to change from the preceeding call.

SILKIN calls PHF and WATER, which are called only by SILKIN.

PHF calculates the values of the " $\mathrm{pH}$ functions" $\mathrm{F}\left(\mathrm{pH}, \mathrm{pH}_{\mathrm{nom}}\right)$ and $\mathrm{I}\left(\mathrm{pH}, \mathrm{pH}_{\mathrm{nom}}\right)$. (See $\$ 3.3$ and A3.1 for the defintions of these functions.) In the program they are represented by FPH and FINT, respectively.

PHF calls no subprograms.

WATER calculates the density (DENS) and dielectric constant (EPSD) of pure water, and the Debye-Huckel coefficients ( $\mathrm{ADH}$ and $\mathrm{BDH}$ ).

WATER calls no other subprograms.

\section{S6.4 Control of Printed Output}

The printed output of the main program SILNUC and that of SETUP are always generated. This output describes the input received and the values for the various control parameters that have been adopted for the given problem. Messages stating that either execution of the nucleation algorithm or the calculation as a whole has been ended for the given problem and the reason why are also always generated by MASTER.

Al1 other printed output is controlled by the value of parameter IPR, which has a default value of 3 .

If the input specifies IPR.LT.0, only the output described above will be generated. 
If the input specifies IPR.EQ.0, IPR will have the default value 3 or whatever value it had at the conclusion of the preceeding problem, if that value differed from 3.

Specifying values of IPR in the range 1 to 6 will cause the generation of output in addition to that described above. Each successive increase in the value of IPR causes the generation of output in addition to that generated by lower values.

The value of IPR may be changed during the calculation by using the control parameters IPR2 and TCP. When TCV(1).GE.TCP, IPR will be set equal to IPR2. The default values for IPR2 and TCP are 3 and $1 E 10$, respectively.

IPR.GE.1 causes the generation of a line of output by DEPOSIT at every one or few MD steps. How often this line of output is generated is determined by the control parameters $\mathrm{CDP}$ and KDP. The program remembers the number of the last MD step at which this output was printed (KLP), and the concentration of dissolved silica at the conclusion of that MD step (CLP). The line is next printed when the number of the MD step just completed NCND.GE.KDP+KLP, or when the concentration of dissolved silica at its conclusion CSILV(1).LE.CLP-CDP. (Elsewhere in the program the number of the last or current MD step is stored as KSTEP or KSTP. At all times, KSTEP=KSTP=NCND.) The default values of $C D P$ and $K D P$ are $0.001 \mathrm{~g} \mathrm{~kg}^{-1}$ and 10 , respectively. Setting $\mathrm{KDP}=1$ or $C D P=0$. will cause this line of output to be generated at every MD step. The default values are chosen to generate enough output so that "nothing is missed," but also to prevent the generation of output if only minute changes have occurred since the last line was printed. IPR.EQ.1 generates only enough output to follow the change of the most Important chemical parameters with time.

IPR.GE.2 causes two or three calls of the subroutine OUTPUT during the execution of the problem: at the beginning of the calculation just after MASTER has been entered, when nucleation is ended (1f it was specified to begin with) and at the end of the calculation.

IPR.GE. 3 causes the generation of a message whenever the MD timestep (DELTC) or the $\mathrm{N}$ timestep (DELTH) is changed.

IPR.GE. 4 causes additional calls of OUTPUT that are controlled by the parameters NDP and DTP. Whenever OUTPUT is called, it sets $\mathrm{KPN}=\mathrm{KSTEP}+\mathrm{NDP}$ and TPN=TSPEC+DTP. (TSPEC is the current value of the time. At the end of a 
MD step, $\operatorname{TCV}(1)=T S P E C$.$) If IPR.GE.4, OUTPUT will be called again whenever$ KSTEP.GE.KPN or TCV(1).GE.TPN.

IPR.EQ.5 or 6 is normally used only for debugging purposes, because the additional output generated when these values are specified is bulky and has little if any physical significance.

IPR.GE.5 causes a message to be generated whenever a colloid class is created by nucleation or completely dissolved.

IPR.GE.6 causes an additional line of output to be generated by DEPOSIT at every MD step. This line contains the arrays TCV,CSILV and CSFV (three elements each). Values of IPR.GT.6 have the same effect as IPR.EQ.6.

\section{S6.5 Control of Time Steps and the Start of Calculation}

The natural time scale for the calculation may vary over many orders of magnitude with the conditions specified. Therefore, control variables that have the dimensions of time cannot be directly preset or read in. Rather, they are indirectly specified, and then calculated by subroutine SETUP using an approximate measure of "the natural time scale" for the given problem.

A convenient measure of "the natural time scale": is the "induction time for nucleation," defined as the time that it takes for the Inttial concentration of dissolved silica to drop by $0.05 \mathrm{~g} \mathrm{~kg}^{-1}$. It is well established that "the induction time" varies approximately as the minus first power of the molecular deposition rate and the minus one-fourth power of the other factors in the expression for the nucleation rate (see $\mathrm{S} 2.18$ and $\mathrm{S3.14}$ ). We derived the following approximate formula for "induction time" thus defined:

$\operatorname{TAUN}(\min )=1.08 \mathrm{E}-6 \mathrm{R}_{\mathrm{md}}^{-1}\left[\mathrm{ZQ}_{\mathrm{LP}} \exp \left(-\Delta \mathrm{F}^{*} /\left(\mathrm{k}_{\mathrm{B}} \mathrm{T}\right)\right) \mathrm{r}^{* 2}\right]^{-1 / 4}$

(See Section 3.8 for definitions of the symbols.)

This value is an appropriate measure of the "natural time scale" when particles formed by homogeneous nucleation dominate the polymerization reaction. However, when colloidal silica is initially present, molecular deposi- 
tion on the preexisting particles may be more important than the formation and growth of new ones. In this case, the following quantity is a more appropriate measure of the "natural time scale":

$$
\text { TAUD }=\left(C-C_{0}\right) /\left(A_{c} R_{\text {md }}\right)
$$

where $C_{0}$ is the equilibrium solubility of silica under the given conditions and $A_{C}$ is the total surface area of colloidal silica intially present per kilogram of water.

If colloidal silica is initially present, SETUP evaluates both TAUN and TAUD, and then sets" the induction time" TAU equal to the smaller of the two. The timestep for nucleation is calculated as

$$
\mathrm{DELTH}=\mathrm{TAU} / \mathrm{DDH}
$$

where DDH is a control parameter that is preset to 64 . but may be changed by the input. This value of $\mathrm{DDH}$ typically results in the nucleation of about 100 collo1d classes before nucleation ceases.

The maximum time limit for the calculation is defined as

$$
\text { TCMA }=\text { TAU* TMAXM }
$$

and the time interval for calls to OUTPUT is calculated as

$$
\mathrm{DTP}=\mathrm{TAU} * \mathrm{DTPM}
$$

TIAXM is preset to 64. , but may also be read in. DTPM is always read in. To facilitate interpretation of the output, it is better that the MD timestep (DELTC) not have arbitrary values. We chose to require that it always be an integral power of two. SETUP calculates the initial value and the maximum permissible value of DELTC as 
HCMI $=$ RTAU/DHCMI
HCMA $=$ RTAU/DHCMA

where RTAU is the power of two, elther positive or negative, that is nearest (in a logarithmic sense) to TAU in value. DHCMI and DHCMA are control variables that are preset to 2048. and 4., respectively, but may also be read in.

At the beginning of the calculation DELTC is set equal to HCMI, and usually goes through several doublings in the first few $M D$ steps.

When temperature is constant $(e . g \cdot, N R E F=1)$, the algorithm described above is executed once and then control passes to MASTER which actually executes the calculation.

When temperature is specifled to vary, the program must decide on the proper time to start the calculation. In most cases of practical interest in which the temperature changes, it decreases monotonically. At the beginning, the brine may actually be undersaturated with amorphous silica. As the temperature drops and water is lost by flashing, the saturation ratio increases. In the range of values $1<S<3$, the value of TAU decreases very rapidly as time passes and $S$ increases. If the value of TAU is set equal to a value that was calculated when $S$ was not very far from untty but increasing rapidly, the time parameters calculated will be much too large to suit the interesting, later part of the problem.

To avoid prematurely initiating the calculation with time parameter values that are much too large, the code compares the value of TAU with another "measure of the natural time scale": the difference between successive elements of TREFV.

Specifically, the program first sets the "starting time" for the calculation, TSTART, equal to TREFV(1), and calculates the value of $S$ at that time. If $S<1$, it sets TSTART=TREFV(2), and so on, until, finally, it encounters $S>1$ at some time value TREFV(I). Then it calculates TAU and DELTH. If DELTH.LE. (TREFV(I+1)-TREFV(I)), 1t accepts these values of TSTART, TAU, and DELTH, and proceeds with the further calculations as above. If this condition is not met, it sets TSTART=TREFV $(I+1)$ and repeats the calculation, and so on, until it is met. Then control is passed to MASTER and the calculation proper begins. If this condition cannot be met, an appropriate message is printed and the problem is terminated. 
As the calculation progresses the value of: $S$ changes, and with it, "the natural time scale." If the temperature remains constant, $S$ will drop as dissolved silica is converted to colloidal silica. This case poses no problem. The value of DELTC gradually increases unt1l it is equal to HCMA, and nucleation ceases when the nucleation rate falls to below a certain value. If the temperature drops, the value of $S$ may continue to increase during the early part of the calculation. The value of DELTC presents no problem, because DELTC will continue being halved as many times as is necessary. In this case, provision must also be made to allow DELTH to decrease. Otherwise, too few colloid classes "would nucleate" to accurately model the actual, continuous course of the homogeneous nucleation process.

The value of DELTH is controlled by code near the beginning of MASTER. If homogeneous nucleation is still proceeding, a new, trial value of DELTH is calculated before proceeding to the next MD step. This value is called DHN. If DHN.GE.DELTH.AND.TCV(1).GE.(THL+DELTH), an $\mathrm{N}$ step is executed. If DHN.GE.DELTH.AND.TCV(1).LT. (THL+DELTH), and MD step is executed. In neither case above is the value of DELTH changed.

If DHN.LT.DELTH.AND.TCV(1).GE.THL+0.5* (DELTH+DHN), an N step is executed, and DELTH is set equal to DHN. If DHN.LT.DELTH.AND.TCV(1).LT.THL+0.5*(DELTH DHN), an MD step is executed, and the value of DELTH is not changed.

The decision to halve or double DELTC is made at the conclusion of each MD step by code in DEPOSIT and MASTER. Two numerical tests are used. The absolute value of the fractional change in the value of the concentration of dissolved silica over the last MD step is compared with the value of the control parameter EPSIL. If the change is greater than EPSIL, DELTC is halved. If the change is less than one-half of EPSIL, this is an indication that DELTC should be doubled, but is not sufflcient to double it by 1 tself. The other test consists of comparing the average of the absolute values of the fractional changes of the particle radil with the control parameter EPR. This average is weighted by the mass of silica in each colloid class. If the average is greater than EPR, DELTC is halved. If the average is less than one-half of EPR, this is an Indication that DELTC should be doubled. DELTC is halved whenever elther test indicates that it should be. It is doubled only when both tests indicate that it should be, and even then only if doubling it will not make it larger than HCMA. 
EPSIL and EPR are both preset to 0.02. In the simplest case of no added colloid, constant temperature, and no flashing, this value results in a fractional error in the conservation of mass for silica on the order of 1E-6. In the worst case of collold added, rapid temperature change, and large loss of water by flashing, this error is st 111 less than about $1 \mathrm{E}-3$. This error may be decreased by modifying the appropriate DATA statement in SILNUC to preset EPSIL and EPR to smaller values.

\section{S6.6 Input and Default Values}

SILNUC accepts only formatted input on punched cards. A minimal input deck that specifies one problem with no added colloid and constant values of temperature, etc., would consists of only four cards.

The first card specifies the number of problems in the deck:

Card 1: . NRUN

The remainder of the card sequence is then repeated NRUN times, once for each problem to be run:

Card 2: NREF, NCLC, INX, IPR, IPR2, KDP, NDP, ITPM, IMAX, IHCMA, IHCMI, IDH, IMAXM

Card 3: SILIN, SILOUT, PHI, SMLI, TOTFI, CSII, TCP, CDP

Next there follow NREF cards of the form

Card 4: $\quad \operatorname{TREFV}(K), \operatorname{FLASHRV}(K), \operatorname{TEMPRV}(K), \operatorname{PHRV}(K)$

$(4 \mathrm{~F} 10.6)$

and NCLC cards of the form

Card 5: $\quad \operatorname{CLRV}(\mathrm{K}), \operatorname{CLMV}(\mathrm{K})$

If NCLC is zero, cards of the type of Card 5 are not present.

Card 2 specifies the control parameters for the given problem: NREF is the number of elements in each of TREFV, TEMPRV, etc. NREF must be at least 1. 
NCLC is the number of colloid classes initially present. NCLC must be zero or a positive integer.

INX in the control variable that determines whether or not homogeneous nucleation occurs. INX=0 signifies no homogeneous nucleation. (NCLC must be at least one in this case.) INX=1 signifies that homogeneous nucleation is to take place.

NREF, NCLC and INX must always be specified, while the remaining variables on Card 2 need not be in the simplest case.

IPR determines the level of printed output generation (see Section 6.4). Leaving this fleld blank or putting 0 in it will cause IPR to remaln equal to the default value 3 or to the value it had during the preceeding problem. (Throughout this Section, "default value" is used in this "either or" sense. See the discussion at the end of this Section.)

IPR2 is the value to which IPR is set equal when TCV(1).GE.TCP. A blank field w11l cause IPR2 to remain equal to its default value 3 .

$\mathrm{KDP}$ is one of the control variables that determines the number of MD steps between lines of output generated by DEPOSIT. A blank field will cause KDP to remain equal to its default value 10.

NDP and DTPM are the variables that control the frequency of calls to OUTPUT if IPR.GE.4. A blank field causes NDP to remain equal to 1 ts default value 50. ITPM determines the value of DTPM:

\section{DTPM $=2 . * \star$ ITPM}

There is no default value for ITPM. However, this field is usually left blank, and this has the effect of specifying ITPM=0.

IMAX ts the maximum number of MD steps allowed. The default value is 1000. IHCMA determines DHCMA:

DHCMA $=2 . * *$ IHCMA

If this field is left blank, DHCMA will retain its default value 4. IHCMI determines DHCMI: 
If this field is left blank, DHCMI w111 retain its default value 2048. IDH determines DDH:

$\mathrm{DDH}=2 \cdot * * \mathrm{IDH}$

If this field is left blank, DDH is set equal to its default value 64. IMAXM determines TMAXM:

\section{TMAXM $=2 . * *$ IMAXM}

A blank fleld will cause TMAXM to retaln its default value 64.

Card 3 contains the chemical parameters which specify the initial conditions for the given problem and two additional control variables.

SILIN is the initial concentration of dissolved silica.

SILOUT is the dissolved silica concentration at which the calculation will be terminated.

The units assumed for SILIN and SILOUT are determined by the sign of SMLI. If SMLI.GT.O., the units of SILIN and SILOUT are taken to be $\mathrm{g} \mathrm{SiO}_{2}$ $\left(\mathrm{kg} \mathrm{H}_{2} \mathrm{O}\right)^{-1}$. If SMLI.LT.0., the units of SILIN and SILOUT are taken to be $\mathrm{g} \mathrm{SiO}_{2} \mathrm{~L}^{-1}$ at room temperature, and then converted to $\mathrm{g} \mathrm{SiO}_{2}\left(\mathrm{~kg} \mathrm{H}_{2} \mathrm{O}\right)^{-1}$ during the first call to SPECIFY.

In the simplest case, only SILIN and SILOUT need be specified on Card 3.

PHI is the value of the practical osmotic coefficient to be used during the calculation. If this field is blank, PHI will retain its default value 0.92 , which is good enough for most practical purposes.

SKILI is the concentration of $\mathrm{NaCl}$ that is initially present. If SMLI.GT.0., Its units are taken to be moles $\left(\mathrm{kg} \mathrm{H}_{2}\right)^{-1}$. If SMLI.LT.0., ABS (SMII) is taken to be the concentration of $\mathrm{NaCl}$ in units of moles $\mathrm{L}^{-1}$ at room temperature, and is converted to molal units during the first call to SPECIFY. If this field contains a zero or is left blank, SMLI will retain its default value 0.088 . (This value approximately corresponds to our "buffer only" experimental solutions. Use it only to compare your own calculated results with those presented in Chapter 3.)

TOTFI is the total concentration of fluoride initially present. If SMI.GT.0., TOTFI is taken to be in units of moles $\left(\mathrm{kg} \mathrm{H}_{2}\right)^{-1}$. If 
SMLI.LT.0., TOTFI is taken to be in units of moles $\mathrm{L}^{-1}$ at room temperature. CSII is the concentration of dissolved silica inftially present in Ionic form. The units assumed for CSII are determined by the sign of SMLI as with SILIN and SILOUT. If CSII.GT.0., the $\mathrm{pH}$ will be calculated from the ratio of Ionic silica to total dissolved silica at each step of the calculation and PHV need not be specified. If CSII.EQ.0, PHV determines the pH values and must be specified.

TCP is the time at which IPR is to be set equal to IPR2. A blank in this field w1ll cause TCP to retain its default value 1E10; in practice, this default value means that IPR will not be changed at all.

CDP is the second variable that controls the frequency of output lines generated by DEPOSIT. If this field is blank, CDP will retain its default value 0.001 .

$\operatorname{TREFV}(K)$ contains the sequence of time values that the temperature, etc., w11l be interpolated between.

FLASHRV $(K)$ is the fraction of the water that has been flashed off at time TREFV(K). It must be less than one. Negative values are allowed and signify dilution of the brine with pure water.

TEMPRV(K) is the temperature in ${ }^{\circ} \mathrm{C}$ at the time TREFV(K). Good results can be expected only if the temperature is always between about 40 and $150^{\circ} \mathrm{C}$. Values up to $250^{\circ} \mathrm{C}$ may be specified for purposes of approximate extrapolation. PHRV(K) is the $\mathrm{pH}$ at time TREFV(K). If CSII.GT.0., this field should be left blank. PHRV will be ignored if it is specified in this case.

CLRV(K) is the radius of the particles in the $K t h$ colloid class initially present in nanometers $(1 \mathrm{~nm}=1 \mathrm{~F}-7 \mathrm{~cm})$.

CLMV(K) is the amount of silica in the Kth colloid class. The units of $\operatorname{CLMV}(\mathrm{K})$ are either $\mathrm{g} \mathrm{siO} 2\left(\mathrm{~kg} \mathrm{H}_{2} \mathrm{O}\right)^{-1}$ or $\mathrm{g} \mathrm{SiO} \mathrm{L}^{-1}$ at room temperature as determined by the sign of SMLI.

The variables whose values are not specifled in the input will be set equal to the preset default values clted above only for the first problem in. the given job. For all problems beyond the first, the values that the various input variables had at the end of the preceeding problem will serve as the default values. For example, if IDH was specified as 7 for the first problem, and the corresponding field was left blank for the second problem, DDH will be taken to be equal to 128.0 when the second problem is executed. 


\section{S6.7 Limitations and Precautions}

What SILNUC does and does not model was discussed in S6.1. The major inaccuracy in the theoretical model that SILNUC embodies ts that it does not include the process of particle growth by fuston. (See the discussion in S3.12.) Therefore, as the concentration of dissolved silfca approaches the equilibrium level, the concentration vs. $10 \mathrm{~g}$ time curve calculated by SILNUC will "flatten out" prematurely and unphysically as in Figure 3.20. Fortunately, this incorrect behavior is easy to spot when the results are graphed up.

Also remember that with INX $=1$ and NCLC $=0$, SILNUC will proceed as though homogeneous nucleation were the dominant process even when given a case in which heterogeneous nucleation would usually dominate in fact. If the time scale of the calculation turns out to be suspiciously long, try "putting in" a few $\mathrm{mg} \mathrm{L}^{-1}$ of colloidal silica of size greater than the critical nucleus size under the given initial conditions. If this dramatically accelerates the drop off in dissolved silica concentration, you will have good grounds to suspect that heterogeneous nucleation will dominate in fact.

The algorithm in SILNUC also has some purely mathematical characteristics that could concelvably cause incorrect results to be generated under some circumstances. First, after the nucleation calculation has been terminated, it cannot be restarted. If the temperature and $\mathrm{pH}$ are constant or vary only slightly, this is no problem because under these conditions, the nucleation rate would go through an early maximum and then drop rapidly and monotonically with time. However, if the temperature drops rapid1y.and/or the $\mathrm{pH}$ increases rapidly, the rate of nucleation might go through a minimum and then increase again. The initial decrease is due to the depletion of dissolved silica by the growth of colloidal particles that formed early in the process. The subsequent Increase may be caused by a decrease in temperature, an increase in $\mathrm{pH}$, or both. A rapid decrease in temperature can cause the saturation ratio to increase despite a decreasing concentration of dissolved silica. An increase in $\mathrm{pH}$ will lower the surface tension. Note also that when the $\mathrm{pH}$ is controlled by MSA buffering, decreasing dissolved 
silica concentration and, even more so, decreasing temperature will cause the $\mathrm{pH}$ to increase. If SILNUC terminates the nucleation calculation during the period of falling nucleation rate, it will "miss" the possibly important "second crop" of colloidal particles formed when nucleation resumes.

This deficiency in SILNUC could be corrected by modifying SILNUC to allow nucleation to resume after it has been terminated, but we $d i d n^{\prime} t$ have the opportunity to do this. With the present form of SILNUC, all that one can do is to carefully examine the output for indications of trouble on this score so as not to be taken unawares. (See the sample problem in Section 6.8.) Fortunately, this problem is unlikely to arise in connection with what is probably the most important practical case: a very rapid temperature drop in the wellbore and steam separators, followed by essentially isothermal conditions in the brine treatment and reinjection systems. If the time scale of the initial temperature drop is shorter than the time scale of the silica polymerization process, nucleation does not terminate until after the initial rapid temperature drop is over, and the problem does not arise.

Another purely numerical problem can arise when one colloid class completely dominates the overall polymerization process; for example, this would happen if one were to try to model the course of one of our molecular deposition experiments using SILNUC (Section 3.4.) The problem arises because the expression we use to calculate the rate of molecular deposition on the surface of a colloidal particle is discontinuous at the value of $S$ at which the particle of a given radius $r$ is in equilibrium with the silica in solution. When the silica concentration drops to the value that corresponds to saturation with the dominant colloid class, this discontinuity will prevent the dissolved silica concentration and particle radius from setting down into their ultimate steady state values. Rather, they w1ll endlessly drift around in a quasiperiodic fashion in a small range of values that includes the actual steady state values. This behavior is easily recognized and easy to correct for: simply consider the steady state to have been reached at the point at which this begins to happen. of course, in nature particle growth by agglomeration and fusion would become the dominant growth process at this point anyhow. 


\section{S6.8 A Sample Calculation}

In this sample problem we model the chemistry of silica in the brine at Cerro Prieto. This is a relatively difficult problem that demonstrates most of the code's capabilities as well as some of its limitations. (Ca1culating isothermal homogeneous nucleation curves like those in Figures 3.18 , 3.20 and 3.24 to 3.28 is a relatively easy problem.)

The input deck for this problem is reproduced in Table 6.1, and the printed output is reproduced in Table 6.2 .

The first input card specifies that there is only one problem in this deck. The second card specifies that there are thirteen time base values between which the temperature and fraction of water lost by flashing are to be interpolated, that there are two colloid classes initially present, and that the nucleation calculation is to be performed. The third card spectfles the brine composition at the beginning of the problem. The initial total dissolved silica concentration is $0.597 \mathrm{~g}\left(\mathrm{~kg} \mathrm{H} \mathrm{H}_{2}\right)^{-1}$, of which $0.0075 \mathrm{~g}$ $\mathrm{kg}^{-1}$ is in Ionic form. The salts in the brine are represented by 0.18 moles $\mathrm{kg}^{-1}$ of $\mathrm{NaCl}$, and the initial total concentration of fluoride is 0.0006 moles $\mathrm{kg}^{-1}$. The calculation is to be terminated when the dissolved silica concentration drops below $0.25 \mathrm{~g} \mathrm{~kg}^{-1}$.

At time 0. , the temperature is $300^{\circ} \mathrm{C}$ and no water has been lost by flashing. (The $\mathrm{pH}$ is not specified because the concentration of silica in ionic form has been.) These initial conditions approximately correspond to saturation with quartz down in the reservoir.

Chemical equilibrium is destroyed when the brine begins to flash in or near the wellbore. This moment corresponds to time 0 . Starting at this time, the brine rapidly loses water by flashing and drops in temperature as 1t flows up the wellbore and through a three stage steam separator system. (The 0.5 minute intervals are meant to represent the residence time within the separators.) At 2.75 minutes it has 1 lost $41 \%$ of its water by flashing, and its temperature is down to $100^{\circ} \mathrm{C}$. It then flows through a pipe at approximately constant temperature for 10 minutes, and is dumped into an evaporation pond where it slowly cools down to ambient temperature. (This temperature sequence is meant to demonstrate some possibilities of practical interest rather than to describe what is actually happening at Cerro Prieto.) 
The last two cards specify the two classes of colloidal particles initially present. The particle radil are 3.5 and 7.0 nanometers $(=3.5 \mathrm{E}-7$ and $7.0 \mathrm{E}-7 \mathrm{~cm})$, and each class contains $0.005 \mathrm{~g} \mathrm{SiO}_{2}\left(\mathrm{~kg} \mathrm{H}_{2} \mathrm{O}\right)^{-1}$ at the start of the problem. These particles are meant to represent the small amount of particulate matter that is always present in natural brines. ( 0 course, in nature these heteronuclei would not consist of pure amorphous silica.)

In fact, the carbon dioxide that is inftially present in the brine in a concentration of nearly $1 \%$ by weight has a major effect on the $\mathrm{pH}$ while it is still in solution. However, most of tt is removed from the brine by steam stripping relatively early in the flaching process. By the time that the brine temperature has fallen to the point that silica polymerization begins, most of the carbon dioxide and other gases are gone, and MSA 18 the dominant buffer.

The sample output for this problem was generated with IPR set to its default value 3 .

The output begins with a printout of the input values and the various control parameters that were indirectly specified by the input and default values. In cases where default values have been substituted for blank fields or zeros in the input, the default values are printed out rather than what was actually input.

The first part of the output is that from the main program SILNUC. The four lines that begin with "KNUC=58" are from SETUP. " ANNUC is the number of monomer units in a particle of critical nucleus size. KNUC is ANNUC rounded off to the nearest integer value. AN 1s the number of monomer units In the particle actually "nucleated by the code" at the beginning of the calculation. ZLD is the Zeldovich factor.

$$
A N=\text { ANNUC }+0.5 / Z L D
$$

RLIM is the radius of a particle of size AN in centimeters. (Throughout - the printed output, all lengths are in rentimeters.) TAUN, TAUD, TAU and RTAU are as discussed Section 6.5.

The values in the fourth line may be used to estimate the effect of varying the value of the surface tension (GAMMA) on the Induction time for homogeneous nucleation (TAUN). (See Section 3.10.) All the output to this point is always generated, regardless of the value of IPR. 
The block of output between the two lines of $\neq$ signs is that generated by subroutine OUTPUT.

TSPEC is the time of the last call to SILKIN. In this particular case, it is the time at which the calculation was started. TEMP is the temperature in ${ }^{\circ} \mathrm{C}$. FLASH is the fraction of the water that has been flashed to steam. FLC is the factor by which the dissolved compounds and preexisting colloidal particles have been concentrated by flashing.

$$
\mathrm{FLC}=1 . /(1 .-\mathrm{FLASH})
$$

GLASH is the product of FLC and the time derivative of FLASH.

CSIL is the concentration of monomeric silica (both ionic silica and MSA) present in $\mathrm{g} \mathrm{kg}^{-1}$. (Throughout the printed output, all concentrations are given in terms of grams or moles per kilogram of water.)

"SOL.CORR.FACTOR" is the factor by which the solubility of AS is decreased by the presence of the sodium chloride.

PHN is $\mathrm{pH}_{\text {nom }}$ - FPH is $\mathrm{F}\left(\mathrm{pH}, \mathrm{pH}_{\mathrm{nom}}\right)$. FINT is $\mathrm{I}\left(\mathrm{pH}, \mathrm{pH}_{\mathrm{nom}}\right)$.

GAMZ is $\mathrm{H}_{\gamma}-\mathrm{TS}_{\gamma}$; i.e., it is what the value of $\gamma$ would be at the given temperature in the limit of negligible surface dissociation.

DQS is $n_{0}$, the number of ionizable sites per square centimeter on the surface of AS.

AKIN is $S_{t}$ (see "Eqns. 3.4.7). DKIN is an arbitrary "rate constant" assumed for the particle dissolution process. It is preset to unity. "TOT.F" is the total amount of fluoride present in molal units. . ALPF is the fraction of the total flouride that is present as $F^{-}$(rather than $\mathrm{HF}$ ).

RKF1 and RKF2 are $k_{H F}$ and $k_{F^{-}}$, respectively (see Eqns. 3.6 .6 and 7 ).

RATEKF $=$ TOTF* $($ RKF $1 *(1 .-A L P F)+R K F 2 * A L P F)$

RATEKT is $k_{\mathrm{OH}}(T)$ (see Eqns. 3.4.7e and $f$ ).

PKW, PKSIL and PKF are the values of $\mathrm{pK}_{a}$ for water, MSA, and $\mathrm{HF}$, respectively.

GSIL, GF and GNA are the single ion activity coefficients for $\mathrm{H}_{3} \mathrm{SiO}_{4}^{-}$, $\mathrm{F}^{-}$, and $\mathrm{Na}^{+}$, respectively. 
EPSD is the dielectric constant of pure water.

DENS is the specific gravity of pure water.

$\mathrm{ADH}$ and $\mathrm{BDH}$ are the two Debye-Hückel coefficients,

CSILS is the solubility of AS in pure water at the given temperature. ALPSIL is the fraction of the monomeric silica in lonic form.

CSI is the concentration of silica in lonic form in $\mathrm{g} \mathrm{kg}^{-1}$.

$S R A$ is $S_{a}$.

SRR is S.

RATEK=RATEKT* $($ FPH+RATEKF $)$

RDEPF is $R_{\text {md }}$ in $\mathrm{g} \mathrm{cm}^{-2} \mathrm{~min}^{-1}$.

FKINL is $r * 1 \mathrm{nS}$.

RNUC is the radius of the critical nucleus under the given conditions, and ANNUC is the number of monomer units that it contains.

$\mathrm{CN}$ is the forward rate of monomer deposition on the surface of a particle of critical nucleus size in $\mathrm{SHO}_{2}$ units $\mathrm{min}^{-1}$.

QT is the total partition function for the critical nucleus in units of $\left(\mathrm{kg} \mathrm{H} \mathrm{H}^{-1}\right.$. It $1 \mathrm{~s}$ equal to the hypothetical concentration of critical nuclei that would exist "at equilibrium" at the given value of $S$.

RANUC is the nucleation rate corrected for short time effects in units of $(\mathrm{kg} \mathrm{min})^{-1}$. (Note: In the code RANUC is used to represent the steady state nucleation rate, and RANUCC to represent the nucleation rate corrected for transient effects.)

TAUC is the "time constant" for the bulldup of the nucleation rate to its steady state value. TAUC is calculated using (3.8.5). The value of TAUC printed out here is that calculated for time TSPEC. NCLC is the number of colloid classes at the time OUTPUT is called. Even though the calculation has just started, NCLC has already been Increased to three because a new colloid class that consists of just one particle is created at the beginning of the calculation.

"TOTAL NO." is the total number of colloidal particles present per kilogram of water, and "TOTAL AREA" is their total surface area in $\mathrm{cm}^{2}$. "AVER.RAD." is the average radius of the colloidal particles in cm. The average surface area and mass are $1 n \mathrm{~cm}^{2}$ and grams, respectively. "COLL.MASS" is the amount of colloidal silica present in $\mathrm{g} \mathrm{kg}^{-1}$. 
"DIS.SIL." is the concentration of monomeric silica in $\mathrm{g} \mathrm{kg}^{-1}$. The total number and mass of the colloidal particles in each class are given in units of $\mathrm{kg}^{-1}$ and $\mathrm{g} \mathrm{kg}^{-1}$, respectively.

Most of the output on the following two pages consists of messages announcing that one of the time steps has been changed. In this part of the calculation, DELTH decreases at every step because the temperature is still dropping and the saturation ratio is increasing. If this were an isothermal problem, DELTH would not change.

TAUCS is the value of the "Collins time constant" calculated using Eqn. (3.8.5) at the same time that the value of DELTH was last changed. This value is stored and used to evaluate the "correction factor" in Eqn. (3.8.4) until it is next changed. TAUC in the output generated by OUTPUT is not the same as TAUCS; TAUC is calculated by evaluating Eqn. (3.8.5) whenever OUTPUT is called.

THN is the time of the last $N$ step, and $\operatorname{TCV}(1)$ is the time of the last MD step.

The time step change messages may be suppressed without effecting the rest of the output by specifying $I P R=2$.

Starting with KSTEP=22; the time steps cease to change, and the next 35 lines of output are from DEPOSIT. The column headings below the last DELTH change message label the line output from DEPOSIT.

"NUC.RATE" is the value of the nucleation rate, corrected for transient effects, calculated at the time of the last $N$ step. CAREA is the total surface area of the colloidal silica present in $\mathrm{cm}^{2}$. "NUC.FLUX" is the rate of conversion of dissolved silica to colloidal silica by nucleation of new colloidal particles in units of $\mathrm{g}(\mathrm{kg} \mathrm{min})^{-1}$.

"DEP.RATE" is the rate at which dissolved silica is converted to colloidal silica by molecular deposition on colloidal particles that already exist. It is not necessarily equal to the product of RDEPF and CAREA, because not all particles present are necessarily larger than the critical nucleus size.

OUTPUT is called again when nucleation is ended. Note that this happened only 0.32 minutes after the temperature reached $100^{\circ} \mathrm{C}$. The number of particles in each of the first three colloid classes and the sum of colloidal plus dissolved silica are slightly larger than at the first call of oUTPUT because of slight further water loss by flashing. 
In Iine output from DEPOSIT that was generated after nucleation ceased "NUC.RATE" and "NUC.FLUX" were set equal to zero because SILKIN calculates the nucleation rate only when called during a $N$ step or by OUTPUT. DEPOSIT prints out a newly calculated nucleation rate only once and then prints out zeros until a new value of the nucleation rate is calculated. Zeros can also occur in the DEPOSIT output before nucleation is terminated if the generation of line output is more frequent than are the $\mathrm{N}$ steps.

The temperature stays at $100^{\circ} \mathrm{C}$ until time $=12.75$ minutes. During the last four minutes at $100^{\circ} \mathrm{C}$ the dissolved silica concentration (CSIL) drops by only $0.012 \mathrm{~g} \mathrm{~kg}^{-1}$. This indicates that the calculation has almost reached a steady state even though $S>1.3$. This is caused by the deficiency in the model that is illustrated in Fig. 3.20 and discussed in Sections 3.12 and 6.8: there are very few particles that are large enough to keep growing by molecular deposition at this relatively low saturation ratio, and particle growth by particle fusion is not permitted. This is demonstrated by the fact that the area of the particles large enough to keep growing as estimated by dividing "DEP.RATE" by RDEPF is less than a tenth of CAREA at this point.

The calculation continues until the dissolved silica concentration drops below $0.225 \mathrm{~g} \mathrm{~kg}^{-1}$ at $41^{\circ} \mathrm{C}$. The results are only semiquantitative below about 70 or $80^{\circ} \mathrm{C}$ because of the rapid increase in $\mathrm{pH}$ and $\mathrm{pH}_{\mathrm{nom}}$ with decreasing temperature. One reason to distrust these results is because the values of $F\left(\mathrm{pH}_{1} \mathrm{pH}_{\mathrm{nom}}\right)$ and $\mathrm{I}\left(\mathrm{pH}, \mathrm{pH}_{\mathrm{nom}}\right)$ are extrapolated when the value of either argument is $>8$. More importantly, the value of the surface tension decreases rapidly and, in nature, this would cause nucleation to resume, but SILNUC is not capable of modeling this. That nucleation should have been restarted at lower temperature is obvious from the values of GAMMA, QT, and RANUC that are given in the output from the third and last call to OUTPUT. 
Table 6.1

Listing of Input Deck for SILNUC Sample Problem

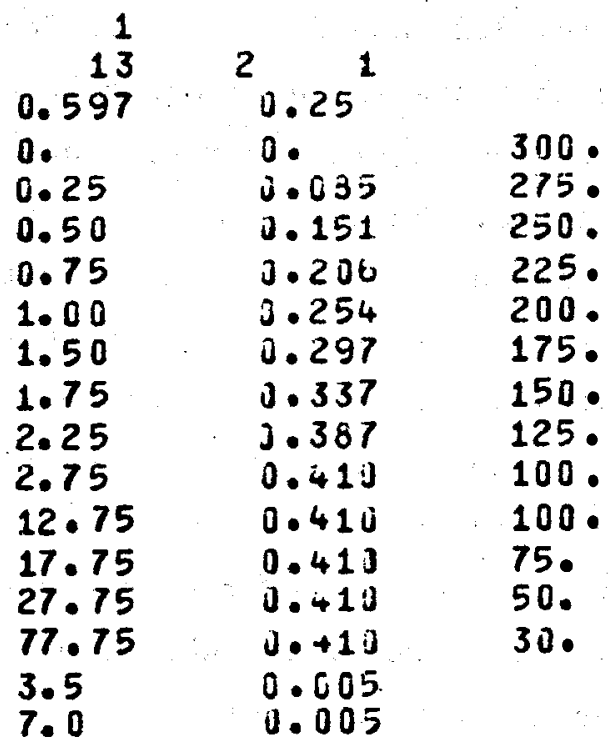

$0.18 \quad 0.0006 \quad 000075$

275 .

250 .

225.

200 .

175 .

150.

125.

100.

75.

50.

0.005

3.5

0.005 


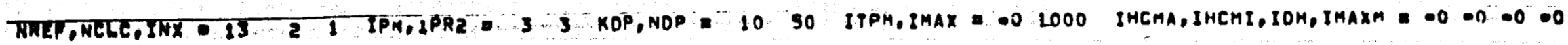

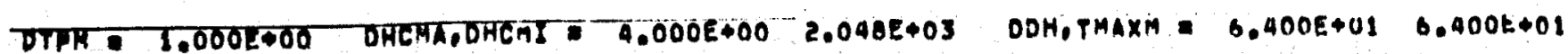

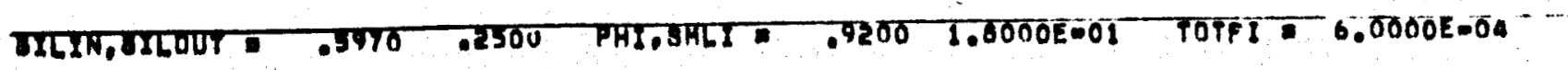

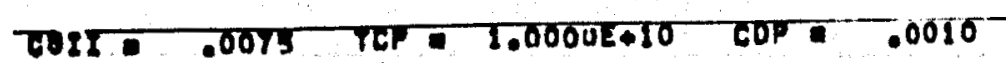

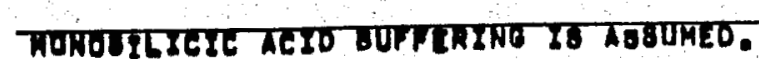

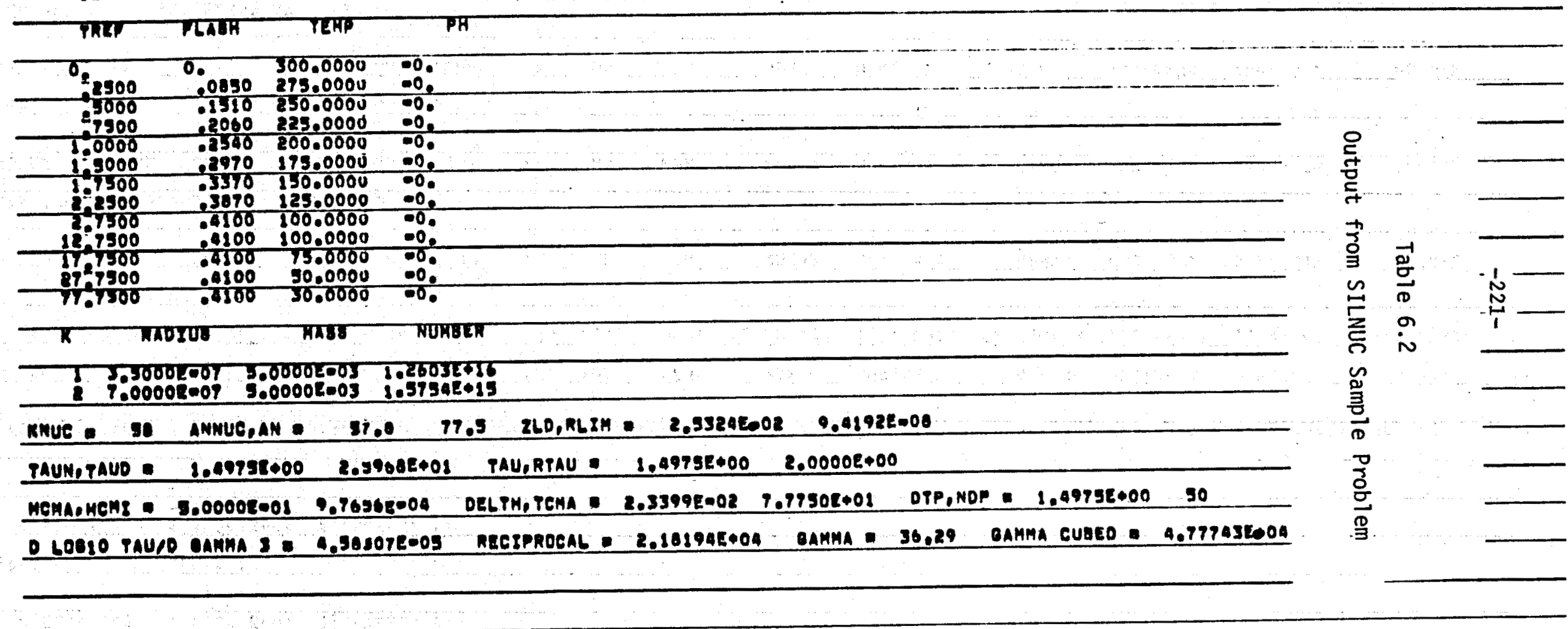

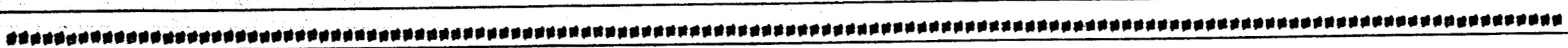

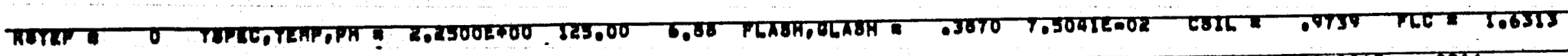

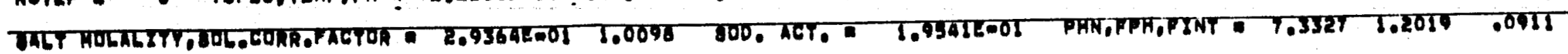

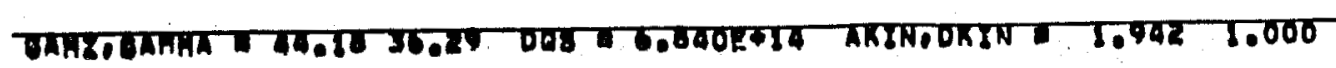

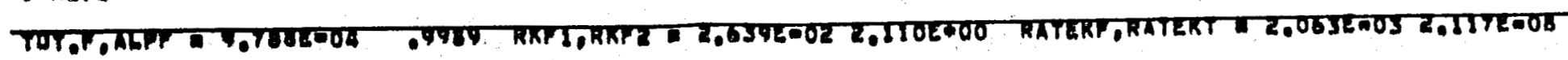

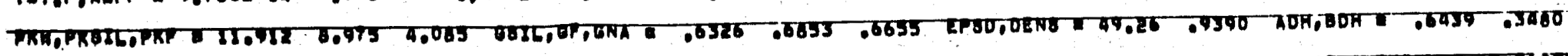

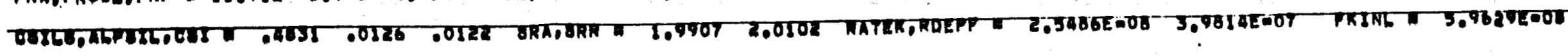




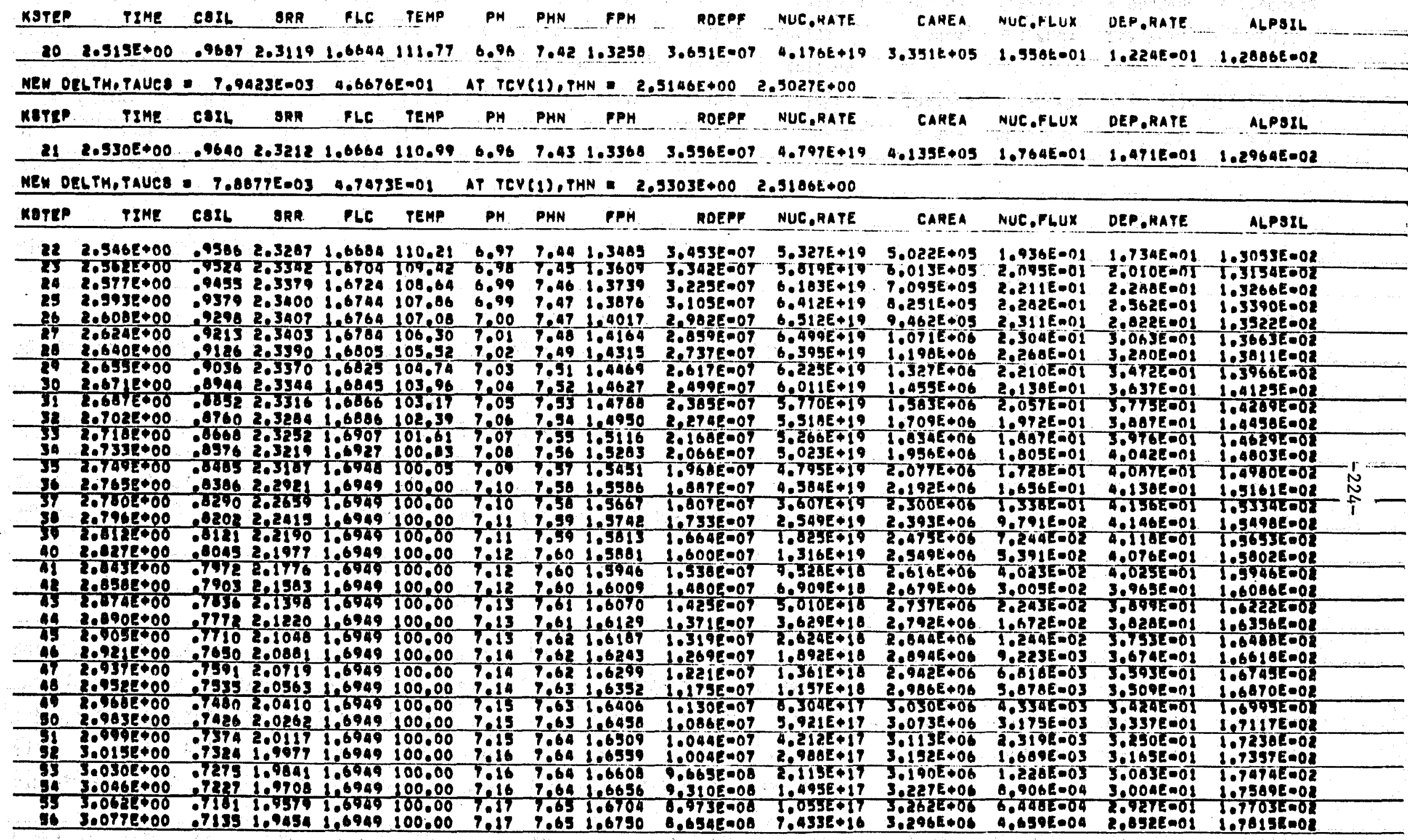

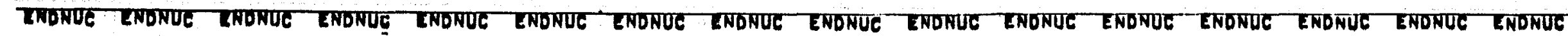

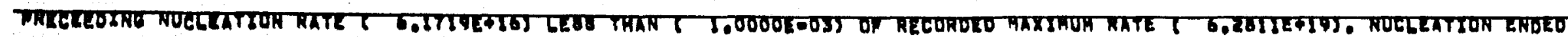




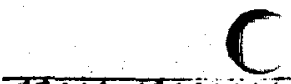

WOrep o ge

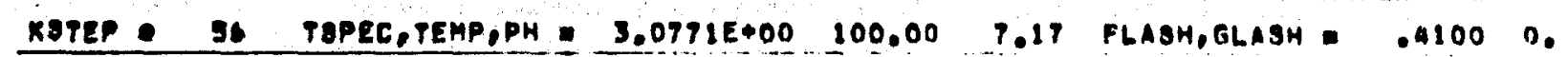

C816 - .7135 FLC $=1.6949$

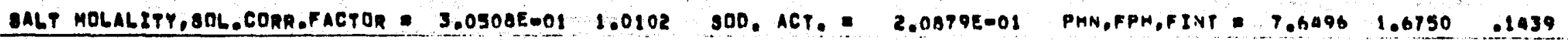

gAMZogAMMA 05.4033 .72 DOS $0.040 E+10$ AKINOOKIN 2.0001 .000

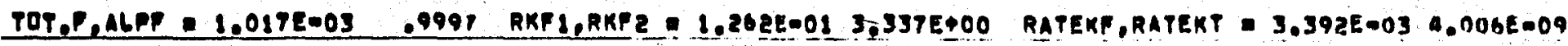

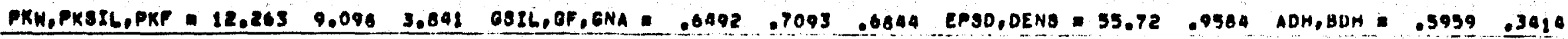

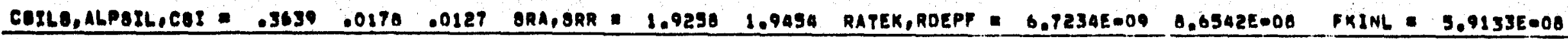

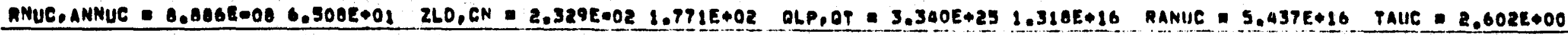

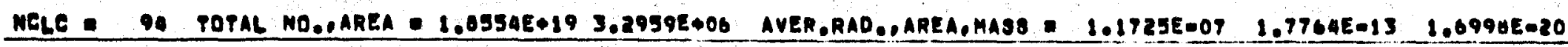

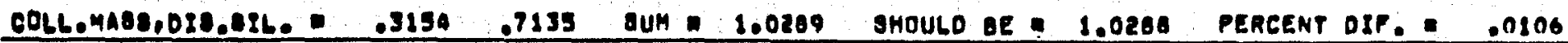

numaen of PARTzCLES IN EACH CLASB

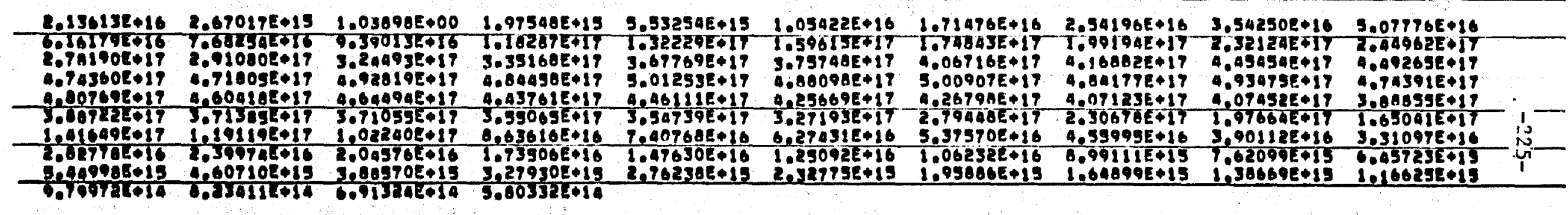

\section{Panrsele naors}

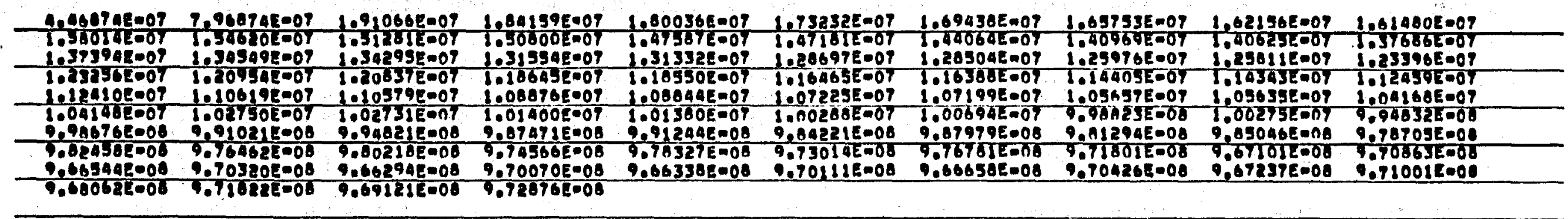

TOPAL MAOS IN EACH CLABS

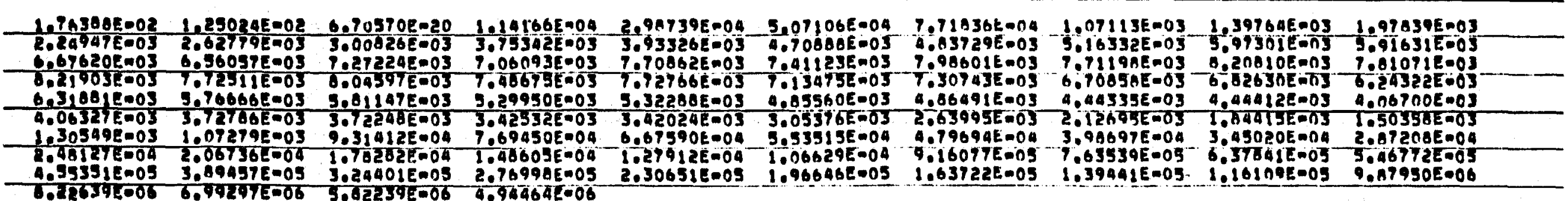

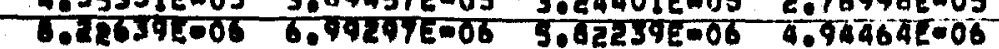




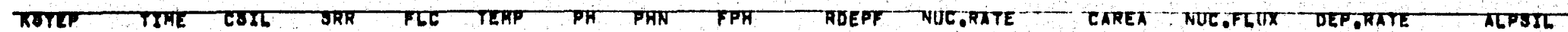

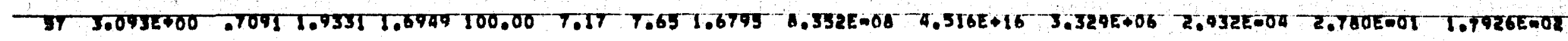
TLE VELTE - 3.T2BOLOER AT RSTEP : 57

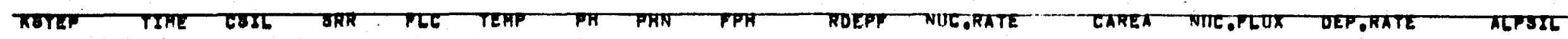

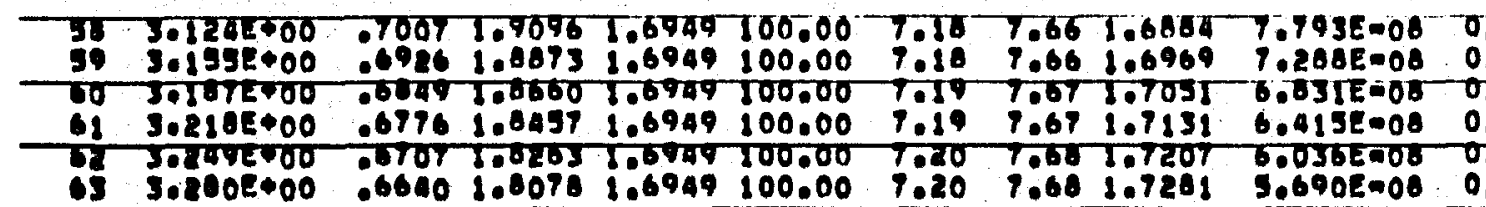

$3.392 E+06$

$-\frac{3.451 E+06}{3.307 E+106}$

$3.561 E+06$

ग.6

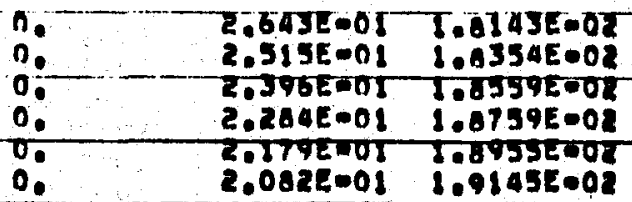

MEN OELTE -6.83902002 AT KBTEP 63

\begin{tabular}{|c|c|c|c|c|c|c|c|c|c|c|c|c|c|c|}
\hline neres & PRME & coil & SRR & ple & TEMP & PH & PHN & FPH & ROEPF & NUG.RATE & CAREA & IUC of LUX & EP.RARE & ALPSIL \\
\hline $\begin{array}{l}60 \\
89 \\
78 \\
71\end{array}$ & 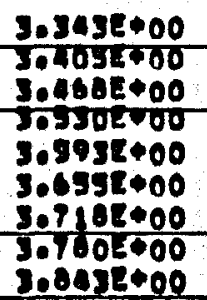 & $\begin{array}{l}.6519 \\
.6401 \\
.6296 \\
.0200 \\
.6112 \\
.6034 \\
.9969 \\
.9908 \\
.9049\end{array}$ & $\begin{array}{l}1.7123 \\
1.6056 \\
1.6612 \\
1.6397 \\
1.6203 \\
1.6029 \\
1.9670\end{array}$ & $\begin{array}{l}1.6949 \\
1.69149 \\
1.6949 \\
1.6919 \\
1.6949 \\
1.6919 \\
1.6949\end{array}$ & $\begin{array}{l}100.00 \\
100.00 \\
100.00 \\
100.00 \\
100.00 \\
100.00 \\
100.00 \\
100.00 \\
100.00\end{array}$ & $\begin{array}{l}9.21 \\
7.22 \\
7.22 \\
7.23 \\
7.24 \\
7.24 \\
7.25 \\
9.25 \\
7.26\end{array}$ & $\begin{array}{l}9.70 \\
9.71 \\
9.71 \\
9.72 \\
9.72 \\
9.73 \\
9.73 \\
9.79\end{array}$ & $\begin{array}{l}1.7423 \\
1.7535 \\
1.7679 \\
1.7795 \\
1.7903 \\
1.0000 \\
1.8068 \\
1.0160 \\
1.0043\end{array}$ & 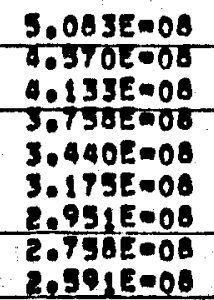 & $\begin{array}{l}0 . \\
0: \\
0 . \\
0: \\
0 . \\
0: \\
0 . \\
0 . \\
0 .\end{array}$ & $\begin{array}{l}3.747 E+06 \\
30.027 E+06 \\
3.900 E+06 \\
3.966 E+06 \\
4.026 E+06 \\
4.070 E+06 \\
0.124 E+06 \\
4.165 E+06 \\
4.202 E+06\end{array}$ & $\begin{array}{l}0: \\
0: \\
0: \\
0: \\
0: \\
0 .\end{array}$ & $\begin{array}{l}1.905 E-01 \\
1.74416001 \\
1.612 E=01 \\
1.400 E=01 \\
1.334 E=01 \\
1.130 E=01 \\
1.044 E=01 \\
9.475 E 002 \\
8.595 E=02\end{array}$ & $\begin{array}{l}1.9510 E-02 \\
1.98505002 \\
2.0589 E-02 \\
2.0504 E 02 \\
2.0799 E 002 \\
2.1067 E=02 \\
2.1313 E 002 \\
2.1590 E 002 \\
2.1791 E 002\end{array}$ \\
\hline
\end{tabular}

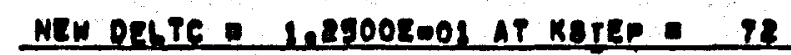

ROTEP TEME COIL ORR TLC TEM

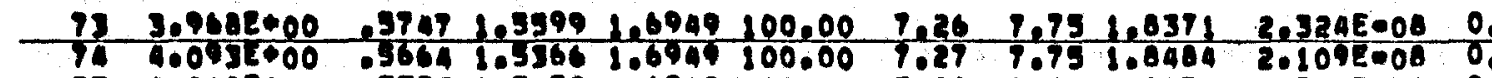

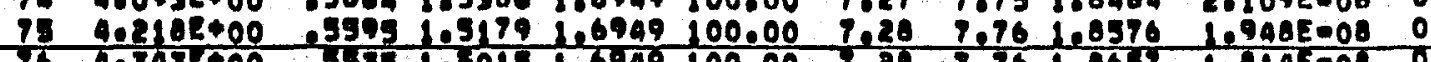

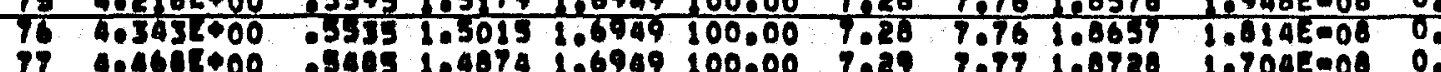

(19)

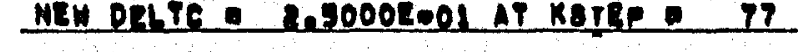

KOTEP TIME COIL IRR FLC TEMP PH PHN PPH ROEPF NUC,RATE CAREA NUE,FLUX OEP,RATE ALPSIL

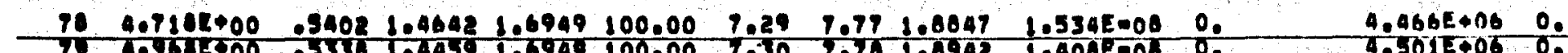

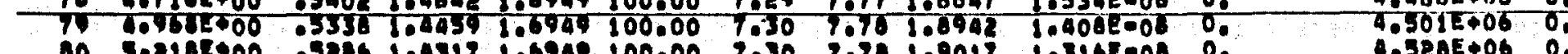

$803.2102+00 \quad 32861.4317 \quad 1.6940100 .00 \quad 7.30 \quad 7.701 .90171031620080$

MEW DFLTC B,0000E-01 AT KaIEP 00

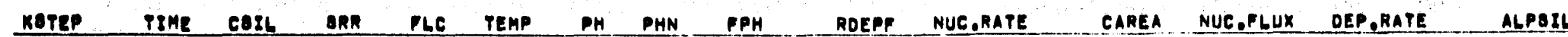

of $5.710 E+00 \quad 05200 \quad 1.4090 \quad 1.6949100 .00 \quad 9.31 \quad 9.791 .9130 \quad 8.102 E 0000$.

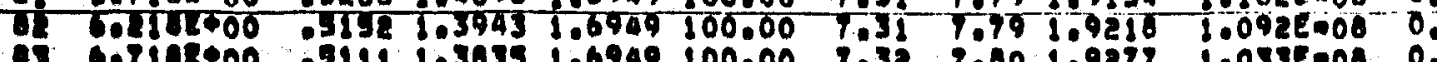

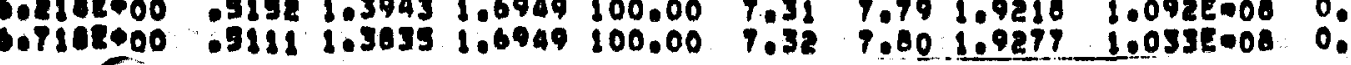

$4.366 E+06 \quad 0$
$4.391 E+06 \quad 0$

$4.6064+06$ O

$1.220 E-02 \quad 2.0418 E-02$

$6.5 n 7 E-03$ - 2.46036 .02

7.8706-03 2.0698R.02

$2.736 E-02 \quad 2.3532 E-02$ 2.1762002 2.03023L-61 


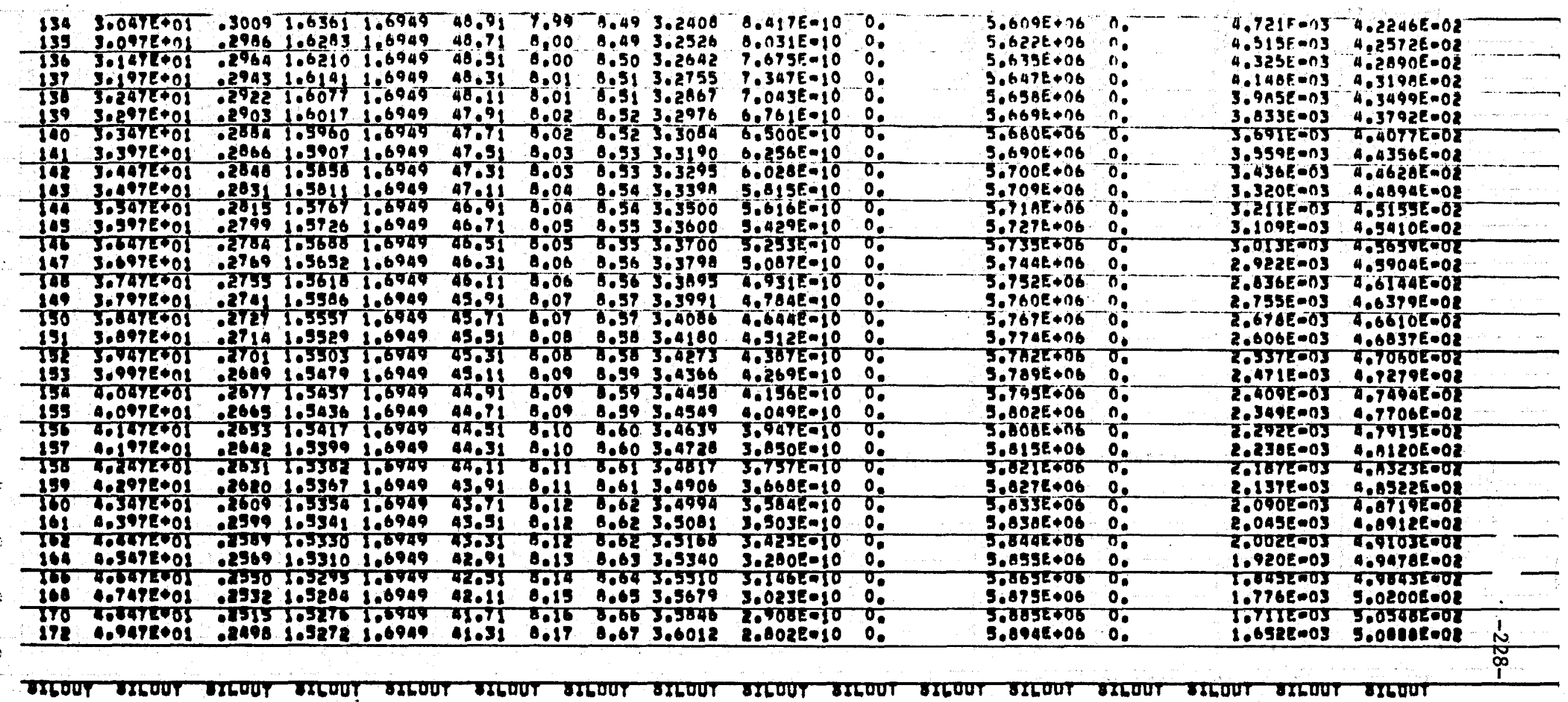

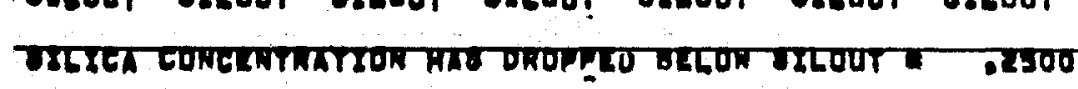

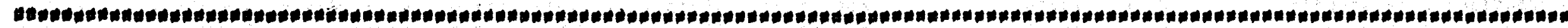

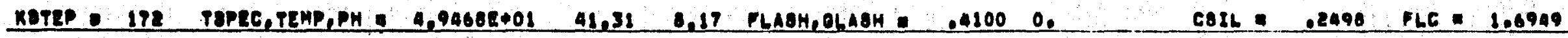

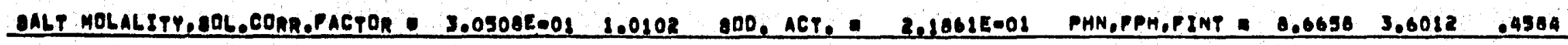

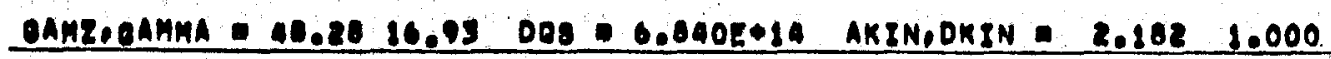

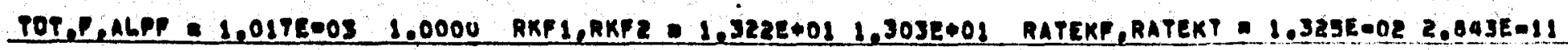

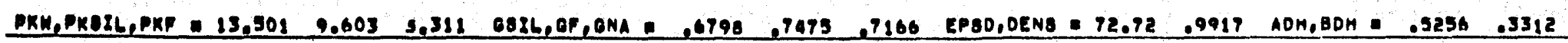

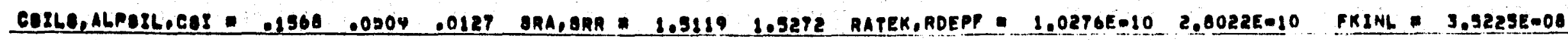

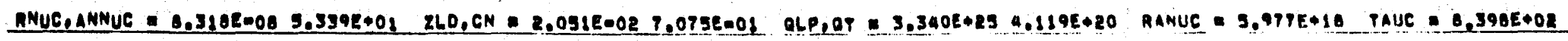

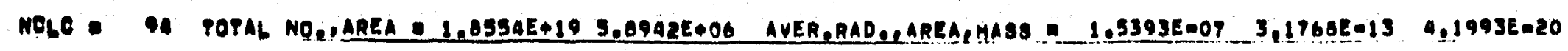

C. . . , , , , 


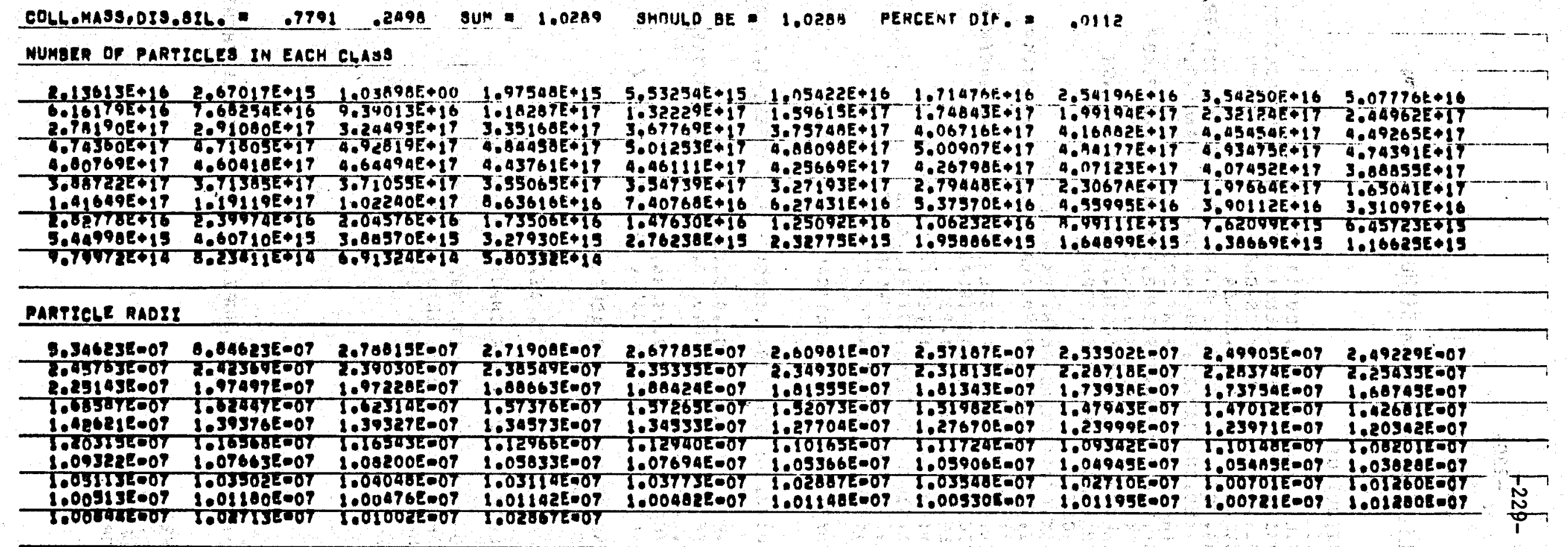

\section{TOYAL MAss IN EAEH CLABS}

\begin{tabular}{|c|c|c|c|c|c|c|c|c|c|}
\hline 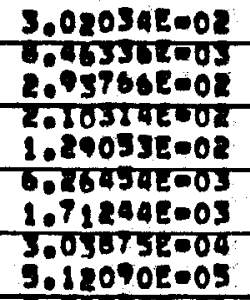 & 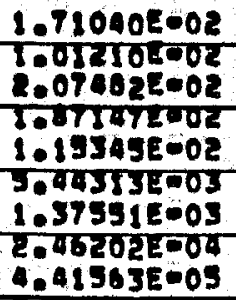 & 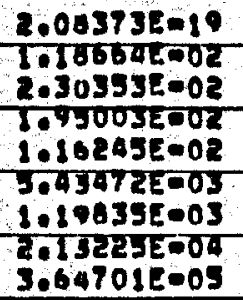 & 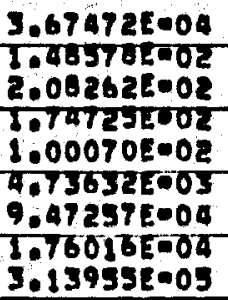 & 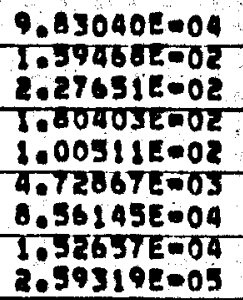 & 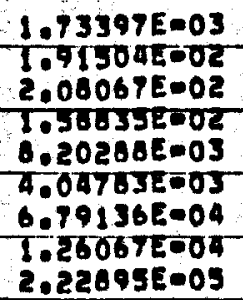 & 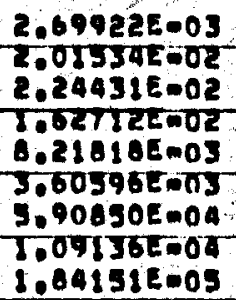 & 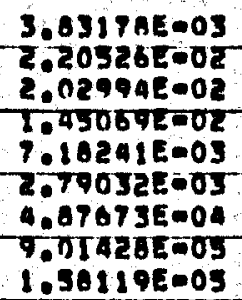 & 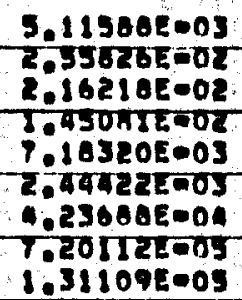 & 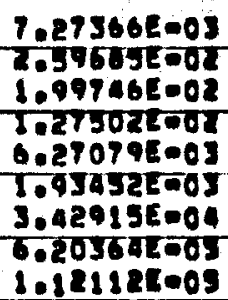 \\
\hline
\end{tabular}


Appendix 6.1

Full Listing of Program SILNUC

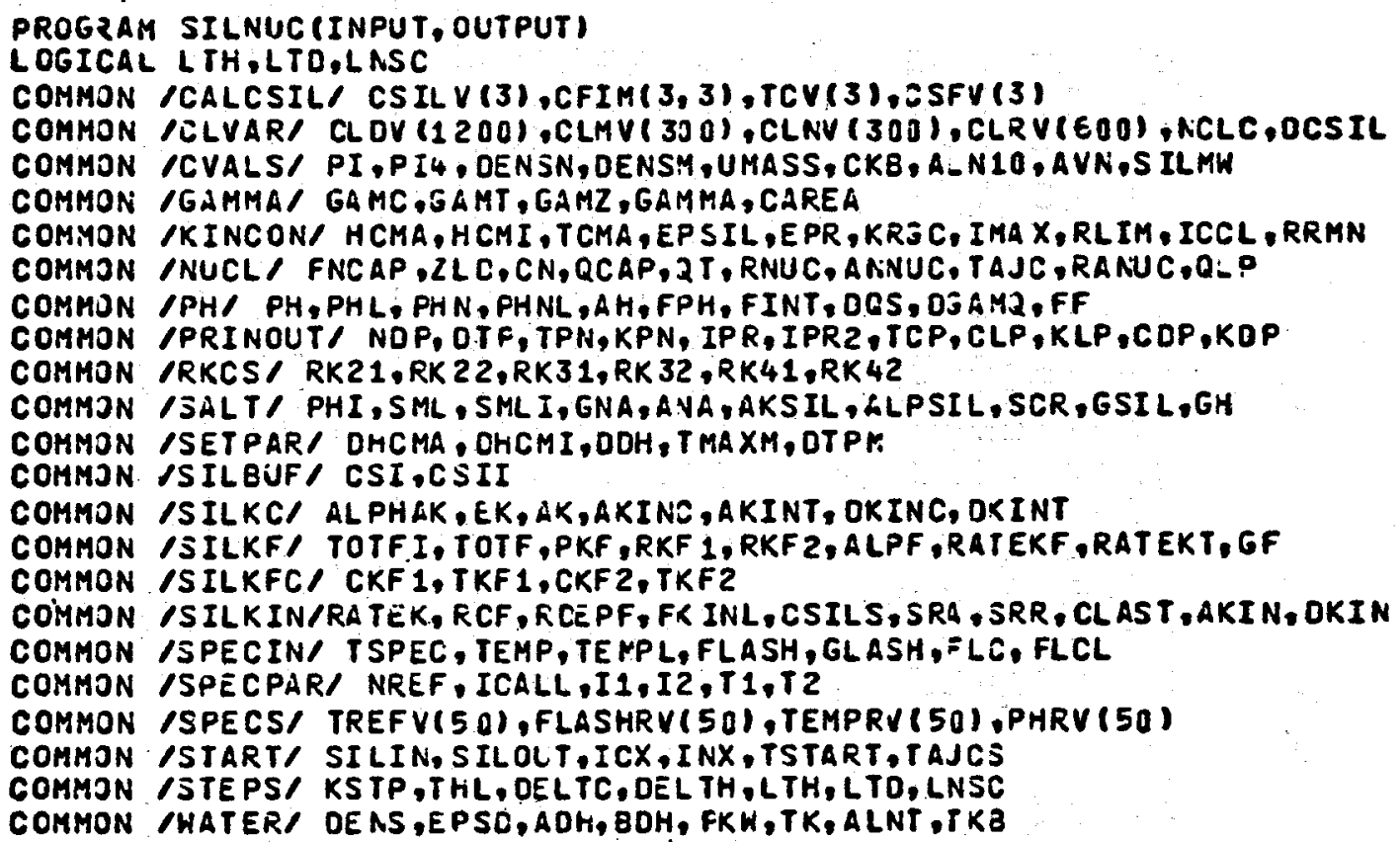

THE FOLLOHING ARE PHYSICAL AND MATHEMATICAL CONSTANTS: LEAVE THEM ALONE.

DATA PI,CKB,AVN,SILMW/3.14159265359,1.38054E-16,5.02252E23,60.085/

DATA YMOLE/2T.2I

DATA RK21,RK22,RK31,RK 32,RK41,RK42100,0.5,0,.1,.2,.2.1

DATA GAMC,GAMT, OQS,FF, QLP/50.30, $-0.049,6.34=14,0.45,3.34 E 251$

DATA ALPHAK,EK,AK/5.,4296.6,3.1171/

DATA AKINC, AKINT, CKINC, OKINT $10.0977,75.84,0.00 .1$

DATA CKF1,TKF1,CKF2,TKF2, $-11.723,4039 .,-2.647,1183 .$,

$\mathrm{C}$
$\mathrm{C}$
$\mathrm{C}$
$\mathrm{C}$
$\mathrm{C}$
$\mathrm{C}$
$\mathrm{C}$

c

THE FOLLOHING ARE PRESET CONTROL IARIABLES.

CHANSE THEM IF YOU. DARE.

DATA EPSIL,EPR, RRMN $/ 0.02,0.02,0.001 /$

THE FOLLOWING ARE DEFAULT VALUES FOR INPUT VARIABLES.

DATA IPR, IPR2,KDP, NOP, IMAX $/ 3,3,10,50,1000$,

DATA PHI,SMLI,TCP,COP / $0 . \$ 2,0.088,1 E 10,0.001 /$

DATA DHCMA, DHCMI, DOH, TMAXM / 4., $2048 ., 64 ., 64$, ,

UHASS $=$ S ILMH/ AVN

DENSN=AVN/VMOLE

DENSN= DENSN*UMASS

$P I 4=\$ * P I$

ALN1J = ALOG 116.$)$

READ 1, NRUN

DO 5 KRUN=1, NRUN

REAO 1, NREF, NCLC, INX, IPRI, IPR I2,KDPI, NOP I, IT PM, IMAXI, IH CMA, IHCMI,

1. IDH, IMAXM

$I C X=1$

$I F(I P R I \bullet N E \cap O) \quad I P R=I P R I$

IF(IPRI2.NE Q Q) IPR2 =IPRIZ

$I F(K O P I \bullet G T \bullet O)$ KDP $=K D P I$

IF (NDPI GT.O) NDP $=$ NDPI 
OTPM $=2, * * I T P M$

IF (IMAXI.GT.O) IMAX =IMAXI

IF $(I+C M A . G T \cdot 0)$ DHCM $=2$ *** I HCMA

IFIIHCMI.GT:O) DHCMII $=2 .+$ IHCMI

IF $(I O H . G T . O) \quad O D H=2 . * * I C H$

IF (IMAXM, GT, O) TMAXM=2,**IMAXM

PRINT $12, N R E F, N C L C$, INX,IPR, IPR2,KOP,NDP, ITPM, IMAX, IHCMA, IHCMI, IDH,

1 IMAXM

PRINT 14, DTPM, DHCMA, DHCMI, DOH, TMA XM

READ 3, SILIN,SILOUT,PHIIN,SMLIIN, TOTFI,CSII,TCPI, COPI

IF (PAIIN.NE.0.) PHI =PHIIN

IF (SMLIIN.NE.0.1 SMLI = SMLIIN

IF (TOPI.GT:0.) TCP $=$ TCPI

IF $(C D P I . G T .0,1) C D P=C D P I$

PRINT 6, SILIN, SILOUT,PHI, SMLI, TOTFI, CSII, TCP,CDP

IF(CSII.GT.0.) PRINT 13

PRINT 7

DO $2 K=1$. NREF

READ 3, TREFV (K), FLASHRV (K), TE PPRV (K), PHRV (K)

PRINT 8 , TREFV $(K), F L A S H R V(K), T E M P R V(K), P H R V(K)$

2 CONTINUE

TSTART = TREFV (1)

$K S T P=0$

IF (NCLC.LE.O) GO TO 3

PRINT 10

DO $4 . K=1$, NCLC

READ 3, CLR, CLM

$C L R=2 L R * 1 E-7$

$P M=P\left[4 / 3 . * D E N S M^{*} C L R * * 3\right.$

CLNV $(K)=C L M / P M$

CLMV $(K)=C L M$

CLRV $(K)=C L R$

PRINT $11, K, C L R, C L M, C L N V(K)$

4 CONTINUE

9 CONTINLE

CALL SETUP(IBORT)

IF(IBORT.GT.O) GU TO 5

CALL MASTER

5 CONTINUE

1. FORMAT (16I5)

3 FORMAT ( $8 F 10.6)$

6 FORMAT $(*$ SILIN,SILOUT $=*, 2 F 8,4,3 X, *$ PHI, SMLI $=*, F 8,4,1 P E 12,4,3 X$,

1 *TOTFI $=*, 1 P E 12.4 / 1^{*}$ CSII $=*$, OPF $8,4,3 X_{1}^{*}$ TCP $=*, 1 P E 12,4,3 X_{*}$

$2 *$ COP $=* . O P F B .4 / 1$

7 FORMAT $\left(6 x^{*}, *\right.$ TRE F*, 5X,*FLASH*,6X, *TEMP*, 8X,*PA*/)

6 FORMAT (1X,4F10.4)

10 F ORMAT (1HB, 3X,*K*,6X,*RADIUS*, 8X, *MASS*,6X,*NJMBER*/)

11 FORMAT $(1 X, I 5,1 P 3 E 12,4)$

12. FORMAT (1H:1,*NREF, NCLC, INX =*,I3,I 4,I3,2X, "IPR,IPR2 =*,2I3, 2X,

$1 * K D$, NDP $=*, 2 I 4,2 X, *$ I TPM, IMAX $=*, I 3, I 5,2 X, *$ IHCMA,IHCMI,IDH, IMAXM $2=*, 4[3 / 1$

13 FORMAT (* MONOSILICIC ACIO BUFFERING IS ASSUYEJ * 11

14 F ORMAT (1X,*DTPM $=*, 1 P E 11,3,3 X, *$ DHCMA, DHCMI =*, 1P2E $11,3,3 X$,

$1 * O D 1, T M A X M=*, 1 P 2 E 11,3 / 1$

END 
SLBRJUTINE MASTER

LOGICAL LTH, LTO,LNSC

COMMON /CALCSIL/ CSILV (3), CFIM(3,3),TCV $(3), C S F V(3)$

COMMON ICLVAR/ CL DV (1200),CLMV(300), CLNV $(300)$, CLRV $(600), N C L C, D C S I L$

COMMON /CVALS/ PI, PI4, DENSN,DENSM,UMASS, CKA, ALN 10 , A VN,S ILMW

COMMON /KINCON/ HCMA, HCMI, TCMA, EPSIL,EPR, KRG C, IMAX, RL IM, ICCL, RRMN

COMMON INUCL/ FNCAP,ZLD, CN, QCAP,QT, RNUC, ANNUC, TAUC, RANUC, QLP

COMMON /PRINOUT/ NDP, DTF,TPN,KPN, IPR,IPR2,TCP,CLP,KLP,CDP,KDP

COMMON /SETPAR/ DHCMA, CHCMI, DOH,TMAXM, OTPY

COMMON ISILKIN/RA TEK, RCF, R CEPF, FKINL, CSILS, SRA, SRR, CLAST,AKIN, DKIN

COMMON /SPECIN/ TSPEC, TEMP, TEMPL, FLASH,GLASH,FLC, FLCL

COMMON /START/ SILIN,SILOUT, ICX,INX, TSTART, TAJCS

COMMON /STEPS/ KSTP,THL, DELTC, DEL TH,LTH,LTD,LNSC

CSF $(O T)=\operatorname{CSFV}(1)+\operatorname{CSFV}(2)+D T+C S F V(3) * D T * D T$

PRINT 102

RNMX $=0$.

LNSC $=$.TRUE.

IF (IPR.GË.2) CALL OUTPUT

PRINT 1

DO 4 KST $=P=1$, I MAX

KSTP $=$ KSTEP

CALL DEPOSIT (ILOSS)

IF (ILOSS.LT.O) GO TO 5

IF (ILOSS.NE * O) : LNSC =. TRUE.

IF (ILOSS.GT.0) GO TO 39

IF(IVX.EQ.0) 60 TO 39

$T A U=1.08 E-6 / R D E P F *(Z L D+Q T * R N U C * R N U C) *(-0.25)$

$D H N=T A U / D O H$

21 CONTINUE

$T H N=T H L+D E L T H$

IF (DHN.GE.DELTH) GO TO 15

$T H N=T H L+0.5 *(D E L T H+U H N)$

IF(TIN.GT.TCV(1)) GO TO 39

DEL TH= DHN

$T A U C S=T A \cup C$

IF(IPR.GE.3) PRINT 19, DELTH,TAUCS, TCV (1), THN

IF (IPR.GE.3) PRINT 1

15 CONTINUE

IF $T+N \cdot G T \cdot T C V(1))$ GO.TO 39

$T H L=T H N$

IF(NCLC. $\equiv Q .300) \quad 60$ TO 38

TSPEC $=$ THL

CALL SPECIFY

LNSC = .TRUE.

DT $=T H L-T C V(1)$

$C S I L=C S F(D T)$

$K R G C=0$

CALL SILKIN(CSIL)

$A N=A N N U C+0.5 / Z L D$

$A R G=3 . /(P I 4 * 0 E N S N) * A N$

$C L R=E X P(A L O G(A R G) / 3$.

CF $=1 .-\triangle X P(-$ (THL-TSTART ITAUCS)

RANUCC $=$ RANUC+CF

$C L N=Z A N U C C * D E L T H$

IF (RANUCC.GT.RNMX) RNMX=RANUCC

IF(RANUCC.LT.RRMN*RNMX) GO TO 37

$N C L C=N C L C+1$

$\operatorname{CLRV}(N C L C)=C L R$

CLNVINCLC) $=C L N$

CLM $=P I 4 / 3$ * DENSM*CLR* *3

$C L M=2 L M+C L N$ 
CLMV $(N C L C)=C L M$

CSILV $(1)=C S I L V(1)-C L M$

IF(IPR.LT.5) GO TO 27

PRINT 2

PRINT 17,KSTEP, CLR, CLN,CLM, THL, NCLC

27 CONTINUE

CS1=CSILV (1)

CS2 $=$ CSILV $(2)$

CS3 $=$ SSILV $(3)$

$\operatorname{CSFV}(1)=\operatorname{CFIM}(1,1) * \operatorname{CS} 1+\operatorname{CFIM}(1,2) * \operatorname{CS} 2+\operatorname{CF} \operatorname{IM}(1,3) \cdot \operatorname{CS} 3$

$\operatorname{CSFV}(2)=\operatorname{CFIM}(2,1) * \operatorname{CS} 1+\operatorname{CFIM}(2,2) * 3 S 2+\operatorname{CFIM}(2,3) * \operatorname{CS} 3$

$\operatorname{CSF} V(3)=\operatorname{CFIM}(3,1) * \operatorname{CS} 1+\operatorname{CFIM}(3,2) * 3 \operatorname{S} 2+\operatorname{CF} \operatorname{IM}(3,3) * \operatorname{CS} 3$

GO TO 21

39 CONTINUE

IF(.NOT.LTH) GO TO 12

DELTC $=0.5 *$ DELTC

IF(IPR,GE,3) PRINT 13,DELTC,KSTEP

IF (IPR.GE.3) PRINT 1

$I C C L=K S T \equiv P$

GO TO 16

12 CONTINUE

IF (.NOT. (LTO.AND. 1.99*DELTC.LE.HCMA)) GO TO 15

IF (INX.GT.0.AND.DELTC.GT.0.5*DELTH) GO T0.15

DELTC $=2 *$ DELTC

IF(IDR.GE, 3$)$ PRINT 13, DELTC,KSTEP

IF(IPR.GE 3 ) PRINT 1

I CCL $=K S T E P$

16 CONTINUE

IF(CSILV(1).LT.SILOUT) GO TO 9

IF (KSTEP.GE.IMAX) GO TO 6

IF(TCV (1)+DELTC.GT.TCMA) GO TO 32

IF (ILOSS.GT.O) GO TO 43

GO TO 42

37 CONTINUE

IF (IVX.SQ.0) GO TO 42

PRINT 3

PRINT 41 .RANUCC,RRMN,RNMX

IF(IPR.GE.2) CALL OUTPUT

I $N X=3$

GO TO 28

38 CONTINUE

IF IIVX.EQ.0) $60: 1042$

PRINT 3

PRINI 40

IF (IPR.GE.2) CALL OUTPUT

$I N X=0$

GO TO 28

43 CONTINUE

IFIINX.EQ.0) 60 10 42

PRINT 3

PRINT 11

IF (IPR.GE.2) CALL OUTPUT

I $N X=0$

GO TJ 28

9 CONTINUE

PRINT 20

PRINT 10. SILOLT

IF(IPR.GE. 2$)$ CALL OUTPUT

$I C X=0$

GO TJ 28

32 CONTINUE

PRINT 25 
PRINT 33

IFIIPR.GE.2) CALL OUTPUT

$I C X=0$

GO TO 28

6 CONTINUE

PRINT 26

PRINT 35

IF(IPR.GE.2) CALL OUTPUT

I $C X=$ ]

GO TO 26

5 CONTINUE

PRINT 7

PRINI 14

I CX $=0$

IF(IPR.GE.2) CALL OUTPUT

28 CONTINUE

IF(I'R,LT,2) PRINT 36,KSTEP,TCV(1),CSILV(1)

IF IISX.GT.0) PRINT 1

42 CONTINUE

IF $(I 3 X, E Q \cdot 0)$ GC TO 8

IF (TOV (1).GT.TCP) I PR = IPR2

IF (IPR.LT.4) GO TO 4

IF (KSTEP.GE.KPN) CALL OUTPUT

IF (TCV (1).GE.TPN) CALL OUTPUT

4 CONTINUE

8 CONTINUE

1 FORMAT (* KSTEP*, $5 X, * T I M E *, 3 X, * C S I L *, 4 X, * S R R^{*},+X, * F L C *, 3 X, * T E M P *$,

$14 X,{ }^{*} P H^{*}, 3 X,{ }^{*} P H N^{*}, 4 X_{1}{ }^{*} F P H^{*}, 6 X, * R D E P^{*}, 3 X,{ }^{*} \mathrm{NUC}^{*} \mathrm{RATE}^{*}, 6 X_{,}^{*} \mathrm{CAREA}^{*}, 3 X$,

2 *NU2,FLLX*, 3X,*DEP.RATE*,6X,*ALPSIL*/1

2 FORMAT $26(*$ NUC * $1 /)$

3 F ORMAT $(/ / 161 *$ ENDNUC *1/)

7 FORMAT $(/ / 13(*$ ALL GONE *)/)

10 FORMAT (* SILICA. CONCENTRATION HAS OROPPED BELOH SILOUT =* .F8.4/1

11 FORMAT (* OENUCLEATION STARTED AND NUCLEATION ENDED*/)

13 FORMAT $1 * O N E W$ OELTC $=*$, 1PE 12.4,* AT KSTEP $=*$, IJ/I

14 FORMAT * ALL COLLOID CLASSES HAVE OISSOLVED AWAY. PROBLEM ENDED. $1 * / 1$

17 FORMATI* A NEW COLLOID CLASS HAS NUCLEATED AT KSTEP $=*$, I5/

1 * RAOILS, NUMBER,TOT.MASS =*, 1P 3E $12,3,5 X$, "TIME =*, 1 PE $12,3,3 \mathrm{X}$.

2 *NCLC $=*, I 5 / 1$

18 FORMAT $11 X, * C S I L, A N N U C, A N={ }^{*} F 8.4,2 F 8.1,3 X,{ }^{\circ}$ NUL,PF, S.S.RATE, CORR,RA 1TE $=*$. 1P3E13.4,3X,*CORR. FACT CR $=*, 0 P F 3.411$

19 FORMAT (*OHEW DELTH, TALCS $=4,1 P 2 \equiv 12.4,3 X, * A T$ TOV(1), THA $=*, 1 P 2 E 12.4$ 111

20 FORMAT $(/ / 161 *$ SILOUT * $/ 1)$

25 F ORMAT $(/ / 16(*$ T IHEX * $1 / 1)$

26 F CRMAT (/118(* CONEX * //)

33 F CRMAT ( * MAXIMUM TIME RËACHED*/)

35 FORMAT ( * MAXIMUM NUMBER OF CONDENSATION STEPS COMPLETED*

36 FORMAT (* KSTEP $=*$, I5, $3 \lambda^{*},{ }^{*}$ TCV $(1)=*, 1 P E 12,3,3 X_{0} *$ CSIL $=*, 0 P F B, 4 / 1$

40 FORMAT 1* MAXIMUM NUMBER OF COLLOIO CLASSES PEACHED. NUCLEATION END 1ED.*/1

41 F ORMAT (* PRECEEOI NG NUCLEAT IOA RATE $(*, 1$ PE $12,4, *)$ LESS THAN $(*$,

1 1PE $12.4, *)$ OF RECORDED MAXIMUM RATE $(*, 1 P E 12.4, *)$. NUCLEATION END 2E $0^{*} / 1$

102 FORMAT $(/)$

RETURN

END 


\section{SUBROUT INE SETUP(IBORT)}

LOGICAL LTH,LTO,LNSC

COMMON /CALCSIL/ CSILV(3), CFIM $(3,3), \operatorname{TCV}(3), \hat{S S F V(3)}$

COMMON /CLVAR/ CLDV (1200), CLMV 300$),$ CLNV $(300)$, CLRV $(600), N C L C, D C S I L$

COMMON /CVALS, PI, PI4, DENSN, DENSM, UMASS, CKB, ALN 1O, AVN, S ILMW

COMMON /GAMMA/ GAMC,GAMT,GAMZ,GAMMA,CAREA

COMMON /KINCON/ HCMA, HCMI, TCMA,EPSIL,EPR, KRGC, IMA X,RLIM,ICCL, RRMN

COMMON INUCL/ FNCAP,ZLD,CN,QCAP,QT,RNUC,ANNUC, TAUC, RANUC,OLP

COMMON /PRINOUT/ NDP,DTP,TPN,KPN, IPR, IPR2,TCP,CLP,KLP,CDP,KDP

COMMON /SETPAR/ OHCMA, OHCMI, DOH, TMAXM, OTPM

COMMON /SILKIN/RATEK, RCF, RCEPF, FK INL,CSILS,SRA, SRR, CLAST,AKIN,DKIN

COMMON /SPECIN/ TSPEC, TEMP, TEMPL, FLASH, GLASH,FLC, FLCL

COMMON /SPECPAR/ NREF, ICALL,I 1,I2,T1,T2

COMMON /SPECS/ TREFV $(50)$, FLASHRV $(50)$, TEMPRV $(50)$, PHRV (50)

COMMON /START/ SILIN, SILOLT, ICX, INX, TSTART,TAJCS

COMMON /STEPS/ KSTP,THL, DELTC, DELTH,LTH,LTO, LNSC

IF (NCLC.LE. O.AND.INX,EQ.0) GO TO 9

LNSC $=. T R U E$.

$I B O R T=0$

$I C A L L=0$

$I C C L=0$

$K P N=0$

$C L P=1000$.

$K L P=-100$

$K R G C=0$

TSPEC $=$ TSTART

$P R E R=3 . /(4$. *PI*DE NSN)

1 CONTINUE

$T A U D=1 E 80$

$T A U N=1 E 80$

CALL SPECIFY

CSIL $=$ SILIN*FLC

CALL SILKIN(CSIL)

$T E S T=Z L D * Q T+R N U C *$ FNUC

IF(TEST.GT.0.) GO TO 2 .

IF (INX.EQ.0.AND.SRR.GT.1.) GO TO 2

5 CONTINUE

IF (NREF.LE. 1) GO TO 7

TSTART $=T 2$

TSPEC $=$ TSTART

IF (TSPEC, GE, TREFV (NREF)) GO TO 7

GO TJ 1

2 CONTINUE

IF (INX.NE.0) TAUN $=1.08 E-6 / R O E P F * T E S T *(-0.25)$

IF(NCLC.LE.O) GO TO 11

CAREA $=0$.

DO $12 K=1, N C L C$

$C R=C L R \vee(K)$

IF (CR.GT.RNUC) CAREA =CAREA+CR *CR*CLNU (K)

12 CONTINUE

CAREA = CAREA *PI4*FLC

IF (CAREA.GT.0.) TAUD = (CSIL-CSILS)/ (CAREA*RDEPF)

11 CONTINUE

TAU $=$ TAUN

IF (TAUD.LT.TAUN) TAU = TALD

IF (TALF,GT.9.9E79) GO TO 5

OELTH $=T A U / D O H$

IF(NREF.LE.1) GO TO 4 
IF(DELTH.GT.(T2-T 1)) GOTO 5

4 CONTINUE

IHL $=A L O G(T A U) / A L O G(2.1+0.5$

RTAU $=2 * *$ IHL

HCMA = RTAU/DHCMA

HCMI = RT AL / DHCMI

DTP=OTPM* IAU

TCMA $=$ TAU*TMAXM

IF (NREF •GT •1॰AND.TCMA •GT.TREFV (NREF) ) TCMA=TREFV (NREF)

$K N U C=A N N U C+0.5$

$A N=A N N L C+0.5 / 2 L D$

RLIM $=E \times P(A L O G(P R E R * A N) / 3$. $)$

TPN $=$ TSTART

$T H L=$ TSTART

PRINT 14, KNUC, ANNLC,AN, ZLD, RL IM

NCLC $=$ NCLC +1

$C L N V(N C L C)=1$.

$C L R V(N C L C)=R L I M$

CSILV $(1)=C S I L$

TALCS $=$ TALC

PRINT 15, TAUN, TAUD, TAL,RTAL

PRINT 6,HCMA,HCMI, OLLTH,TCMA, OTP, NDP

PREC $=P I 4 / 30^{*}$ CENSM

CAREA $=0$.

DO $3 K=1$, NCLC

$C R=C L R V(K)$

CLNV $(K)=C L N V(K) * F L C$

$I F(K . E Q . N C L C) \quad C L N V(K)=1$.

$C L M V(K)=P R E C * C R * 3 * C L N V(K)$

$C A R E A=C A R E A+P I 4 * C R * C K * C L N V(K)$

IF $(K \cdot L T \cdot N C L C)$ SILIN=SILIN+CLMV(K)/FLC

3 CONTINUE

$F L C L=F L C$

$\operatorname{CFIM}(1,1)=1$

$\operatorname{CFIM}(1,2)=0$.

$\operatorname{CFIM}(1,3)=0$.

DELTC $=H C M I$

$\operatorname{TCV}(1)=T S T A R T$

TCV $(2)=T 3 T A R T-D E L T C$

IF I INX.EQ.0) RETURN

$T K B=C K B^{*}(T E M P+273 \cdot 15)$

$D E R=1 . /\left(O E N S N^{*} T K B * A L O G(S R R)\right)$

$D E R=+\infty * P I /(3 \bullet * T K E) * D E R * D E R / A L N 10$

DERI $=1 . / 0 E R$

GAMCUB $=$ GAMMA**3

PRINT 13, OER, DERI, GAMMA, GAMCUB

RETURN

7 CONTINUE

IBORT $=1$

PRINT 8

RETURN

9 CONTINUE

$I B O R T=1$

PRINT 10

RETURN

6 FORMAT (* HCMA,HCMI =*, 1P2E12, 4,3X,* CELTH, TCMA =*, 1P 2E $12,4,3 X$,

1 *DTP, NDP $=*, 1 P E 12,4, I 5 / 1$

6 FORMATI* INITIATION CONDITICNS NE VER REACHED. PROBLEM EEING ABORT IED.*/1

10 FORMATI* NO CULLOID ADDEO AND NUCLEATION NOT SPECIFIEO. PROBLEM B IING ABORTED.*/1

13 FORMAT $1 * 0$ LOG10 TAL/D GAMMA $3=*, 1 P E 13,5,3 X, * R E G I P R O C A L=*$. 
1 1PE13.5,3X,*GAMMA $=*$, OPF 7.2,3X,*GAMMA CUEED $=*, 1$ PE $13.5 / 1$

14 FORMAT $(1 x / *$ KNUC $=*, I 5,3 X, * A N A U C, A N=*, 2 F 8,1,3 X, * Z L D, R L I M=*$,

$11 P 2 E 13 \cdot 4 / 1$

15 FORMAT (* TAUN,TAUD $=*, 1 P 2 E 13,4,3 X, *$ TAU,RTAJ $=*, 1 P 2 E 13,4 / 1$

END

SLEROUTINE DEPOSIT (ILOSS):

LOGICAL LTH,LTO,LNSC

COMMON /CALCSIL/ CSILV $(3), \operatorname{CFIM(3,3),TCV(3),GSFV(3)~}$

COMMON /CLVAR/ CLOV $(1200), C L M V(300), C L N V(300), C L 2 V(600), N C L C, D C S I L$

COMMON /CVALS/ PI,PI4, OENSN, DENSM, UMASS, CKS, ALN $10, A$ VH, S ILMH

COMMON /GAMMA/ GAMC,GAMT, GAMZ,GAYMA, CAREA

COMMON /KINCON/ HCMA,HCMI, TCMA,EPSIL,EPR,KRJC, IMAX, RLIM, ICCL, RRMN

COMMON /NUCL/ FNCAP,ZLD,CN,QCAP,QT,RNUC, ANNUC, TAUC, RANUC, QLP

COMMON /PH/ PH,PHL, PHN,PHNL,AH,FPH, FINT, OQS, DSAMQ,FF

COMMON /ORINOUT/ NDP, OTP,TPN,KPN, IPR, IPRZ, TCP, CLP, KLP,C GP, KDP

COMMON /RKCS/ RK21,KK22,KK31,RK32,RK41,RK42

COMMON /SALT/ PHI, SML, SMLI, GNA, ANA, AKSIL, ALPSIL, SCR,GSIL,GH

COMMJN /SILKIH/RATEK,KCF,RCEPF,FK INL, CSILS,SRA,SRR, CLAST,AKIN, OKIN

COHMON /SPECIN/ TSPEC, TEMP, TE MPL, FLASH,GLASH,FLC, FLCL

COMMON /START/ SILIN,SILOLT,ICX,INX,TSTART,TAJCS

COMMON /STEPS/ KSTP,THL,OELTC, OELTH,LTH,LTO,LNSC

DIMENSION OCSV (4)

NCND $=K S T P$

CSIL $=$ CSILV(1)

CSILN $=$ CSIL

TSPEO $=T C V(1)$

DO $7 \mathrm{~K}=1,4$

$K R G C=K$

IF (KRGC. $=Q_{0} 1.0 R_{0} K R G C_{0}=Q_{0.3)}$ GO TO 38

TSPEC $=T S P E C+0.5 * D E L I C$

CALL SPECIFY

38 CONTINUE.

DCSIL $=C S I L N * G L A S H * D E L T C$

DCS $=$ JCSIL

CALL SILKIN(CSILN)

$\operatorname{DCSV}(K)=J C S I L$

IF (KRGC.EQ.1)

$I F(K R G C, E Q .2) \quad C S I L N=C S I L+K K 22 * D C S V(1)+R K 22 * J C S I L$

IF (KRGC.EQ.3) CSILIV=CSIL+RK $31 * O C S V(2)+R K 32 * D C S I L$

7 CONTINUE

$\operatorname{CSILN}=D \operatorname{CSV}(1)+R K 41+D \operatorname{CSV}(2)+R K 42 * D \operatorname{CSV}(3)+D \operatorname{CSV}(+)$

CSILN $=$ CSIL+CSILN/6.

$\operatorname{TCV}(3)=\operatorname{TCV}(2)$

$\operatorname{TCV}(2)=\operatorname{TCV}(1)$

$T C V(1)=T S P E C$

IF (NCND.GT.1) GO TO 11

$\operatorname{CSILV}(1)=\operatorname{CSIL}$

DELSIL $=$ CSILN-CSIL

$\operatorname{CSILV}(2)=$ CSIL-DELSIL

CSILV $(3)=$ CSILV $(2)-O L L S I L$

11 CONTINUE

CSILV $(3)=\operatorname{CSILV}(2)$

$\operatorname{CSILV}(2)=\operatorname{CSILV}(1)$

$C S I L=C S I L N$ 
CSILP $=$ CSILV (2)

TEST $=(C S I L-C S I L P) / C S I L P$

TESTH $=A B S(T E S T H)$

CSILV $(1)=C S I L$

LTH=TESTH.GT.EPSIL

LTO=?.*TESTH०LT.EPSIL

PREC $=P I 4 / 3 . *$ DENSM

SUMCA $=0$.

STEST $=0$.

ILOSS $=0$

$001+K=1, N C L C$

$C R=C L R V(K)$

IF (CR.EQ.0.) GO TO 14

$C L M=P R E C * C R * * 3 * C L A V(K)$

CLMV $(K)=C L M$

IF $(C R . G T .0 .2 * R L I M)$ GO TO 43

$C S I L=C S I L+C L M$

CSILV $(1)=C S I L$

$I L O S S=I L O S S+1$

IF(IPR.LT.5) 60 TO 3

PRINT 1

PRINT $44, K, C R, C L N V(K), C L M$

PRINT 36,KSTP,TCV (1), CSIL

PRINT 1

3 . CONTINUE.

$C R=0$.

CLNV $(K)=0$.

CLRV $(K)=0$.

CLMV $(K)=0$.

GO TO 14

43 CONTINUE

$K K=K+300$

$C R P=2 L R V(K K)$

TEST $=(C R-C R P) / C R P$

TESTH $=A E S(T E S T H)$

SUMCA $=$ SUMCW + CLM

$S T E S T=S T-S T+C L M * T E S T H$

14 CONTINUE

IF(S.JHCW.GT.0.) GO TO .2

ILOSS $=-1$

RETURN

2 CONTINUE

TESTA $=S T E S T / S U M C W$

LTHELTH.OR,TESTH,GT .EPR

$L T D=-T D \bullet A N D \cdot 2 * * T E S T H \cdot L T \cdot E P R$

IF (IPR.LT.1) GO TO 5

IF (CSILV(1), GT. (CLP-COP) A ND.NCND \&LT. (KLP+KDP) 50 TO 5

$A R G=-(T C V(1)-T S T A R T) / T A L C S$

RANUCC $=$ RA NUC* $(1 .-E X P(A R G))$

$F M N=P I 4 * D E N S M / 3 *$ RNUC* *3*RANUCC

$F M C=-(\operatorname{COSV}(4)-D C S) / D E L T C$

PRINT \& NCND, TCV(1),CSILV (1), SRR, FLC, TEMP, PH, PHN, FPH, ROEPF

1 , RAVUCC,CAREA, FMN, FMC,ALPS IL

RANUS =0.

CLP=CSILV(1)

KLP $=$ NCND

5 CONTINUE

IF (INX.EQ.0) RETURN

IF ( $(N C N D-I C C L), G E .3) . G O T O 17$

$T 2=\operatorname{TCV}(2)-T S P E C$

T3=TCV $(3)-T S P E C$

$D=T 2 * T 3 *(T 3-T 2)$ 
$\operatorname{CFIM}(2,1)=(T 2+T 2-T 3 * T 3) / D$

$\operatorname{CFIM}(2,2)=T 3 * T 3 / 0$

$\operatorname{CFIM}(2,3)=-T 2 * T 2 / 0$

$\operatorname{CFIM}(3,1)=(T 3-T 2) / 0$

$\operatorname{CFIM(3,2i=-T3/0}$

$\operatorname{CFIM}(3,3)=T 2 / 0$

17 CONTINLE

CSI $=$ SSILV(1)

$C S 2=2 S I L V(2)$

CS3 $=0 S I L V(3)$

$\operatorname{CSFV}(1)=C F \operatorname{IM}(1,1) * C S 1+\operatorname{CFIM}(1,2) * 3 S 2+\operatorname{CF} \operatorname{IM}(1,3) * 253$

$\operatorname{CSF} V(2)=\operatorname{CFIM}(2,1) * C 31+\operatorname{CFIM}(2,2) * \operatorname{CS} 2+\operatorname{CFIM}(2,3) * 0 S 3$

$\operatorname{CSF} V(3)=\operatorname{CFIM}(3,1) * \operatorname{CS} 1+\operatorname{CFIM}(3,2) * \operatorname{CS} 2+\operatorname{CF} \operatorname{IM}(3,3) * \operatorname{CS} 3$

IFIIPR.LT.6) RETURN

PRINT 1JU, NCNA,TCV,CSILV,CSFV

1 FCKMAT $191 *$ DENUC *)/1

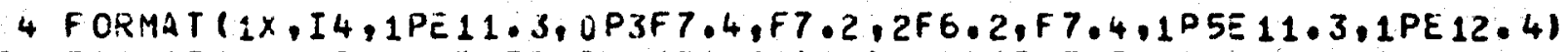

36 FORMAT (* KSTEP $=*$, IE, $3 X_{*} *$ TCV $\left.(1)=*, 1 P E 12,3,3 X, * C S I L=*, 0 P F 9,4 / 1\right)$

44 FOKMAT I* A COLLOID CLASS HAS DENJCLËATE. K,RADIUS, NUMBER, TOTAL $\mathrm{F}$ $1 A 3 S^{*}=$,IS, 1P3E12.4/1

100 FURMAT $(1 \times, 15,1$ PQE 13,4$)$

RLTLZN

ENO

SUERJUTINE SILKIN(CSIL)

LOGICAL LSKIP

LOGICAL LTH,LTO,LNSL

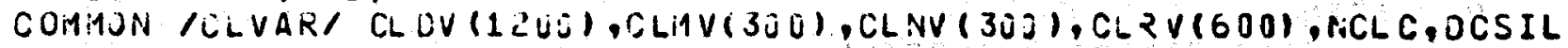

COMIUUN /CVALS/ PI,PI4, DENSN, OENSM, UMASS, CK3, GLN10, A VN, S ILMW

COMMON /.SAMMA/ GAMC, GAMT, GAMZ,GAMMA, CAREA

COMMJN /KINCCN/ HCMA, HCMI,TCMA,EPSIL,EPR,K ₹ C I, IMAX, RLIM, ICCL, RRMN

COMAON /NUCL/ FNCAP,ZLO,CN,QCLD, QT, KIUC, ANVUC, TAUC, RAAUC,QLP

COMIJN TPHI PH,PRL, PHN,PHNL,AH,FPH, FINT, OQS,DSAM2, FF

COMMUN /KKCS/ RK21,KK22,RK31,RK32, RK41,RK42

COM1ON /JALT/ PHI,SML, SMLI,GNA,ANA,AKSIL,ALPSIL,SCR,GSIL,GH

COMMON /SILEUF/ CSI,CSII

COMMON /SILKC/ ALPHAK,EK,AK,AKIND,AKINT,OKINC,OKINT

CGM:1ON ISILKF/ TOTFI, TUTF,PKF,RKF I,KKF2,ALDF, RATEKF,RATEKT,GF

COMMON /SILKFC/ CKF 1,TKF1, CKFZ,TKFZ

COMMUN /SILKIN/RATEK,F.CF, RDEPF, FK INL,CSILS,SRA,SRR, CLAST,AKIN, OKIN

COMMOIN /SPECIN/ TSPLC, IEMP,TEMPL, FLASH,GLASH,FLC, FLCL

COMMON /STEPS/ KSTP,THL,OELTC, JEL TH, LTH,LTO,LNSC

COMMUI /NATER/ DENS,EPSD,AOH, DDH.PKN,TK.ALNT,T<O

$A L G 1 O(X)=X P\left(A L N 10^{*} \lambda\right)$

$T C=T E Y P$

$T K=T 2+273 \cdot 15$

$T K E=E K A^{*} T K$

LSKIP $=\angle R G C \cdot E Q \cdot 1.0 R . K F G C . E Q .3$

IF (LNSC) LSKIP= OFALSE .

IF $(K<G C .=Q .1) \quad \angle N S C=$.FALSE.

IF(LSKIP) GO TO

IF(TEMP.LQ.TEIAPL) GL TO 13

ALAT $=A L O S$ (TK)

CALL WAT:R 


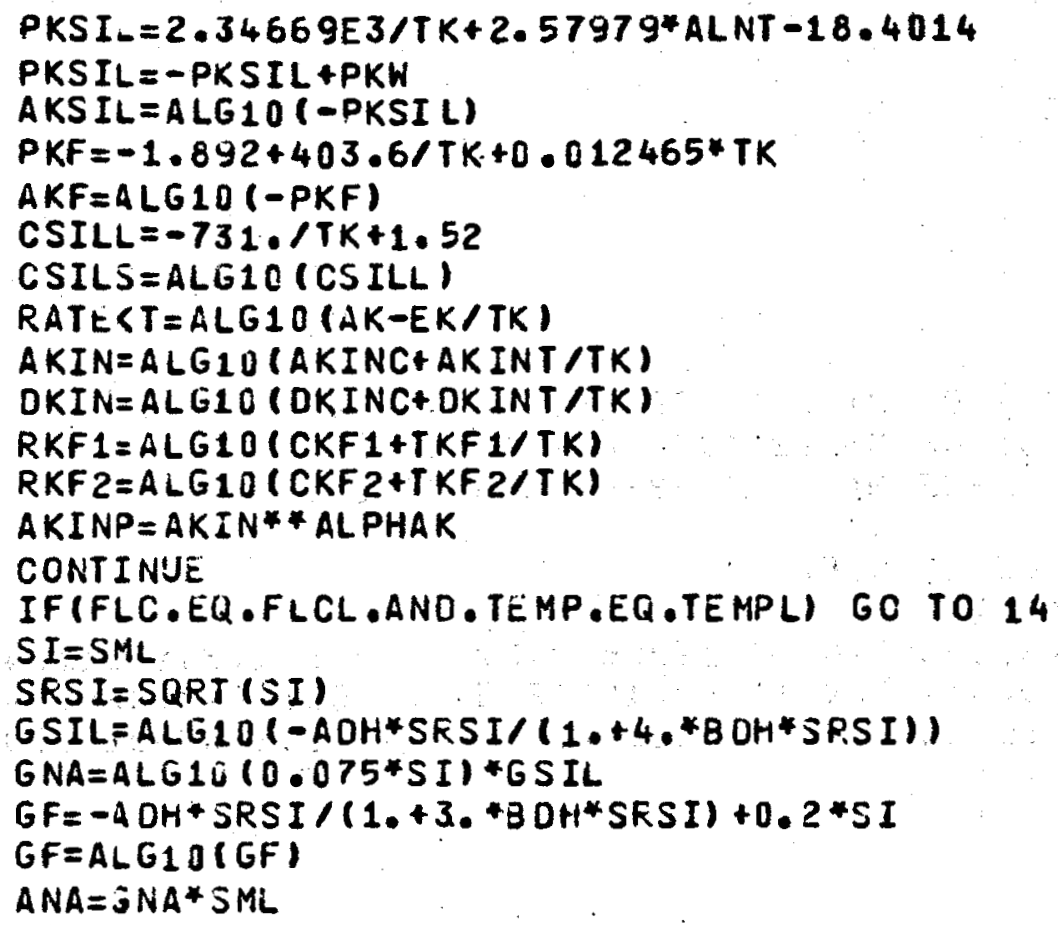

1 CONTINUE

$A L P F=1 \bullet /(1 \bullet+A H * G F / A K F)$

$P H N=P H+A L O G 10(\cdot A N A / 0.069)$

CALL PHF

RATEKF $=$ TOTF* $(R K F 1 *(1 .-A L P F)+R K F 2 * A L P F)$

RATEK =RATEKT* (FPH +RATEKF)

9 CONTINUE

$S R A=C S I L / C S I L S *(1,-A L P S I L)$

$G A M Z=G A M C+G A M T * T C$

DGAMQ $=-A L N 10 * D Q S^{*} T K E^{*} F$ INT

GAMMA $=$ GAMZ+DGAMQ

$S C R=E X P(0.036032 * P H I+S M L)$

$S R R=S R A * 3 C R$

$S R R L=A L O G(S R R)$

SRAL $=A L C B(S R A)$

FKINL $=2$ * GAMMA/(OENSN*TKB)

IF (SRA •LT •AKIN) RCF=RATEK*EXP (ALPHAK*SRAL) IF (SRA.LT.AKIN) GO TO 7

RCFA = RATCK*AKINP

ORCFA $=R C F A * A L P H A K / A K I N$

$D I F=S R A-A K I N$

$R C F=D R C F A * D I F+R C F A$

7 CONTINUE

$R D E P F=R C F *(1,-1, / S R R)$

IF (SRR.LT.1.) RCF $=0$.

IF (SRR . LT . 1.) RDE FF =RATEK*DKIN* $(S R R-1$.)

RCFN=RCF /UMASS

IF(KRGC.GT.O) GO TO 15

IF(SRR.GT.1.) GO TO 2

RNUC $=0$.

$\triangle N \cup C=0$.

$A N N U C=0$.

$Z L D=3$.

$Q T=0$. 
RANUS $=0$.

TAUC $=0$.

RETURN

2 CONTINUE

RNUC $=$ FK INL/SRRL

ANUC $=4 . * P I *$ RNUC*RNUC

FNCAP $=A N L C * G A M M A / 3$.

QCAP $=$ EXP(-FNCAP/TKB)

ANNUC $=P I 4 / 3 . *$ DE NSN* RNUC**3

$Z L D=3 . /\left(P I 4 * D E N S N^{*} A N N U C * A N N U C\right)$

$Z L D=2.13 . * E X P(A L O G(Z L O) / 3$.

$Z L O=Z L D * S Q R T$ (GAMMA/TKB)

$C N=R C F N * A N U C$

$Q T=Q L P * Q C A P$

RANUS $=Z L D^{*} Q T * C N$

$T A U C=1 . /(4, * C N * Z L D * 2 L O)$

RETURN

15 CONTINUE

$K E A S E=300 *(K R G C-1)$

$F C R=F L C / F L C L$

DELDENS= DELTC/OENSM

CAREA $=0$.

DO $4 K=1$, NCLC

$C R=C L R V(K)$

IF (CR.EQ.0.) GO TO 4

$K 1=K+300$

$K 2=K+600$

$F O R L=F K I N L / C R$

$C L D=R D E P F$

IF (FJRL.GT.SRRL) CLDERATEK*OKIN*(SRR-EXP (FORL))

CLO $=$ CLD*DELDENS

CLOV $(K+K B A S E)=C L O$.

$C L N=C L N V(K) * F C R$

$D C S I L=D C S I L-P I 4 * C L N * C L D * D E N S M * C R * C R$

CAREA $=C A R E A+P I 4 * C L N * C R * C R$

IF (KRGC.NE.1) GO TO 10

$C L R V(K 1)=C R$

$C L R V(K)=C R+0.5^{*} \mathrm{CL} \mathrm{DV}(K)$

10 CONTINUE

IF (KRGC.EQ.2) CLRV(K) =CLRV(K1)+RK 21*CLDV $(K)+R K 22 * C L O V(K 1)$

IF $(K R G C \cdot E Q \cdot 3)$ CLRV $(K)=C L R V(K 1)+R K 31 * C L D V(K 1)+2 K 32 * C L O V(K 2)$.

IF $(K R G C . N E .4)$ GO TO 4

SUM $=C L D V(K)+R K 41 * C L O V(K 1)+R K 42 * C L O V(K 2)+C L D V(<+900)$

CLRV $(K)=C L R V(K 1)+S U M / 6$.

CLNV $(K)=C L N$

4 CONTINUE

IF $(K R G C \cdot E G .4) \quad F L C L=F L C$

RETURN

END 
$P H L=P H$

PHNL $=P H N$

$Y=P H$

$003 K=1,2$

$x=\gamma-7,6$

IF $(X \cdot G T \cdot 1.12915) \quad x=2.2563-X$

IF $(X, G T \cdot-1.63)$ GO TO 1

$F P H=A L G 1 \cup(X)$

FINT $=A L O F 10(1 .+6.2 * F P H) / 6.2$

$F P H=F P H /\left(1,+6.2^{*} F P H\right)$

GO TO 4

1 CONTINUE

$A R G=1 .+A L G 10(x / 2.113)$

$F P H=-X /(9.6538+X *(1.7901+4.1811 * X))$

$F P H=X-2.113 * A L O G 10(A R G)+F P H$

$F P H=A L G 10$ (FPH)

FINT $=-0.75924+0.58993 * x-0.11292 * x * x$

FINT $=A L G 1 C$ (FINT)

4 CONTINUE

IF(Y.LT.0.72915) GO TO 2

$F P H=1,-F P H$

$F I N T=F I N T+Y-8.72915$

2 CONTINUE

$F P H=F P H / L .11891321$

IF(K.GT.1) GO TO 3

FINTF $=$ F INT

$F P H F=F P H$

$Y=P H N$

3 CONTINUE

$F P H=F F+F P H F+(1-F F) * F P H$

$F I N T=F F * F I N T F+(1,-F F) * F I N T$

RETURN

END

SUBRJUTINE WATER

COMMON /WATER/ DENS, EPSO,ADH,BDH, FKH,TK, ALNT,TKB

DIMENSION AOV $(6)$

PKW $=34.9734 * A L N T-0.097611 * T K-606.522$

$P K W=P K W+(3.1286 E 4-2.17087 E 6 / T K) / T K$

$P K W=-P K W$

$T C=T K-273.15$

$T C A=0.01 * T C$

IF(TO.GT.150) GO TO 11

A DV $(1)=0.9998396$

$A D V(2)=13.224944 \equiv-1$

$A D V(3)=-7.922210 E-2$

$A D V(+)=-55.44846 E-3$

$A C V(5)=149.7562 E-4$

$A D V(6)=-393.2952 E-5$

$B D=13.159725 E-1$

GO TO 13

11 CONTINUE

$A O V(1)=1.40776 B$

$A D V(2)=-1.52729 E-2$

$A D V(3)=-4 \cdot 33281 E-2$ 
$A O V(+)=1.22917 E-2$

$A O V(5)=-2.34375 E-3$

$A D V(5)=2 \cdot 6 E-11$

$B D=0$.

13 CONTINUE

SUM $=4$ DV $(6)$

$0014 \quad K=1.5$

$K K=6-K$

SUM $=A D V(K K)+S U M^{*} T C A$

14 CONTINUE

DENS $=S U M /\left(1,+B 0^{*} T C A\right)$

IF(TS.GT.10C) GO TO 15

SUM $=9.398 E-4-1.410 E-6 * T C$

SUM $=-0.40008+S U M^{*} T C$

$E P S D=87.740+S U M * T C$

GO TO 16

15 CONTINUE

SUM $=0.1417 E-2-0.8292 E-6 * T K$

SUM $=-0.9297+$ SUM*TK

EPSO $=5321 . / T K+233.76+$ SUM*TK

16 CONTINUE

TEPSD $=$ TK*EPSD

SQRTE $=S Q R T$ (TEPSD)

SQROEN=SQRT (DENS)

$A O H=2.82483 E 6 *$ SQR OEN/ (TEPSD*SQRTE)

$B O H=50 \cdot 2916 *$ SQRDEN/SQRTE

RETURN

END

SUERJUTINE SPECIFY

COMMON /CLVAR/ CL DV(1200),CLMV(300),CLNV(300),CLRV(600),NCLC,DCSIL

COMMON /CVALS/ PI,PI4,DENSN,DENSM,UMASS, CK3, ALN $10, A V N, S$ ILMW

COMMON /PH/ PH, PHL,PHN,PHNL, AH, FPH, FINT, DQS, DJAMQ, FF

COMMON /SALT/ PHI, SML, SMLI,GNA, ANA,AKSIL,ALPSIL,SCR,GSIL,GH

COMMON /SILBUF/ CSI,CSII

COMMON /SILKF/ TOTFI,TOTF,PKF,RKF 1,RKF 2,ALPF,RATEKF,RATEKT, GF

COMMON ISPECIN/ TSPEC, TEMP, TEMPL, FLASH,GLASH,FLC, FLCL

COMMON ISPECPAR/ NREF, ICALL,I1,I2,T1,T2

COMMON /SPECS/ TREFV(50),FLASHRV (50),TEMPRV (50),PHRV (50)

COMMON /START/ SILIN, SILOUT, ICX, INX, TSTART,TAJCS

ALG $10(X)=E X P(A L N 10 * X)$

$I C A L L=I C A L L+1$

IF(SMLI.GT.0.) GO TO 7

SMLI $=-S M L I$

SMLR $=S M L I$

DO \& $K=1,3$

PHIVL $=(16.40+2.153 *$ SQRT (SMLI) $) * 0.001$

$C R F=1.003 /(1 .-P H I V L * S M L R)$

$S M L I=S M L R * C R F$.

6 CONTINUE

$S I L I N=S I L I N^{*} C R F$

SILOST = SILOUT*CRF

CSII $=$ CSI $i *$ CRF

TOTFI $=$ TOT FI*CRF

IF (NSLC.EQ.O) GO TO 7 
$002 K=1, N C L C$

CLNV $(K)=\operatorname{CLNV}(K) * C R F$

2 CONTINUE

7 CONTINUE

IF(NREF.GT.1) GO TO 6

IF (ICALL.GT.2) RE TUFN

IF(IOALL.EQ.1) GO TO 4

TEMPL $=$ TEMP

RETURN

4 CONTINUE

TEMP = TEMPRV (1)

TEMPL $=-1000$

$P H=P$ HRV (1)

FLAS $1=F L A S H R V(1)$

GLAS $1=0$.

$F L C=1 . /(1,-F(A S H)$

$F L C L=F L C$

$S M L=S M L I * F L C$

TOTF $=$ TOTFI*FLC

CSI $=$ :SII*FLC

$A H=A . G 10(-P H)$

RETURN

6 CONTINUE

IF(IZALL.GT.1) GO TO 9

TEMP $=-1000$.

$I 1=0$

$I 2=1$

$T \dot{2}=-1=10$

$F L C L=1$.

9 CONTINLE

1 CONTINUE

IF(TSPEC.LT.TZ) GO TO 3

$I 1=I 1+1$

$I 2=I 2+1$

T $1=T R E F V\left(I_{1}\right)$

T $2=T R E F V(I 2)$

GO TO 1

3. CONTINUE

TEMPL $=$ TEMP

TFAC $=(T S P E C-T 1) /(T 2-T 1)$

TEMP $=$ TEMPRV $(I 1)+(T E M P R V(I 2)-T E M P R V(I 1)) * T F A C$

PH=P $\{R V(I 1)+(P H R V(I 2)-P H R V(I 1)) * T F A C$

FLÀSH=FLASHRV $(I 1)+($ FLA SHRV $(I 2)-F L A S H R V(I 1) *$ TFAC

GLASH $=($ FLASHRV (I2) -FLASHRV $(I 1)) /\left(T_{2}-T_{1}\right)$

$F L C=1 \bullet /(1,-F L A S H)$

GLAST=GLASH*FLC

$S M L=S M L I * F L C$

TOTF $=$ TOTFI*FLC

CSI $=$ SSII*FLC

$A H=A, G 10(-P H)$

RETURN

END 
COMMJN /CALCSIL/ CSILV $(3), C F I M(3,3), T C V(3), C S F V(3)$

COMMON /CLVAR/ CLOV (1200), CLMVP 3001, CLNV $(300)$, CLR V $(600)$, NCLC,DCSIL

COMMON /CVALS/ PI, P I 4, OENSN, DENSM, UMASS, CKB, ALN $1 O$, A VN, S ILMH

CCMMJN IGAMMA/ GAMC.GAMT, GAMZ,GAMMA,CAREA

COMMON /KINCON/ HCMA,HCMI, TCMA,EP $I L, E P R, K R G C$, IMA X,RLIM,ICCL, RRMN

COMMON INUCL/ FNCAP,ZLO,CN,QCAP,2T,RNUC, ANNUC,TAUC, RANIC,QLP

COMMON IPH/ PH,PHL, PHN,PHNL,AH,FPH, FINT, DQS, DJAMR,FF

COMMON /PRINOUT/ NOP,DTP,TPN,KPN, IPR, IPR2,TCP, CLP,KLP,CCP,KDP

COMMON /SALT/ PHI, SML, SMLI, GNA, ANA, AKSIL, ALPSIL,SCR,GSIL,GH

COMMON /SILBUF/ CSI,CSII

COMMON /SILKF/ TOTFI, TOTF,PKF,RKF 1,RKF2, ALPF, RATEKF, RATEK T, GF

COMMON $1 S$ ILKIN/RA TEK, RCF, RDEP F, FK INL, CSILS,SRA, SRR, CLAST, AKIN, OKIN

COMMON /SPECIN/ TSPEC, TEHP, TE MPL, FLASH,GLASH,FLC, FLCL

COMMJN /START/ SILIN, SILOUT,ICX, INX,TSTART,TAJCS

COMMON /STEPS/ KSTP,THL, DELTC,DEL TH,LTH,LTO,LNSC

CGMMON /WATER/ DENS, EPSD, ADH,BDH, PKH,TK, ALNT, T $\angle B$

TSPE C $=$ TCV $(1)$

$K P N=K S T P+N D P$

TPN=T SPEC+DTP

CALL SPECIFY

CSIL $=$ CSILV $(1)$

KRGC $=0$

CALL SILKIN (CSIL)

PRINT 100

PKSI $=-A L U G 10$ (AKS IL)

PRINT 2,KSTP,TSPEC,TEMP,PI, FLASH, GLASH,CSIL, FLC

PRINT 22,SML, SCR, ANA, PHN,FPH, FINT

PRINT $3, G A M Z, G A M M A, D Q S, A K I N, O K I N$

PRINT 23,TOTF,ALPF, KKF 1,RKF2, R.ATEKF, RATEKT

PRINT 24, PKW, PKSIL, PKF, GSIL, GF, GNA, EPSO, OENS, ADH, BDH

PRIIVT 4.ESILS,ALPSIL, CSI, SRA, SRR, RATEK, RDEPF, FKINL

PRINT 5,RNUC,ANNUC, ZLD,CN,RLP,QT, RANUC,TAUC

IF $(I: X . E Q .0)$ GO. TO 1

SUMN $=0$.

SUMR $=0$.

CAREA $=j$.

$A M C=3$.

DO $13 \mathrm{~K}=1$, NCLC

$C R=C L R \vee(<)$

$C L N=Z L N V(K)$

SUMN $=$ SUMN+CLN

SUMR = SUMR +CLN*CR

$C A R E A=C A R E A+P I 4 * C R * C R * C L N$

$A M C=A M C+C L M V(K)$

13 CONTINUE

RCAV = SUMR/SUMN

ACAV = CAREA/SUMN

CMAV = AMC/ SUMN

$A M T=S I L I N * F L C$

AMT $C=C S I L+A M C$

DELM=AMTC-AMT

PRINT 14, NCLC, SUMN, CAKEA, RCAV, ACAY, CMAV

DELM $=D E L M / A M T * 100$.

PRINT 21, AMC, CSIL, AMT C,AMT, OELM

PRINT $16,(C L N V(K), K=1, N C L C)$

PRINT 12

PRINT $17,(C L R Y(K), K=1, N C L C)$

PRINT 12

PRINT 18, (CLMV $(K), K=1, N C L C)$

PRINT 12

1 CONTINLE

PRINT 100 
2 F ORMAT (* KSTEP =*, I5, 3X, *TSPEC, TE MP, PH =*, 1PE12,4, OPF 8, 2,F 7, 2, 2X,

1 *FLASH,GLASH $=*, F B .4,1 P E 12.4,3 X, * C S I L=*, 0 P F 8,4,3 X, * F L C=*, F 8.4 / 1$

3 FORMAT $1 *$ GAMZ, GAMMA $=*, 2 F 6,2,2 X, * D Q S=*, 1 P E 10,3,2 X, *$ AKIN,OKIN $=*$.

1 OP2F7.3/1

4 FORMAT $*$, CSILS,ALPSIL, CSI $=*, 3 F 7,4,2 X, * S R A, S R R=*, 2 F 8,4,2 X$,

1 *RATEK,RDEPF =*, IPZE $12.4,3 X, * F K I N L=*, 1 P E 12.4 / 1$

5 FORMATI* RNUC, ANNUC $=*, 1 P 2 E 10,3,2 X, * Z L 0, C N=*, 1 P 2 \equiv 10,3,2 X$,

$1 * Q L P, Q T=*, 1 P 2 E 10,3,2 X, * R A N C=*, 1 P E 10,3,2 X, *$ TAUC $=*, 1 P E 10,3 / 1$

12 FORMAT $(/)$

14 FOFMAT (* NCLC $=*$. I5, * TOTAL NO., AREA =*,1P2E11.4.

$1 *$ AVER.RAD.,AREA,MASS =*,1P3E12.4/1

16 FORMAT I* NUMEER OF PARTICLES IN EACH CLASS*1/3011X,1P 10E13.5/1)

17 FORMATI* PARTICLE RADII*/130(1X,1P10E13.5/1)

18 FORMAT (* TOTAL MASS IN EACH CLASS*/130 $11 X, 1 P 10 E 13.5 / 1)$

21 FORMAT (* COLL.MASS, OIS.SIL. $=*, 2 F 8,4,3 X, *$ SUM $=4, F 8,4,3 X$,

1*SHOJLD $B E=*, F 8,4,3 X, * P E R C E N T$. OIF. $=*, F 8.411$

22 FORMAT (* SALT MOLALITY,SOL, CORR , FACTOR $=*, 1 P E 12,4,0 P F 8,4,3 \mathrm{X}$

1 *SOD. ACT. $=*, 1 P E 13,4,3 X, * P H N, F P H, F I N T=*, O P 3 F B, 4 / 1$

23 FORMAT (* TOT , F,ALPF $=*, 1$ PE10,3, OPF8,4,2X, *RKFL, RKF2 =*, 1P2E 10.3,

$12 X, *$ RATEKF, RATEKT $=*, 1 P 2 E 10.3 / 1$

24 FORMATI* PKW,PKSIL, PKF $=*$, 3F7.3,2X, *GSIL, GF, ONA =*,3F7.4, $2 X$,

1 *EPSD, DENS $=*, F 6.2, F 7,4,2 X, * A D H, B D H=*, 2 F 7,4 / 1$

100 F ORMAT $(/ / 1 X, 26(* \neq F \neq \neq * *) / 1)$

RETURN

END 


\section{List of References}

Abraham, F.F., "Multistate Kinetics in Nonsteady-State Nucleation: A Numerical Solution." J, Chem. Phys., 51, 1632-8 (1969).

Abraham, F. F., Homogeneous Nucleation Theory: The Pretransition Theory of Vapor Condensation. Academic Press (New York, 1974)

Acker, E. G., "The Characterization of Acid-Set Silica Hydrosols, Hydrogels, and Dried Gel", J. Coll. Interface Sc1. 32, 41-54 (1969).

Adamson, A.W. Phystcal Chem1stry of Surfaces, 3rd ed. John Wiley \& Sons (New York, 1976).

Alexander, G. B., "The Polymerization of Monosilicic Acid", J. Phys . Chem. 76, 2094-2096 (1954).

Alexander, G. B., "The Effect of Particle Size on the Solubility of Amorphous Silica in Water", J. Phys: Chem 61, 1563-4:(1957)

Allen, L.H. "Stability of Colloidal Silica," a PhD Thesis in physical chemistry, Clarkson College of Technology, Potsdam, New York. University Microfilms 70-22,583.

Allen, L. H. and E. Matij evic, "Stability of Colloidal Silica. I. Effect of Simple Electrolytes", J. Coll. Interf. Sc1. 31, 287-296 (1969).

Allen, L. H: and E. MatijeviC, "Stability of Colloidal Silica. II. Ion Exchange", J. Co11. Interf. Sc1. 33, 420-429:(1970).

Allen, L. H. and E. MatifeviC, "Stabillty of Colloidal S1lica. III. Effect of Hydrolyzable Cat1ons", J. Col1. Interf. Sc1. 35, 66-75 (1971)

Allen, L. H., E. Matijevit and L. Meites, "Exchange of $\mathrm{Na}^{+}$for the Silanolic Protons of S1l1ca", J. Inorg. Nucl . Chem. 33, 1293-1299 (1971).

Austin, A. L., A. W. Lundberg, L. B. Owen, and G. E. Tardiff, "The LLL Geothermal Energy Program Status Report January 1976 - January 1977", Part 4, Report UCRL-50046-76. Lawrence Livermote Laboratory, Apr11 27, 1977.

Barkman, J. H. and D..H. Davidson, "Measuring Water Quality and Pred1cting Well Impairment", J. Petroleum Tech.,pp. 865-873 (1972).

Baumann, H., "Polymerization and Depolymerization of Silicic Acid under Different Cond1tions", Kol1. Zeltsch. 162, 28-35 (1959) (In German.)

Baumann, H., "The Production of Pure and Perturbed Quartz Surfaces and their Relation under Various Treatments", Fortschr. Kollo1de u. Polymere 55, 37-44 (1971) (In German).

Bellakov, V. N., N. M. Soltivsky1, D. N. Strazhesko and V. V. Strelko, "The Dependence of the Ionization Constant of Silicic Acids Upon their Degree of Polymer1zation", Ukr. KhIm. Zh. 40, 236-7 (1974) (In Russian.) 
Bolt, G.H. "Determination of the Charge Density of Silica Sols." J. Phys. Chem. 61, $1166-9 \cdot(1957)$.

Brunauer, S. D., D. L. Kantro and C. H. We1se, "The Surface Energies of Amorphous Silica and Hydrous Amorphous Silica", Canadian J. Chem. 34, 1483-1496:(1956).

Busey, R. H, and R. E. Mesmer, "The Ionlzation of Water In NaCl Media at $300^{\circ} \mathrm{C}$ ", J. Solution Chem :, 5, 147-152:(1976).

Busey, R. H. and R. E. Mesmer, "Ionization Equilibria of Silicic Acid and Polysilicate Formation in Aqueous Sodium Chloride Solutions to $300^{\circ} \mathrm{C}$ ", In press, 1977.

Butler, J.N., Ionic Equilibria: A Mathematical Approach. Addison-Wesley (Reading, Mass., 1974).

Cherkins'kyi, Yu. S. and I. S. Kn'az'kova, "On the Reactivity of the Complex Silicate Cation in Aqueous Solution", Dokl. Akad. Nauk SSSR, 212, 647-8 (1973). (In Russion).

Collins, F.C., "Time Lag in Spontaneous Nucleation Due to Non-Steady State Effects." Zeitschr. f. Elektrochemie, 59, 404-7(1955).

Cosner, S.R., and J.A. Apps, "A Compilation of Data on Fluids from Geothermal Resources In the Unfted States", Report LBL-5936, Lawrence Berkeley Laboratory, May 1978.

Crerar, D. A. and G. M. Anderson, "Solubllity and Solution Reactions of Quartz in Dilute Hydrothermal Solutions", Chemical Geology, $\underline{8}, 107-122$ (1971).

Cuéllar, G., "Behavior of Silica in Geothermal Waste Waters", Proceedings [of the] Second United Nations Symposium on the Development and Use of Geothermal Resources, San Francisco, California, USA, 20-29 May 2975. pp. 1337-1347.

Dav1s, J. A., R.0. James, and J.0. Leckie, "Surface Ionization an: Complexation at the Oxide/Water Interface: I. Computation of Electrical Double Layer Properties in Simple Electrolytes." J. Coll. Interface Sc1., 63, 480-499 (1978).

Dugger, D. L., J. H. Stanton, B. N. Irby, B. L. McConnel1, W. W. Cummings and R. W. Maatman, "The Exchange of Twenty Metal Ions with the Weakly Acidic Silanol Group of Silica Ge1", J. Phys. Chem. 68, 757-760 (1964).

du Pont Industrial Chemicals Department, "Properties, Uses, Storage and Handling: Ludox Colloidal Silica. Avallable from:

E. I. du Pont de Nemours \& Co. (Inc.)

Industrial Chemicals Department

Wilmington, Delaware 19898

on request at no charge; no date.

Egorov; M. M., V. F. Kiselev, K. G. Krasil'nikov and V. V. Murina, "The Effect of the Nature of the Surface of Silica Gel and Quartz on their Adsorption Properties, III. The Heats of Wetting of Silica by Diverse Liquids" Zhur. Fiz. Khim. 33, 65-73 (1959):(In Russian). 
Egorow, M. M., W. I. Kvlividze, V. F. Kisselev and K. G. Krassilnikow, "The Nature of the Surface and the Adsorption Properties of S1licon Dioxide", Kollo1d-Zeitschr. u. Ze1tschr. f. Polymere 212, 126-138 (1966) (In German.) (Same Egorov as the preceeding, but transliterated into German rather than into English here.)

Einarsson, S. S., A. Vides R, and Gustavo Cuéllar "Disposal of Geothermal Waste Water by Reinfection" Proceedings [of the] Second United Nations Symposium on the Development and Use of Geothermal Resources, San Francisco, Californfa, USA, 20-29 May 1975. pp . 1349-1363.

Eisenberg, D., and W. Kauzmann, The Structure and Properties of Water, Oxford University Press (New York and Oxford, 1969) . Pp - 150-55.

Engelhardt, G., W. Althenburg, D. Hoebbel and W. Wieker, "29 Si NMR Spectroscopy on Silicate Solutions, IV. Investigations of the Condensation of Monosilicic Ac1d", 2. anorg • allg. Chem. 428, 43-52 (1977) (In German).

Engelhardt, G., D. Zelgan, H. Jancke, D. Hoebbel and W. Wieker, "29Si NMR Spectroscopy on Silicate Solutions, II. The Dependence of the Structure of Silicate Anions in Aqueous Solutions of Sodfum Silicates upon the Na:Si

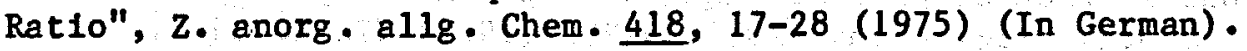

Feder, J., K.C. Russell, J. Lothe, and G.M. Pound, "Homogeneous Nucleation and Growth of Droplets In Vapours." Advances in Physics, 15, 111-178.

Fournier, R. 0. and J.J. Rowe, "The Solub1lity of Amorphous Silica in Water at High Temperatures and Pressures", American Mineralogist, 62, 1052-6 (1977)

Frledberg, K.D., "Factors which Influence the Rate of Hydrolys Is of Condensed Silicic Acid", Beitrage Silikose Forsch. Sonderband, $1,49-56$ (1955) (In German).

Goto, K., "Effect of $\mathrm{pH}$ on Polymerization of Silicic Acid", J. Phys. Chem. 60, $1007-8 \cdot(1956)$.

Greenberg, S.A., "The Depolymerization of silica In Sodium Hydroxide Solutions" J. Phys. Chem. 61, 960-965 (1957).

Kahn, H. H. and H. Stumm, "Kinetics of Coagulation" with Hydrolized AI (III): The Rate Determining Step", J. Co11. Interf. Sc1. 28, 134-144 (1968).

Ha1r, M. L. and W. Hert1, "Acldity of Surface Hydroxyl Groups", J. Phys. Chem. 74, $91-94$ (1970).

Harned, H.S., and B.B. Owen, The Physical Chemistry of Electrolyte Solutions, 3rd Ed. Reinhold (New York, 1958).

Harvey, W. W., M. J. Turner, J. Slaughter and A. C. Makrides "Study of Silica Scaling from Geothermal Brines: Progress Report for Period March, 1976 September 1976", EIC Corporation, 55 Chapel Street, Newton, Mass. 02158, October 1976. Report number C00-2607-3.

Heston, W. M., Jr., R. K. Iler and G. W. Sears, Jr. "The Adsorption of Hydroxyl Ions from Aqueous Solution on the Surface of Amorphous Silica", J. Phys. Chem. 64, 147-150 (1960). 
Hoebbel, D. and W. Wieker, "The Constitution of the Tetramethylammonium Silicate $1.0 \mathrm{~N}\left(\mathrm{CH}_{3}\right)_{4} \mathrm{OH} \cdot 1.0 \mathrm{SiO}_{2} \cdot 8.0-8.3 \mathrm{H}_{2} \mathrm{O}^{\prime \prime}, \mathrm{Z}$. anorg alig. Chem. 384, 43-52 (1971) (In German)

Hoebbel, D., and W. Wieker, "On the Constitution of the Silicate Anion in the Copper Ethylenediamine Silicate of the Composition CuO $2 \mathrm{~S}^{\circ} \mathrm{O}_{2} \cdot 2 \mathrm{en} \cdot 7 \cdot 4 \mathrm{H}_{2} \mathrm{O}^{\prime \prime}$, Z. Chemie 12, 295-7 (1972) (In German).

Hoebbel, D. and W. Wieker, "The Condensation Reactions of Monosilicic Acid", Z. anorg • allg. Chem 400, 148-169 (1973) (In German).

Hoebbel, D. and W. Wieker, "On the Constitution of a Silicate with the Anion

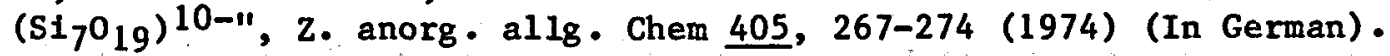

Hoebbel, D., W. Wieker, P. Franke and A. Otto, "On the Constitution of the New Silicate Anton $\left(\mathrm{Si}_{10} \mathrm{O}_{25}\right)^{10-",} \mathrm{Z}$. anorg a allg. Chem. 418, 35-44 (1975). (In German).

Hoebbel, D., W. Wieker, J. I. Smolin, J. F. Sepelev and R. Pomes, "On the Constitution of Silicate Anions in Cobalt Ethylenediamine Silicate", z. anorg - a1.1g - Chem. 423, 225-230 (1976) (In German).

Holt, P. F. and D. T. King, "The Chemistry of the Sllica Surface", J. Chem. Soc. (London), 773-779 (1955).

Howard, J., et al., "Geothermal Resource and Reservior Investigations of U.S. Bureau of Reclamation Leaseholds at East Mesa, Imperial Valley, California", Report LBL-7094, Lawrence Berkeley Laboratory, Berkeley, October 1978.

Huggins, C. M., "Proton Magnetic Resonance Study of the Basicity of the Silicon-0xygen Bond", J. Phys. Chem. 65, 1881-1884 (1961).

Hurd, C. B. and R. W. Barclay, "Studies of Sillclc Acld Gels. X. The Time of Set of Gel Mixtures Containing High Concentrations of Mineral Acids", J. Phys. and Co11. Chem. 44, 847-851 (1940).

Hurd, C. B. and H. A. Letterson, "Studies on Silicic Acid Gels. I. Measurements on Surface Tension during Setting and the Effect of Temperature on the Time of Set", J. Phys. and Co11. Chem., 36, 604 (1932).

Hurd, C. B., C. L. Raymond and P. Schuyler Miller, "Studies of Silicic Acid Gels. IV. The Effect of the Hydrogen Ion Concentration Upon the Time of Set", J. Phys. and Coll. Chem. 38, 663-674 (1934).

Hurd, C. B, and H. E. Scheffer, "Studies of Silicic Acld Gels. XI. The Effect of the Concentration of Silica Upon the Time of Set", J. Phys. and Co11. Chem., 45, 588-594 (1941).

Hurd, C. B. and P. Schuyler Miller, "Studies of Sillcic Acid Gels. II. The Time of Set as a Function of Temperature", J. Phys . and Coll. Chem. 36, 2194-2204 (1932). 
Iler, R. R., "Polymerization of Silicic Acld: Catalytic Effect of Fluoride" J. Phys. Chem. 56, 680-683:(1952).

Iler, R. K., The Colloid Chemistry of Silica and Silicates, Cornell University Press (Ithaca, New York, 1955).

Iler, R. K., "Colloldal Silica", pp. 1-100 in Surface and Colloid Science, Vo1. 6, E. Mat1jevil, ed,, John Wiley \& Sons, Inc. (New York, 1973).

Iler, R. K., "Effect of Adsorbed Alumina on the Solubility of Amorphous Silica In Water", J. Coll. Interface Sc1., 43, 399-408:(1973).

Iler, R. K., Lectures on silica chemistry presented at the Lawrence Livermore Laboratory, October 14-15, 1975.

Iler, R. K., "Coagulation of Colloidal Sillca by Calctum Ions, Mechanism, and

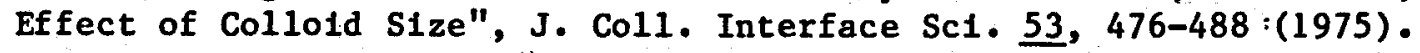

Iler, R. K., "The Effect of Surface Aluminosilicate Ions on the Propertles of Colloidal Silica", J. Coll. Interface Sc1., 55, 25-34:(1976).

Iler, R.K., The Chemistry of Silica: Solubility, Polymerization, Colloid and Surface Properties, and Blochemistry; John Wiley Sons (New York, 1979).

Irvine, T. F., Jr., and J. P. Hartnett. Steam and Air Tables in S. I. Units: Including Data for Other Substances and a Separate Mollier Chart for Steam. Hemisphere Publishing Corporation (Washington, 1976) . Pp.1-16

Johnson, R.A., and J.D. $0^{\prime}$ Rourke, "The Kinetics of Precipitate Formation: Barium Sulfate", J. Phys. Chem., 76, 2124-6:(1954).

Jorgensen, S. S., "Solubility and Dissolution Kinetics of Precipitated Amorphous Silica in $1 \mathrm{M} \mathrm{NaClO}_{4}$ at $25^{\circ} \mathrm{C}$ ", Acta Chem.. Scand. 22 , 335-341 (1968).

Kharaka, Y. K., and I. Barnes, "SOLMNEQ: Solution-Mineral Equilibrium Computations". U.S. Geological Survey Computer Contribution PB-215 899 . Menlo Park, CA., February 1973.

Kubota, K., and K. Aosak1 "ReInjection of Geothermal Hot Water at the Otake Geothermal Field", Proceedings [of the] Second United Nations Sympostum on the Development and Use of Geothermal Resources, San Francisco, CA. USA, 20-29 May 1975. Pp - 1379-1383.

Kv lividze, V. I., "Nuclear Magnetic Resonance at $93^{\circ} \mathrm{K}$ of the Protons of Water Adsorbed on S111ca Ge1", Dok1. Akad. Nauk SSSR 157, 158-161:(1964) (In Russian; also available In English translation).

Loeb, A. L., J. Th. G. Overbeek and P. H. Wersema, The Electrical Double Layer Around a Spherical Collold Particle: Computation of the Potential, Charge Density, and Free Energy of the Electrical Double Layer Around a Spherical Collolid Particle, M.I.T. Press (Cambridge, Mass., 1961). 
MacKenzie, F.T., and R. Gees, "Quartz: Synthesis at Earth Surface Conditions", Science, 173, 533-5 (1971).

Makrides, A. C., M. J. Turner, W. W. Harvey, J. Slaughter, S. B. Brummer, P. $0^{\circ}$ D. Offenhartz and G. F. Pearson, "Study of Silica Scaling from Geothermal Brines: Final Report for Perlod November 15,"1974 - Apri1 30, 1977", Report C00-2607-5. EIC Corporation, 55 Chapel Street, Newton, Massachusetts 02158, January 1978.

Marsh, A. R. III. G. KleIn and T. Vermeulen, "Polymerization Kinetics and Equilibria of Silicic Acid in Aqueous Syśtems", Report LBL-4415, Lawrence Berkeley Laboratory, October 1975.

Matijevic, E., "Colloid Stability and Complex Chemistry", J. Coll. Interface Sc1. $43,217-245$ (1973).

Messer, P. H., D. S. Pye and J. P. Gallus, "Injectivity Restoration of a Hot Brine Geothermal Injection Well", Soclety of Petroleum Eng Ineers Paper $6761 \cdot(1977)$.

Midkiff, W. S. and H. P. Foyt, "Amorphous Silica Scale in Cooling Waters", Submitted to the Cooling Tower Institute, Annual Meeting, Houston, Texas January 19-21, 1976, Report LA-UR-75-2313. Los Alamos Scientific Laboratory.

Midkiff, W. S. and H. P. Foyt, "Silica Scale Technology and Water Conservation". Submitted to the meeting of the National Association of Corrosion Engineers, March 14-18, 1977, San Francisco, CA., Report LA-UR-76-2500. Los Alamos Scientific Laboratory.

Mozzi, R. L. and B. E. Warren, "The Structure of Vitreous S1lica", J. App1. Cryst. 2, 164-172 (1969).

Naumov, A.B., B.N. Ryzhenko, and I.L. Khodakov ski1, Handbook of Thermodynamic Data:(Spravochnik Termodinamicheskikh Velichin), Moscow, Atomizdat (1971).

Netherton, R., and L. B. Owen, "Apparatus for the Field Evaluation of Geothermal Effluent Injection", Geothermal Energy - Novelty Becomes Resource: Transactions [of the] Geothermal Resources Council Annual Meeting, Hilo, Hawa1i, 25-27 July, 1978. Vo1. II, PP. 487-490.

$0^{\circ}$ Connor, T. L. and S. A. Greenberg, "The Kinetics of the Solution of Silica In Aqueous Solutions", J. Phys. Chem. 62, 1195-1198:(1958).

Owen, L. B., P. W. Kasameyer, R. Netherton, and L. Thorson "Predicting the Rate by which Suspended Solids Plug Geothermal Injection Wells." Proceedings [of the] Third Workshop [on] Geothermal Reservoir Englneering, held at Standord University, December 14-16, 1977. Report SGP-TR-25. Pp.163-171.

Ozawa, T. and Y. Fuj 11, "A Phenomenon of Scaling In Production Wells and the Geothermal Power Plant in the Matsukawa Area", Geothermics, Sp. Is sue 2, Vol. 2, part 2, 1613-1618:(1970). 
Penner, S. S., "New Method for Determination of the Activation Energy for the Gelation of Silicic Acid Solutions", J. Polymer Sc1. 1, 441-444:(1946).

PhIllips, S. L., A. K. Mathur, and R. E. Doebler "A Study of Brine Treatment" Report EPRI-476 and LBL-6371. Lawrence Berkeley Laboratory, November 1977.

Pitzer, K. S., "Thermodynamics of Electrolytes. I. Theoretical Basis and General Equations", J. Phys.. Chem. 77, 268-277:(1973).

Piwinsk11, A. J., and R. Netherton, "An Experimental Investigation of the Permeability of Kayenta and St. Peter Sandstones to Hypersaline Brine In the Temperature Interval 70 to $90^{\circ} \mathrm{C}$ at $10.3-\mathrm{MPa}$ Confining Pressure", Report UCRL-52382. Lawrence Livermore Laboratory, December 22, 1977.

Quong, R., F. Schoepfiln, N. D. Stout, G. E. Tardiff and F. R. McLain (1978) "Processing of Geothermal Brine Effluents for Injection", Geothermal Energy - Novelty Becomes Resource: Transactions [of the] Geothermal Resources Council Annual Meeting, Hilo, Hawai1, 25-27 July, 1978, Vol. II, pp. 551-554.

Reardon, E.J., "Complexing of Silica by Iron(III) In Natural Waters", Chemical Geology, 25, 339-345 (1979).

Rimstidt, J.D., "The Kinet1cs of S11ica-Water Reactions", Thesis, The Pennsylvania State University, The Graduate School, Department of Geosciences, May 1979.

Rothbaum, H. P. and B. H. Anderton, "Removal of Silica and Arsenic from Geothermal Discharge Waters by Preclpitation of Useful Calclum Silicates", Proceedings [of the] Second United Nations Symposium on the Development and Use of Geothermal Resources, San Francisco, California, USA, May 2029, 1975, Pp. 1417-1425.

Rothbaum, H.P., B.H. Anderton, R.F. Harrison, A.G. Rohde and A. Slatter, "Effect of Silica Polymerisation and pH on Geothermal Scaling", Geothermics, $\underline{8}, 1-20$ (1979).

Rothbaum, H.P., and A.G. Rohde, "Kinetics of Sillca Polymerization and Deposition from Dilute Solutions Between $5^{\circ} \mathrm{C}$ and $180^{\circ} \mathrm{C} "$, (In press, 1979).

Russe11, K. C., "LInked Flux Analysis of Nucleation In Condensed Phases", Acta Met. $16,761-769:(1968)$.

Russe11, K. C., "Gra1n Boundary Nucleation Kinet1cs", Acta Met, 17, 1123-1131 $\cdot(1969)$.

Santsch1, P. H. and P. W. Schindler, "Complex Formation in the Ternary

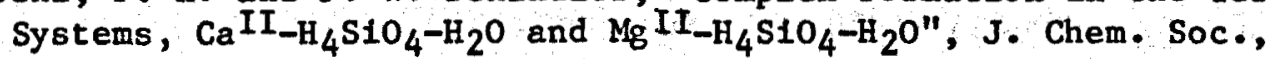
Dalton Trans., pp. 181-184:(1974). 
Schindler, P. W., B. Furst, R. Dick and P. U. Wolf, "Ligand Properties of Surface Silanol Groups, I. Surface Complex Formation with $\mathrm{Fe}^{3+}, \mathrm{Cu}^{2+}$, $\mathrm{Cd}^{2+}$ and $\mathrm{Pb}^{2+"}, \mathrm{~J}$. Coll. Interface Sc1. 55, 469-475:(1976).

Schindler, P. and H. R. Kamber, "The Acidity of Silanol Groups", Helv. Chim. Acta $\underline{51}, 1781-1786:(1968)$ (In German).

Sears, G. W., Jr., "Determination of Specific Surface Area of Colloidal Silica by Titration with Sodium Hydroxide", Anal. Chem., 28, 1981:(1956).

Siever, R., "Sillca Solubility, $0^{\circ}-200^{\circ} \mathrm{C}$. and the Diagenesis of Siliceous Sediments", J. Geology, 70, 127-150:(1962).

S1llén, I. G. and A. E. Marte11, Stab1lity Constants of Metal-Ion Complexes, London: The Chemical Society, Burlington House, W. I. 1964

Silvester, L. F. and K. S. Pitzer, "Thermodynamics of Geothermal Brines. I. Thermodynamic Properties of Vapor-Saturated $\mathrm{NaCl}$ (aq) Solutions from $0-300^{\circ} \mathrm{C}$. Report LBL-4456, Lawrence Berkeley Laboratory, January 1976.

Smolin, Yu. A., "The Crystal Structure of $\left[\mathrm{N1}\left(\mathrm{NH}_{2} \mathrm{CH}_{2} \mathrm{CH}_{2} \mathrm{HN}_{2}\right)_{3}\right]_{3} \cdot \mathrm{Si}_{6} \mathrm{O}_{15}$ $26 \mathrm{H}_{2} \mathrm{O}$; a New Type of Silicon-0xygen Double Ring, $\mathrm{Si}_{5} \mathrm{O}_{15} 6^{-1}$, Chem. Comm., p. $395 \cdot(1969)$.

Smolin, Yu.I., Yu.F. Shepelev and I. K. Butikova, "Crystal Structure of $4\left[\mathrm{Cu}\left(\mathrm{NH}_{2} \mathrm{CH}_{2} \mathrm{CH}_{2} \mathrm{NH}_{2}\right)_{2}\right] \cdot \mathrm{Si}_{8} \mathrm{O}_{20} \cdot 38 \mathrm{H}_{2} \mathrm{O}^{\prime \prime}$, Sov. Phys. Crystallogr. 17, $10-15 \cdot(1972)$ (In English).

Smolln, Yu I., Yu F.Shepelev, R. Pomes, D. Khobbel" and V. Viker, "The Silicon-oxygen radical [ $\left[\mathrm{Si}_{8} \mathrm{O}_{18}(\mathrm{OH})_{2}\right]$ in the $2\left[\mathrm{Co}\left(\mathrm{NH}_{2} \mathrm{HC}_{2} \mathrm{CH}_{2} \mathrm{NH}_{2}\right)_{3}\right]$ $\mathrm{Si}_{8} \mathrm{O}_{18}(\mathrm{OH})_{2} \cdot 16.4 \mathrm{H}_{2} \mathrm{O}$ Crystal", Sov. Phys . Crystallogr., 20, 567-571 $\cdot(1976)$ :(In Eng 11sh).

Sommer, L. H., Stereochemistry, Mechanism and Silicon: An Introduction to the Dynamic Stereochemistry and Reaction Mechanisms of Silicon Centers, McGraw-H111 (New York; 1965).

Stade, H. and W. Wieker, "The Rinetics of Decomposition of Polysillcic Acid $\left(\mathrm{H}_{2} \mathrm{SiO}_{3}\right)_{x}$ in Aqueous Solution", $\mathrm{Z}$. anorg - allg. Chem. 384, 53-66 $\cdot(1971)$ (In German).

Stöber, W., "Formation of Silicic Acid in Aqueous Suspensions of Different Silica Modifications", pp. 161-182 in Advances in Chemistry Series 67, Am. Chem. Soc., 1967.

Strazhesko, D. N., V. B. Strelko, V. N. Belyakov and S. Rubantk, "Mechanism of Cation Exchange on Silica Ge1s", J. Chromatography 102, 191-195 (1974).

Strelko, V. V., "The Mechanism of Polymerization of Silicic Acids", Koll. Zhur. 32, 430-436:(1970) :(In Russian).

Tai An-pang and Chen Yung-san, "Studies of Silicic Acid and Its Salts: Ef fect of Fluoride Ions on Polymerization of Silicic Acid", Scientia Sinica, 14, 73-79 (1965). 
TeZak, B., E. Matif evic and K. Schulz, "Coagulation of Hydrophob1c Sols in Statu Nascend1. I. Determination of Coagulation Values", J. Phys. Collold Chem. 55, 1557-1567:(1951).

Thorhallsson, S., K. Ragnars, S. Arnorsson and H. Kristmannsdottir, "Rapid Scaling of silica in Two District Heating Systems", Proceedings [of the] Second United Nations Symposium on the Development and Use of Geothermal Resources, San Francisco, California, USA, May 20-29, 1975, pp - 1445-1449.

Truesde11, A. H., and B. F. Jones, "WATEQ, a Computer Program for Calculating Chemical Equilibria of Natural Waters." J. Res. U.S. Geol. Soc., 2 , 233-248:(1974).

van Lier, J. A., P. L. de Bruyn and J. Th. G. Overbeek, "The Solubility of Quartz", J. Phys. Chem. 64, 1675-1682:(1960).

Verwey, E. J. W. and J. Th. G. Overbeek, Theory of the Stability of Lyophobic Colloids: The Interaction of Sol Particles Having an Electric Double Layer, Elsevier (Amsterdam-New York, 1948).

Weast, R. C., and S. M. Selby, Handbook of Chemistry and Physics: A ReadyReference Book of Chemical and Physical Data, 48th Ed. The Chemical Rubber Company (Cleveland, 1967).

Weres, 0. and S. A. Rice, "A New Model of Llquid Water", J. Am. Chem. Soc., 94, $8983-9002:(1972)$.

Weres, 0., A. Yee and L. Tsao, "Predicting the Precipitation of Amorphous Silica from Geothermal Brines". Proceedings [of the] Fourth Workshop [on] Geothermal Reservolr Eng ineering, held at Stanford University, December 13-15, 1978. Report SGP-TR-30. Pp. 294-299.

Weres, 0., A. Yee and L. Tsao, "Kinetic Equations and Empirical Type Curves for Predicting the Precipitation of Amorphous Silica from Geothermal Brines", Proceedings of the Symposium on 01lfield and Geothermal Chemistry held in Houston, January 22-24, 1979, Paper SPE 7888.

Weres, 0., L. Tsao and E. Iglesias, "The Chemistry of Silica In Cerro Prieto Brines". Report LBL-10166. Lawrence Berkeley Laboratory, April 1980.

West, R. and R. H. Baney, "Hydrogen Bonding Studies. II. The Acidity and Baslcity of Stlanols Compared to Alcohols", J. Am. Chem. Soc. 81, 6145-6148:(1959).

Weston, R. E., Jr. and H. A. Schwarz, Chemical Kinetics, Prentice-Hall, Inc. (Englewood Cliffs, N. J., 1972).

Wiese, G. R., R. O. James, D. E. Yates and T. W. Healy, "Electrochemistry of the Collo1d-Water Interface", pp. 53-102 In J. $0^{\circ} \mathrm{M}$. Bockr1s, ed., International Review of Sclence; Physical Chemistry, Series Two; Volume 6, Electrochemistry, Butterworths (London-Boston, 1976). 
Wirth, G.S. and J.M. Gieskes. "The Initlal Kinetics of the Dissolution of Vitreous Silica in Aqueous Media." (Submitted for publication, 1979.)

Wohlberg, C. and J. R. Buchholz, "Silica in Water in Relation to Cooling Tower Operation", Report LA-5301-MS. Los Alamos Sclentific Laboratory, October 1973.

Yanagase, T., Y. Sug Inohara and K. Yanagase, "The Properties of Scales and Methods to Prevent Them", Geothermics, Sp. Issue 2, Vol. 2, Part 2, $1619-1623:(1970)$.

Yates, D. E., S. Levine and T. W. Healy, "S1te-binding Model of the Electrical Double Layer at the Oxide/Water Interface", J. Chem. Soc., Faraday Transactions I, 70, 1807-1818:(1974).

Young, G. J., "Interaction of Water Vapor with Sillca Surfaces", J. Colloid Sc1. $13,67-85:(1958)$. 